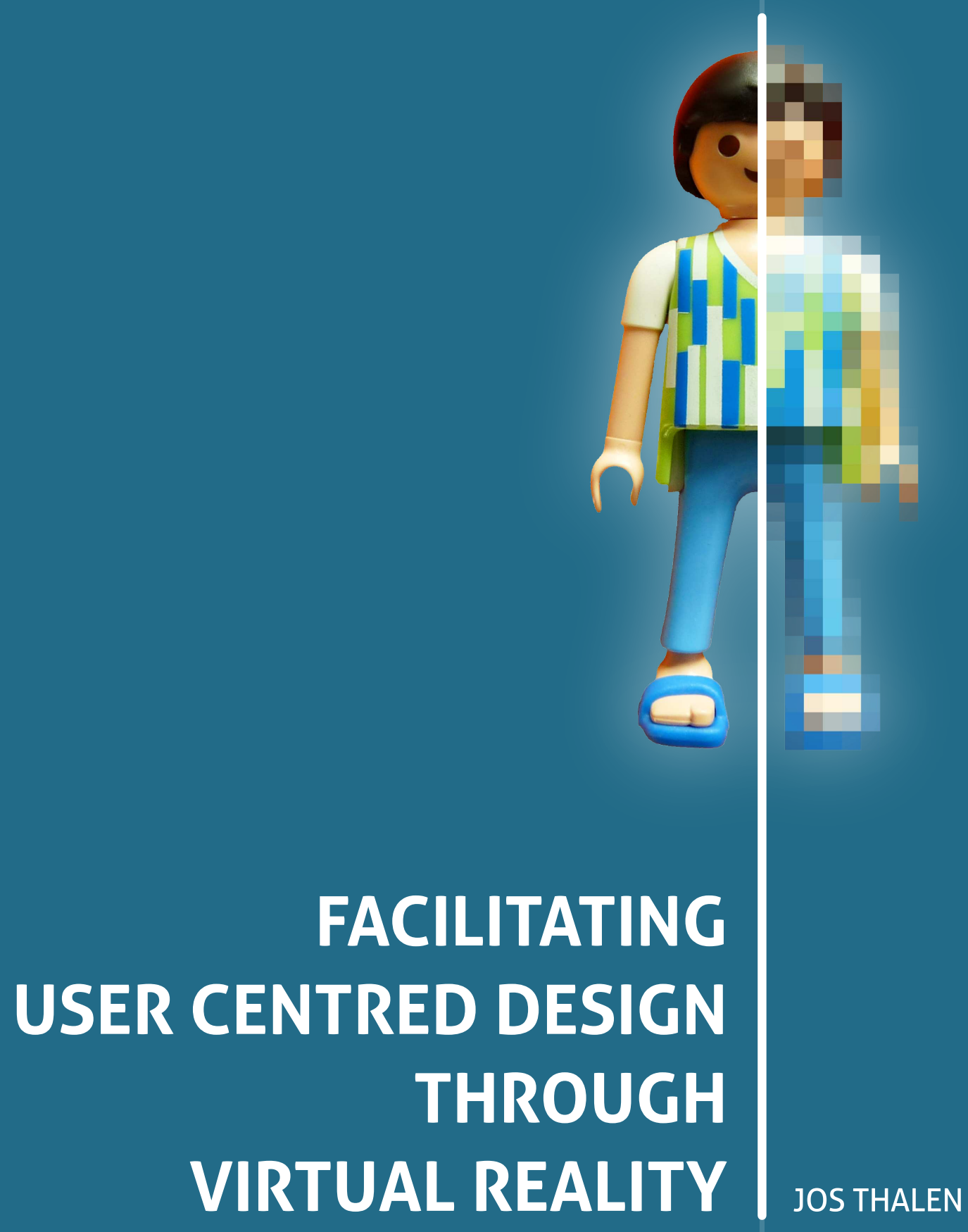




\section{FACILITATING USER CENTRED DESIGN THROUGH VIRTUAL REALITY}

\section{PROEFSCHRIFT}

ter verkrijging van

de graad van doctor aan de Universiteit Twente,

op gezag van de rector magnificus,

prof. dr. H. Brinksma,

volgens besluit van het College voor Promoties

in het openbaar te verdedigen

op woensdag 18 september 2013 om 14.45 uur

door

Joseph Paul Thalen

geboren op 22 februari 1984

te Hoogezand-Sappemeer 
Dit proefschrift is goedgekeurd door:

Prof. dr. ir. F.J.A.M. van Houten (promotor)

Dr. ir. M.C. van der Voort (assistent-promotor) 
Ik geloof dat dit een thesis 
Members of the dissertation committee:

Prof. dr. G.P.M.R. DeWulf

Prof. dr. ir. F.J.A.M. van Houten

Dr. ir. M.C. van der Voort

Prof. dr. K.P.T. Kuutti

Dr. H. Patel

Prof. ir. D.J.van Eijk

Prof. dr. ir. J.B.O.S. Martens

Prof. dr. ir. H. van der Kooij

Prof. dr. V. Evers
University of Twente

University of Twente

University of Twente

University of Oulu

University of Nottingham

Delft University of Technology

Eindhoven University of Technology

University of Twente

University of Twente

The author gratefully acknowledges the support of the Innovation-Oriented Research Programme 'Integral Product Creation and Realization (IOP IPCR)' of the Netherlands Ministry of Economic Affairs, Agriculture and Innovation.

ISBN: 978-90-365-0607-6

DOI: $10.3990 / 1.9789036506076$

Typeset with $\mathbb{A}^{\mathrm{T}} \mathrm{E} \mathrm{X}$, printed by Gildeprint Drukkerijen, The Netherlands

Copyright (C) Jos Thalen, 2013

All rights reserved. No part of this publication may be reproduced, stored in a retrieval system or transmitted, in any form or by any means, electronic, mechanical, photocopying, recording or otherwise, without the prior written permission of the author. 


\section{Preface}

Believe it or not, but I never expected to finish this thesis. This is mainly because I never planned to start writing one in the first place. It was only after about one year into the research project when I first started to seriously consider a scientific career. I think this illustrates the overall lack of structure in my professional career and it is probably the only way to explain how I first pursued a career as a fighter pilot, was then trained as an industrial designer, became a PhD student and ended up as an entrepreneur. Anyway, three and a half years ago, the REPAR project offered exactly the things I was looking for: a practical setting provided by industrial partners, a focus on user centred design and an opportunity to explore new technologies. Surprisingly, the project lived up to all my expectations and I am quite content with the results. Obviously this would not have been the case without the help of a lot of people, including you, probably.

First of all I would like to thank my promotor Fred van Houten for giving me the freedom to do whatever I wanted, as long as it would result in something good. The research would have ended without a thesis if it was not for the support of my assistant-promotor Mascha van der Voort. Thank you for helping me turn three years of case studies, workshops and other practical experiences into a PhD thesis and a company.

Javier and Derya, it has been a pleasure working with you in the REPAR project. I particularly enjoyed the time we spent being embedded in the design departments of the industrial partners involved in the project. It was fun to see our professional and cultural backgrounds mix (and sometimes clash), especially during lunch, and everyday after 14:00, when Spanish people apparently fall asleep automatically. Even though our sub-projects eventually followed their own paths, I think we managed to end up with quite a successful project. On a related note I would also like to thank Jacques Terken for leading the project and for organising a successful final symposium, and Jean-Bernard Martens for participating in progress meetings and for critically reviewing our work.

When reading this thesis you will notice that about $70 \%$ of the research involved direct collaboration with industrial partners. I am very grateful to all three companies for their openness and willingness to cooperate. It allowed me to conduct numerous experimental workshops, to test prototype applications in practice and to conduct evaluations based on real-life design cases. Because of confidentiality reasons the names of the companies are not included in the thesis. Nevertheless I would like to thank Eddy, Ron, Abbie, Estella and Guido from 'company A', Freek, Martijn, Roderick, Alex and Rinse from 'company B', and Roland, Frits, Jeroen, Jan and Rutger from 'company $C$ '. Please pass the word to your colleagues who were involved in the workshops and test sessions. 
The other $30 \%$ of the research took place in the department of Design, Production and Management at the University of Twente. It took about a month to get used to the people, traditions and the working habits of the group. I'm afraid it will take much longer to get used to not working there any more. The drawback of a big group is that you can not thank everyone personally, so I will just say thanks to the entire 'Koffietafel' for all the talks, laughs, moments of silence and barbecues. Of course there are also some colleagues who l'd like to mention separately. First of all, Roy Damgrave and Fjodor van Slooten, thank you for the technical support in the VR lab. I would also like to thank Mieke, Irene, Arie Paul, Frederick, Frederik, Mascha, Jeroen and Julia for the meetings, discussions and social events we had in the Use Anticipation in Product Design group. Julia, I enjoyed working together for the MST case and several other VR demos and papers. Last but not least, Jesse, thank you for your valuable contribution to the virtual persona case study.

And then some words about N-211. I think the reputation of this room is best illustrated by a brief review of literature. In 2011, de Boer (2011) claimed that the "absurd office humour and practical jokes" had a positive effect on the quality of life at the university. Interestingly, these practical jokes are described somewhat more reserved as "entertaining actions" by Hoolhorst (2012), one of the more flamboyant inhabitants of N-211. In the same year ten Dam (2012) aptly characterised the room as a "small but crazy community within the OPM community". Hopefully these examples explain why I am proud as well as grateful to have been part of N-211 for more than three and a half years.

At last, I would like to thank all the fencers and mountain bikers who pulled me away from work every now and then. The weekly fencing training and the regular cycling weekends helped to keep the mind and body balanced.

Now that I have thanked everyone who somehow contributed to my research or the context in which it took place, I will spend the last paragraph on saying sorry to those I completely neglected over the last couple of years. Lieve familie, geloof het of niet, maar dit boekje is dus waar ik de afgelopen jaren naar toegewerkt heb. Nee, ik was al afgestudeerd, dit is meer onderzoek dan studie. En ja, ik werd hier voor betaald. Nee, ik ben nu geen professor. Maar serieus, bedankt voor het onvoorwaardelijk tevreden zijn met wat ik doe zolang ik er maar blij mee ben. Tot slot Marjan; je bent uniek, bijzonder en belangrijk, en niet alleen omdat je de enige persoon ter wereld bent die het proefschrift van $A$ tot $Z$ tot op de letter heeft gelezen (en verbeterd). Ik denk niet dat we het ooit eens zullen worden of mijn vakgebied nou echt wetenschap genoemd mag worden, maar ik dank je wel voor alle ruimte die ik kreeg om er aan te werken. 


\section{Summary}

Most designers are familiar with various forms of concept representations, such as sketches, storyboards or physical prototypes. These representations can facilitate communication with end-users. Involving end-users in the design process, also referred to as User Centred Design (UCD), allows designers to ask what end-users think of a product concept, see how end-users would use a product concept or even ask end-users to assist in the definition of a product concept. Especially in the early stages of the design process, user involvement can reduce the risk of expensive and time consuming redesigns in later stages of the design process. However, when developing new, complex or interactive products, traditional concept representations sometimes fail to fully convey the product, interactions or use context. For example, you cannot expect an end-user to provide reliable feedback on the usability of a new mobile phone based on a simple sketch. Not only does the sketch leave out details and interactions, it might also lack cues about the intended use context. Presenting a product concept in a concrete use context or use situation makes it easier for end-users and other stakeholders to understand.

The current research proposes to use Virtual Reality (VR) technologies to create realistic representations of future products, user-product interactions and use contexts. VR technologies create an alternative reality in which worlds, objects and characters can be experienced that may not yet be available in reality. By deploying these technologies in the early stages of a UCD process, VR can:

- Provide an interactive and realistic confrontation with future use situations

- Make complex situations and information accessible to all stakeholders

- Support early stage concept generation, presentation and evaluation

Together these opportunities help elicit more profound insights and feedback from end-users in the early stages of the design process, and consequently contribute to creating products that suit end-user needs and expectations better.

In practice, however, VR applications are only relevant if one is able to implement them through an effort that is proportional to the benefits one gets in return. Especially for VR techniques, which are traditionally considered complex, expensive and time consuming to deploy, this is a relevant aspect for the research to investigate. Therefore, in addition to investigating how VR can facilitate early stage UCD activities, the research presented in this thesis looks into whether these applications can be realised by design practitioners. These objectives are summarised as follows:

1. Identify advantageous applications of VR in the early stages of a UCD process

2. Determine the boundary conditions for designers to realise these VR applications themselves 
Both objectives were first addressed in a specific design context by conducting three industrial case studies. In each case study a VR application that facilitates early stage UCD activities was identified, developed and deployed in practice. By evaluating the case study results across various design contexts, insights were gained into the effectiveness of VR applications in different design domains, as well as the boundary conditions that different designers have with respect to the realisation of these applications.

This led to the following conclusions:

1. In the early stages of the product design process, VR facilitates UCD activities by providing an interactive and integral virtual representation of future use situations in which product concepts can be generated, presented or evaluated.

2. Within this scope of applications, low-end off the shelf VR techniques and low fidelity models provide sufficient means for realising these virtual representations.

3. The primary challenge for designers to deploy such applications lies in

(a) the identification of an effective VR application and

(b) the selection of appropriate means to realise this application.

Based on these conclusions, the objectives of this research have been achieved; 1) It was shown that VR can effectively facilitate UCD activities by providing a virtual representation of future use situations, and 2) these applications can be realised by designers themselves, once they have been provided with appropriate preparation and execution tools. Supporting techniques for the selection of appropriate preparation and execution tools, consisting of an exploration workshop and a set of practical selection guidelines, have been developed, deployed and evaluated within this research. These techniques can be used by the designers themselves, or by an external consulting party. 


\section{Contents}

Preface $\quad$ i

Summary iii

1 Introduction $\quad \mathbf{1}$

1.1 User Centred Design \& Virtual Reality . . . . . . . . . . . . . . . . . . . . . . 1

1.2 Problem description . . . . . . . . . . . . . . . . . . . . . 2

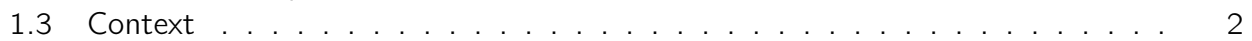

1.3.1 Practical context . . . . . . . . . . . . . . . . 4

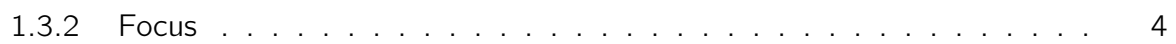

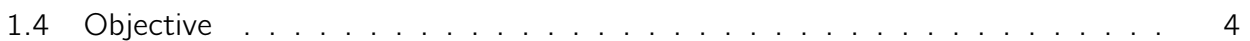

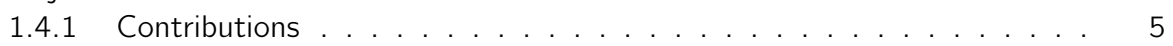

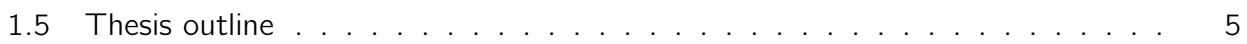

2 Background $\quad 7$

2.1 User Centred Design . . . . . . . . . . . . . . . . . . . . . . . . . . . . . . . 7

2.1.1 The product development process . . . . . . . . . . . . 8

2.1.2 User involvement in the product development process . . . . . . . . . . 9

2.1.3 Characteristics of UCD activities . . . . . . . . . . . . . . . . . 11

2.2 Virtual Reality . . . . . . . . . . . . . . . . . . . . . . . . . . . 12

2.2.1 Definition \& overview . . . . . . . . . . . . . . . . . . . . . 12

2.2.2 Applications in product development . . . . . . . . . . . . . . . . 14

2.2 .3 Trends \& opportunities . . . . . . . . . . . . . . . . . . . . . . . 16

2.2 .4 Discussion . . . . . . . . . . . . . . . . . . . . . . 16

2.3 Field study . . . . . . . . . . . . . . . . . . . . . . . . . . . . . . . . . . . . . . . 17

2.3 .1 Interviews \& site visits . . . . . . . . . . . . . . . . . . . . . 17

2.3.2 VR demonstration session . . . . . . . . . . . . . . . . . . . . 18

2.3 .3 Conclusion . . . . . . . . . . . . . . . . . . . . . . . 20

2.4 Discussion . . . . . . . . . . . . . . . . . . . . . . . . . . . . . . . . . . 20

2.4.1 Research objective . . . . . . . . . . . . . . . . . . . 21

2.4 .2 Towards an approach . . . . . . . . . . . . . . . . . . . . 22

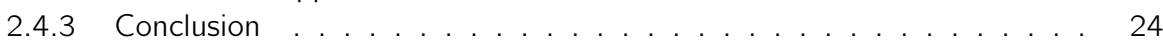


3 Approach 25

3.1 Overview ............................... 25

3.1.1 Key characteristics ...................... . 25

3.1 .2 Structure . . . . . . . . . . . . . . . . . . . . . . 26

3.1 .3 Output ........................... . . 28

3.2 Case study method .. . . . . . . . . . . . . . . . . . . . . . . . . . 29

3.2.1 Explorative workshop . . . . . . . . . . . . . . . . . . . . . . . . 29

3.2.2 Prototype development . . . . . . . . . . . . . . . . . . . . . . . . . 31

3.2.3 Application validation ........................ 31

3.2 .4 Tool selection . . . . . . . . . . . . . . . . . . . . . . . 32

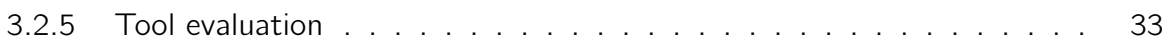

3.3 Generalisation method . . . . . . . . . . . . . . . . . . . . . . . . 33

3.3.1 Session Structure . . . . . . . . . . . . . . . . . . . . . . . . . . 34

3.3 .2 Output ........................... 34

3.4 Implementation ............................. . . . . 35

4 Case Study $1 \quad 37$

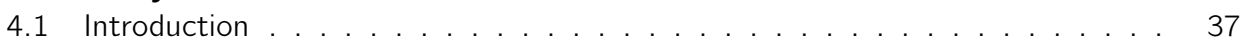

4.2 Exploration .......................... 38

4.2.1 Kick-off . . . . . . . . . . . . . . . . . . . . . . . 38

4.2.2 VR exploration workshop . . . . . . . . . . . . . . . . . . . 39

4.2.3 Application description . . . . . . . . . . . . . . . . . . . . . . 42

4.3 Development .. . . . . . . . . . . . . . . . . . . . . . . . . . . . 44

4.3.1 Application prototype . . . . . . . . . . . . . . . . . . 45

4.3.2 Application validation . . . . . . . . . . . . . . . . . 46

4.4 Deployment . . . . . . . . . . . . . . . . . . . . . . . . . . . . . . . 50

4.4.1 Tool selection . . . . . . . . . . . . . . . . . . . . . . . 50

4.4 .2 Tool evaluation ....................... . . 52

4.5 Conclusion .............................. . . . . 53

5 Case Study $2 \quad 55$

5.1 Introduction .............................. 55

5.2 Exploration ............................ . . 56

5.2.1 Kick-off . . . . . . . . . . . . . . . . . . . . . . 56

5.2.2 VR exploration workshop ..................... . 56

5.2.3 Application refinement . . . . . . . . . . . . . . . . . . . . 58

5.2.4 Application definition . . . . . . . . . . . . . . . . . . . . 60

5.3 Development .............................. . 61

5.3.1 Application prototype........................ . 61

5.3.2 Application validation . . . . . . . . . . . . . . . . . . . . 63

5.4 Deployment .. . . . . . . . . . . . . . . . . . . . . . . . . . 68

5.4.1 Tool selection . . . . . . . . . . . . . . . . . . . . . . 68

5.4.2 Tool evaluation ......................... . . . 71

5.5 Conclusion ............................ 73 
6 Case Study $3 \quad 75$

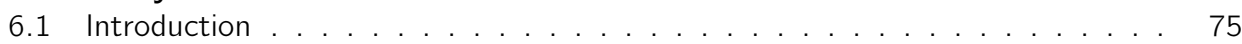

6.2 Exploration . . . . . . . . . . . . . . . . . . . . . 76

6.2 .1 Kick-off . . . . . . . . . . . . . . . . . . . . 76

6.2 .2 VR exploration workshop . . . . . . . . . . . . . . 76

6.2 .3 Application description . . . . . . . . . . . . . . . . . . . . 80

6.3 Development . . . . . . . . . . . . . . . . . . . . . . . . . . 81

6.3.1 Application prototype . . . . . . . . . . . . . . . . . . 82

6.3.2 Application validation . . . . . . . . . . . . . . . . . 83

6.4 Deployment . . . . . . . . . . . . . . . . . . . . . . . . . . 87

6.4 .1 Tool selection . . . . . . . . . . . . . . . . . . . . . . . . . . 88

6.4 .2 Tool evaluation . . . . . . . . . . . . . . . . . . . . . . . . 89

6.5 Conclusion . . . . . . . . . . . . . . . . . . . . . . . . . . 90

7 Generalisation $\quad 93$

7.1 Cross company evaluations . . . . . . . . . . . . . . . . . . . . . . 93

7.1.1 CCE 1: The Virtual Printshop . . . . . . . . . . . . . . . . . . . . . . 94

7.1 .2 CCE 2: Virtual Personas . . . . . . . . . . . . . . . . . 95

7.1 .3 CCE 3: Virtual Annotation . . . . . . . . . . . . . . . . . . 96

7.2 Analysis . . . . . . . . . . . . . . . . . . . . . . . . . . . . . 98

7.2.1 Translation strategies . . . . . . . . . . . . . . . . . . . 999

7.2 .2 Interpretation of results . . . . . . . . . . . . . . . . . 100

7.2.3 Generalised model . . . . . . . . . . . . . . . . . . . . . . . . 101

7.3 Approach for realisation . . . . . . . . . . . . . . . . . . . 105

7.3.1 Application definition . . . . . . . . . . . . . . . 106

7.3 .2 Tool selection . . . . . . . . . . . . . . . . . . . . 106

8 Reflection $\quad 109$

8.1 Research approach . . . . . . . . . . . . . . . . . . . . . . 109

8.1 .1 Structure . . . . . . . . . . . . . . . . . . . 109

8.1.2 Designer centred research . . . . . . . . . . . . . . . . 110

8.1 .3 Gathering data . . . . . . . . . . . . . . . . . . . . . . . . . . . . . . . . . . 112

8.2 Implementation of methods . . . . . . . . . . . . . . . . . . . . . 113

8.2.1 VR exploration workshop . . . . . . . . . . . . . . . . 114

8.2.2 Prototype development . . . . . . . . . . . . . . . . . . . . . 115

8.2.3 Application validation sessions . . . . . . . . . . . . . . . . . 116

8.2 .4 Cross company evaluation sessions . . . . . . . . . . . . . . . 117

8.3 Conclusion . . . . . . . . . . . . . . . . . . . . . . . . . 118

9 Conclusion $\quad 121$

9.1 Facilitating UCD through VR . . . . . . . . . . . . . . . . . . . . . . . 121

9.2 Realisation of VR applications . . . . . . . . . . . . . . . . . . . . 122

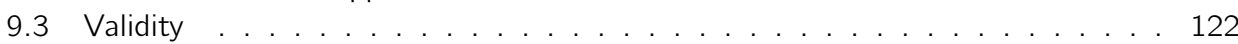

9.3.1 Industrial contexts . . . . . . . . . . . . . . . . . . . . 123

9.3.2 Position in product design process . . . . . . . . . . . . . . . . . . . 123

9.3.3 Range of technologies . . . . . . . . . . . . . . . . . . . 123

9.4 Final thoughts \& future work . . . . . . . . . . . . . . . . . . . . . . . . . . . . 124

9.4 .1 Future work . . . . . . . . . . . . . . . . . . . . . . 124 
A Case Study 1 Data 135

A.1 Application validation . . . . . . . . . . . . . . . . . . . . . . . 136

A.1.1 Application validation form . . . . . . . . . . . . . . . . . . 137

A.1.2 Application validation results . . . . . . . . . . . . . . . 138

A.2 Tool evaluation . . . . . . . . . . . . . . . . . . . . . . . . . . . . . 143

A.2.1 Tool evaluation form . . . . . . . . . . . . . . . . . . . . . . . . 143

A.2.2 Tool evaluation results . . . . . . . . . . . . . . . . . . . . . . 144

A.3 Cross-company evaluation $1 \ldots \ldots \ldots \ldots$

A.3.1 CCE1 forms . . . . . . . . . . . . . . . . . . . . . . . . . . 146

A.3.2 CCE1 results . . . . . . . . . . . . . . . . . . . . . . . . . 149

B Case Study 2 Data 151

B.1 Application validation . . . . . . . . . . . . . . . . . . . . 152

B.1.1 Application validation form . . . . . . . . . . . . . . . . 153

B.1.2 Application validation results . . . . . . . . . . . . . . . . 154

B.2 Tool selection . . . . . . . . . . . . . . . . . . . . . . . . . . . . . . . . 157

B.2.1 Tool selection forms . . . . . . . . . . . . . . . . . . . 158

B.2.2 Tool selection results . . . . . . . . . . . . . . . . . . . . . . . . 159

B.3 Cross-company evaluation $2 \ldots \ldots \ldots \ldots 16 \ldots$

B.3.1 CCE2 forms . . . . . . . . . . . . . . . . . . . . . . . . . . 161

B.3.2 CCE2 results . . . . . . . . . . . . . . . . . . 165

$\begin{array}{ll}\text { C Case Study } 3 \text { Data } & 169\end{array}$

C.1 Application validation . . . . . . . . . . . . . . . . . . . . . . . . . 170

C.1.1 Application validation form . . . . . . . . . . . . . . . . . . 171

C.1.2 Application validation results . . . . . . . . . . . . . . . . . . 174

C.2 Cross-company evaluation $3 \ldots \ldots \ldots$

C.2.1 CCE3 forms . . . . . . . . . . . . . . . . . . . . . . . . . . . . . . 179

C.2.2 CCE3 results . . . . . . . . . . . . . . . . . . . . . . . . . . . . . 182 


\section{$1 \circ$ Introduction}

A challenge inherent to the nature of product development is the lack of concrete design information while making decisions. Without making decisions, designers can never transform a product idea into a (physical) product; at some point a decision has to be made regarding e.g. the dimensions, the material or the functionality of a product. While some decisions seem straightforward or trivial, consider the development of totally new, complex or interactive products; decisions become much more difficult to make as their consequences are more difficult to predict and oversee. Making a wrong decision may cause redesigns to be required (when the mistake is noticed in time), or it might cause the product to fail in the market (when the mistake is noticed too late). For example, when designing a new car, even the smallest decision might affect the 'driving experience' of the final design. A wrong design decision in any stage of its design may prevent the car from becoming a market success.

The earlier design information is available to product designers, the more effectively it can be used to adjust the course of the design process without requiring significant redesign efforts. Consequently, one of the major challenges of product designers is to gather as much reliable design information as early as possible. This thesis addresses this challenge by investigating the use of Virtual Reality (VR) to facilitate product designers in eliciting reliable design information from end-users.

\subsection{User Centred Design \& Virtual Reality}

The User Centred Design (UCD) philosophy advocates a central role for end-users within the development process. Involving end-users in the early stages of the development process allows product designers to ask what end-users think of a product concept, see how end-users would use a product concept or even ask end-users to assist in the definition of a product concept. Traditionally, designers use sketches, prototypes and mockups to facilitate communication with end-users. However, when developing new, complex or interactive products, these design artefacts fail to fully convey the product, interactions and use context. It will for example be difficult for end-users to reflect on the expected driving experience of a new car by showing them a sketch of the steering wheel.

The current research proposes to use VR technologies to enable product designers to create realistic representations of future products, user-product interactions and use contexts. VR technologies create an alternative reality in which worlds, objects and characters can be experienced that may not yet be available in reality. It allows end-users not only to see the future product (which could also be achieved with a concept sketch or mockup), but also to experience the product by interacting with it. This high-fidelity interaction with product concepts is expected to elicit more profound insights and feedback from end-users, and consequently 
helps product designers with creating products that suit end-user needs and expectations. In the example of a new car, end-users could use a driving simulator to feel what it is like to drive the new car, and consequently provide car designers with feedback on how the current design could be improved.

\subsection{Problem description}

UCD activities have the most significant impact during the early stages of the design process. The earlier design information is elicited from end-users and made available to product designers, the easier it is for product designers to use this information to make the right decisions. Consequently, the primary challenge of using VR to facilitate UCD activities is to provide endusers with a representation and experience of the future product, in a very early stage of the design process.

While numerous VR applications have already been established in the product development domain (e.g. virtual prototyping, virtual manufacturing and simulations), these applications primarily take place in more advanced stages of the design process. Deploying $V R$ in the early stages of the design process introduces several challenges. For example, due to the lack of concrete design information a detailed product model (e.g. a CAD model) is usually not yet available. Furthermore, the early stages of the design process typically involve non-expert stakeholders who may not be used to working with VR applications or design tools in general. Lastly, the lack of structure in the early stages of the product development process (PDP) creates a need for flexible (in terms of functionality) and easily deployable (in terms of usability) tools to conduct design activities. The tools currently available to facilitate design tasks through $\mathrm{VR}$, however, expect designers to have programming experience, to invest in training, or to use the tools on a regular basis.

In other words, neither the VR applications nor the tools currently offered to the product development domain are considered appropriate for facilitating UCD activities.

The research presented in this thesis addresses this issue.

- Firstly, it identifies and develops advantageous VR applications that specifically facilitate UCD activities.

- Secondly, it will determine the boundary conditions for realising these applications within UCD practice.

Both aspects are first investigated in a specific design context by conducting several industrial case studies. Then, by evaluating the specific insights across various design contexts, generic insights regarding the facilitation of UCD through VR are obtained.

\subsection{Context}

With its roots in computer science and computer graphics, VR has primarily been described from a technological point of view. The resulting technological developments led to the adoption of VR in various domains, including product development. Application oriented surveys, such as the study of Jimeno and Puerta (2007) and the work of Ye et al. (2006), illustrate examples of VR being used in this domain to facilitate 3D modelling, virtual prototyping, virtual assembly and manufacturing or virtual training. For the product development domain, one of the primary benefits of $V R$ is that it allows non-existing products and environments to be experienced in a natural and realistic way. This is beneficial when the real world situation is too dangerous 
(e.g. a drive simulator, as described by Tideman et al. (2008)), when an environment needs to be controlled (e.g. in simulation and evaluation as described by Kuutti et al. (2001) or when physical prototyping is too expensive or simply not yes possible (e.g. virtual prototyping, as described in Balcisoy et al. (2000)).

However, as explained in the introductory section, in spite of their contributions to the product development domain in general, these established applications are not expected to be appropriate for facilitating UCD activities in the early stages because they support a different type of design task (the application) and do not provide appropriate means (tools) for designers to realise the application themselves. One of the first aims of the current research, therefore, is to determine an approach for identifying advantageous applications of VR in the context of UCD, and for identifying requirements with respect to the tools needed to realise these applications.

In literature two types of approaches for the identification of advantageous VR applications can be found. The 'technology driven' approach originates from research in the field of VR technologies and aims to identify useful applications of a given VR technology, such as augmented reality or immersive VR. While this work leads to concrete applications, the results tend to be limited to case specific implementations of a particular technology, rather than providing general guidelines for applying VR in design practice. The second approach is referred to as 'application driven', and identifies advantageous applications of VR by looking for VR technologies that facilitate a given design task or objective. Miedema (2010) for instance developed a generic framework to not only identify the added value of VR in a PDP, but also discuss how the technology should be integrated in the existing development process. A consultancy solution is proposed in which product designers are given advice on what type of synthetic environment (an application of VR) would be beneficial for a specific product development activity.

The application driven approach is considered appropriate for the identification of useful and feasible applications of VR in the PDP. However, even though the work of Miedema (2010) explicitly addresses the early stages of the PDP there are some limitations that justify further research. For instance, the selection framework depends on the availability of existing VR solutions for facilitating UCD, while current solutions primarily target more advanced stages of the PDP. Furthermore, it is questionable whether a consultancy approach is also appropriate for UCD activities. A closer look at the characteristics of UCD activities (especially in relation to other conceptual design activities) is therefore required to refine this approach for the current research.

Compared to the identification of advantageous applications of VR, the identification of tool requirements (or the boundary conditions within which a VR application is to be realised) is less extensively covered by literature. While the development process of VR applications has been described technically, for instance by Eastgate (2001) and Fencott (2004), these structures focus on the outcome of the development process but do not take the development context (e.g. who is realising the VR application) into account. Even user centred development proposals such as those described by Bowman et al. (2002) or Kaur (1998) only involve endusers to evaluate the usability and user experience of the application, but not the usability and experience of creating the application. A more appropriate solution is to actively include the end-users of the resulting VR application in the selection and realisation phase of the application, as presented in Crosier et al. (2002). In the work, teachers are involved in the development of an educational VR application, in the end making sure that the resulting application not only serves a useful purpose, but is also usable by the teachers and students. A similar approach is desired for the current work; product designers are to be involved in the selection and evaluation of the tools that eventually enable them to realise the anticipated VR application themselves. 


\begin{tabular}{lll}
\hline & Products & Participating department \\
\hline Company A & Professional office equipment & Design department \\
Company B & Heavy-duty commercial vehicles & Vehicle definition department \\
Company C & Food processing solutions & R\&D department \\
\hline
\end{tabular}

Table 1.1 Overview of the three industrial partners and the departments directly involved in the research.

\subsubsection{Practical context}

The practical context consists of the research project of which the current work is part. The REPAR project (Resolving the Paradox in User centred Design ${ }^{1}$ ) investigates various means to support flexible prototyping in the early stages of a UCD process. The project aims to provide tools and methods that (together) support the creation of prototypes ranging from low-fidelity sketchy prototypes to high-fidelity virtual prototypes.

The research project involves three industrial partners, as listed in table 1.1. All three partners are medium sized to large multinational companies. While the companies operate in different industries, their focus is on the development of complex (i.e. involving a high number of interdependent and multi-domain components) and interactive (i.e. the products directly or indirectly respond to end-user input) products. Throughout this research the researcher will collaborate with members of the design (or R\&D) departments of these companies and carry out a case study within the context of each company.

\subsubsection{Focus}

Within the broad theoretical context, an approach is defined for identifying advantageous applications of VR that facilitate UCD activities, and the identification of tools that enable the realisation of these applications. As UCD activities primarily affect the early stages of the PDP, the focus of the research is limited to these stages, involving all successive design activities and iterations from ideation up to the point of a concept freeze. As further discussed in section 2.2.3, the search for advantageous applications (and supporting tools) will focus on low-end and off the shelf VR techniques, improving the availability to design practice and reducing the complexity of the tools needed to realise the applications.

During the industrial case studies, the research scope is temporarily limited to the respective companies; the researcher acts as a 'VR consultant' and focuses on the specific requirements and constraints imposed by the company involved in the case study. In the final stages of the research the focus is on providing design practice with generic insights regarding the application and realisation of VR applications in various design domains. Here the role of the researcher is no longer that of a VR consultant but that of the design researcher investigating whether different design domains can benefit from the same applications, and how different design domains affect the boundary conditions for the realisation of the applications.

\subsection{Objective}

The objective of the research is to provide insights in the feasibility of VR as a means to facilitate UCD tasks in the early stages of a product design process. Here feasibility depends on

\footnotetext{
${ }^{1}$ See http://www.repar-project.com
} 
the identification of advantageous applications of VR as well as the identification of appropriate tools for design practitioners to realise these applications.

Both aspects are first investigated in a specific design context by conducting industrial case studies, involving the identification, development and evaluation of UCD oriented VR solutions. Then, by evaluating the specific insights across various design contexts generic insights regarding the facilitation of UCD through VR are obtained. The resulting insights help design practitioners with assessing the contribution of virtual reality to a particular design task, and with determining boundary conditions for realising the desired application.

\subsubsection{Contributions}

The main contribution of the research consists of generic insights in advantageous applications of VR in a UCD process and the realisation of these applications through appropriate tools. This result is new in the sense that 1 ) current work primarily describes applications of VR in the PDP in general, rather than specifically addressing UCD tasks, and 2) because existing work provides either case specific insights or generic insights in advantageous applications without providing insights into how the applications are to be realised within the boundaries of a particular part of the PDP.

The insights can be used 1 ) by design practitioners to select tools that help realise a desired application, 2) by design researchers or consultants aiming to facilitate this selection process and 3 ) by software developers aiming to provide VR design tools that specifically target UCD tasks.

Furthermore, the research contributes to design practice by providing three concrete case studies that illustrate how VR can be used to facilitate UCD tasks in the early stages of the PDP. Practitioners can use the case studies for inspiration on how to deploy VR effectively, or use the application prototypes and accompanying tools to directly deploy the application in their own practice.

The developed methods used for the definition, development and deployment of VR applications and tools can be used by design researchers to further extend the body of knowledge regarding the use of $V R$ in $U C D$, but also by design practitioners to apply it to their own specific setting.

\subsection{Thesis outline}

This thesis comprises three parts, describing the research background and approach, the proceedings of the company specific case studies and the generalisation of insights and reflection on the research approach and methods.

The first part contains chapters 2 and 3 . Chapter 2 elaborates on the theoretical context in order to explain why VR is believed to be beneficial to UCD practice, how existing work does not provide the right type of applications and tools for this specific type of design activities, and to provide a first outline of the research approach. Chapter 3 elaborates on the research approach by describing the structure of the research approach and the design of methods that respectively support the specification and the generalisation phases of the research.

The second part of the thesis, containing chapters 4,5 and 6 , presents the proceedings of the three industrial case studies carried out within the scope of this research. For each industrial partner, the chapters describe the identification, development and evaluation of a specific VR application and the subsequent selection and evaluation of supporting tools. 
In the third part of the thesis, chapters 7 and 8 respectively describe how the results of the case studies were translated into generic insights regarding the application of VR in UCD practice, and reflect on the methods used to achieve this. The generic insights presented in chapter 7 support design practitioners in determining how VR can facilitate UCD activities in their design context. Chapter 8 offers design researchers insights into the strengths and weaknesses of the research approach, and provides recommendations regarding the implementation of the methods presented in this work. Chapter 9 concludes the research by summarising the key findings, discussing the validity of the work and by outlining future work. 


\section{$2 \circ$ Background}

This chapter elaborates on the research context that was outlined in chapter 1 . Section 2.1 describes UCD and its position and role in the design process and highlights characteristics and challenges relevant for the present research. Section 2.2 covers VR, focusing on the use of $\mathrm{VR}$ in the product development domain by discussing several existing applications. Section 2.3 presents the results of a field study that investigated the current state of UCD and VR in the design practice of the industrial partners involved in the research project. Section 2.4 discusses the findings of the literature research and the field study and concludes with directions towards a research approach for the current work.

\subsection{User Centred Design}

The UCD philosophy originates from the 1980s, when the increasing use of machines and computer systems triggered system designers to start thinking about usability aspects, such as the ease of use and the ease of learning. Until then, systems had been designed for optimal performance, rather than usability. The work of Norman (1986) and Norman and Draper (1986) is generally referred to as the origin of 'user centred system design'. As Norman (1986) states:

"[...] user-centred design emphasises that the purpose of the system is to serve the user, not to use a specific technology, not to be an elegant piece of programming."

In The Psychology of Everyday Things (Norman (1988)) Norman further elaborated on this philosophy, providing guidelines on how systems could be designed in a user centred fashion. In the same year Gould (1988) presented a more process oriented set of principles, which not only gives the user a central role in the design process but also stresses the importance of doing user testing, iterative design, and integrated design. The purpose of giving end-users a central role in the design process is for designers to gain insights in relevant end-user needs and consequently design products (machines, computers, etc.) that meet these needs. Especially when designing products that are used in various use contexts (or dynamic use contexts, as discussed by van der Bijl-Brouwer (2012)) (e.g. a mobile phone, which can be used for personal as well as professional purposes) or that face multiple types of users (e.g. an ATM machine which should be accessible to a wide range of end-users), it is important for product designers to take the user's objectives and needs into account.

The basis of UCD has been formalised in the 'ISO 9241-210 standard on human-centred design processes for interactive systems' (DIS (2010)) $)^{1}$. The standard describes six principles

\footnotetext{
${ }^{1}$ This standard replaced ISO 13407 in 2010, see Travis (2011). The four principles of UCD originally presented in ISO 13407 (as discussed by Maguire (2001)) have been extended to the current set of six.
} 
of UCD as follows;

1. The design is based upon an explicit understanding of users, tasks and environments.

2. Users are involved throughout design and development.

3. The design is driven and refined by user-centred evaluation.

4. The process is iterative.

5. The design addresses the whole user experience.

6. The design team includes multidisciplinary skills and perspectives.

In spite of the availability of the ISO standard, the UCD philosophy evolved into a term that describes any design process related to involving end-users (see Gulliksen et al. (2003), and livari and livari (2011)). Although Karat (1996) questions whether or not this lack of a shared understanding and meaning of UCD is actually a problem, it is considered useful to further define the interpretation of UCD in the current research.

The UCD principles defined by the ISO 9241-210 standard form the basis of what is considered UCD in this research. Originating from the field of human-computer interactions, UCD is traditionally associated with the design of interactive systems, services and graphical user interfaces. Surveys such as those reported by Maguire (2001) or Vredenburg et al. (2002) illustrate the established and growing adoption of UCD principles in the field of human-computer interactions. The current research however focusses on the user centred development of physical products, in which similar principles can be used (as also described in The Design of Everyday Things by Norman (2002)). While the principles are the same, the terminology is often different from terminology used in the field of human-computer interactions. As Kaulio (1998) describes;

"User-oriented product development [...] is characterized by a problem analysis of user/use requirements with a starting point in the use situation, leading to the formulation of 'user requirements', a transformation of these user requirements into measurable engineering requirements, and an iterative design where prototypes are tested by users and modified by designers".

The use of different terminology (e.g. user-oriented vs. user centred) is not always a trivial matter. Veryzer and Borja de Mozota (2005) explicitly call it 'user oriented' instead of 'user centred' to emphasise that user centred design may sound too restrictive for design processes that inherently have some degree of 'technology push'. While not neglecting the importance of apt terminology, for the sake of clarity and consistency the current work uses the term User Centred Design to refer to the application of UCD principles to the PDP of physical products. To further outline UCD and its role in the PDP, the following two subsections elaborate on the PDP and on the involvement of end-users in it respectively.

\subsubsection{The product development process}

The PDP (product development process or design process) consists of a sequence of stages in which a product evolves from an idea or product concept into a physical product. Ulrich and Eppinger (1995) provide a generalised description of design stages, consisting of

1. a conceptual design stage in which an initial product concept is defined, 
2. a detailed design stage in which the product concept is further developed,

3. an engineering stage in which the product is made ready for manufacturing,

4. a manufacturing stage in which the product is actually created, and

5. a market release stage in which the product is 'released'.

Within and between these stages numerous iterations of concept generation, concept testing and concept re-design take place. An extensive discussion of design activities taking place in various stages of the PDP is presented in the work of Krishnan and Ulrich (2001).

The exact number, duration and nature of PDP stages depends on the product being developed, the type of development process and which design methods and tools are used;

- Complex products, such as products with a high number of parts, or products that integrate various technical domains (e.g. mechatronic products) generally take more time to develop than simple products (e.g. single component products).

- The development of totally new products (e.g. radical or break-through innovation) generally takes more time than incremental or platform-based development because the development process is more difficult to predict and manage.

- Design methods and tools aim to support design activities by directing the course of a design stage (design methods) and by supporting the designers in carrying out these directions (design tools).

While proceeding through the various stages of the PDP, product designers gather design information about the product being developed, such as its main functions, the exact dimensions or the materials used. In return for design information, it becomes more and more difficult (i.e. time taking and expensive) to make changes to the design. Consequently, one of the major challenges in the PDP is to gather as much reliable design information as early as possible. End-users provide a valuable source of design information. According to the UCD philosophy, they can be considered as 'experts in use', and should therefore be involved in the development process. The next subsection elaborates on methods for involving end-users in the PDP.

\subsubsection{User involvement in the product development process}

To successfully involve end-users, the PDP needs to have appropriate facilitating characteristics. Tideman et al. (2008) propose the following set of conditions for effective and efficient participation of end-users.

1. Direct and explicit communication between designer and end-user needs to be established. The means of communication should minimise the chance of misinterpretation on either side.

2. End-users should be enabled to have a realistic interaction with the design information. They should be able to reliably assess the exact functioning and experience of the design under a wide range of circumstances.

3. End-users should be enabled to reliably become conscious of and assess the consequences of design decisions. Consequences of design decisions should be made explicit and presented in a manner that is comprehensible regardless of the participant's training or discipline. 
There is a wide range of tools and methods that provides concrete implementations of the principles by facilitating communication between product designers and end-users. Specific implementations differ in when and how end-users are involved in the PDP. In a survey of user ${ }^{2}$ involvement in the PDP, Kaulio (1998) describes these dimensions as points of interaction and depth of end-user involvement respectively. In principle, end-user involvement can take place throughout the PDP, but as also concluded in this work, the activities primarily take place in the early stages. The depth of end-user involvement refers to how end-users are involved in design activities. Kaulio (1998) defines three levels of involvement (quoted):

1. Design for: denotes a product development approach where products are designed on behalf of the customers. Data on users, general theories and models of customer behaviour are used as a knowledge base for design. This approach often also includes specific studies of customers, such as interviews or focus groups.

2. Design with: denotes a product development approach, focusing on the customer, utilising data on customer preferences, needs and requirements as in a 'design for' approach, but, in addition, includes display of different solutions/concepts to the customers, so the customers can react to different proposed design solutions.

3. Design by: denotes a product development approach where customers are actively involved in and partake in the design of their own product.

In practice, UCD activities can be very specific (i.e. target one specific level of user involvement, and one point of interaction), or more flexible (i.e. cover multiple levels of involvement or support more than one point of interaction). The following paragraphs list concrete examples that have been selected to illustrate the various levels of user involvement and the techniques available to achieve this.

\section{User analysis}

Techniques such as focus groups (see Kitzinger (1995)) and context mapping (see Visser et al. (2005)) provide insights into current product use and use context. These methods have a relatively low level of end-user involvement, as end-users only perform the role of information source for product designers. Results of these activities are typically shared through reports, presentations or user profiles.

\section{Usability evaluations}

Traditionally usability evaluations have been a popular technique for reviewing the usability of new interactive systems (in particular user interfaces) with end-users in various stages of the PDP (see Nielsen (1994)). Depending on the design stage in which the evaluation takes place, functional prototypes (see Ballagas et al. (2003), Lee et al. (2004)), paper mockups or simulated ('wizard of oz') prototypes (see Bernsen et al. (1994)) can be used. The level of user involvement is usually quite high, as end-users are asked to test the new products, as well as to provide feedback on them.

\footnotetext{
${ }^{2}$ Although Kaulio (1998) uses 'customer' as a synonym for 'user' ("In this paper, the word 'customer' is employed as a synonym for consumer or user."), it should be noted that in practice, there can be an important difference between customers and end-users. In the consumer market, the customer is usually the end-user. In business to business markets, the customer is usually not the end-user.
} 


\section{Scenario based product design}

Originating from the work of Carroll (1995), scenario based product design methods use scenarios to describe current or future use situations and provide end-users with a reference point through which they evaluate a new situation (e.g. a product or use situation). This can be further supported by tangible means, such as a miniature version of the future product, a miniature context of use, or by telling (see Buskermolen and Terken (2012)) or acting out future use situations (see Bødker (2000), and Brandt and Grunnet (2000)). Scenarios can potentially facilitate various levels of user involvement, ranging from relatively low (when scenarios are presented to end-users as a means of concept presentation) to high (when created by end-users themselves).

\section{Participatory design}

Participatory design, as described by Greenbaum and Kyng (1991), is a form of UCD in which relevant stakeholders (such as end-users) actively participate in and contribute to the design process. Active participation of end-users was also advocated by Bannon (1991) in order to discover real user requirements. In participatory design, end-users work in multi-disciplinary groups to collaboratively discuss and evaluate design proposals. These sessions facilitate the gathering of design information by reviewing the design problem from various (equally relevant) perspectives. To facilitate communication and collaboration between these stakeholders, participatory design often involves low-fidelity artefacts such as cards or games (see Brandt (2006), and Garde and van der Voort (2012)). As end-users (and other stakeholders) are directly involved in the design activities, the level of end-user involvement is considered to be quite high.

\subsubsection{Characteristics of UCD activities}

The common denominator within the variety of UCD tools and methods is that a communication channel between the product designer and end-user is established. Unlike product designers, endusers are not trained to work in multi-disciplinary teams and interpret (incomplete) information from various sources. As described by Dix and Gongora (2011), designers need to provide endusers with information through appropriate means, and end-users need appropriate means to externalise their ideas and opinions. The manifestation of this representation varies from visual or written scenarios to interactive prototypes or participatory design games.

The type and fidelity of the manifestations should be a balance between minimising the time required by product designers to prepare or create the artefacts (i.e. mockups, functional prototypes or sketches) and making sure that an end-user is provided with a sufficiently concrete representation of a future product in order to give relevant feedback. If the representation is too detailed, the end-user may assume that it is no longer possible to make significant changes to the design and consequently hold back information. This could for instance happen when showing an end-user a photo-realistic render of a CAD model. Sketchy or coarse product representations make clear to the end-user that the design process is still in progress, allowing for feedback and comments from the end-user. On the other hand, a sketchy representation of a product the end-user is not familiar with is unlikely to elicit detailed feedback.

The balance between artefact fidelity and time investment can be improved by iteratively applying a technique, while constantly 'upgrading' the artefact. For example, usability evaluations typically use sketches and drawings in the very early stages of design and proceed to digital mockups and functional prototypes in advanced stages. Likewise, scenario based methods can proceed from coarse scenarios (written or storyboard) to fully animated or recorded versions 
of final scenarios. The flexibility in the application of techniques allows for iteration, but also makes UCD activities sufficiently agile for the unstructured early stages of the PDP.

The following list summarises key characteristics of UCD techniques.

1. UCD techniques establish a communication channel between product designers and endusers,

2. they are flexible, allowing designers to fine-tune the manifestation of a technique to a specific purpose, and

3. they can be applied iteratively, allowing for artefacts to be improved in each iteration.

This review of UCD in the domain of physical product development illustrated the particular challenges of the early stages of the PDP, and identified characteristics of tools and methods that contribute to a successful implementation of UCD principles.

\subsection{Virtual Reality}

The publication of Sketchpad by Sutherland (1968) is often cited as the start of virtual reality. The work describes the projection of a 3D model on a head mounted display that changes perspective based on the movement of the user. As a result, the illusion of 'presence' within that projection is created. Since its initiation, VR evolved in two directions. Firstly, technological developments in the field of virtual reality have improved and extended the virtual world in which users are immersed, for instance by creating higher resolution head mounted displays, 3D audio solutions or improved haptic (touch) devices. Technological developments in turn expanded the application domain of VR to design and engineering, healthcare, military, entertainment and education.

The following sections describe the adoption of VR in the product development domain, particularly focusing on the conceptual or early stages of the PDP. Section 2.2.1 first provides a definition of VR and an overview of underlying technologies. Section 2.2.2 discusses various existing applications of VR in the PDP. Section 2.2.3 presents trends and opportunities that are relevant in the context of this research.

\subsubsection{Definition \& overview}

VR is multifaceted in terms of application domains as well as underlying technologies. As a result, there are many different definitions that describe VR from specific perspectives. Miedema (2010) (pp. 9-11) for example defines generation, control, perception and value as perspectives from which VR can be interpreted.

Cobb et al. (1995) make a distinction between VR and Virtual Environment (VE), and refer to VR as the technology used to create VE's. An important aspect of this definition is that there is no restriction as to what is (or is not) considered a VR technology, but only as to what the resulting VE should achieve;

"What is important is to create experiences that appear and behave credibly, consistently and coherently, and that allow participants to relate the experience to the real world. The essence of the VE then is that it should enable participants to feel displaced to a new location and interact with that environment and the objects within it, and they should feel that the objects they are manipulating or observing are behaving appropriately." 


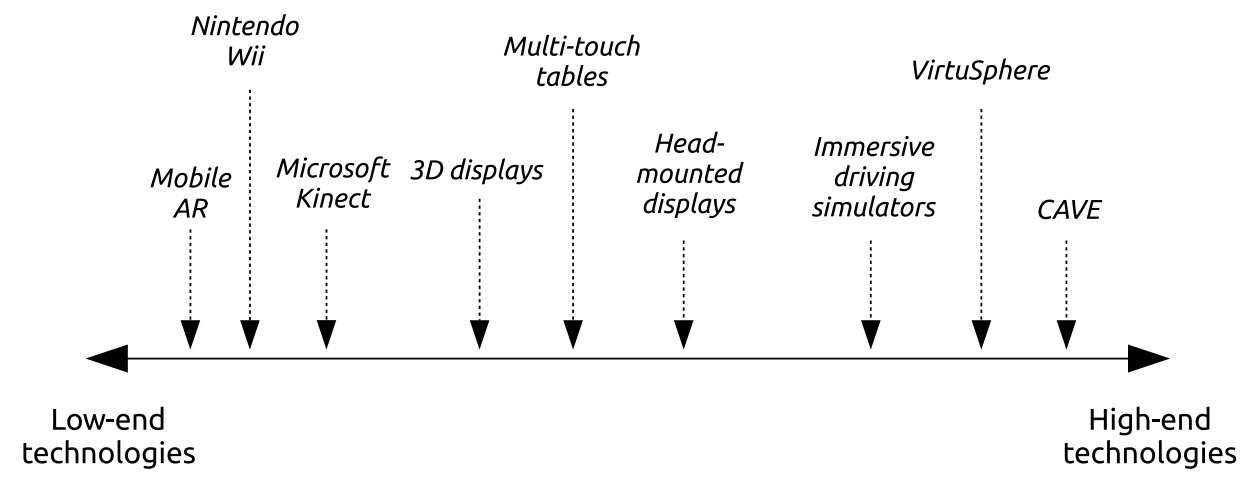

Figure 2.1 Examples of low-end and high-end VR technologies.

A more compact definition of this interpretation is given by Cobb and Sharkey (2007), who defines the VE as "computer generated three-dimensional environments that can be explored and interact with in real-time". This definition suits the current research because it considers the purpose of the VE to be more important than its technical implementation, and because it makes a clear distinction between what the VE is used for (e.g. the application aspect), and how the VE is created (i.e. the tool aspect).

\section{Underlying technologies}

There are many ways to characterise or structure the underlying technologies of VR. Multiaspect structures such as the one described by Blach (2008) provide a solid basis for the classification and comparison of virtual environments and related technologies, based on (among others) the degree of embedding physical reality, the level of collaboration and the fidelity of content. Another structure is described by Milgram and Kishino (1994), who define the RealityVirtuality continuum by stating that "[...] real environments and virtual environments are not to be considered simply as alternatives to each other, but rather as poles lying at opposite ends of a Reality-Virtuality continuum, [...]". Placing existing types of virtual environments representing various degrees of embedding the physical reality in this continuum provides a good overview of technologies involved in VR.

While these structures provide an appropriate basis for the characterisation and comparison of VR technologies, the current work uses a more pragmatic approach in describing technologies that reasons from the perspective of product designers who eventually have to acquire and use the technologies. The characterisation used in the current work is therefore primarily based on the availability of the technologies on the market, represented by low-end and high-end technologies, as illustrated in figure 2.1.

- Low-end - Low-end technologies are available as commodity hardware and software, and can often be deployed off the shelf. The technologies generally support a broad range of applications, and provide room for customisation through end-user configuration software or API's for developers. Examples of low-end technologies include modern game plat- 
forms such as the Nintendo Wii ${ }^{3}$ and Microsoft Kinect ${ }^{4}$, augmented reality hardware and software and 3D displays.

- High-end - High-end VR technologies are not available on consumer markets but are specifically developed for professional applications. They typically have a more significant initial investment, and require professional support for the installation, use and maintenance of the manifestation. Furthermore, they either serve a very specific application or require additional development to support custom applications. Examples of such technologies include advanced driving simulators, CAVE environments ${ }^{5}$ and the VirtuSphere ${ }^{6}$.

Compared to the other characterisation structures, an important difference is that the structure used in the current research is based on the properties of the software and hardware required to realise and operate the VR manifestation (e.g. price or required support), rather than the manifestation itself (e.g. fidelity or the level of immersion). There is no fixed relation between the availability of a technology and the properties of a manifestation. For example, high-end technologies do not automatically provide a high-fidelity virtual environment or a high level of immersion.

\subsubsection{Applications in product development}

Over the years, VR technologies have found their way into the realms of the PDP. Initially VR was primarily used by industries involved in the manufacturing of large complex machines, such as automotive, aerospace, healthcare and the military. By combining VR technologies with established $C A x^{7}$ systems, various applications emerged, such as virtual prototyping, virtual engineering, virtual manufacturing and virtual training. Weidlich et al. (2009) provide an extensive overview of VR applications in design and engineering.

In the current research, the focus is on the early stages of the PDP. The following subsections present several example applications of VR in the early stages of the PDP. The examples have been structured according to their role in the PDP, namely concept generation, concept presentation and concept evaluation.

\section{Concept generation}

Concept generation involves the externalisation of a product idea into a product concept that can then be presented or evaluated by a design team. VR primarily supports concept generation tasks by facilitating the creation of virtual prototypes. Here the role of VR is to provide easy to use model manipulation interfaces (e.g. haptic input devices). Making the manipulation easier not only helps designers in quickly creating virtual prototypes, but also potentially allows untrained stakeholders such as end-users to participate in the generation of concepts.

Ye et al. (2006) describe a virtual prototyping tool called LUCID (Loughborough University Conceptual Interactive Design). The tool consists of a VR CAD application that allows designers to "(...) take full advantage of their visual, auditory and tactile channels in order to create, view, touch, manipulate and listen to CAD models (...)". The software was tested by product designers who indeed preferred the innovative $C A D$ interfaces over the traditional mouse and

\footnotetext{
${ }^{3}$ Nintendo's game console that supports 3D input devices, see http://www.nintendo.com/wii

${ }^{4}$ Microsoft's imaging based motion sensing device, see http://www.xbox.com/kinect

${ }^{5}$ Cave Automatic Virtual Environment, see http://en.wikipedia.org/wiki/Cave_automatic_virtual_environment

${ }^{6} \mathrm{~A}$ locomotion platform that allows users to be completely immersed, see http://www.virtusphere.com

${ }^{7}$ Computer Aided Engineering, Manufacturing, Design, etc.
} 
keyboard. The work in Bordegoni and Cugini (2006) describes the development of a haptic tool that supports designers and modellers during early stages of design. The tool allows modellers to sculpt, inspect and refine a virtual model by using a haptic tool that resembles traditional tools, like cutters, sand paper, templates, etcetera. Bruno and Muzzupappa (2010) present a virtual prototyping tool for product user interfaces, meant to be used in participatory design tasks.

\section{Concept presentation}

Concept presentation involves the presentation of new product concepts to relevant stakeholders, typically with the aim to elicit discussions and feedback from the stakeholders. Various VR applications have been developed to facilitate concept presentation activities, primarily because VR allows designers to present their concepts in a way that is closer to reality than for instance a sketch or a static digital visualisation, and because it allows a product to be presented in a realistic use context. This makes it easier for external stakeholders to interpret the concept, and to provide relevant and rich feedback. Furthermore, VR also addresses issues related to geographical distances, as it allows stakeholders to collaborate remotely over the Internet.

Over the last decade various proposals of VR collaboration frameworks and tools have been presented. In Kan et al. (2001) a collaborative discussion and evaluation system (VRCE) is presented. This system is based on standards such as VRML and Java, allowing regular web browsers to be used for virtual collaborative design sessions in which concepts are discussed. As with other work in this field, the focus is on exchanging rich data over large networks such as the Internet. Other examples presenting similar solutions include CyberReview by Huang (2002) and TEAM by May and Carter (2001). Apart from enabling remote collaboration, these VR applications also facilitate interaction with the product concept (and sometimes its context) and prevent misinterpretations by providing a more explicit representation of the product concept. A survey of other work in this field is provided by Wang et al. (2002).

\section{Concept evaluation}

During concept evaluations, external stakeholders, such as end-users, are involved to try out new product concepts and to provide feedback to help product designers improve the product. VR can support these activities in various ways. For example, the realistic presentation of a concept (as seen in the previous paragraph) can help stakeholders with interpreting new product concepts. Furthermore, when interactive virtual prototypes are available, stakeholders can actually experience the new product concept rather than just see it.

This is particularly useful when the use situation that is evaluated does not exist yet, or is a potentially hazardous one. For example, it would not be safe to evaluate the first prototype of a new driver-assistance system in a real-life situation. A realistic driving simulator on the other hand provides a safe and controllable testing environment. Another benefit of using VR for concept evaluations is that it allows for designers to efficiently test various alternatives, and ask test participants to change or optimise the design while they are evaluating it. For example, Bruno and Muzzupappa (2010) present a virtual prototyping tool for product user interfaces that allows participants to modify the user interface during the tests. Other potential advantages of using VR to facilitate concept evaluations, as described by Kuutti et al. (2001), include the ability to present an integrated product (i.e. the test participant is shown a realistic and integrated version of a product, rather than a sketch of the physical product and a digital prototype of its user interface), and the ability to conduct remote concept evaluations, where the 
participant is geographically separated from the designer. This could for instance be beneficial when product designers want to cover an international representation of product users.

\subsubsection{Trends \& opportunities}

Especially over the last decade, both the development and the application of VR technologies dissipated across multiple domains and industries and are no longer confined to research or large industries. The gaming and entertainment industry for instance successfully adopted several VR technologies and implemented them in consumer products, such as the Nintendo $\mathrm{Wii}^{8}$, the Microsoft Kinect ${ }^{9}$ and Sony's Playstation Move ${ }^{10}$. The use of these technologies in consumer products stimulates technological refinements, and familiarises consumers with new technologies. Other relatively new technologies, such as multi-touch displays are now a commodity because of their application on smartphones and tablet computers.

Improvements of supporting infrastructures (e.g. graphics hardware, software platforms, data communication) have further facilitated the maturing of traditional VR technologies, such as high-end visualisations that no longer require special hardware, and augmented reality which now runs on mobile platforms and uses off the shelf authoring software. The availability of fast and reliable data communication (e.g. over the Internet) has facilitated the generation and exchange of virtual assets, such as 3D models, textures or entire virtual worlds. Virtual assets no longer need to be created from scratch, but can be retrieved from (online) databases.

Another interesting trend related to the supporting infrastructure is that hardware and software manufacturers can ask consumers to explore and possibly improve their products. For example, numerous improved or modified versions of the Kinect and the Wii software have been published online, leading to several useful prototypes and applications. These consumer market game platforms have even served as prototype hardware in various research projects regarding e.g. interaction design, as described by Schreiber et al. (2009).

\subsubsection{Discussion}

In spite of targeting the early stages of the PDP, most of the VR applications presented in this section more or less rely on data provided by CAD systems. While it is not surprising to see a strong relation with this established design tool, it can be argued that simply extending CAD functions to the early design stages of the PDP is not recommended. Ottosson (2002) mentions two reasons why CAD tools are not suitable for early stage design. Firstly, CAD tools force engineers or designers to work on a very detailed level from the start; in the early stages of development. As Ottosson (1998) indicates;

"CAD programs force engineers to build products up from exactly defined details and not from the totality of the concept down to the details of the product. [...] functional thinking on an abstract level without taking into account dimensions is initially very important"

Secondly, CAD tools primarily help with dimensions, but lack support for other aspects such as interactions with users, visual realism or use context. Furthermore, in the context of the current research it is argued that using CAD as a basis (or even starting point) of the application causes the application to focus on mediation between the operator (the designer or end-user)

\footnotetext{
${ }^{8}$ Nintendo's game console that supports 3D input devices, see http://www.nintendo.com/wii

${ }^{9}$ Microsoft's imaging based motion sensing device, see http://www.xbox.com/kinect

${ }^{10}$ Sony's motion sensing game controller, see http://www.playstationmove.com
} 
and the CAD model instead of facilitating the communication between end-users and designers. The high level of fidelity and detail in CAD models is more likely to make end-users think the design has already been 'frozen', which may prevent them from questioning the principles of the concept, but rather focus on providing detailed feedback on the model.

Applications that let go of the CAD basis, such as the use of VR to facilitate group collaborations, or the use of VR to create early stage GUI prototypes, are less constrained by limitations such as the rendering or modification of detailed geometry, or the need for exact definitions of dimensions and relations. While the artefacts used in these applications are typically less detailed, they provide sufficient means to facilitate the anticipated design tasks (i.e. concept generation, presentation or evaluation). However, the lack of an established starting point (such as CAD basis) does result in a myriad of individual VR support platforms and tools, making it more difficult for the application to be realised without specific expertise or resources. The survey of Wright and Madey (2009) illustrates this with a structured overview of past and current development support frameworks, platforms and API's. Apart from the fact that the majority of these support tools do not outlive the application or research context for which they were created, the current research also argues that none of these tools can really be used without experience in software development, which makes them unusable for (most) designers.

\section{Conclusion}

While most of the current applications of VR in the PDP take place in the advanced stages, such as the engineering and manufacturing stages, it was shown in section 2.2.2 that various useful applications in the early stages of the PDP can be found as well. Most of these applications however rely on a CAD basis to provide relatively detailed models, which are not considered very appropriate for the early stages of design. However, it was also shown that without a solid basis such as CAD, the myriad of frameworks and platforms alternatives make VR applications difficult to integrate in an existing PDP. The field study presented in the next section investigates if and how the challenge of realising VR applications is addressed in current design practice.

\subsection{Field study}

A field study was conducted with the aim to investigate the current state of UCD and the current adoption of VR (and related) technologies within the design departments of the industrial partners involved in the REPAR research project. The study involved a series of interviews, carried out during internships between November 2009 and February 2010, and a VR demonstration session. The VR demonstration session aimed to create a common understanding of $V R$ among the participating industrial partners.

\subsubsection{Interviews \& site visits}

A series of interviews (a total of 49 one hour interviews) and site visits was conducted among the industrial partners involved in the research project. During the interviews, members of the design departments and other relevant departments (e.g. marketing, sales or engineering) were questioned about their use of prototyping tools in general ${ }^{11}$, the adoption of UCD techniques, and the use of VR or related technologies during any stage of their PDP. The following statements summarise the findings relevant for the current research.

\footnotetext{
${ }^{11}$ Conform the topic of the REPAR project, see chapter 1
} 
1. While the initial visits aimed at investigating user/designer communication, it was found that a lot of issues are actually related to internal communication as well. Different (professional) backgrounds often lead to misunderstandings and design errors.

2. When investigating the current state and future need for UCD, companies in general indicated no need for 'co-design', but agree that their development process could be improved by involving the user more actively.

3. In general the first stages of development are not as well defined as the engineering and manufacturing stages, in terms of tools as well as methods. Product designers individually choose tools and methods, lengthening the process and making communication difficult.

4. Most of the interviewees found it difficult to discuss the topic of VR; there was a lack of example material, and most of the interviewees had no past experience with the technology, or a limited reference based on movies or entertainment.

As indicated by finding 4, the use of VR was found to be very limited. While the added value of VR was generally acknowledged, designers indicated that the means to create those applications should be easy to use (because designers are not computer scientists), flexible (because designers should be able to deploy VR applications in various design cases) and compatible with existing tool chains. Based on these findings, it was decided to first establish a common understanding of virtual reality and its potential application in product development.

\subsubsection{VR demonstration session}

The VR demonstration session aimed to create a shared understanding of VR by demonstrating various design related VR applications. The applications should demonstrate as much of the VR spectrum as possible (e.g. immersive VR, mixed reality and desktop VR), and they should be relevant to the participating industrial partners in the sense that the participants recognise the added value for their PDP. To achieve this, the researcher developed four VR demonstrations illustrating the application of a specific VR technology to a specific design task. Results of the interviews and site visits were used as 'use cases' for the demonstrations.

1. AR Factory Layout - This demonstrator uses Augmented Reality (AR) to configure and review factory layouts (see figure 2.2a). The application was designed to help machine designers with communicating their designs to customers. The demonstrator consists of simple 3D models of the company's products rendered and controlled in Blender ${ }^{12}$. The scene objects are connected to AR markers through ARToolkit ${ }^{13}$. Each marker contains a virtual model of a machine and also provides layered information, such as machine dimensions, machine input and output channels and hazardous areas.

2. Virtual Usability Lab - This demonstration consists of a 3D virtual office environment in which designers can walk around and operate virtual office machinery (e.g. printers, computers, etc.) (see figure 2.2b). The environment was created using Blender's game engine and projected on a large screen to create a semi-immersive experience. Designers control a first person perspective using a keyboard and mouse, and are able to carry out simple interactions with machines.

\footnotetext{
${ }^{12} \mathrm{~A} 3 \mathrm{D}$ authoring and development environment, see http://www.blender.org

${ }^{13} \mathrm{~A}$ generic augmented reality library, see http://www.hitl.washington.edu/artoolkit
} 


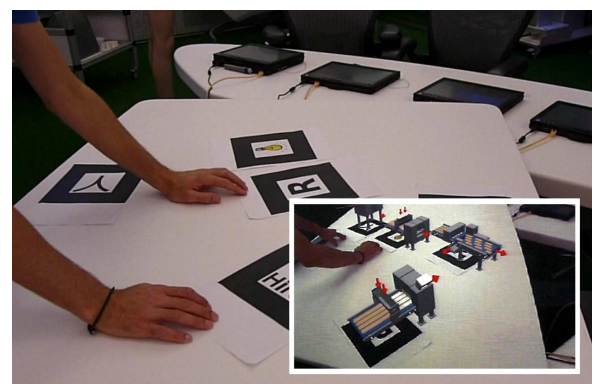

a. The AR Factory Layout demo

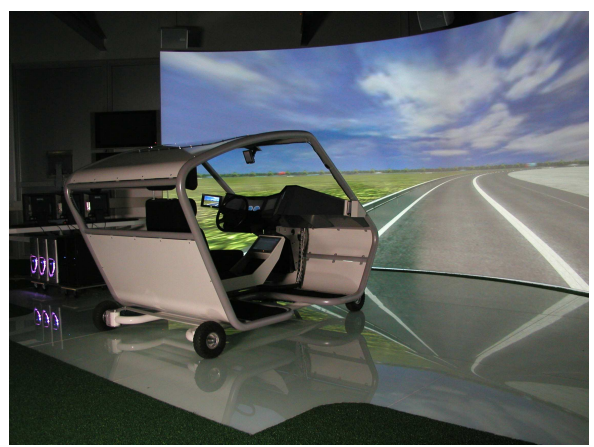

c. The Driving Simulator demo

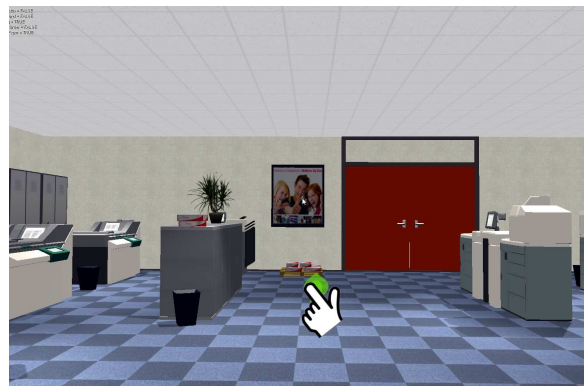

b. The Virtual Usability Lab demo

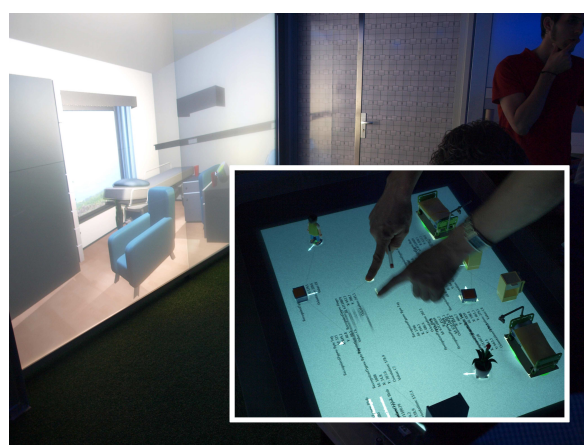

d. The Virtual Playground demo

Figure 2.2 These VR demonstrators were used in a demonstration session to provide the participating designers with a shared understanding of how VR could potentially facilitate design activities.

3. Driving Simulator - An immersive driving simulator available from Tideman et al. (2008), consisting of a physical car frame in front of a large semi-spherical screen, was demonstrated (see figure 2.2c). The driving simulator allows participants to configure their own driving support system (e.g. lane change support, adaptive cruise control, etc.) and immediately experience it in a traffic scenario. In addition to this, a demonstration of motion tracking was provided, which was implemented by putting the driver in an Xsens tracking suit ${ }^{14}$ and showing the output on a screen next to the simulator.

4. Virtual Playground - The fourth demonstrator consisted of a 3D interactive room, again created using the Blender game engine, designed to evaluate lighting and sound effects (e.g. real-time shadows, shading and ambient sounds). The layout of the room can be modified by moving around tangible furniture models on a Surface Table ${ }^{15}$, which was connected to the 3D environment (see figure 2.2d). This provided lighting and sound designers with an intuitive tangible interface to change and review the room layout.

The demonstration session took place in the VR Lab of the University of Twente. The participating industrial partners were represented by at least four participants, including design-

\footnotetext{
${ }^{14} \mathrm{~A}$ 3D motion capture suit, see http://www.xsens.com

${ }^{15}$ Microsoft's surface computing platform, see http://en.wikipedia.org/wiki/Microsoft_PixelSense
} 
ers, managers and researchers. The demonstrators were introduced by explaining how they had been developed, and how they were envisioned to fit the design process of the companies. Participants were invited to try out the demonstrations.

Despite the relatively low-level demonstrators (they were not fully functional as opposed to similar studies such as Cobb et al. (1995)), the variety of applications and technologies triggered detailed discussions between designers and between companies, identifying potential opportunities and bottlenecks in a very early stage of the research, without major investments in application development. Interactions between the participating companies are considered an important aspect of the demonstration session. Even though each demonstrator was designed for a specific design domain, participants were able to reflect on their company-specific application as well as the applications demonstrated for the other companies.

Upon completion of each demonstration, a brief round of discussion was held, and participants were asked to fill out evaluation forms. In the evaluation, participants were asked to assess the use of VR in the PDP; when could it be beneficial, who would be involved in using it, and would it be feasible to facilitate user involvement through VR? Together with observations made by the researcher during the demonstration session, the evaluation of the demonstration session led to the conclusions presented in the next subsection.

\subsubsection{Conclusion}

The VR demonstration session provided the participating companies with an overview of a wide range of VR technologies (i.e. low-end and high-end) and formed a common starting point for discussions about $V R$. The main finding resulting from the session is the distinction between VR applications and VR tools. The VR application describes the concrete design activity in which a particular VR technology is used to facilitate (or enable) the activity. For example, the Virtual Usability Lab uses a 3D walk-through (technology) in an application that facilitates usability evaluations. The tools required to realise this application include the modelling tools for creating the content of the virtual environment, as well as the 3D engine that provides the walkthrough functionality. A clear definition of why and how VR is being used (i.e. the application description) is required for product designers to elicit relevant tool requirements. A related finding was that product designers prefer to realise a specific application themselves, primarily because of time-constraints. Consequently, the tools to realise these applications should meet the skills and requirements of the designers.

Another important finding resulting from the interviews and site visits, is that in the early stages of the PDP, UCD artefacts are generally considered subordinate to the primary purpose of gaining insights in user characteristics, product use and context of use. This is an important difference with (UCD) activities in other stages of the PDP, where the main purpose of design activities is to put knowledge or information into a (representation of) the future product. In other words, early stage UCD tools and methods are primarily mediating between designers and end-users in order to elicit design information, rather than directly helping designers with defining (future) products.

\subsection{Discussion}

This section reflects on the findings presented in the preceding sections, leading to 1) a more profound justification of the research objective and 2) insights in how the objective is to be achieved. 


\subsubsection{Research objective}

As stated in chapter 1 , the primary objective of the current research is "to provide insights in the feasibility of virtual reality as a means to facilitate user centred design tasks in the early stages of a product development process". To justify this objective, an important question to be answered is why VR should be used to facilitate early stage UCD tasks. The answer to this question is provided by the findings of sections $2.1,2.2$ and 2.3.

\section{Facilitating early stage UCD activities}

Section 2.1 showed that UCD activities can take place throughout the PDP. The activities facilitate the elicitation of design information from end-users by presenting, generating or evaluating product concepts. The earlier this information is available to product designers, the more effectively it can be used to (positively) change the direction of the product development. While activities in the advanced stages of the PDP, such as usability evaluations or field tests also inform designers, the information is usually gathered too late to drastically change the ongoing development process. Consequently, the impact of UCD activities on the PDP is most significant in the early stages of the PDP, when the design information elicited from end-users can still be used to adjust the course of the development process.

One challenge that is particularly relevant for the early stage development of complex and/or interactive products, is to provide end-users with a complete and integrated view of the future product and its future use context. Current UCD techniques, such as a mockup of the user interface, a 3D model of the exterior design or an animation of functional behaviour, rely on artefacts that typically convey only a part of this information. VR potentially enables a multi-disciplinary development team to integrate multiple product aspects in one virtual model. Then, after introducing end-users to an overview of the virtual model, designers and end-users can 'zoom in' on specific aspects of the model.

Up to recently VR technologies could not be considered 'fit' for use in early design stages as the technology was relatively unknown, expensive and difficult to deploy. The current state of VR technology, however, has reached a level where technology and content (e.g. digital assets) have become widely available. Digital models can easily be created, stored and shared. Advanced technologies such as gesture recognition, multi-touch input and high-end visualisation are available off the shelf. It is argued that this allows the use of VR (and its contents) to be as 'disposable' as sketches and foam models are now. In other words, the technology has evolved to a level where it better fits the nature of UCD and conceptual design. This argument is supported by the findings of the field study presented in section 2.3. While designers initially perceived VR technologies to be difficult to use and not really fit for early stage design activities, this perception changed after demonstrating the current state (and variety) of VR technologies and applications thereof. Practitioners acknowledged the potential added value to early stages design activities, and indicated the need for (and feasibility of) means (i.e. tools) to realise these VR applications themselves. This last point stresses the need for the current research to not only identify advantageous applications of VR for UCD, but also determine the boundary conditions for realising these applications in design practice.

\section{Challenges of deploying VR in the early stages of the PDP}

As explained in section 2.2, existing applications of VR in the PDP mainly take place in the engineering and manufacturing stages because of the traditional relations to CAD. While some 
applications also address the conceptual design stages (e.g. virtual prototyping), several aspects make UCD fundamentally different from other conceptual design tasks.

1. Lack of structure - Koen et al. (2001) characterise the front-end of the PDP as unstructured; in contrast to subsequent stages of the PDP, there is neither a concrete definition of what happens in the fuzzy front-end, nor a consistent outcome or deliverable. Nobelius and Trygg (2002) state that while it might be desirable to structure (in order to optimise) the front-end, it is not likely to be achieved through one concrete structure or approach.

2. Lack of design information - As UCD activities are often used in the ideation phase of a project, there are no concrete specification of dimensions or material selection, and sometimes not even a fully defined set of product functions. Unlike VR applications in advanced stages of the PDP, VR applications in the early stages can not rely on the availability of detailed CAD models (nor are they considered appropriate for these tasks, as explained in section 2.2.4).

3. Time pressure - There is an increasing need for more efficiency (shorter life cycles) and flexibility (technological and environmental developments) in product development in general, and for conceptual design in particular, given the significant impact of this phase on the total outcome of the development process.

4. Non-expert stakeholders - When involving end-users in design activities there generally is no or limited time available for training. Furthermore, end-users are not only untrained (in terms of design activities), but also prone to being distracted by tools or artefacts that are not directly related to the product. This means that any VR application used in UCD should be easy to use for untrained users, and not distract from the original purpose of the UCD activity itself.

5. Mediation through design artefacts - Existing VR design support tools are based on (or extend) relatively detailed CAD models. The model mediates between its operator (the designer or an end-user) and the final product. In UCD activities however, design artefacts typically mediate between the end-user and the designer (as found in section 2.1). This is a fundamentally different way of interpreting, and interacting with, design artefacts.

\subsubsection{Towards an approach}

In preparation of an approach for the current research, this section reviews related work in which similar objectives were pursued. Two types of approaches are discussed, namely a technology driven specific approach, where a specific technology is implemented in a specific design case, and an application driven generic approach, where designers are provided with means to select an appropriate form of VR for their particular design case. The differences between the aforementioned specific and generic approaches towards providing design practice with useful VR applications are illustrated in figure 2.3.

\section{Specific vs. generic approaches}

The majority of work in the field of VR uses the technology driven approach, as illustrated by the large number of examples of specific VR applications in section 2.2. The technology driven approach 'pushes' a specific technology to design practice, as illustrated in figure 2.3a. As such applications are typically developed for a specific context or purpose, a risk of this approach is 


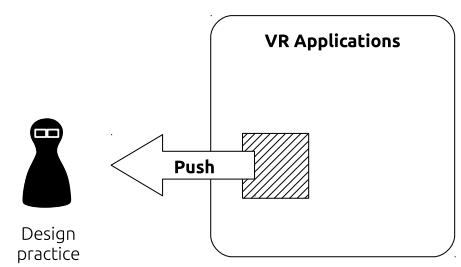

a. VR applications are 'pushed' into design practice

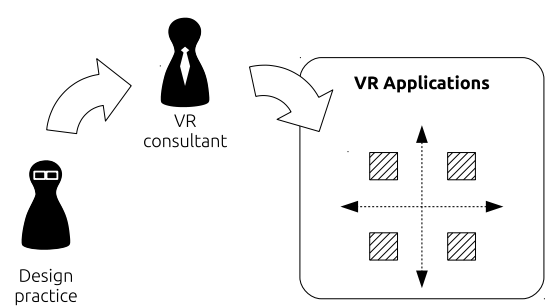

b. Mediated negotiation between practice and technology

Figure 2.3 Different approaches to provide design practice with advantageous VR applications.

that the application will not work as intended in different design contexts. This is particularly likely to occur in the unstructured early stages of the PDP. Furthermore, as Miedema (2010) states; "academics cannot and will not develop dedicated applications for all niches". In addition, from the perspective of UCD and VR, these types of applications are less likely to be adopted for early stage UCD tasks, because designers are not likely to be aware of (domain) specific applications, and because designers like to have some flexibility in how they use a specific tool, rather than being told how and when to use it.

Miedema (2010) therefore provides a consultancy framework based on which product designers are given advice on what type of synthetic environment would be beneficial for a specific product development activity. Figure 2.3b illustrates this generic approach. The consultancy procedure consists of 9 successive stages, guiding the designers through a series of (among others) exploratory activities, a requirement specification phase, an effect analysis and the presentation of a final proposal. This consultancy framework is an answer to the lack of generic knowledge about applying synthetic environments and makes good use of the specific knowledge that is typically provided by literature.

\section{Limitations of existing work}

A potential drawback of the application driven approach is that the selection of an appropriate VR technology requires external expertise, for instance provided by a VR consultant. While this can be an apt solution for design stages where time is less constrained, it was found in the field study that designers prefer to remain in charge of not only selecting the appropriate design tool, but also of deploying it in the design process. Interestingly, Miedema's decision to rely on external expertise was based on a field study similar to the one presented in this chapter;

"The knowledge required to estimate the pros and cons of applying Synthetic Environments in a particular project should rest with a trained colleague or an external consultant. [...] specific hardware and software should be accessible at an external facility to make it more interesting for small and medium sized enterprises".

The fact that the current work identified the exact opposite of this can be explained by three factors. Firstly, it is argued that this statement depends on how VR (or synthetic environments) is initially presented to product designers. Assuming a generally limited knowledge of VR technologies, it can be expected that people who are confronted with relatively high-end technologies (in the mentioned work, one live demonstrator using an FCS Haptic Master was 
used) prefer to rely on external resources for realising these applications. The field study in the current research demonstrated a wider range of applications and technologies and featured somewhat more low-end VR technologies such as desktop VR and augmented reality. A second factor that contributes to this finding is that the focus of the current research is on UCD activities in the early stages of the PDP, while Miedema addresses the early stages in general. A third factor is that nowadays technologies have become much more easily available for consumers (and designers), as also discussed in section 2.2.3. This in turn may have affected the designer's attitude with respect to VR technologies; awareness of for instance the Wii or the Kinect may have reduced the perceived difficulty.

\subsubsection{Conclusion}

The discussion presented in section 2.4.1 leads to three assumptions that are to be verified in the present research.

1. VR technologies facilitate UCD tasks in the early stages of the PDP by enabling product designers and end-users to view the future product in its expected use context.

2. Low-end off the shelf VR techniques provide sufficient means for the realisation of VR applications that facilitate early stage UCD activities.

3. The primary bottleneck for design practitioners to realise these applications lies in the usability and accessibility of the tools needed to to realise these applications.

Section 2.4.2 showed two common approaches towards providing design practice with advantageous applications, namely a technology driven approach and an application driven approach. Technology driven approaches fail to negotiate between the needs of design practice and the opportunities offered by VR technologies. Application driven approaches provide negotiation, but lack an established set of applications specifically designed to facilitate UCD tasks. Furthermore, neither the specific nor the generic approach covers the realisation of VR applications through appropriate tools. In the field study it was shown that because of the flexibility, lack of time and lack of structure of the design stages in which these activities take place, product designers prefer to realise, adapt and deploy the applications themselves.

The next chapter therefore presents a new approach for 1 ) identifying applications that specifically facilitate UCD activities, and 2) identifying the boundary conditions for the realisation of these applications. 


\section{$3 \circ$ Approach}

As introduced in chapter 1 , the objective of this research is to provide insights in the feasibility of VR as a means to facilitate UCD tasks in the early stages of a PDP. Chapter 2 concluded that a new approach is needed to 1) identify applications that specifically facilitate UCD activities, and 2) identify the boundary conditions for the realisation of these applications. This new approach is presented in the first section of this chapter. Sections 3.2 and 3.3 outline the two methods that support the execution of the approach.

\subsection{Overview}

This section elaborates on the key characteristics of the research approach, and presents its main structure and anticipated output.

\subsubsection{Key characteristics}

The definition of the approach is based on three key characteristics. During the VR demonstration (see chapter 2 section 2.3) it was found that relevant boundary conditions for the realisation of VR applications can be elicited from product designers by providing them with a clear description of a relevant application. The application provides a frame of reference that helps assess the relevance of a (technical) requirement and provide a specification of a requirement (e.g. the required level of realism in a specific application). This consideration forms the basis of the approach and supporting methods presented in this chapter: requirements regarding realisation need to be determined within the context of a specific VR application.

Crosier et al. (2002) faced a similar challenge during the development of a VR supported educational application. A user (teacher) centred approach is defined consisting of three phases: a selection phase, a design \& development phase and an evaluaton phase. Of particular interest is the design \& development phase, as it prescribes design iterations of the VR application that include teachers as evaluators of the prototype application. The researchers used low fidelity techniques such as storyboards to let teachers describe their anticipations with respect to the VR application. These means help identify and discuss the so called contextual considerations that might otherwise be ignored. As the authors conclude: "observing students using the software and gathering their opinions of it will ensure that in addition to the educational goals being satisfied, the software is useful, enjoyable and usable by students". The user centred elements of this work will be adopted in the current research; the development of VR applications for UCD as well as the identification of tools to realise them should actively involve product designers. A final aspect affecting the definition of the research approach is the need for generic insights regarding both the VR applications and the tools that (together) facilitate UCD tasks. The first 
two approach characteristics mainly concern the definition of case specific VR solutions, similar to how specific approaches (as discussed in section 2.4.2 of chapter 2) traditionally develop VR applications. Conform the objective of the current research, the approach will include a generalisation step in which the case specific insights are translated to generic insights regarding VR applications and VR tools. The definition of an appropriate framework for structuring these insights is also part of the approach.

\subsubsection{Structure}

The research approach consists of four phases, as illustrated in figure 3.1.

1. Specification phase - The specification phase involves three industrial case studies. Each case study features a different design context not only in terms of the type of product that is being developed, but also the type of development (e.g. radical innovation vs. incremental innovation) and the level of experience with user involvement in the early stages of the PDP. For each company a VR application will be identified, developed and deployed. Subsequently, the realisation of the application will be investigated by providing the designers with tools to create the application themselves. The method used for conducting the case studies is further elaborated in section 3.2.

2. Generalisation phase - The case specific solutions will be placed in new design contexts, namely those of the other two companies. By reviewing the solution from these new perspectives, we learn whether or not a solution for design context $A$ also works for design contexts $B$ and $C$; are the supported UCD tasks also relevant for companies $B$ and $C$, and are the selected $V R$ technologies appropriate for deployment within companies $B$ and C? The method used to implement this step is presented in section 3.3.

3. Externalisation of insights - Generalisation of the case specific insights results in knowledge about why, when and how VR can be used effectively to facilitate UCD tasks in various design contexts. This knowledge needs to be structured and externalised in order for design practice to benefit from it. This part of the approach provides an appropriate means for externalising the knowledge. Section 3.1.3 provides a first step towards structuring the results.

4. Reflection on approach - The reflection phase reviews the generic insights presented in the externalisation phase, as well as the methods used in phases 1 and 2 .

(a) A reflection on the resulting insights - A critical review of the insights gathered from the case studies and the generalisation phases.

(b) A reflection on the research methods used in (1) and (2) - Based on the proceedings of the research, the methods used in phases 1 and 2 can be optimised and possibly applied in other design research.

The company specific case studies are conducted in series, meaning that the three industrial partners are involved one after another. Between each case study, the cross-company evaluations take place. Each case study takes about six months, providing sufficient time for the identification, development and evaluation of an extensive VR solution. The cross-company evaluations are one day events that take place between the three case studies, as also illustrated in figure 3.1 . 


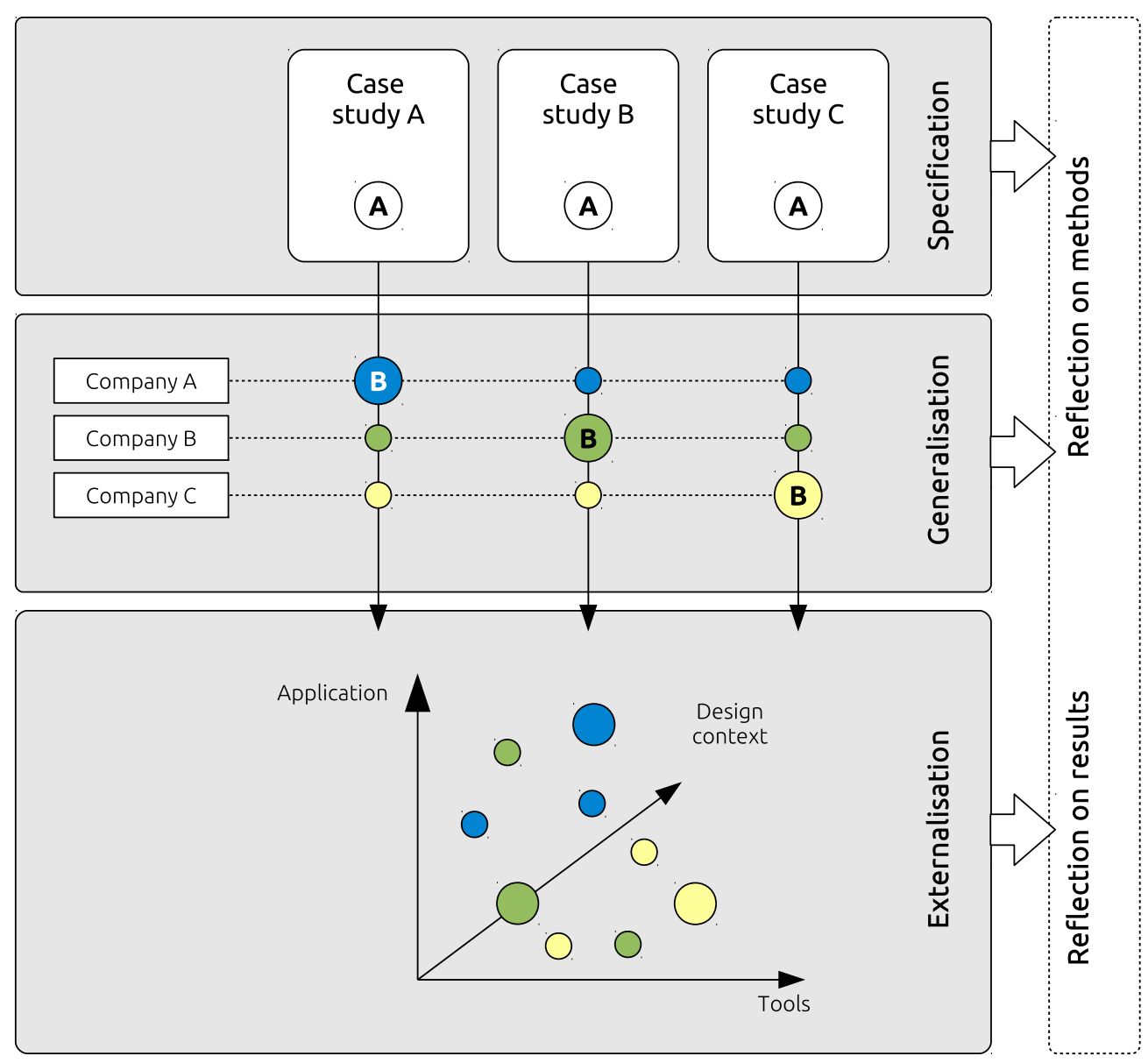

Figure 3.1 This diagram illustrates the phases of the research approach. Labels $A$ and $B$ represent the two methods that were designed to support the approach. Method A (see section 3.2) is used in all three case studies, and prescribes the steps taken to identify, develop and evaluate VR applications and tools for each company. Method B (see section 3.3) prescribes steps needed to evaluate the case study results in the contexts of the other companies involved. 


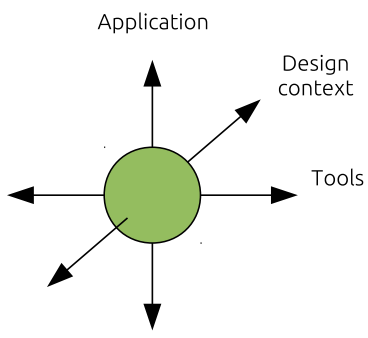

a. Generalisation.

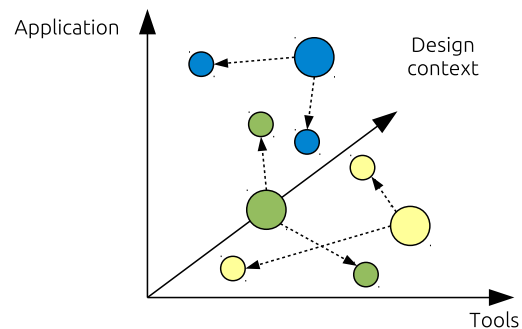

b. Anticipated framework.

Figure 3.2 The generalisation phase aims to investigate the flexibility of the VR solution's tool and context dimension. The output of the generalisation phase is structured in the above framework.

\subsubsection{Output}

The research approach leads to three layers of results.

Firstly, the specification phase provides each industrial partner with practical experience in using VR to facilitate UCD activities. While the primary aim of this phase is to provide the researcher with practical insights, the companies involved also gain first-hand experience with an unfamiliar but potentially valuable technology.

Secondly, the iterative execution and improvement of the method used to complete the specification phase provides the foundations of a solid method for identifying, developing and deploying advantageous applications of VR in the PDP. This output is expected to be useful for design research as it illustrates the application of several design techniques in a practical case study as well as design practice, where it can be used as a technique to explore benefits of potentially interesting technologies.

The primary output with respect to the current research however consists of the knowledge regarding the facilitation of UCD through $V R$, gained during the generalisation phase. This output will be structured in a framework described by three dimensions, namely context, application and tool, as illustrated in figure 3.2.

1. Design Context - The design context refers to the 'design circumstances'; the type of product, the designers involved and the type of process in which it is being developed all have influence on selecting a particular design task and support tool. For instance, radical innovation requires a different approach to design than incremental innovation, and a digital product is likely to benefit from different types of support than a tangible product.

2. Application - This dimension describes the purpose that a designer has when conducting a certain activity. During the generalisation phase the application developed for one specific design context will be translated to other design contexts. While the content and technical solution for the application may change, the core application should remain constant in order to describe its flexibility across design domains.

3. Tools - The tool dimension describes the tools and technologies used to optimally support the realisation of a VR application in a given design context. 
The three concrete case studies form points within the three-dimensional space spanned by these dimensions, describing a solution of VR for a particular UCD activity, implemented in a particular PDP. In the generalisation phase, the flexibility of each solution with respect to the three dimensions will be assessed (see figure 3.2a), resulting in a total of nine 'points' that represent the scope of the investigated VR solutions, as illustrated in figure $3.2 \mathrm{~b}$.

\subsection{Case study method}

This section presents the development of the method that is used to conduct the company specific case studies. The method structures the development of a VR solution for a given design context (provided by the industrial partner involved in the case study). While in essence the method describes a software development project, the method also involves aspects of existing participatory design techniques.

As explained in section 3.1.1, requirements regarding the realisation of VR solutions should be investigated within the context of a specific VR application. Consequently, the philosophy behind the case study method is to first describe the use of VR in a product design context, and to then create (or select) the tools that fit the realisation of this application.

The method consists of the following steps.

1. Explorative workshop - A participatory design workshop involving a group of company participants is organised to identify advantageous applications of VR, and select one application for further use in the case study.

2. Prototype development - A functional prototype of the selected application is developed by the researcher.

3. Validation of application - The functional application prototype is deployed in a test session to evaluate and validate the anticipated advantages of the application.

4. Tool selection - Following a successful identification of a VR application, appropriate tools are selected or developed by the researcher.

5. Tool evaluation - The selected tools are presented to and evaluated by company participants in order to assess their usability in a specific design context.

The following subsections further elaborate on these steps.

\subsubsection{Explorative workshop}

The first step in the method aims to identify a useful application of VR within the early stages of the design process of the participating company. This requires collective in-depth knowledge of the company's design process and knowledge of the available VR technologies. A workshop format is used in which company participants provide the former, and the researcher provides the latter. To make sure that both parties to understand each other, visual storyboards form a common language. The use of visual storyboards is inspired by various participatory design methods such as Inspiration Cards (Halskov and Dalsgard (2006)), Pivots (Urnes et al. (2002)) and the Future Technology Workshop (Vavoula et al. (2002)). 


\section{Structure}

The workshop is a three hour session involving a multidisciplinary group of about 10 participants. The workshop is structured as follows:

1. Introductory presentation - This presentation explains that the purpose of the session is for the participants to generate potential applications of VR in the PDP of the company. The presentation also introduces $V R$, by presenting several examples of technologies that together span the real-virtual continuum presented in chapter 2 .

2. Presentation of example storyboards - To illustrate the concept of storyboards, the researcher presents four animated storyboards that were prepared earlier. The storyboards visualise different applications of VR in the PDP of the company. The ideas for these storyboards are derived from interviews and observations.

3. Individual storyboard - After showing the example storyboards, the animation is broken down into key frames; paper frames that depict key events in the storyboard. Participants are asked to use these frames to generate their own storyboards by

(a) modifying the order of the frames of one of the example storyboards,

(b) merging example storyboards,

(c) adding customised (hand drawn) frames, and

(d) adding technology frames (prepared by the researcher), depicting the use of specific VR technologies.

The resulting individual storyboards are presented to the group. Participants are encouraged to review the applications critically by explaining when, how and why VR is applied (visualised by the technology frames).

4. Group storyboard - After discussing the individual storyboards, groups of three to four participants are formed based on similarities in storyboard themes. The groups are asked to discuss, compare and merge their storyboards into a group storyboard. The group storyboards are further detailed by letting the participants think about the following questions with respect to the application that is visualised in a particular storyboard:

(a) Who are involved in this application?

(b) When in the design process does the application occur?

(c) What is the primary purpose of the application?

(d) Which resources are required for the application?

In addition to providing detailed documentation for the researcher, these questions also trigger more in depth discussions about the application.

5. Wrap-up - During the wrap-up, group storyboards are presented to all participants. The aim of these presentations is to share and discuss the group storyboards, and to reach consensus about which of the group storyboard presents the most interesting storyboard for further use in the case study. 


\subsubsection{Prototype development}

The prototype development step aims to provide the participating designers with a sufficiently functional prototype of the application identified in the explorative workshop. Although software development is not the focus of this research, it is considered necessary to provide the participants with a functional VR application in order to elicit relevant insights and feedback.

The following guidelines are used to structure the development:

1. The development should be user-centred; since product designers are considered the endusers of the VR application, they should be involved in various iterations of the development.

2. As concluded in section 2.4.3, it is expected that low-end off the shelf technologies provide sufficient means for facilitating early stage UCD activities. Consequently, for the development of a functional prototype the researcher will make use of this range of VR technologies as much as possible.

3. Additional refinements of the application description are needed to achieve the 'core function' of the anticipated application. This means that the researcher, together with participating designers, may need to go through one or two rounds of refinements and discussions after the explorative workshop.

4. The primary aim of the prototype is to validate the VR application. The development of the prototype should therefore not be constrained by external dependencies, such as compatibility with CAD tools or databases used by a company, or compatibility with hardware available within a company. In a prototype these issues can be fixed for instance by manually importing external models (if necessary). After validating the application it can be determined whether or not e.g. CAD compatibility or hardware compatibility is still relevant, and if so, how it can be implemented (this is considered a tool aspect).

An important aspect of prototype development is the consideration of application parameters. Even after establishing a clear application based on the results of the exploration workshop, it can be expected that product designers are not sure about certain application parameters, such as the required level of realism, the preferred form of interaction or the preferred form of virtuality. By including these parameters as variables in the prototype, participating designers can experience the effects of each parameter. For example, when designers are not sure about the required level of visual realism, the prototype could provide two or three configurable levels of realism.

\subsubsection{Application validation}

The third step in the case study method is to verify the anticipated value of the VR application by deploying the application prototype in a test session. During the test session, the VR application is used in the way it was visualised in the initial storyboard, allowing the researcher and the participating designers to verify whether the selected VR technologies sufficiently facilitate the UCD activity. When selected VR technologies do not properly support UCD activities, the use of alternative (or possibly more high-end) technologies can be considered. The validation focuses on obtaining qualitative as well as quantitative results. Quantiative results, obtained through post-session evaluation forms, will be used to verify the qualitative results, which will 
be gathered by analysing video recordings of the validation sessions and the results of the test case.

In addition to validating the application itself, the session also investigates the effects of the different application parameters that were identified during prototype development. The test session enables participants to explore these parameters, which are difficult to determine without actually experiencing the application. Examples of such parameters are the required level of realism of a virtual environment, or the desired type of interaction with a virtual environment. By experiencing these parameters in the application prototype, designers are able to determine a desired or required implementation. During this assessment the researcher should make sure that participants treat the application parameter as a trade-off rather than an unconstrained variable. The trade-off primarily regards the tools that are required to realise the desired application parameter. In the example of visual realism, the need for a highly realistic visualisation may in turn require more advanced tools (e.g. professional 3D modelling tools), additional resources (e.g. high resolution images) and additional realisation efforts (e.g. 3D modelling). The experience gained during the development of the prototype can be used to help participants make valid assessments.

\subsubsection{Tool selection}

The objective of the tool selection step is to identify tools that enable designers to realise the VR application as developed in the preceding steps. While in some cases the tools used during the prototype development step can also be appropriate for use in design practice, in general it can be expected that the tools meant for deployment in design practice have different requirements with regard to usability, functionality and flexibility. For example, the researcher can use a versatile and complex Game Engine to develop a 3D walk-through application prototype, while in practice designers are looking for a drag-and-drop solution to quickly compose a similar application.

The tool selection involves two types of tools:

1. Preparation tools - Preparation tools are used prior to the execution of the application, and support the creation or acquisition of the virtual objects, context and behaviour required for the VR application.

2. Execution tools - Execution tools provide functionality required during the use of the application. For example, an AR application requires tools to recognise AR markers and connect these markers to 3D objects.

Contrary to the preceding steps, this activity is carried out by the researcher alone, who now fulfils the role of 'VR expert'. After a decomposition of the VR application into the core tasks that are required to realise the application (e.g. 'create a 3D office environment' or 'define interactive behaviour'), tools are selected that support these tasks. The selection of appropriate tools and technologies is constrained by

1. contextual factors derived from company characteristics, such as the available skills, time budgets and people (gathered during the exploration workshop and further refined during the application validation) ,

2. contextual factors imposed by the application, such as the required compatibility with existing tools (e.g. CAD systems) or the compatibility with software and hardware platforms available in the company, and 

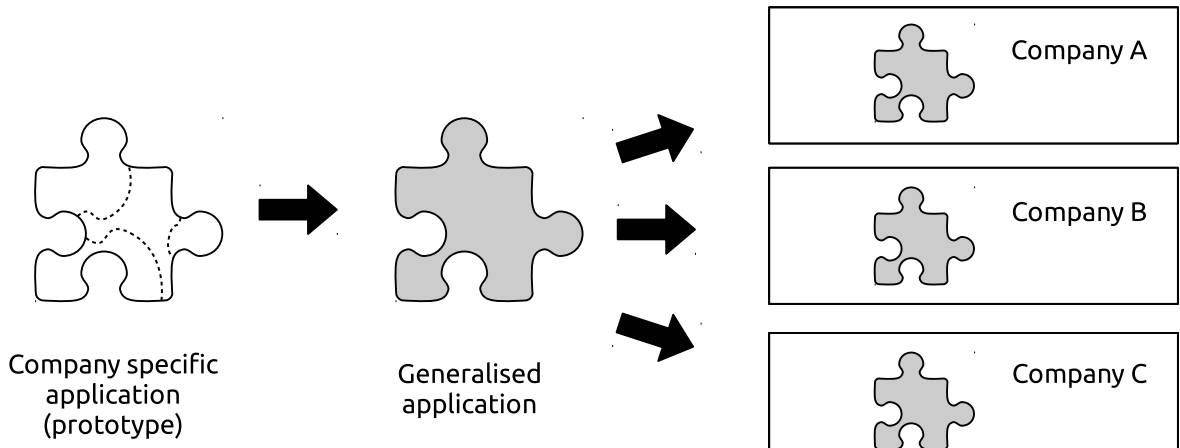

Generalised application

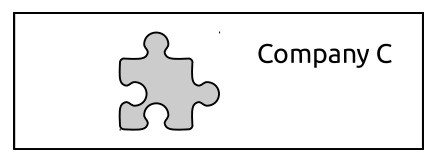

Figure 3.3 Visual outline of the cross-company evaluation session.

3. the preference for low-end and off-the-shelf VR technologies as defined earlier, indicating that off-the-shelf tools and technologies should be used as much as possible.

\subsubsection{Tool evaluation}

The final step in the case study method is to evaluate the effectiveness and usability of the tools that were selected by the researcher. Effectiveness, in this context, refers to whether or not the tools enable designers to realise the desired application, while usability refers to how easy it is for them to do so. Depending on the selected tools, this evaluation can consist of a workshop in which the tools are used by the designers directly (if the selected tools are directly available to the researcher and/or company), or it can be based on a presentation of the proposed tools (if the selected tools are not directly available or do not exist yet).

\subsection{Generalisation method}

The generalisation method structures the translation of the company specific results into generic insights regarding the VR application and its supporting tools. As shown in figure 3.1, this translation takes place after each industrial case study, and is referred to as a Cross Company Evaluation (CCE). The CCE sessions consist of collaborative meetings in which all the participating companies are involved. Figure 3.3 visually outlines the purpose of the CCE. The jigsaw-piece represents the VR solution that was created during the case study. This solution is translated (on a more generic level, indicated by the hatched piece) to other design domains and companies. 


\subsubsection{Session Structure}

The session consists of the following steps.

1. Case study review - The results of the case study are presented to the participants. The presentation outlines the main events of the case study, such as workshops, demonstrations and evaluations. The presentation is co-hosted by company representatives who give feedback on how they experienced the case study and how the results affected their work.

2. Demonstration of results - The application and tool selection resulting from the case study are presented to the participating companies. Depending on the state of the application prototype and the availability of supporting tools, participants are invited to engage in a use case and experience the application first-hand.

3. Discussion - The participating companies (in groups) are given the assignment to translate the presented results into something useful to their company. This requires them to describe how the application would be used (i.e. its purpose) and who would be developing and/or using it.

The discussion facilitates an important step of the generalisation as it involves the translation of the case specific solution into a solution that is relevant to the other companies. The translation contains two variables that can potentially be changed, namely the VR application and the supporting tools. However, as explained in section 3.1.3, in order to assess the flexibility of the application that was developed for a specific design context, the core application should remain unchanged as much as possible. Allowing participants to change both the application and the tools would potentially result in three completely new and different VR solutions in each CCE, instead of deeper insights regarding a specific combination.

To maintain this focus, the following discussion structure is used

1. Split the application into 1) an abstract core that remains constant and 2) concrete content that needs to be tailored to a specific company

2. Change the content to suit the design domain of a specific company

3. Assess the added value of this application to the company's PDP

(a) Discuss application parameters

(b) Discuss boundary conditions for integration in the PDP

4. Discuss the effectiveness and usability of the supporting tools

\subsubsection{Output}

The CCE sessions form an important part in the research approach. Firstly, it is an opportunity for the companies that are not directly involved in a case study (the companies are involved one by one) to get first-hand experience with the VR applications developed in the case study, which contributes to their commitment and sense of involvement in the project, and broadens their perspective on potential VR opportunities for their own practice. Secondly, it is an opportunity for the researcher to gain insight into the usefulness of the developed VR application across various design contexts, and the effectiveness and usability of the supporting tools. 
The concrete translations of case study results to other companies that were derived during the CCE's provide access to these insights; it enables the researcher to ask practitioners why they do or do not acknowledge the benefits of a specific application or why tools do or do not integrate with their PDP. These deeper insights can be gathered during the sessions through discussions, and also from post-session data such as video and audio recordings of the proceedings, or written evaluation forms.

Although the application core should remain unchanged as much as possible, the CCE session may evoke participants to describe new applications (i.e. different from the application resulting from the case study), or misinterpret or re-interpret the core functions of the application so that the definition of a new application is justified. When interpreting the results of the CCE sessions, the researcher should constantly consider whether the insights are still referring to the intended application, or actually describing a new one.

\subsection{Implementation}

The methods presented in this chapter have been implemented in three industrial case studies, and their respective cross-company evaluations. Figure 3.4 illustrates the coverage of the results in subsequent chapters.

The proceedings of the case studies are presented in chapters 4,5 and 6 .

1. Case study 1 covers the development of the Virtual Printshop for the industrial partner involved in the design and development of professional office machinery. The Virtual Printshop allows end-users and designers to operate and evaluate candidate printer concepts in an interactive 3D virtual environment.

2. Case study 2 involves the development of Virtual Personas that enable the truck design company to act out virtual scenarios in a very early stage of the design process. The virtual personas used in these scenarios represent user groups, such as 'older drivers' or 'tech-savy drivers', and can be used to review a new product concept from these specific points of view.

3. Case study 3 introduces a Virtual Annotation application that was developed for the industrial partner involved in the development of food processing solutions. The application facilitates the creation, review and annotation of virtual candidate designs in multidisciplinary groups.

The results of the cross-company evaluations as well as a detailed discussion of the generalised insights derived from them are presented in chapter 7 . 


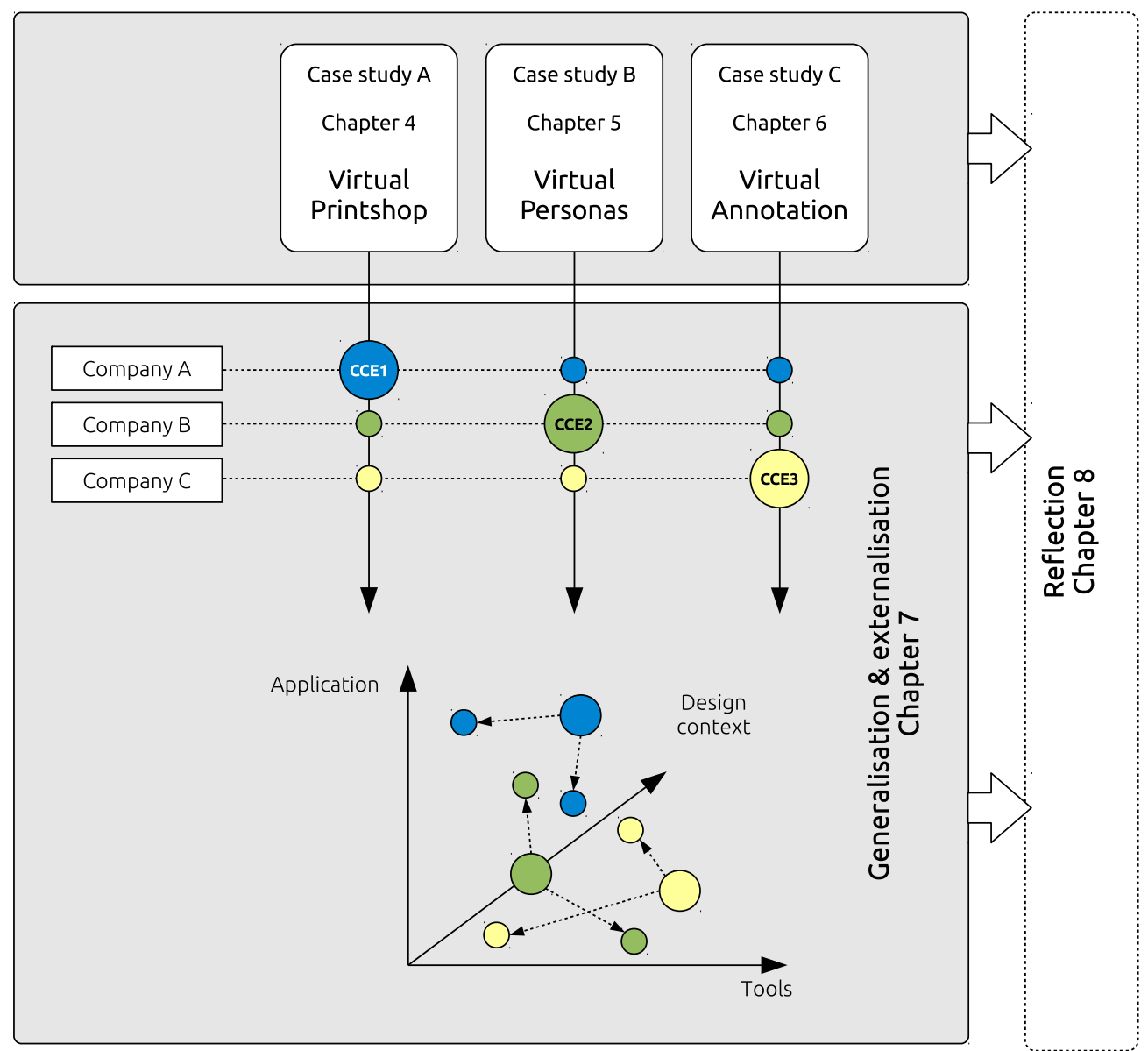

Figure 3.4 The results of the approach implementation are reported in the next four chapters. 


\section{$4 \quad$ Case Study 1}

The Virtual Printshop introduced in this chapter aims to facilitate product evaluations in the early stages of the development process by providing a more realistic test environment. In usability evaluations the product's use context can play an important role in triggering feedback from either end-users involved in the evaluation or from designers themselves. The Virtual Printshop provides a realistic and interactive virtual environment in which virtual product models can be experienced, for instance by acting out workflows or specific use scenarios. The identification, development and deployment of this VR application took place according to the specification method presented in chapter 3 .

\subsection{Introduction}

The company involved in this case study is a multinational manufacturer of office printing and copying systems, high-speed digital production printers and wide format printing systems. The company's design department includes interaction designers, product designers, visual designers, usability engineers and software prototypers. The department is primarily involved in the design of user interfaces and user-product interactions. While the end-users of the printers are typically trained operators, designing a good user interface is challenging because of the technical complexity of the machines, and because of the various use contexts in which the products are used (e.g. universities, small offices or professional printshops).

During the field study (see section 2.3) it was found that the company's PDP already featured several UCD activities in the early stages, including site visits, wizard-of-oz evaluations with mockups, the use of personas and usability evaluations with functional prototypes. Nevertheless, the design department is interested in finding additional tools and methods for actively involving their end-users in the design and evaluation of new printers.

\section{Chapter outline}

The activities defined in the specification method have been divided into an exploration phase, a development phase and a deployment phase. The exploration phase, described in section 4.2, further investigates how VR applications can potentially support the company's PDP. In section 4.3 a VR application prototype is developed and validated in practice. The deployment phase, outlined in section 4.4, describes how the application can be realised within the company's PDP. 


\subsection{Exploration}

The Exploration phase covers the exploration of potentially interesting applications of VR for the company, eventually leading to the definition of the Virtual Printshop. The following subsections outline the ideation and selection process that resulted in this definition.

\subsubsection{Kick-off}

The kick-off meeting is a preliminary meeting in which the researcher outlines and clarifies the case study approach to the participating practitioners and in which the initial focus of the case study is established.

To determine the case study focus the participants are asked to describe challenging or problematic events and activities in their PDP. The results of this brainstorm session are summarised as follows:

1. Internal Concept Reviews - The internal concept review is a meeting between designers with a shared background, such as visual design or interaction design. The review aims to discuss a new idea with experts in the field before showing it to a larger group. The primary challenge of these meetings is for experts to 'think ahead' and assess how their specific component relates to the product as a whole.

2. Group Concept Reviews - Group concept reviews are used to present a new concept or idea to a large multi-disciplinary group of designers or project members. People involved in this meeting can share their opinions or indicate specific problems (e.g. a software developer could indicate that a new interaction concept is impossible to make). The primary challenge in these meetings is to present a complex product to a multidisciplinary group of stakeholders, and to allow each stakeholder to access detailed information regarding multiple disciplines in order to make reliable assessments.

3. External Usability Tests - Usability tests validate assumptions about the usability of a product. There are various ways of doing usability tests, mainly determined by the stage of the design project; in early stages it is quite common to do quick tests (without actual users), while in a later stage, when a fully functional prototype is available, a lot of different usability topics are investigated simultaneously. The primary challenge of usability evaluations is to provide evaluation participants with a prototype that elicits reliable and relevant feedback.

4. DSI Debrief - DSI (Document Scene Investigation, a form of contextual inquiry) debrief sessions are used to extract knowledge and information from the designer that conducted the contextual inquiry. The primary challenge in DSI debriefs is that the information should be transferred to and stored in the design team as efficiently and effectively as possible, to make sure that no relevant information is lost.

These design activities depict very specific activities and were supported by several anecdotes during the brainstorm. However, they also show several recurring themes that can be used as leads towards potential VR applications in the explorative workshop. For example, activities 1 and 2 describe problems related to the multidisciplinary nature of the company's design teams. Activities 3 and 4 address the relevance of the use context, when evaluating the usability of a product and when conducting contextual inquiries respectively. The design activities and their 


\begin{tabular}{ll}
\hline Activity & VR Application \\
\hline Internal concept reviews & $\begin{array}{l}\text { An early stage virtual collaboration application that allows de- } \\
\text { signers to merge low-fidelity sketches with high-fidelity models of } \\
\text { existing products. }\end{array}$ \\
Group concept reviews & $\begin{array}{l}\text { A holographic table that facilitates multidisciplinary design review } \\
\text { meetings by showing an interactive 3D model of the product in } \\
\text { its current state of development. }\end{array}$ \\
Usability tests & $\begin{array}{l}\text { A mobile augmented reality application that allows clients to eval- } \\
\text { uate new printer concepts in their own use environment. }\end{array}$ \\
DSI debrief & $\begin{array}{l}\text { An information capture \& documentation application that allows } \\
\text { designers to capture a client's use environment and effectively } \\
\text { transfer this information to the design team. }\end{array}$ \\
\hline
\end{tabular}

Table 4.1 VR applications proposed for design activities

underlying themes have been used to create proposals for applying VR to facilitate these activities. The next subsection introduces these proposals and describes their role in the explorative workshop.

\subsubsection{VR exploration workshop}

As explained in chapter 3, the exploration workshop uses visual storyboards to let designers create their own representations of desired VR applications. In the first case study the workshop involved twelve participants, including two usability engineers, three interaction designers, two visual designers, two digital prototypers, a project manager, a software engineer and a product designer. The workshop took place in a meeting room at the company's premises, and took about three hours.

\section{Preparation}

To provide participants with a starting point for creating storyboards, four animated storyboards were prepared based on the design activities identified during the kick-off meeting. For each design activity a supporting VR application is proposed by the researcher and presented in the storyboards, as listed in table 4.1. The animated storyboards illustrate the use of these applications in the design context, for instance by showing a designer using an augmented reality tool (figure 4.1a), or by showing a design meeting being supported by a virtual collaboration tool (figure 4.1b).

The VR applications proposed in the storyboards serve as inspiration and starting point for further discussion of opportunities for VR in the PDP. Consequently, while it can be argued that the feasibility of some of the technologies used in the storyboards (e.g. the holographic table) is quite low, it is considered more important to show a wide range of technologies (e.g. augmented reality, holography, 3D capturing and desktop VR) rather than focus on feasibility in this stage of the case study.

The explorative and inspirational nature of the storyboards also justifies the low fidelity of the animations. The animations are recorded using the stop-motion technique, resulting in a particularly choppy and crude animation. Furthermore, the use of toy actors and paper props contributes to the experimental and unfinished nature of the storyboards, giving workshop 


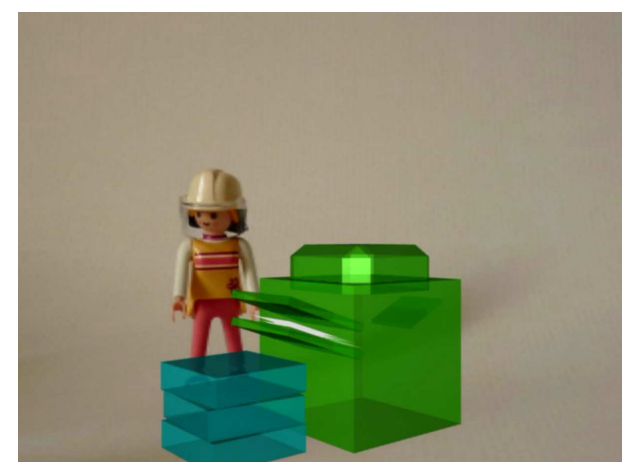

a. A designer using augmented reality to evaluate a virtual printer concept.

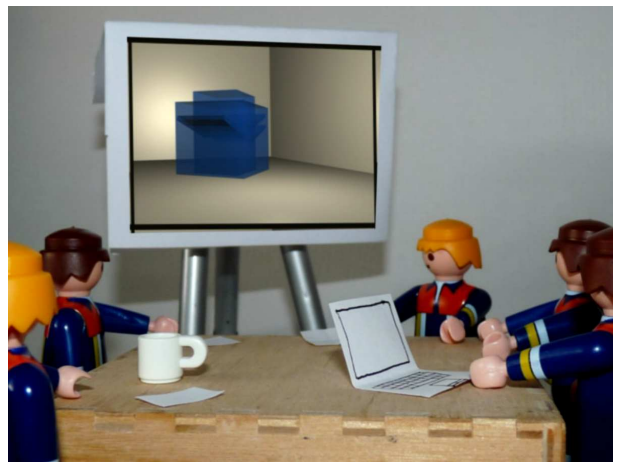

b. A virtual collaboration tool supporting a design meeting.

Figure 4.1 Animation stills from two of the four example storyboards.

participants the idea that there is room for improvement, exploration and modifications. In spite of the low fidelity however, the storyboards still effectively communicate the VR application, its purpose in the PDP and the practical context in which it is used.

\section{Proceedings}

The structure of the workshop is illustrated in figure 4.2. After introducing the purpose and structure of the workshop and providing some background information about $\mathrm{VR}$, the participants were shown the four example animated storyboards.

The participants were first given the key frames of the example storyboards (between 8 and 10 frames for each storyboard), along with additional technology frames depicting VR technologies and empty frames allowing for custom events to be added. Each participant was asked to create an individual storyboard describing a design activity or event based on one of the example storyboards, or one that they find particularly interesting or challenging in practice. Figure 4.4a shows one of the individual storyboards created during the workshop.

After creating individual storyboards, the participants formed four groups and presented their storyboards within their groups. The groups were formed based on the disciplines of the participants; each group included as many disciplines as possible. The participants were asked to merge relevant topics from the individual storyboards into group storyboards, and provide the group storyboards with more detailed descriptions of why, when and by whom VR is applied. Figure 4.4b shows one of the group storyboards created during the workshop. In the final stage of the workshop the groups present their ideas in a five minute presentation and discuss the results with the researcher and the other group members. To wrap up the workshop, a voting round is held in which participants are allowed to place positive or negative labels (4 each) on the storyboard posters.

Throughout the workshop the storyboards served their purpose of supporting the generation of application scenarios as well as the presentation and discussion of these scenarios. Despite their low fidelity, the storyboards facilitated communication between the participants about a topic with which they were not very familiar. After a short period of getting used to working with the cards, participants found their own way of structuring them into a desired scenario and presenting this as a coherent story. The resulting scenarios show a wide range of applications, 


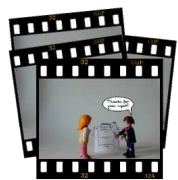

\author{
Example \\ animated \\ storyboards
}

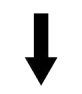

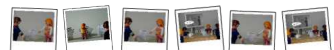

Let

Selection of

key frames

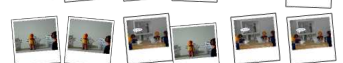

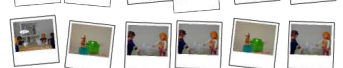

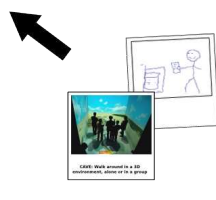

Addition of tech frames and custom frames

12 individual

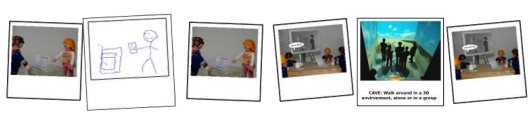
storyboards

\title{
$\downarrow$
}
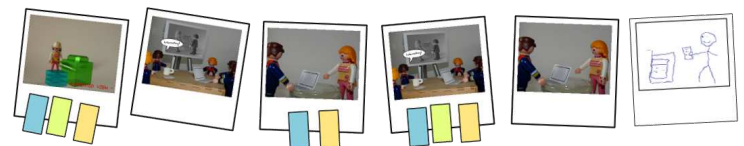

4 detailed group storyboards<smiles>[AlH2]</smiles>
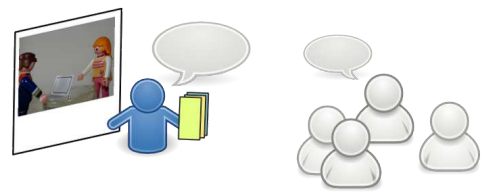

Presentation and discussion

Figure 4.2 A graphical outline of the exploration workshop. 
as well as a diverse range of presentation styles; some participants attempt to use as many pre-defined frames as possible, while others create an entire scenario from scratch, using a lot of sketches and notes.

\section{Results}

While the group storyboards are the final outcome of the workshop, the proceedings of the entire session have to be taken into account when defining the result of the workshop. The researcher therefore reviewed the individual storyboards, the group storyboards and video recordings of the discussions and presentations held throughout the session to define three themes that represent advantageous VR applications for the company.

1. Theme 1: Improve use context representation for usability evaluations - The current procedure for testing a new printer (or one of its components) isolates the printer from its use context (the test participant just sees the printer in an empty room). This is expected to influence the feedback given by the test participant. Designers expect the feedback from participants to be different and possibly more reliable when the test environment is more realistic.

2. Theme 2: Improve contextual inquiry data capturing and visualisation - 3D capturing techniques are used to capture a real-life use context and interactively present this use context to designers. An application similar to Google Street View is envisioned, allowing for a better transfer of field knowledge into the design team.

3. Theme 3: Precede physical prototyping with virtual prototyping - Virtual prototypes can be used to evaluate the design of error handling procedures (such as the replacement of machine modules), especially focusing on the physical aspects, such as margins, weight and pressure required to replace a module. Several procedures need to be tested this way before being implemented in a final design. The current method of using physical prototypes takes a lot of time and is expensive. Virtual prototypes could resolve these issues and may also be useful for training purposes.

Figure 4.3 illustrates how the twelve individual storyboards and four group scenarios contribute to these themes.

The researcher's analysis of the storyboards and the resulting definition of themes has been discussed with the participating designers in a follow-up session. Although all three themes describe a relevant challenge and a feasible starting point for advantageous VR applications, it was decided to focus on theme 1 as it directly addresses a use-related challenge, and because its core application is supported by two of the four storyboards. Themes 2 and 3 are considered less relevant for the particular focus of the research, which is the facilitation of user involvement in the early stages of the PDP. Theme 2, although addressing the use context, does not actually involve end-users in the design activity but focuses on gathering knowledge about end-users. Theme 3 primarily focuses on detailed engineering issues and requires more detailed design information than what is available in the early stages of the PDP.

\subsubsection{Application description}

Theme 1 has been used as the basis for the Virtual Printshop application. The purpose of the Virtual Printshop is to provide end-users with a realistic environment in which they can evaluate new printer concepts. Designers expect that a realistic environment will trigger different and 


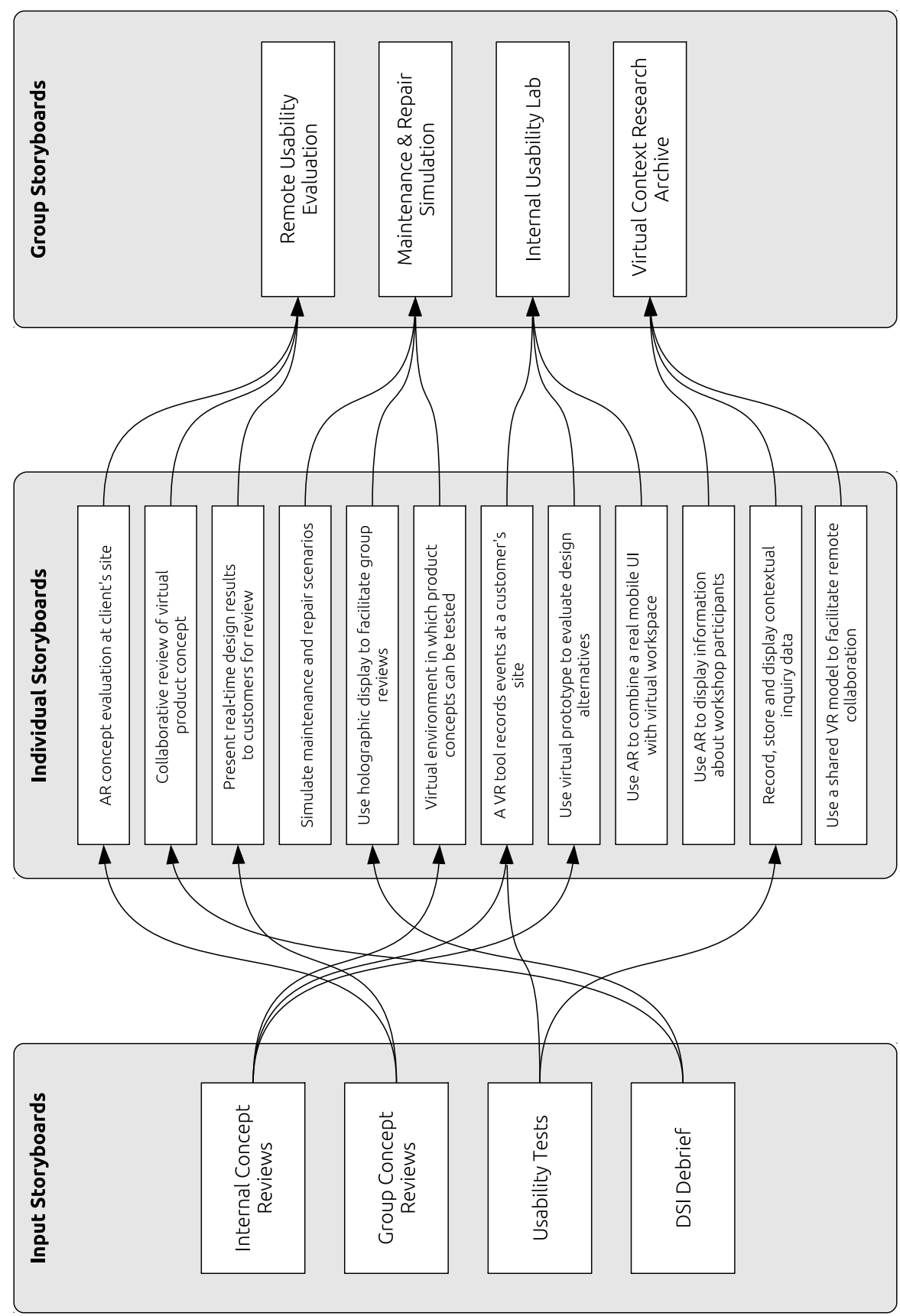

Figure 4.3 Results of the workshop. On the left it is shown how the four input storyboards influenced the individual storyboards. From the individual storyboards, group storyboards were created, focusing on the storyboard highlighted in bold. From the group storyboards the final direction for the VR application emerged. 


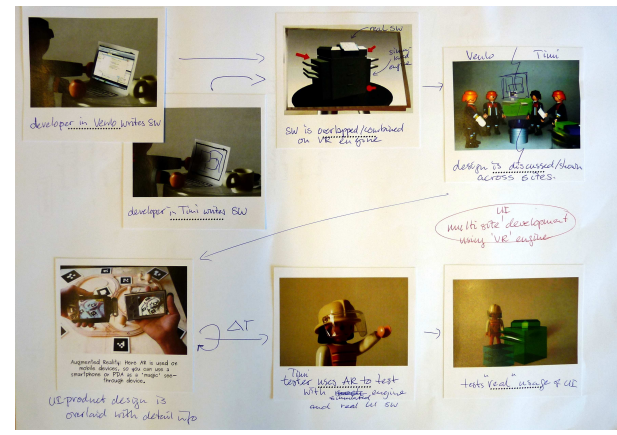

a. An individual storyboard created during the workshop.

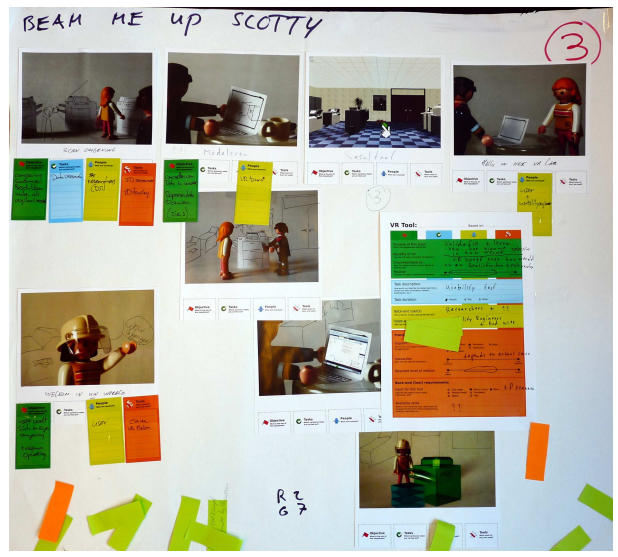

b. A group storyboard, showing the small cards that are used to describe the details of particular frames.

Figure 4.4 Storyboards created during the workshop.

possibly more reliable behaviour of end-users during a concept evaluation. Although a physical test lab is already available in the design department, it does not represent a realistic use context; it is an empty room with a clinical appearance, whereas a real use context typically consists of crowded printshops where phones are ringing and customers are calling for attention.

Furthermore, the current evaluations typically focus on specific product aspects, depending on what kind of prototype is available. For instance, in an early stage of the development, a fully functional printer is not yet available, making it difficult for designers to evaluate graphical user interfaces or user-product interactions. Designers currently solve this by providing the missing information verbally, e.g. 'a tray will open when you press this button' or 'imagine a light is blinking here, what would you do?'. VR technologies, in addition to providing a realistic use context, can facilitate these evaluations by providing virtual prototypes of future printers, presenting end-users with a more complete picture of the future use situation.

The envisioned Virtual Printshop consists of a virtual office environment in which end-users (or designers) can virtually move around. The environment should try to resemble real-life use environments, for instance by including objects or furniture encountered during client visits. The environment is interactive in the sense that the end-user can virtually operate printers (turning them on and off, or adding new paper). Furthermore, there are several context elements that add to the sense of realism, such as ambient sounds (phones ringing, people talking) and a queue of people waiting for their print job to finish.

\subsection{Development}

This part of the case study involves the development of the Virtual Printshop application that provides designers with a virtual use context that can be used for early product evaluations involving end-users. The functional prototype of this application is used in a test session to validate the application. 


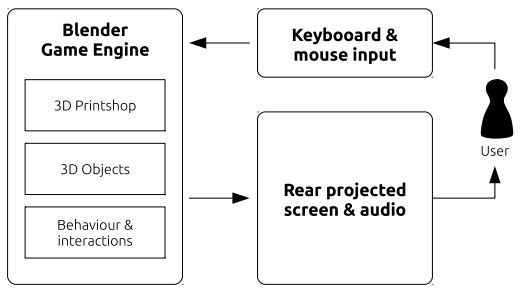

a. Diagram of the Virtual Printshop setup. The Blender Game Engine is used to model, control and render the virtual environment, which is projected on a large rear projected screen.

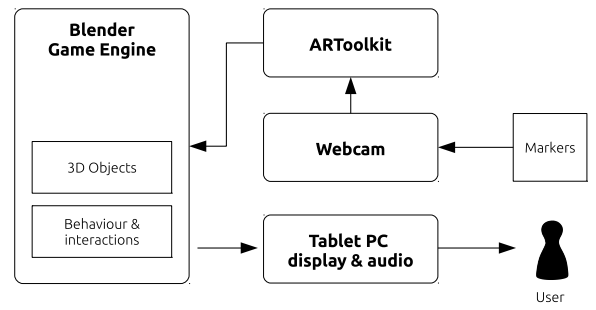

b. Diagram of the AR Printshop. The Blender Game Engine is connected to ARToolkit, which takes care of marker detection using a webcam on the tablet PC.

Figure 4.5 Diagrams outlining the software used in the two virtual printshops.

\subsubsection{Application prototype}

While the exploration workshop led to a clear description of what the Virtual Printshop should be able to do, there is no consensus about how this should be done. Two technical alternatives were discussed with the design practitioners. Augmented Reality (AR) could be used to place end-users in the virtual use context and let them act out work habits and task sequences. Alternatively, a fully virtual environment such as a CAVE or a relatively simple first-person game environment could provide a virtual environment in which the same tasks can be completed. In order for design practitioners to assess these technical alternatives, it was decided to develop and evaluate two application directions, namely the 'Virtual Printshop' and the 'Augmented Reality Printshop'.

1. The Virtual Printshop consists of a 3D virtual office that is projected on a large rearprojected screen $(3 \times 2 \mathrm{~m})$. Designers, positioned in front of this screen, use a keyboard and mouse to navigate a first-person perspective through the environment. The application runs on a standard desktop computer and uses the Blender Game Engine ${ }^{1}$ for rendering and controlling the interactive 3D environment (see figure 4.5a).

2. The AR Printshop consists of a tablet PC equipped with a camera. Pointing the tablet at a visual marker will display corresponding $3 \mathrm{D}$ models on the tablet display. This allows designers to physically move around while exploring the augmented reality environment, pointing at specific markers. The augmented reality is based on a combination of ARToolkit $^{2}$ and the Blender Game Engine (see figure 4.5b).

An existing printshop has been used as a reference for creating virtual models of office furniture, machinery, layouts and room decorations that provide a common basis for both applications. Some of the objects are interactive; the printer models have system states, such as 'printing', 'idle', or 'out of paper' that can be changed by user interactions.

In addition to evaluating the difference between the Virtual Printshop and the AR Printshop, we were interested in the required level of realism of the virtual contexts. Without proper references, it is difficult for design practitioners to indicate the level of realism they need for a

\footnotetext{
${ }^{1} \mathrm{~A} 3 \mathrm{D}$ authoring and development environment, see http://www.blender.org

${ }^{2} \mathrm{~A}$ generic augmented reality library, see http://www.hitl.washington.edu/artoolkit
} 

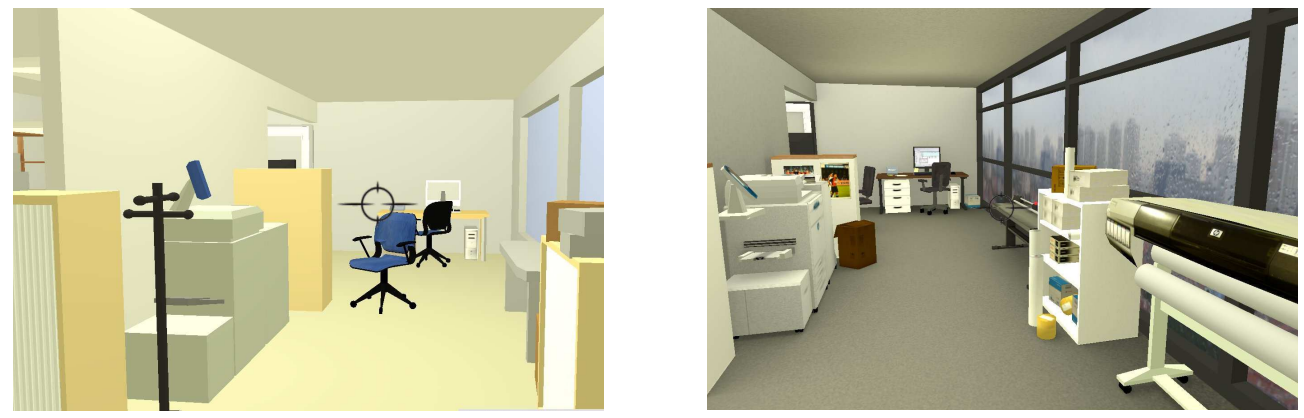

a. The 'Virtual Printshop' in low level of realism (left) and high level of realism (right).
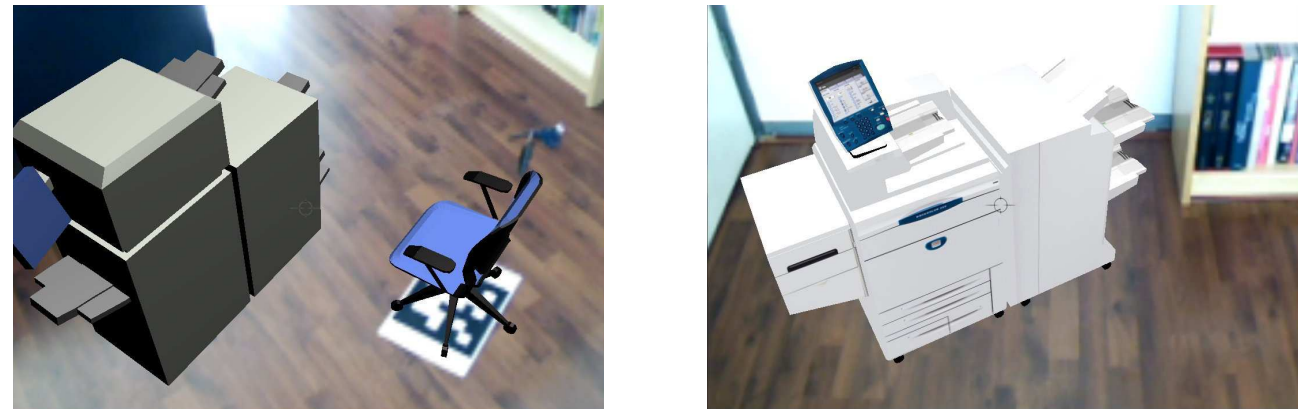

b. The 'Augmented Reality Printshop' in low level of realism (left) and high level of realism (right).

Figure 4.6 The two application prototypes and their levels of realism.

use context to be effective in product evaluation sessions. Without a sufficient level of realism users may not recognise an environment or objects, or may not take the evaluation task seriously. Creating highly realistic environments, on the other hand (visually, but also in terms of audio and interactions), is time consuming and therefore less feasible in the early stages of a design process.

To see how the level of realism affects the effectiveness of the VR application, both printshop applications were created with two degrees of realism. The high realism applications include visually rich objects (e.g. detailed geometry, photo-realistic textures and realtime shadows), 3D sound and interactive animated models (e.g. moving printer parts). The low realism applications use models with less detailed geometry, no textures, no shadows, regular stereo sound and lack animated objects. Figure 4.6 illustrates the different levels of realism used in the Virtual Printshop and the AR Printshop.

\subsubsection{Application validation}

The AR Printshop and the Virtual Printshop were deployed in a test case. The aim of this test case was to assess the effectiveness of product evaluations in a virtual use context and to compare the Virtual Printshop to the AR Printshop. 


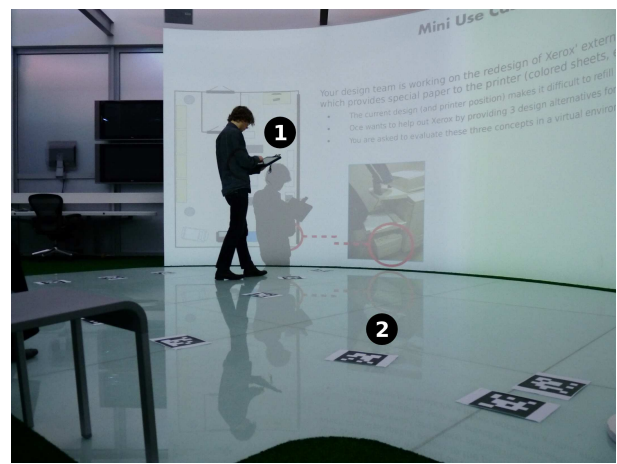

a. The Augmented Reality Printshop, in which a designer uses an augmented reality tablet (1) to walk around the augmented reality markers (2).

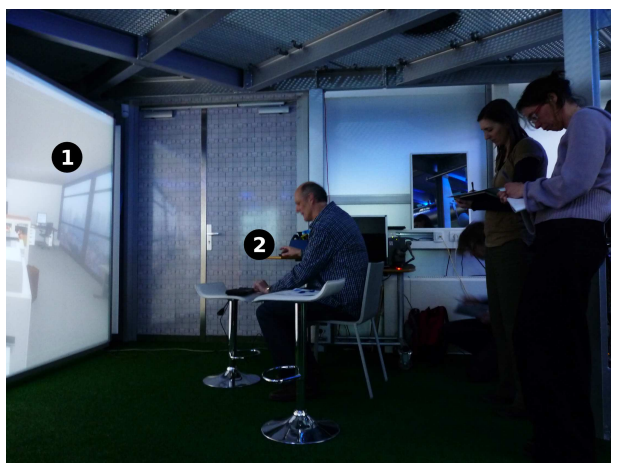

b. The Virtual Printshop, in which designers (2) operate a first-person perspective 3D environment projected on a large screen (1).

Figure 4.7 The two prototypes of the virtual printshop in use during the application review.

\section{Approach}

A group of four designers from the company was asked to carry out a product evaluation in both the AR Printshop and the Virtual Printshop, and to compare these sessions to the product evaluation sessions in the traditional test environment (i.e. a product evaluation in the dedicated usability lab). The fictitious topic of the product evaluation consisted of a new paper feed tray, for which three design concepts have been created. Each concept represents specific positions and opening mechanisms of the tray. This topic was chosen because it covers physical interactions between the operator and the product, as well as interactions between the user interface and the tray; operators have to be able to reach the tray, and the user interface should inform operators about an empty paper tray. While the product evaluation session should also include real end-users, it was decided to involve only designers because of the experimental nature of the applications. During the test session, designers who operated the virtual printshop (i.e. control the keyboard and mouse, or hold the AR tablet) temporarily acted as end-users.

The participants were subsequently introduced to 1) the high realism Virtual Printshop, 2) the low realism Virtual Printshop, 3) the high realism AR Printshop and 4) the low realism AR Printshop. The designers spent about thirty minutes in each of these four virtual printshops, carrying out a use scenario to evaluate the different paper tray concepts. The use scenario, which was the same throughout the evaluation session, consisted of the following steps:

1. A printer runs out of paper and switches to idle

2. The operator collects a new pack of paper

3. The operator opens the tray and inserts new paper

4. The printer resumes its print job

The participants were given forms (see appendix A.1) to write down feedback on the different paper tray concepts. Figure 4.7 shows the group of designers as they carry out the evaluations in the two different virtual printshops. Figure 4.8 illustrates the key elements of 


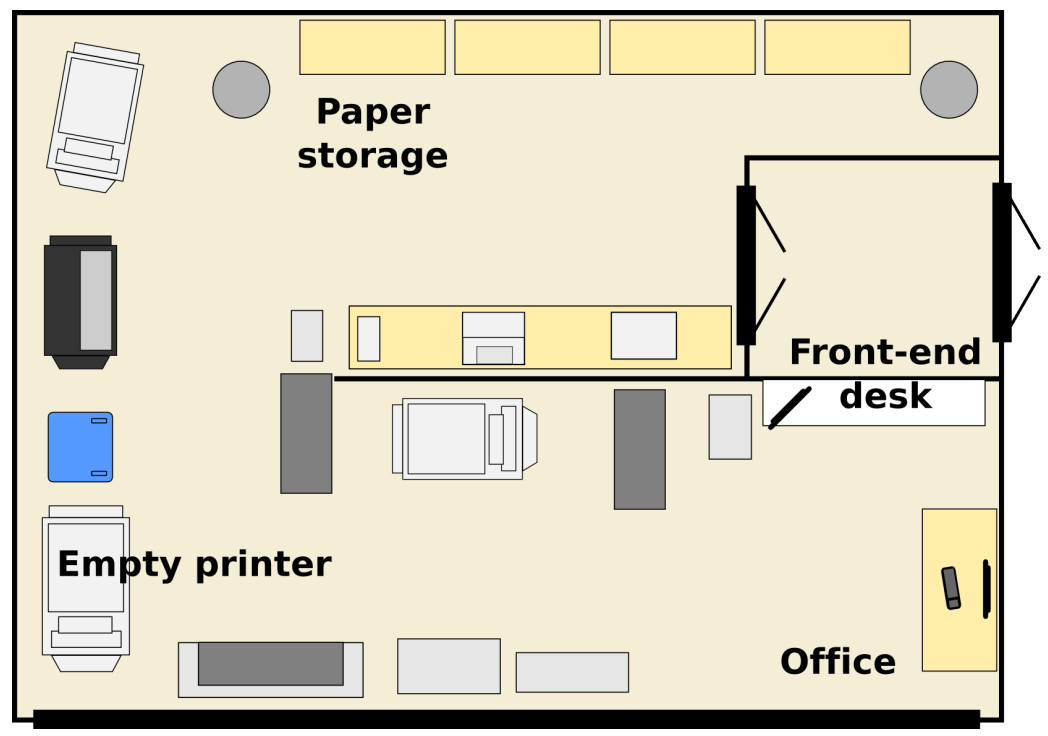

Figure 4.8 Layout of the printshop. During the product evaluation, the printer on the lower left runs out of paper and needs to be refilled. The participants collect a new pack of paper from the paper storage. A queue of customers forms at the front-end desk during the evaluation.

the use scenario in a layout of the printshop. After completing the evaluation sessions a group discussion was held to gather feedback on the different types of virtual environment.

\section{Results}

The validation session did not result in a lot of insights regarding the particular paper tray concepts. As the participants were mostly discussing the different VR applications rather than the content of the use case, only few things were written down on the evaluation forms. In spite of this lack of feedback, the session provided a lot of insights in the differences between the Virtual Printshop and the AR printshop, as well as the differences between the two levels of realism of both printshops. Transcripts (based on video recordings of the session) of the evaluation session as well as the subsequent discussion have been analysed using an open coding approach. The categories listed in tables 4.2 and 4.3 have been used to structure the statements and observations of the evaluation session and the discussion respectively. Appendix A.1.2 contains the resulting categorised set of statements and observations. The remainder of this subsection will discuss the outcomes and refer to these statements when applicable.

Both printshop applications allow users to move from one printer to another, and to include workflow elements such as receiving printing orders from customers, post-processing a print job or doing administrative tasks on a computer. One of the major advantages of the test environment being virtual is that the layout of the room and the types of printers can easily be changed. This allows designers to quickly evaluate how different room layouts or changes in machines or staff affect an end-user's workflow. In addition to doing product evaluations and workflow analyses, designers indicated that the applications can also be used for generating and quickly evaluating new ideas or for communication purposes (e.g. interactive demonstrations 


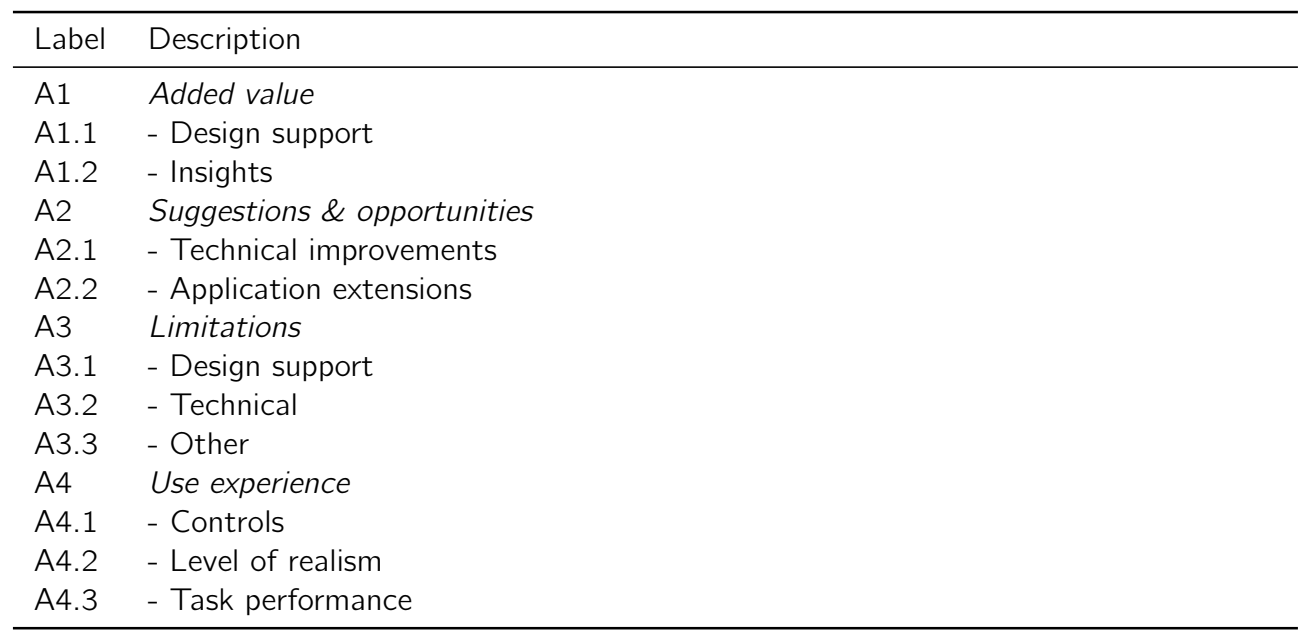

Table 4.2 Categories identified for the two evaluation session transcripts. This structure has been used for the 3D Printshop evaluation (referred to as 'A') as well as the AR Printshop evaluation (referred to as 'B'). Appendix A.1.2 provides a full list of statements and observations.

of new products) (statements $A 1.1 .4$ and $A 1.1 .5)$

With respect to the differences between the Virtual Printshop and the AR Printshop, it was found that designers preferred the Virtual Printshop over the AR Printshop (statements C2.1.1 and C2.1.3). Designers indicated that the augmented reality approach does not really achieve a feeling of being in the printshop; the restricted view through the tablet computer, the lack of walls and the sudden 'popping up' of objects in the augmented reality environment prevented the participants from staying 'immersed' in the virtual world (statements B3.1.2 and B3.1.3). A benefit of mixed reality on the other hand is that it also simulates physical interactions; designers had to kneel down in order to reach lower paper trays. However, such physical and ergonomical aspects are more easily tested through wooden or paper mockups, limiting the added value of $\mathrm{VR}$ in this area.

With respect to the difference in levels of realism, it was found that the level of realism of products should be high, comparable to the high-level demo. A printer in the low-realism printshop triggers less feedback than a highly realistic printer (statement A4.2.11), and it makes it difficult to assess the dimensions of the object (statement A4.3.2). The realism of the context is less important, but should be slightly higher than the low-level demo (e.g. add shadows, visual cues for interaction). Participants agreed that it is a matter of experience to know what to include (or not) in the context (e.g. is a clock a part of the workflow?). In context visualization, the layout adds sufficient reference for recognizing a certain printshop; chairs do not need to be a 1:1 copy of the real chairs, as long as there are chairs on the correct location in the room (statements A4.2.2, A4.2.10, C1.1.3 and C1.1.4). Apart from visual realism, participants also noted that sound significantly affects the sense of realism. The low-realism sound (on or off) was considered confusing, even though it provides a clear indication of printer status. It was concluded that sound should be either realistic (stereo, 3D, interactive) or completely left out (statements C1.2.2 and C1.2.5).

Following these findings, it is concluded that the application facilitates the anticipated 


\begin{tabular}{ll}
\hline Label & Description \\
\hline C1 & Level of realism \\
C1.1 & - Visual \\
C1.2 & - Audio \\
C1.3 & - Behaviour \\
C1.4 & - General \\
C2 & Level of virtuality \\
C3 & Tool chain requirements \\
C3.1 & - Integration \\
C3.2 & - Functionality \\
C3.3 & - Issues \\
C3.4 & - Usability \\
C3.5 & - Suggestions \\
\hline
\end{tabular}

Table 4.3 Categories identified for the discussion transcripts. Appendix A.1.2 provides a full list of statements and observations.

design task, namely early stage product evaluations. However, while the application initially focused on evaluating physical aspects (namely the accessibility of paper trays), designers indicated that the application should rather focus on the evaluation of workflows and and room layouts. Furthermore, based on the feedback from the designers it is decided to focus on the realisation of the Virtual Printshop rather than the AR Printshop. It was also found that even with a lower level of detail, participants still recognise a use context, as long as there are sufficient references to the real-life environment.

\subsection{Deployment}

Having decided upon the Virtual Printshop as the most suitable application, the next step in the case study is to provide designers with appropriate tools to realise this application themselves. As explained in chapter 3, the realisation of the application requires preparational tools and execution tools. The execution tools required to 'run' the Virtual Printshop application are relatively straightforward; the primary functionality, a first-person 3D walkthrough, is provided by off-the-shelf software such as 3D engines and even certain CAD applications.

In the current case study, the most challenging aspect with respect to the realisation of the application lies in creating the Virtual Printshop's content using preparational tools, as the designers involved in the case study are not used to creating 3D environments. Furthermore, although they are experienced in modelling 3D geometry, it may be difficult to add behaviour to these models. The tool selection and tool evaluation therefore focus on providing the designers with appropriate preparational tools for creating the required application.

\subsubsection{Tool selection}

Tool selection depends on several aspects, such as the required level of realism of the resulting virtual environment, the available skills (e.g. modelling or programming the environment) and possibly the integration with other tools used in the PDP (e.g. to use data from existing model repositories). Given their experience with the application prototype earlier in the case study, the 
designers were able to contribute to the tool selection by expressing concrete requirements and preferences. Designers were introduced to three steps which are required to realise the Virtual Printshop application and the range of tools available for each of these steps.

- Geometry Modelling - This step involves the creation or importing of model geometry (including shapes, colours, materials, etc.) needed for the virtual environment. In the virtual printshop this includes printer models, furniture and avatars. Tools available for this step range from regular $3 \mathrm{D}$ modelling suites and CAD software, to simply importing existing models from internal or external model repositories.

- Scene Integration - Scene Integration involves the creation of a virtual room or area and putting the 3D geometry in this environment. In the virtual printshop the room consists of the printshop room, and the arrangement of printers and furniture within the printshop. Tools available for this step range from $3 D$ modelling suites and CAD software to dedicated interior decoration and layout software.

- Behaviour Modelling - The third step involves defining the interactive behaviour of objects and the environment. In the virtual printshop this includes the system behaviour of printers (e.g. being able to print and output paper) and the ability of avatars to form a queue at the printshop's desk. Tools available for behaviour modelling range from pre-programmed behaviour to visual programming or 'regular' programming and scripting languages.

In a short presentation the researcher explained to the participating designers how different tools used for each of these tasks lead to different levels of realism and virtuality. Higher levels of realism for instance require more complex modelling tools such as game engines, while low realism environments can be created with easy to use off-the-shelf interior design software. Sharing this information with designers enables them to assess the trade-offs between application characteristics and tool requirements, but also allows for a comparison between the tools needed to realize the VR application (the VR tool chain) and the tools already available within the company. Taking this information into account, the participating designers were able to compose a tool chain and allocate tool chain components to specific departments or disciplines.

- Geometry Modelling is allocated to product designers who already work with CAD models. During the prototype session it was found that some objects, such as printers, should have a relatively high level of realism. These models could therefore be directly imported from the company's existing CAD database. Other objects, such as furniture, have lower requirements with respect to realism (or similarity with a real-life environment) and could therefore be imported from generic 3D databases, such as Google 3D Warehouse ${ }^{3}$.

- For Scene Integration, designers prefer a low threshold and easy to use tool rather than a more flexible but complex tool such as a generic game engine. Interior decoration software such as SweetHome3D ${ }^{4}$ provide a user friendly way to create virtual environments and allow users to import other 3D assets (e.g. printers and furniture). This part of the tool chain would be used by usability engineers, who are usually in charge of arranging product evaluations.

${ }^{3}$ An online model repository, see http://sketchup.google.com/3dwarehouse

${ }^{4} \mathrm{~A}$ free interior design application, see http://www.sweethome3d.com 


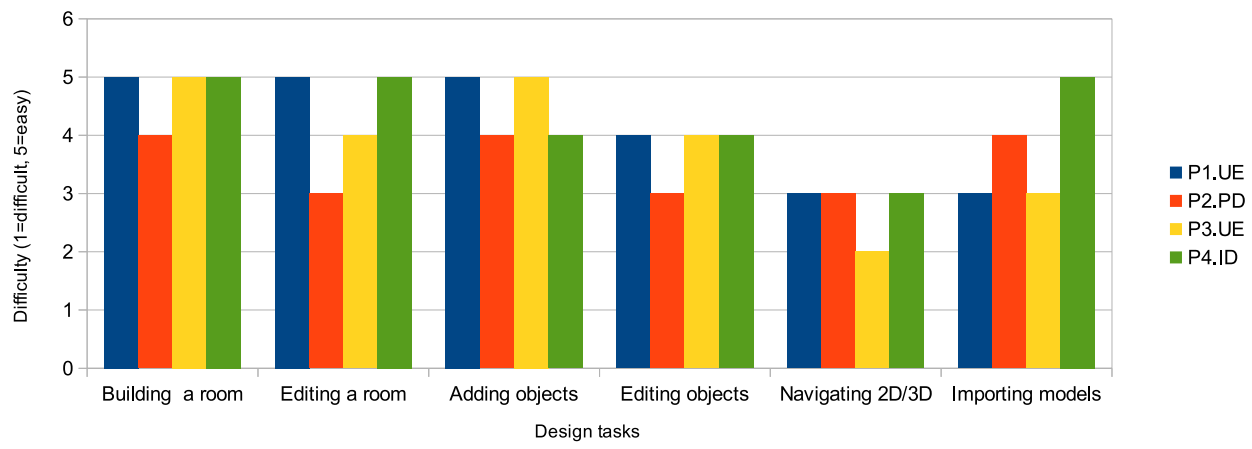

Figure 4.9 Assessment of various sub tasks in the first tool evaluation workshop.

- The designers indicated that Behaviour Modelling can be allocated to dedicated prototypers (designers trained in creating interactive software prototypes or mockups), who are already available in the design department. Given their experience with software prototyping the Behaviour Modelling tools can focus on functionality and flexibility rather than ease of use. It was decided to use the Blender development environment as a starting point for realising this part of the application.

The resulting tool chain consists of CAD tools and model databases for geometry modelling, SweetHome3D for scene integration and Blender for modelling the behaviour of interactive objects.

\subsubsection{Tool evaluation}

The tool chain was verified in two follow-up workshops. In the first workshop designers, usability engineers and prototypers were involved in carrying out their respective parts of the tool chain. Designers and usability engineers used SweetHome3D for creating virtual environments, which they had to do based on, for example, a floorplan and photos of a reference environment. Assets such as furniture and printers were imported from internal CAD databases as well as public databases such as Google 3D Warehouse. After the assignments the participants were asked to fill out an evaluation form (see appendix A.2.1). The results of these forms are included in appendix A.2.2. It is shown that even without specific training in 3D modelling, designers were able to import models from databases and put them in a virtual environment created from scratch without significant efforts (see figure 4.9).

The virtual environments resulting from this workshop were used in a subsequent workshop in which an experienced prototyper used the Blender Game Engine to add behaviour to these environments (e.g. ability to walk through the environment, interact with objects, etc.). Adding behaviour to this environment turned out to be difficult even for experienced prototypers. While it was expected that prototypers would be able to use a generic Game Engine for this, it was found that the learning curve of these tools (in this case the Blender Game Engine) is quite steep. In addition to the steep learning curve it should be considered that the tool will not be used on a daily basis. This bottleneck could be addressed either by providing designers with 
easy to use programming tools for creating interactive prototypes or by outsourcing this task to experts such as dedicated prototypers (within or outside the design department).

\subsection{Conclusion}

The case study involved the development of a Virtual Printshop, a VR application that improves the reliability of product evaluations in the early stages of the development process by providing a more realistic test environment. The application has been validated successfully in a practical test case, leading to the following findings.

- The main role of the virtual environment is to provide a use context in which the evaluations take place. Context cues, such as ambient sounds or random events (e.g. phones ringing), contribute to the sense of a realistic environment.

- The virtual environment also helps with visualising the workflows of printer operators. This in turn provides designers with insights into how workflows are affected by the room layout or by the performance of individual printers or other machines.

- In product concept evaluations with end-users the virtual environment should be recognised by end-users but it does not necessarily need a high level of realism.

- The first-person 3D walkthrough environment is considered sufficiently effective for this application. The setup can be extended with e.g. motion tracking techniques to physically connect end-users to virtual avatars, or a third-person perspective to improve the insight in ergonomic aspects.

The application can be realised using off the shelf software and hardware. The primary bottleneck lies in the definition of interactive behaviour of objects in the environments. Although experienced (prototype) programmers were available in the company involved in the case study, this task is considered the most challenging aspect of realisation. Other elements of the application, such as 3D models can be modelled using present tools and skills, or acquired from external model repositories.

This chapter described the first implementation of the case study specification method introduced in chapter 3. Overall the method successfully structured the case study. However, in the exploration and the development phases, additional efforts were required to refine the VR application, and to decide on the technical implementation of the application (e.g. should it use augmented reality or a fully virtual environment). It was initially expected that such fundamental decisions would be made during the workshop, rather than during prototype development. While this was not found to be an issue for the remainder of the case study, it should be taken into account that the results of the exploration workshop may not always provide sufficiently detailed results to make such decisions. 



\section{$5 \quad$ Case Study 2}

The second case study describes the development and deployment of Virtual Personas. Traditional personas are written 'user profiles' that help designers with generating or reviewing new product concepts from the perspective of a particular type of user. Virtual personas are 3D avatars that live in a virtual world in which they can act out scenarios. As such, virtual personas are combined with scenarios, together providing an integrated view of future use situations. This chapter describes the identification of this VR application, the development of a prototype and the realisation of the application within the context of the company involved in the case study.

\subsection{Introduction}

The core activities of the company involved in case study 2 focus on the development, production, marketing, and sale of medium and heavy-duty commercial vehicles. By combining standardised vehicle platforms with customer specific components such as specific cabins, axle configurations, or drive trains, the company is able to meet each customer's individual specifications and transport requirements.

Throughout the case study the researcher is primarily involved with designers from the vehicle definition department. This department is in charge of defining the core vehicle properties such as ergonomics, safety or driver comfort. The product planning department provides the vehicle definition department with insights in current market needs, not only from vehicle drivers, but also from vehicle buyers. The insights are gathered through interviews and field studies carried out by the product planning department. After defining the initial specifications of each property, expert teams (consisting of experts from various departments) further elaborate these designs, after which the development gradually transfers to the engineering department.

Both the vehicle definition department and the engineering department make use of prototypes and field tests to validate product concepts prior to the engineering and manufacturing stages. The existing methods, however, depend on physical prototypes of e.g. an instrument panel or a new cabin design, making it difficult to apply these methods in earlier stages of the development process. One of the areas in which the company expects VR to be effective is in enabling the company to conduct concept validation in an earlier stage of the PDP.

\section{Chapter outline}

The company's expectations with respect to VR and UCD are further investigated and explored during a kick-off meeting and the exploration workshop, as described in section 5.2. Section 5.3 introduces the virtual persona application that resulted from the exploration workshop and outlines the development and validation of the application in a practical use case. In section 
5.4 the deployment of the application within the company's development process is discussed by selecting and evaluating supporting tools.

\subsection{Exploration}

The exploration phase covers the exploration of potentially interesting applications of VR for the company, eventually leading to the definition of the Virtual Personas. The following subsections outline the ideation and selection process that preceded this definition.

\subsubsection{Kick-off}

As in the first case study, the kick-off meeting is a preliminary meeting that serves two goals. Firstly, it introduces the case study approach to the participating designers. In this presentation, results of the first case study were used to illustrate the process and its outcomes. Secondly, the meeting serves as a preparation for the exploration workshop by determining the focus of the case study.

To initiate a discussion about the focus of the case study, the researcher proposed several possibilities on how to improve the company's PDP. These possibilities were identified during the site visits and interviews held in the field study (see section 2.3).

1. Facilitate communication between the product planning department and the vehicle definition department to get a better insight in customer requirements.

2. Support concept prototyping activities in the early stages of the development process to speed up and increase the frequency of design iterations.

3. Improve the communication between the product planning department and customers, for instance by letting customers evaluate or discuss early stage product concepts.

In the subsequent discussion, the company expressed a strong preference for speeding up (and increasing the frequency of) design iterations that take place between the vehicle definition department, vehicle control department, and the testing department. Furthermore, the design iterations should take place in an earlier stage of the development process in order to prevent expensive or time consuming redesigns. The participants explicitly mentioned a driving simulator as a preferred solution for this challenge. A driving simulator could serve as a platform for the evaluation and validation of vehicle properties related to primary and secondary driving tasks, such as driver comfort, safety systems and in-vehicle information systems. In spite of this preference, however, it is concluded that the exploration workshop should have a broad perspective on what type of VR technologies to use, and how to apply them in the company's PDP.

\subsubsection{VR exploration workshop}

The VR exploration workshop is a collaborative session in which company participants identify and refine potential VR applications, as introduced in chapter 3 . While the method prescribes the use of animated storyboards to initially inspire the workshop participants with example applications, the current workshop features an alternative approach. As the company has a relatively specific expectation regarding the application (early stage concept evaluation and validation) as well as the solution (a driving simulator), it is considered useful to first broaden the 


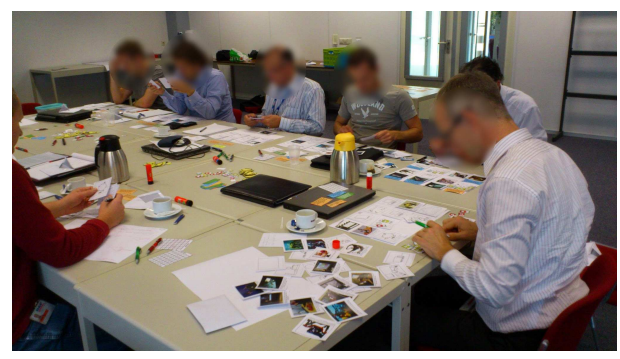

a. Workshop participants working on the storyboards.

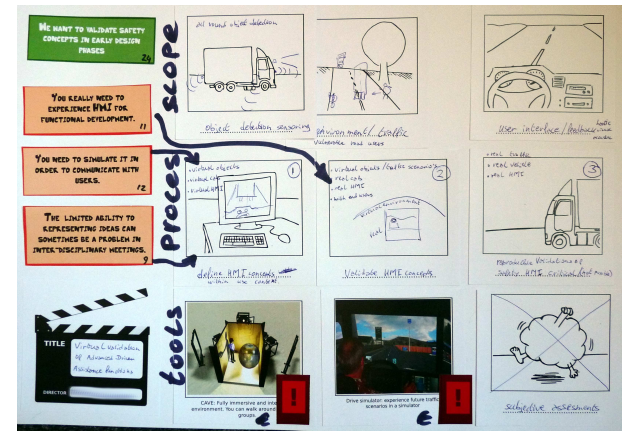

b. An individual storyboard showing the 'trigger cards' in the upper left corner.

Figure 5.1 The VR exploration workshop.

company's scope regarding VR technologies and their applications in the PDP. This alternative approach is introduced in the next subsection.

\section{Preparation}

As an alternative to the example storyboards the researcher created 40 trigger cards. The trigger cards depict quotes and observations gathered during the preliminary interviews and the site visit. The quotes and observations have been categorised into problems, statements and opportunities related to e.g. user involvement, communication issues between departments, or opportunities with respect to improving present design activities. The researcher primarily collected 'explicit' quotes and observations in order to elicit a response from the workshop participants.

During the workshop participants are asked to use two to three of these trigger cards as a starting point for their individual storyboards. The storyboards should illustrate how VR could address the trigger cards, for instance by solving a problem, by supporting or bunking a statement, or by addressing an opportunity.

\section{Proceedings}

The three hour workshop took place at the company's premises and involved eight participants from various departments, including three members of the vehicle definition department, one of vehicle control, one cabin designer, an HMI expert, an ergonomics expert and a system integrator. After a short introduction of the research project, the researcher outlined the purpose of the workshop and explained what role the trigger cards and storyboards would play in it. Each participant was shown each card (40 cards in total) by passing them round in the group. After reading all the cards, the participants were asked to select two or three 'interesting' trigger cards and use them as a starting point for the individual storyboards.

After completing the individual storyboards (which took about 45 minutes), the facilitator reviewed the storyboards and created three groups based on similarities in storyboard themes and content. After a short break the participants started to work on the group storyboards. Within each group, the individual storyboards were first presented to each other to identify similarities. These discussions helped shape the 'group' identity, and understand each other's views on the 
storyboards. After discussing the individual storyboards, the group members started to merge their ideas in a final group storyboard.

The session was wrapped up by letting each group present and explain their storyboard. While the group storyboards showed a lot of similarities at first sight, more detailed discussions (triggered during the presentations) uncovered ideas and stories that were not always explicitly mentioned in the group storyboards, but originated from the individual storyboards or were proposed ad-hoc. For example, although each group proposed to use some form of high-end $\mathrm{VR}$, they also pointed out several times that 'real-life testing' would always remain necessary;

"It was pointed out that they already did some experiments with virtual line of sights [...], but they were no longer used. It even took 'real-life' experiments to confirm the virtual tests." (quote from one of the group storyboard presentations)

As such the short presentations added a lot of insights to the group work and indicate the importance of thoroughly analysing the individual storyboards in order to determine whether or not high-end VR is actually useful or required here.

\section{Results}

Figure 5.2 visualises the relation between the trigger cards and the storyboards. The trigger cards helped participants create individual storyboards that address specific challenges and opportunities in the company's PDP, using various low-end and high-end VR technologies. The final group storyboards, however, show less diversity in VR applications; the individual storyboard applications were merged into three group storyboards that describe roughly the same storyline and mostly used high-end VR technologies such as CAVEs or immersive driving simulators.

While this confirms the company's preference for using a driving simulator in early stage concept evaluations, following only this direction may cause other potentially relevant applications (as shown in individual storyboards) to be lost. Therefore, a follow-up meeting was scheduled in which the researcher presented his interpretation of the individual storyboards and the group storyboards in three themes, as explained in the next subsection.

\subsubsection{Application refinement}

A more thorough analysis of the individual storyboards led to the identification of three themes for which VR applications were identified (improving user involvement, improving communication between departments and early stage evaluation and validation.) These themes provide the basis for specific VR applications. During the follow-up meeting, the researcher presented three concrete VR applications that respectively address these themes.

- Virtual Personas - Personas describe detailed, personal and specific archetypical users. These user models should become a 'real' person in the mind of the designers, and help them make appropriate design decisions 'on behalf' of that user. The company already created several personas, but finds it difficult to apply them in the development process. These existing personas can provide a starting point for the Virtual Persona application.

- Concept Review Room - With this application, designers from various departments can import their functional models (2D mockups, state diagrams, etc.) in an integrated 3D representation of the current project (e.g. a cabin or full truck). This model is a simplified version of the CAD model, acting as a template to merge the current state of concepts. 


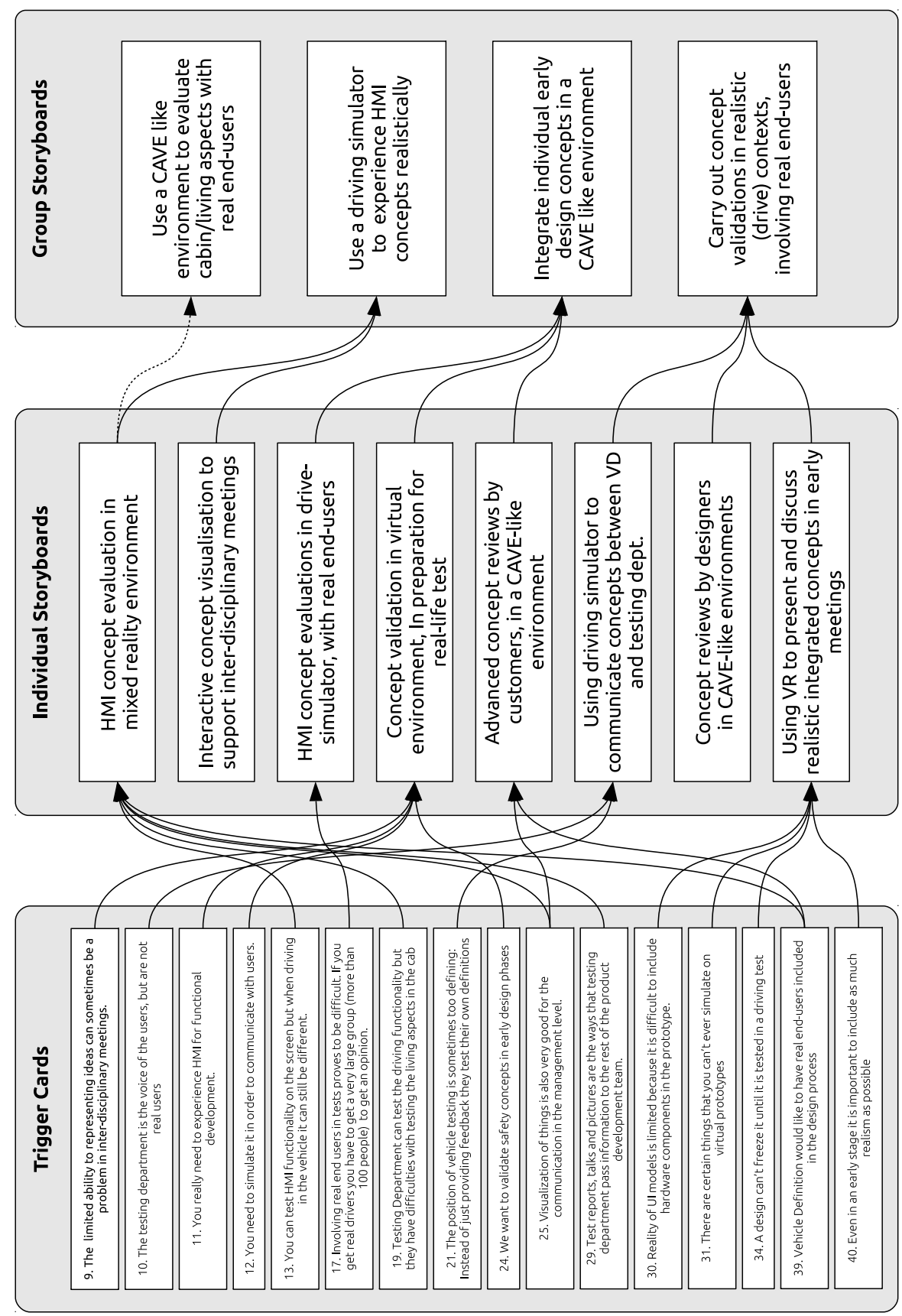

Figure 5.2 Visualisation of individual and group storyboards. 
- Driving Simulator - This application represents all the storyboards that described the company's original expectation with respect to VR, namely the use of a driving simulator for early stage evaluation and validation of concepts related to primary and secondary driving tasks. The presentation of this application included an overview of different forms of driving simulators, ranging from high-end immersive driving simulators to desktop simulator applications.

After introducing and discussing the three application directions, the meeting continued with selecting one direction for further use in the case study. There was agreement about the simulator being a less challenging or research-oriented direction, given the fact that both the technology as well as the related knowledge of the driving simulator application is already quite mature and familiar to the company. It was therefore agreed to focus on the first two application directions.

Both directions were found to be quite interesting; they provide a good combination of new possibilities and the potential re-use of existing infrastructure available within the company. The Virtual Personas application is interesting because it enables designers to involve end-users even though the company's policy prevents direct end-user involvement due to confidentiality restrictions. Furthermore, the application can potentially use the existing personas and matches the internal agenda that is already oriented towards improving user involvement. The Concept Review Room is technically challenging, but can use the company's existing facilities such as a $3 \mathrm{D}$ projection room. The application addresses relevant communication challenges between the vehicle definition, vehicle control and testing department.

During a meeting with company participants it was decided to focus on the Virtual Personas as the primary direction for the case study. Compared to the Concept Review Room application the Virtual Persona application was considered to be most in line with the topic of the research project as well as the company's internal agenda.

\subsubsection{Application definition}

The Virtual Persona application aims to provide a more vivid representation of traditional personas. A definition of personas is given in Calde et al. (2002): User models, or personas, are fictional, detailed archetypical characters that represent distinct groupings of behaviours, goals and motivations observed and identified during the research phase. These user models should become a 'real' person in the mind of the designers, and help them to make appropriate design decisions 'on behalf' of that user. An important aspect is the personality of a persona, which affects the goals of a specific persona.

Literature on personas mainly covers the creation of personas rather than the actual use of personas in a design process. As described in Blomkvist (2002), "the designers' knowledge of the persona's goals and the context (scenario), combined with design-common-sense, will move the design in the right direction". The lack of guidelines for using and applying personas also affects the current case study company. Although five extensive personas have been defined by the product planning department, they fail to reach the design and engineering departments of the company.

Seeing personas in a specific context, for instance in the form of a scenario, makes it easier to understand their actions and consequently help move designers in 'the right direction'. In Grudin and Pruitt (2002) it is argued that scenarios should be formed around personas to "[...] obtain a far more powerful level of identification and engagement [...]", similar to how people 
identify with characters from a soap opera series on TV. The resulting scenarios can be used for communication between design team members or to communicate with external stakeholders.

Similarly, in Carmichael et al. (2005), theatre techniques were used to effectively communicate personas and scenarios within a group of software designers. While human role playing transfers personas from paper to more 'engaging' stories, there are some drawbacks. Firstly, it takes quite some time to get used to acting out scenarios in front of colleagues. Alternatively, professional actors could be used, but this may lead to an increase in time and costs. Secondly, role playing still takes quite some imagination to 'see' the correct context in which a story is taking place. Thirdly, analysing the data from such sessions is time consuming; raw video has to be analysed in order to extract information relevant for the project.

The Virtual Persona application provides a virtual environment in which (present or future) use scenarios can be acted out with virtual personas. The application combines the advantages of role playing (resulting in engaging stories) with advantages of VR, namely the explicit and interactive visualisation of future use situations. Depending on the starting point, the application can be used for concept generation as well as concept evaluations. Regardless of the purpose of the application, designers will be stimulated to reason from the perspective of specific personas.

\subsection{Development}

This section describes the development of a functional prototype of the Virtual Persona application. Two main development iterations of the application are described, explaining how each iteration provides insights into several application parameters. The final functional prototype is used in a test session to validate the application.

\subsubsection{Application prototype}

The Virtual Persona application allows the company's designers to create and review use scenarios featuring virtual personas. To facilitate collaboration between designers during the creation and review of these scenarios, the technical setup for the application resembles a theatre layout; a large screen is used to present a group of designers with a live view of the virtual environment in which the scenarios are acted out. This virtual world provides the setting in which the use scenarios take place. The application prototype provides an urban environment as well as a highway, a truck parking lot and a petrol station. Furthermore, the environment includes interactive objects, such as autonomous traffic and driveable trucks. Within the virtual world, virtual personas are represented by avatars. The different types of personas are represented by their clothes, physical appearance and facial features. Designers can control the virtual world and the virtual personas through a user interface (see figure 5.3). The user interface allows designers to control the avatars (e.g. their behaviour and movements in the virtual world), but also provides access to virtual world settings, such as lighting and camera viewpoints.

The above elements have been implemented by the researcher using the Blender Game Engine. This development environment supports the creation of the 3D models and virtual world as well as the definition of interactive behaviour of the avatars and trucks. Furthermore, its open architecture allows for experimental communication with external components such as the Kinect platform. 


\section{First iteration}

The first prototype of the application provided a virtual world with two different virtual personas, represented by avatars, as shown in figure 5.3. The primary application parameter that was investigated in the initial prototype was the interaction method for controlling the avatar. A motion tracking solution, based on the Microsoft Kinect hardware, enabled the avatar to mimic the gestures and movements made by a designer. This was expected to contribute to a natural experience in creating use scenarios. For example, a designer could sit on a chair and make 'driving movements' to let the avatar act out a driving scenario.

During a first review session, the application prototype was introduced and four company participants interacted with the Virtual Persona application. The review session featured a simple use case concerning the evaluation of new cabin lighting control concepts; the aim of the session was to determine how different personas would use the new control concepts. Two main issues were identified in a post-session discussion between the researcher and the participants. The first issue is that the personas were not sufficiently introduced before using the tool. The virtual avatars are simply dropped into the virtual world, without any introduction. Secondly, the current method of interaction with the avatar (motion tracking) may not be the most useful one. Apart from the motion tracking implementation not being sufficiently accurate, the designers did not see any added value in being able to control the avatars through gestures and motion tracking. The use of mouse and keyboard controls was considered more effective; this would allow the designers to position the avatar anywhere in the virtual world by simply clicking on the desired position. Furthermore, it would enable more detailed control of the avatar's local movements (e.g. directing an avatar to press a button by clicking on that specific button). A more extensive (on-screen ${ }^{1}$ ) user interface could provide access to these functions.

\section{Second iteration}

The results of the review session were used to further refine the application prototype. The following application elements were changed:

- Avatar controls - The motion tracking interface for controlling the avatars is replaced by mouse controls offering two modes of operation. In 'automatic mode', designers use a point-and-click method to tell the avatar to walk from $A$ to $B$, or to sit down on a truck seat. Built-in animations allow the avatars to carry out these commands. In 'manualmode', designers use avatar handles to manually position the limbs of the avatar. The avatar's skeleton structure automatically deforms accordingly, using inverse kinematics.

- User Interface - As requested by the designers after the first iteration, the user interface has been extended to an on-screen panel providing access to the main functions of the virtual environment, such as lighting, camera viewpoints and avatar actions. Furthermore, a screenshot function was added to allow designers to take screenshots of particular scenario events.

- Avatar details - The designers indicated that the virtual personas should be properly introduced before using them in a scenario. This has been achieved by creating a short introduction video of each persona (see figures 5.4a and 5.4b). Furthermore, the visual appearance of the avatars is improved by using more distinct clothing and facial textures, and by using different body models, as illustrated in figures $5.5 \mathrm{a}$ and $5.5 \mathrm{~b}$.

\footnotetext{
${ }^{1}$ The initial prototype did not have any on-screen graphical user interface elements, making it difficult for untrained users to operate the application.
} 


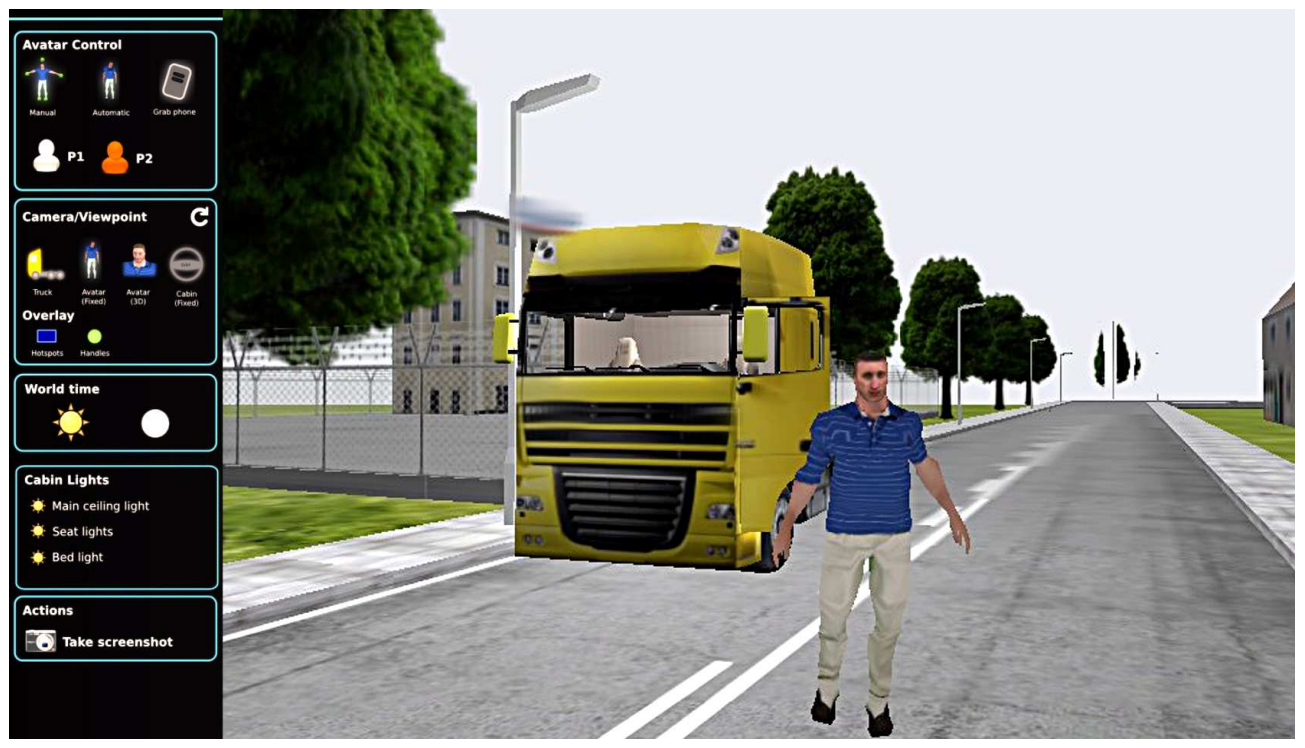

Figure 5.3 A screenshot of the application prototype. The avatar represents a persona and can be used to act out use scenarios in the virtual world. The on-screen user interface (on the left) was introduced after reviewing the first prototype, and provides access to common world settings, camera viewpoints and avatar actions.

In addition to these technical changes to the application prototype, the review session also indicated that the use case for the application should be further refined. In order to evoke different interactions from different personas, the interaction should be challenging or at least provide personas with a choice; the current use case however only allows personas to turn the lights on or off. For the application validation step, a more extensive and challenging use case was defined.

\subsubsection{Application validation}

The purpose of the virtual persona application is to let designers experience product concepts from the end-users' point of view, allowing them to review and improve the concepts. In the application validation step, the Virtual Persona application is deployed in a realistic design session in order to answer three questions, namely 1 ) does the session stimulate participants to put themselves in the shoes of end-users, 2) what kind of insights are gained from the session, and 3) what is the added value of using VR in this session?

The application validation comprises two identical one hour sessions, each involving four different participants from the company. Session participants include designers and engineers who will work on a fictional design case defined for this session. The design case concerns the idea of developing a new device to control various truck functions, such as the lights and the central locking system. The feasibility of this idea is to be assessed by the participants in this session, who are experts in specific areas such as mechanical engineering or $\mathrm{HMI}$ design. In particular, the designers and engineers are to evaluate the concept from the perspective of two 


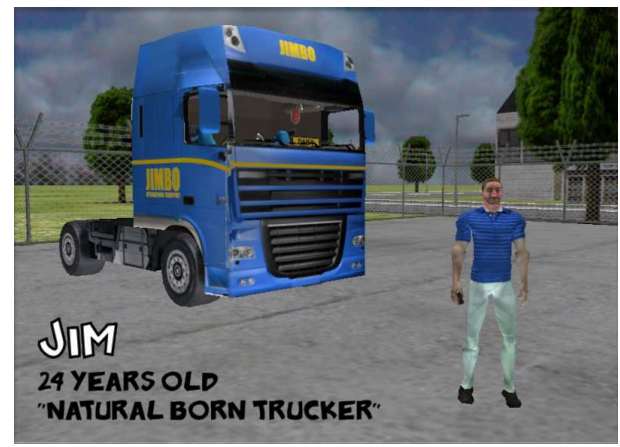

a. The 'young driver' persona.

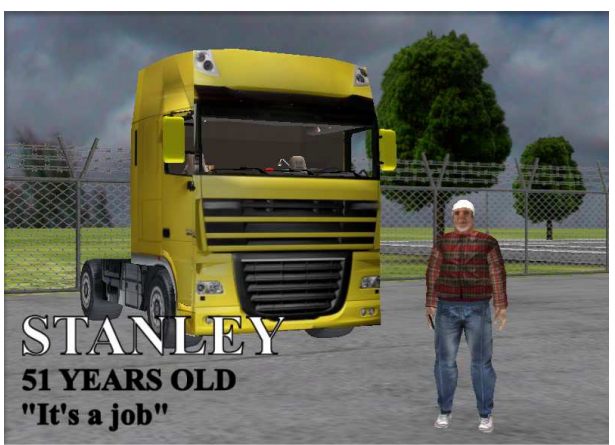

b. The 'experienced driver' persona.

Figure 5.4 The second iteration of the prototype featured more detailed personas that were introduced by short videos. The avatars representing the personas feature different textures and different body models.

personas, embodied in virtual avatars.

\section{Approach}

The two sessions are facilitated by the researcher, who will make sure that the following steps are carried out.

1. Case Description - The researcher welcomes the participants and explains that the goal of the session is to explore the opportunities of the new product concept. The concept is still in a very early phase, so the participants are asked to assess its feasibility (in terms of usefulness, functionality) but also to explore new functionality.

2. Scenario Setting - A very short scenario is introduced. The scenario focuses on a particular part of the day where the new concept could be used; near the end of the day, when a driver has to spend the night in his truck.

You are near the end of a long driving day. Because of driving time regulations you are forced to spend the night at a trucker's parking space near the highway. It's around 17:00 when you arrive at the parking space. There is a petrol station and small snack/restaurant nearby. After parking your truck, the evening starts...

3. Brainstorm - After the scenario stops, the participants are asked to brainstorm about what tasks a driver would do in this period of time, and what events could take place. Participants write down the tasks and events on cards and lay them out on a table.

4. Persona Introduction - The next step is to introduce two personas that will be used to create use scenarios. A short 1-minute video of each persona (Jim and Stanley) is shown. Questions about the personas can be answered by the facilitator. The facilitator then asks the participants to create two scenarios, using the perspective from the two personas. The virtual persona tool is to be used to create and visualise this scenario.

5. Scenario Generation - The task and event cards can be used to prepare a scenario sequence that can then be acted out in the virtual environment. The virtual environment 


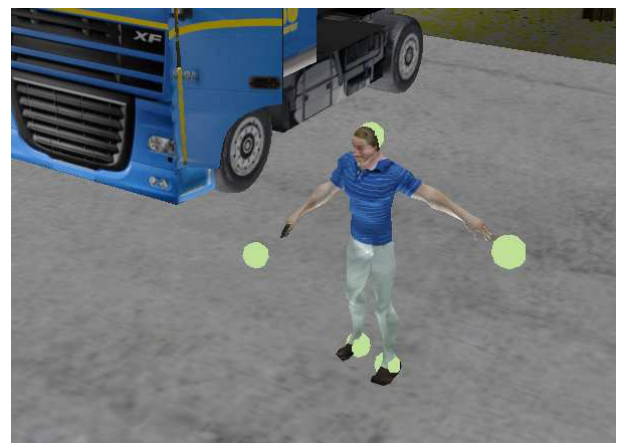

a. A virtual persona showing 'handles' to manually control limbs.

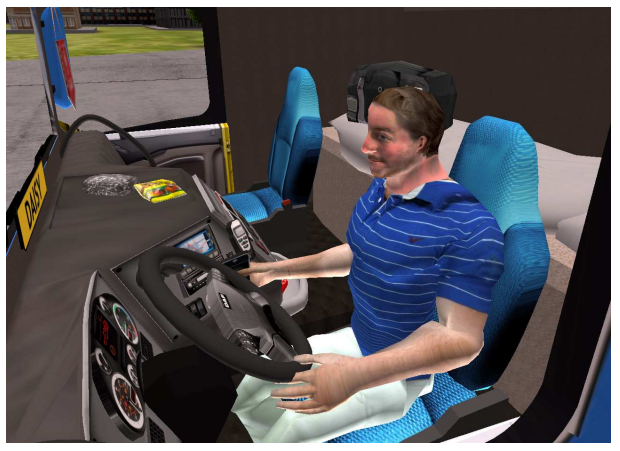

b. A virtual persona positioned on the driver's seat.

Figure 5.5 In the second iteration of the prototype the motion tracking input is replaced by more detailed mouse interaction that allows designers to control individual limbs using 'handles' and to automatically position personas.

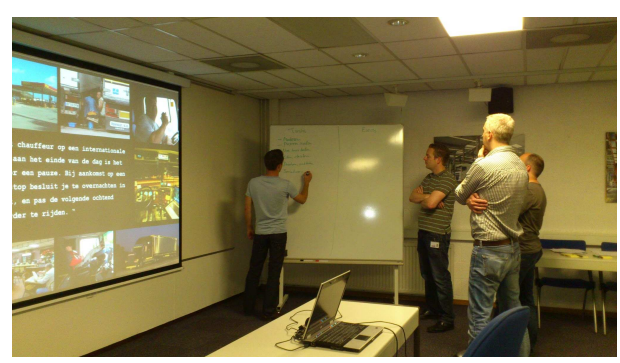

a. Designers compiling a list of tasks and events.

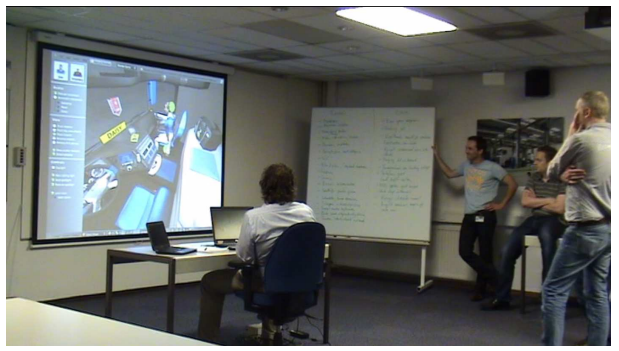

b. Designers acting out scenarios using virtual personas.

Figure 5.6 The application validation session.

is operated by a voluntary participant, supported where needed by the facilitator. A time frame of 20 minutes is available for each of the personas, so the participants will spend about 40 minutes on creating the scenarios.

\section{Proceedings}

The sessions started with a short introduction and explanation of the session, a short brainstorm about the scenario, followed by an introduction of the two personas and finally using the virtual environment to create scenarios. After each sub session, the participants were asked to fill out an evaluation form (see appendix B.1.1).

The first part of each session mainly consisted of a brainstorm about what could happen in the prescribed scenario time frame (arriving at a truck stop around 17:00, leaving the next morning around 8:00). Participants wrote down tasks and events on a whiteboard next to the main screen, so they could use this list as input during scenario generation.

After the brainstorm, which took about ten minutes, the groups started creating the scena- 
rios. In each group, one participant was asked to operate the Virtual Persona application; they controlled the avatar movements, camera viewpoints and other virtual world settings. Both groups needed some time to 'get into the scenario', but eventually they succeeded to do so. It was quickly discovered that it is good to use a chronological order of events, so the groups usually started with the personas having dinner, followed by some recreational time and then preparing to go to sleep. Throughout the session, the virtual environment provided a background and reference point for the group discussion, without distracting participants from the matter at hand. Whenever the operator ran into limitations with respect to acting out very specific actions (e.g. playing a video game, brushing teeth or performing truck maintenance) the group would take over and verbally explain what would happen in real life.

While creating scenarios, none of the groups focused on the product concept all the time; it was sometimes highlighted, but overall the events and tasks were getting more attention than the product concept. Only the first one or two tasks were specifically chosen and explained with the product concept in mind. For example, both groups started with the persona getting out for dinner and using the product to lock the doors, and turn on/off the lights from outside. To prevent participants from drifting away too far from the initial topic, the facilitator sometimes stimulated participants to return to the topic by asking them to act out one of the tasks or events resulting from the brainstorm.

\section{Results}

The proceedings of the application validation session were captured on video and audio and the participants filled out an evaluation form after the session. In the evaluation form, participants were asked to reflect on the results of the session, and to compare the use of the Virtual Persona application to the traditional use of personas. The results of this evaluation are included in appendix B.1.2. Together with the audio and video recordings of the session, which have been used to review the scenarios that were created during the session, the evaluation forms help answer the three questions introduced earlier, namely 1 ) does the session stimulate participants to put themselves in the shoes of end-users, 2) what kind of insights are gained from the session and 3) what is the added value of using VR in this session?

With respect to the first question, the majority of the participants (6 out of 8 ) indeed felt that they had been evaluating the product concept from two different user perspectives (question 3.1), and all participants consider this activity to be useful especially in the early stages of the PDP (question 3.2). Participants also mentioned some of the risks related to the use of (virtual) personas. For instance, it was noted that 'we [designers] should not presume to know everything about a user', and that the focus on personas sometimes distracted from the actual use case (see answers to question 3.5).

The insights gained during the session primarily include refined or new product ideas for the use case of the session. A total of 13 new functions and requirements was identified during the session ${ }^{2}$. In addition to the identification and generation of new functions and requirements however, the application also provided insights into how end-users would perceive the new product concepts. It helped participating designers with discussing the concept's feasibility from the perspective of a particular persona. The results of the evaluation indicate that participants expect 'Jim' to accept, like and use the application, while 'Stanley' will probably not use it. Furthermore, the consequences of not using the new product concept were discussed explicitly in Stanley's scenario, as illustrated by the following fragment:

\footnotetext{
${ }^{2}$ The exact results of the session (i.e. the resulting scenarios, functions and requirements) are not included in this thesis because of confidentiality reasons.
} 


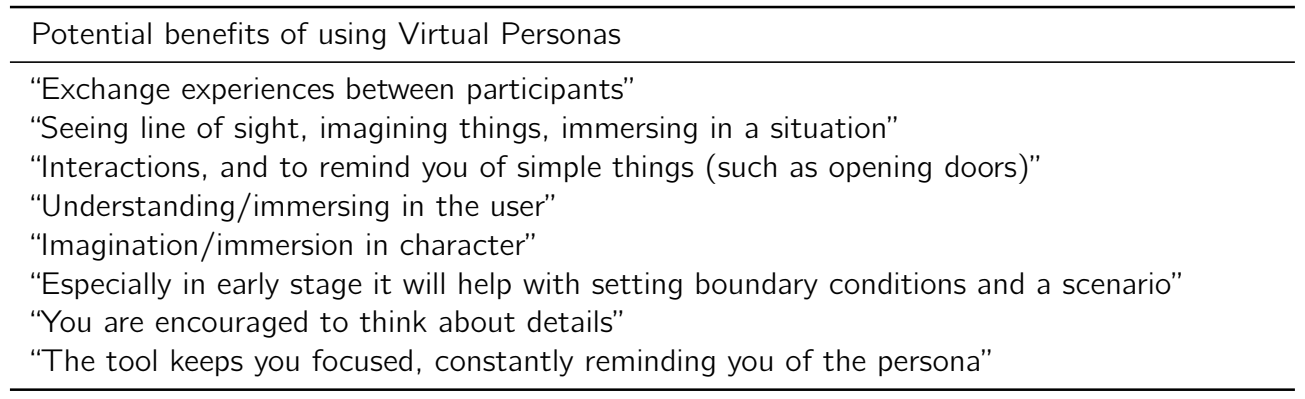

Table 5.1 Potential benefits identified during the application validation session.

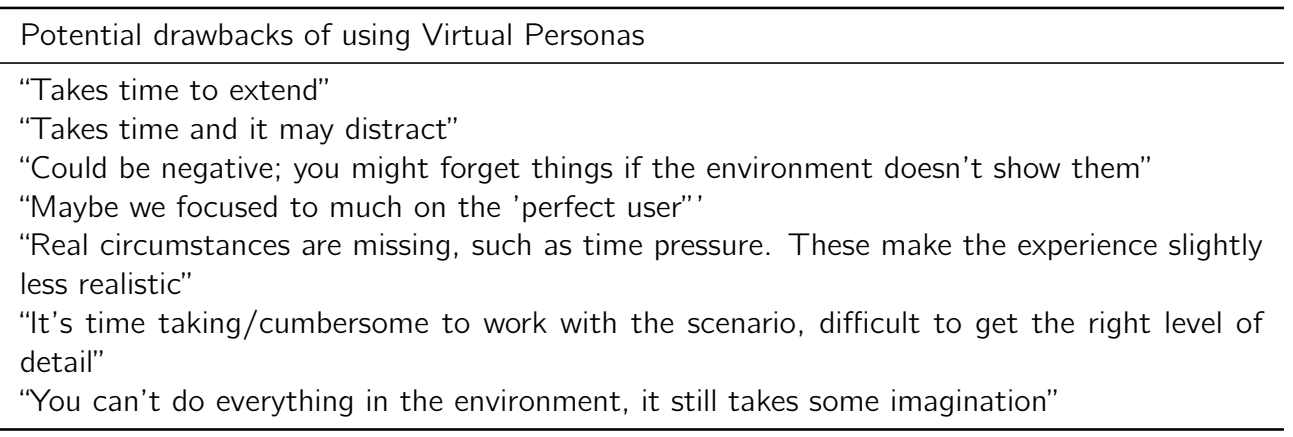

Table 5.2 Potential drawbacks identified during the application validation session.

"Stanley has to do the truck administration manually, from his driver seat. This takes a bit more time than doing it digitally, and it's not automatically updated with the home office. [...] He will give his wife a call to say he won't be eating at home. He uses his own phone for this." (See appendix B.1.2)

This is also reflected by some of the answers to question 2.1, in which participants reason from the perspective of the persona when asked about the expected use of the product concept: "Eventually yes, because it will make his job easier. The threshold is high, and he will only use a small part of all functions/features", and "Yes, he grew up with technology like this en is very capable of using them.".

The answers to questions 1.1, 1.2 and 1.3 (see appendix B.1.2) indicate that although all participants are familiar with personas in general, and are aware of the existence of personas within the company, only 2 of the 8 participants actually have (limited) experience with using them in practice. Consequently, it is difficult to explicitly describe the added value of Virtual Personas. However the answers to subsequent questions provide insights in the advantages and drawbacks of using Virtual Personas.

As listed in table 5.1, the participants indicated that the added value of the virtual environment is that helps with imagining use situations and with explaining and understanding user-product relations. The environment sometimes reminds them of small details or very 'standard' tasks or actions that would otherwise have been overlooked. The validation session also identified several potential drawbacks, as listed in table 5.2. The use of the virtual environment 
may sometimes distract designers from a discussion. Furthermore, it lacks a good simulation of 'time pressure' (e.g. in real-life truck drivers are always trying to reach a destination in time, while the application did not include such aspects), and similar aspects may be forgotten if there are no 'triggers' present in the virtual environment. For instance, in this session objects like a coffee mug and a sandwich triggered designers to think about lunch, which may have been overlooked if the virtual sandwich and mug were not included in the environment. Lastly, the development and extension of the virtual environment is time consuming.

Throughout the session, there were interactions between the group of designers, the tool operator, the virtual environment and the list of tasks and events. In the first few minutes of each session designers systematically picked an item from the list of tasks and acted this out in the virtual environment. As the session advanced, most of the time was spent on group brainstorms and discussions, occasionally fed by information from the virtual environments or events from the list. Here the virtual environment acts as a source of inspiration and reflection for the designers, but does not interfere with the group discussions. Another important observation is that the designers often talked and pointed to the virtual environment rather than the tool operator. This shows that controlling the personas by means of an operator does not negatively affect the interaction between the designers and the virtual environment. It does however rely on a properly trained operator who understands the intentions of designers as well as the capabilities of the tool.

\subsection{Deployment}

Allowing the designers to realise the Virtual Application requires the availability of appropriate preparational tools as well as execution tools, as explained in chapter 3. In the previous case study the tool selection focused on preparational tools because the execution tools were available off the shelf. In the current case study however, it is more difficult to assess the accessibility of both the preparational tools and the execution tools.

In order to investigate both types of tools, a tool selection session involving five participants from the company was organised. The session established the requirements regarding the preperational tools, and subsequently identified execution tools that fit these requirements.

\subsubsection{Tool selection}

\section{Preparation tools}

The researcher identified four application elements that need to be prepared prior to using the Virtual Persona application, as illustrated in figure 5.7.

Based on their experience with the Virtual Persona application (gained during the validation session) the participants were asked to describe criteria for selecting appropriate tools for the realisation of the application. To support this process, the realisation of the individual application element was analysed by answering four questions for each of the following elements, using the form included in appendix B.2.1.

1. Frequency - How often should this application element be created or modified? For instance, should it be created once and never modified, or do you expect it to be modified for each Virtual Persona session?

2. Source - What source would you use for this application element? Would it be imported from internal or external model repositories, would you create a new model, or would a 

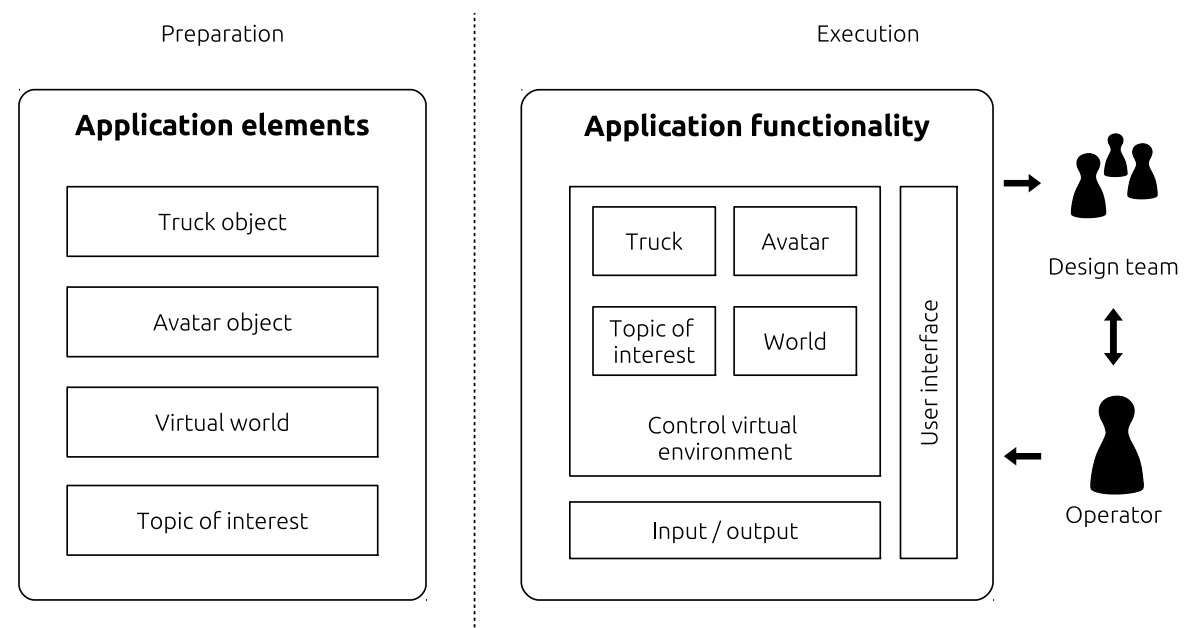

Figure 5.7 Desired functionality for tools.

static built-in model be sufficient for this element?

3. Allocation - Who would be responsible for providing this application element? Can it be allocated to a certain department or person within the company's PDP, or does it require additional skills or resources?

4. Criteria - What are the most relevant criteria for this application element? Does the application element require a high level of detail, is it constrained by time or financial budgets?

The following insights are based on the form results (see appendix B.2.2) and an accompanying discussion between the participants and the researcher.

Firstly, it was found that the participants agreed that both the avatars and the virtual world do not need to be modified frequently. For the virtual world it would be sufficient to build one world that includes various traffic circumstances (e.g. urban and highway). An additional library of world elements (e.g. houses, roads, etc.) could be used to extend or modify the world when needed. Furthermore, the participants indicated that the virtual personas should only be created once in order to 'get to know them, and remember them'. A set of three to five personas is expected to be sufficient. The primary criterion for the virtual personas is that they support a wide range of actions and manipulations, similar to what was used in the application prototype (i.e. the avatar should be able to walk, sit down, take a driving position, etc.).

The topic of interest generally needs to be created or modified for each session, but the exact frequency depends on the type of product that is being investigated. For instance, the virtual persona application can be used to evaluate the layout of an entire truck cabin, which would not require the topic of interest (the cabin model) to be modified for each session. More specific topics of interest, such as new in-vehicle information systems or a new lighting concepts, require specific models to be created for each session. The virtual models of the topic of interest can generally be created by the design department within the company as the required skills 
and tools are already available. The most important criteria for these models are that they are to be sufficiently interactive or functional to be used in a scenario.

Although the application validation session used simplified models of the company's trucks, the designers indicated that the ability to directly use CAD models for truck objects in the Virtual Persona application is an important criterion. This would not only save time (as models can be imported directly from an existing database) but also provide more accurate representations of distances between the driver seat and the dashboard, or the height of the cabin. The preparation of this application element can be done by staff from the engineering department who already have the required tools and skills.

With respect to the realisation of application elements, it is concluded that the primary challenge lies in creating the avatars. The other elements, including the truck, the topic of interest and the virtual world can be realised using tools and skills already available in the company's PDP.

\section{Execution tools}

Having established criteria for preparing the primary elements of the virtual persona application, the researcher introduced four directions of software tools that provide the desired functionality to various extends.

1. Custom software - Custom software, possibly based on the prototype developed for the application validation, can be developed. An important drawback is that the resulting tool will be very application specific, and requires a significant initial investment.

2. PLM/CAD extensions - CAD plugins such as JACK ${ }^{3}$ offer a detailed human model, originally intended for ergonomic analyses. Using such plugins, the Virtual Persona application could be realised within the company's existing CAD platform. The main drawback of this direction is that CAD applications typically lack options for including the context (e.g. the virtual world).

3. Truck driving games - Truck simulation games provide an off the shelf realistic driving experience. The simulations allow customisation of truck models and world settings. The primary disadvantage of this direction is that avatar interactions are usually less detailed, as the focus of these simulations is on driving the truck.

4. Virtual world applications - Virtual world applications, such as OpenSim or Wonderland, provide collaborative virtual environments in which avatars can be moved around, objects can be imported and behaviour or events can be defined programmatically. As such it provides a similar basis as the current prototype. The primary drawback of this direction is that the visual quality of the virtual world as well as the interactions between avatars and objects are usually low.

In a subsequent discussion, the researcher aimed to relate the requirements with respect to specific application elements to the available execution tool directions. For example, if visual realism is an important criterion for e.g. the virtual truck models, an execution tool such as the virtual world application is less feasible as it does not support the rendering of highly detailed 3D models.

The execution tools were introduced by showing screenshots and by presenting their key characteristics, benefits and drawbacks. Based on this information, the participating designers

\footnotetext{
${ }^{3} \mathrm{~A}$ virtual dummy, see http://plm.automation.siemens.com/en_us/products/tecnomatix/assembly_planning/jack
} 
were able to assess the tools. It was generally agreed that the truck driving game direction and the virtual world direction did not fit some of the criteria that were defined earlier. The driving game provides a visually rich virtual world but lacks support for importing custom models and does not allow designers to implement specific interactions. The virtual world direction provides a flexible and open development environment, but has limitations with respect to interactions between avatars and specific products, such as a truck or its interior.

While discussing the PLM/CAD direction and the custom software direction, the designers indicated a preference for the PLM/CAD direction. The primary reason for this is that the company aims to create and maintain a smooth tool chain throughout their PDP. Therefore, a custom built tool to support the virtual persona application is less desirable than extending their existing CAD and PLM systems with similar functionality. Existing extensions, such as the JACK virtual dummy, could be a starting point for realising virtual personas in their current tool chain. Furthermore, the use of these tools potentially allows the application to facilitate other design tasks, for instance concerning ergonomics, as well.

It was concluded that, if the company would implement the Virtual Persona application, it would use their current PLM/CAD system as a basis. It is expected that the system's limitations, such as the lack of context representation and the lack of real-time interactions, can be solved with additional extensions and/or other customisations to the software. This would result in an application that integrates with the company's existing tool chain and potentially supports other design tasks as well.

\subsubsection{Tool evaluation}

While it was concluded that PLM/CAD tools would be used for the preparation and execution of the Virtual Persona application, the actual implementation of these tools is outside the scope of the research. However, a sufficiently effective and usable tool has to be provided to the company involved in the case study in order to gather insights into the feasibility of realising the application within the company. To achieve this, it was decided to provide the company with a substitute tool that provides the required functionality for preparing and executing the application.

\section{The Virtual Persona tool}

The Virtual Persona Tool is based on Blender, a 3D development environment that was also used to create the application prototype. The tool supports the preparation of the Virtual Persona application by providing a virtual world into which various static (e.g. roads and houses) or interactive objects (e.g. avatars or trucks) can be imported (see figure 5.8). Template versions of primary application elements, such as avatars, trucks and virtual cameras, have been created as modules that can easily be imported into the virtual world without additional programming or modelling. As indicated during the tool selection step, designers would like to have control of the virtual personas in terms of movements, actions and positioning. The avatar templates provided by the Virtual Persona tool support automatic and manual interactions (as described in section 5.3), and allows designers to add custom animated behaviour, such as 'making a phone call' or 'reading a book', which can be activated during the execution of the application.

To further support the evaluation of the Virtual Persona tool, the company is provided with documentation on how to use the Virtual Persona tool. The documentation covers development aspects (e.g. how to create new models or behaviour) as well as use aspects (e.g. how to change camera viewpoints or how to control virtual personas). The documentation is placed on 


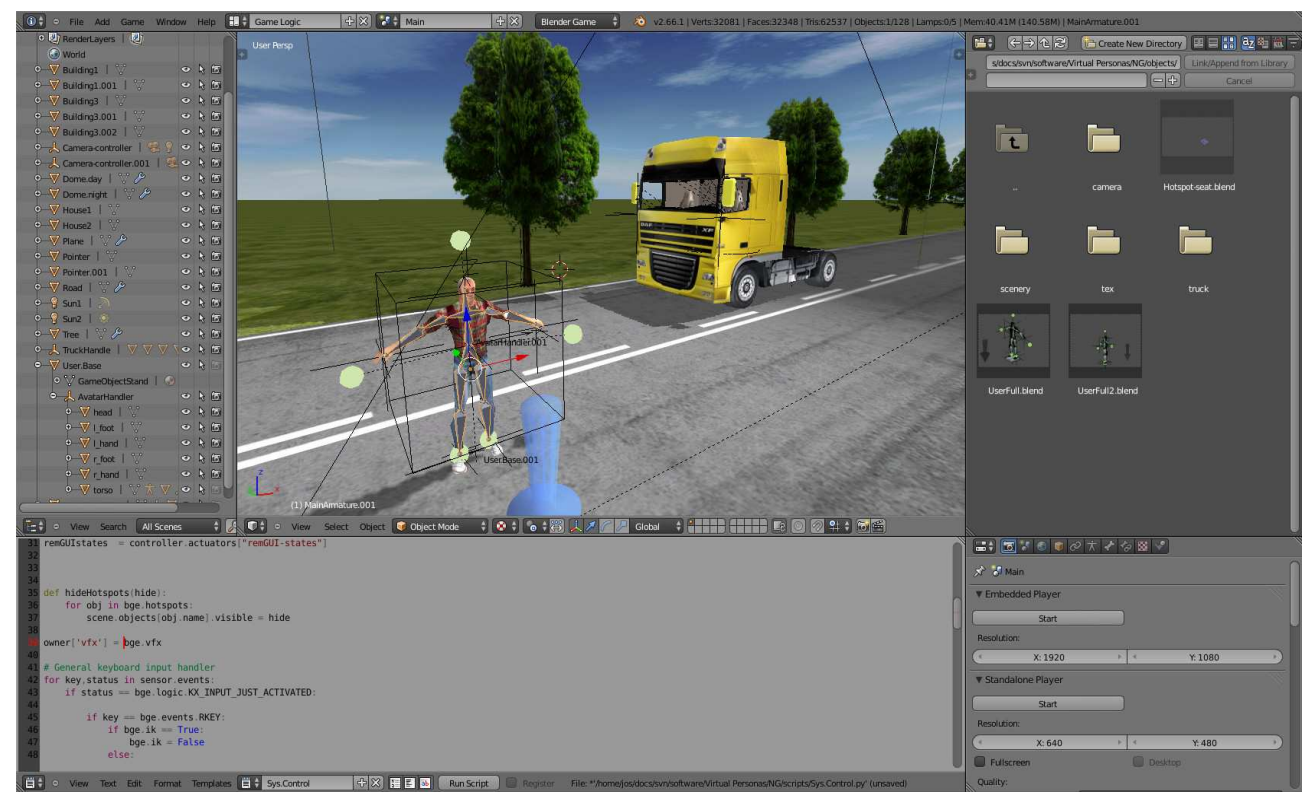

Figure 5.8 This screenshot shows the Virtual Persona environment loaded in Blender. Blender provides access to persona textures, the scripting code behind interactive objects, and facilitates importing additional virtual objects.

a website and includes several extensive video tutorials that aim to guide the designers through various tasks required for the development and use of the application.

\section{Evaluation results}

The evaluation of the Virtual Persona tool and its documentation is based on feedback provided by the company after a period of time in which the company was given the opportunity to explore the tool.

In general it was found that the Virtual Persona tool provides a structured and modular way of creating custom virtual worlds and personas. Interestingly, the designers found that the direct access to the source (code as well as 3D models) of the virtual environment also triggers the need for making additional modifications, such as:

1. Writing custom scripts and animations

2. Importing detailed human body models

3. Importing existing CAD models

4. Modifying the user interface

While such modifications are supported by the Blender development environment on which the Virtual Persona tool is based, this is quite a big step for designers to make as it requires 
knowledge about programming and 3D modelling, but also a proper understanding of the structure of the virtual world that is used in the application.

Although the Virtual Persona tool and the supporting Blender development environment are considered too complex for realising the Virtual Persona application, the company indicated that it is worth investing in getting to know the tool better if this also allows for the realisation of other applications.

\subsection{Conclusion}

The Virtual Persona application that was developed in this case study illustrates how VR techniques can be used to support an existing design method. In this case, the company was already familiar with personas, but found it difficult to apply them in the PDP. The Virtual Persona application addresses this problem by allowing designers to act out use scenarios featuring personas that are represented by virtual avatars. As such the application allows the designers to temporarily step into the shoes of a specific persona, and generate or evaluate product concepts from this specific perspective.

Throughout the case study there have been two challenges that affected the implementation of the case study specification method. Firstly, the initial exploration and development phases of the case study required some modifications in order to cope with the company's focus on a very specific form of $\mathrm{VR}$, namely driving simulations. Even before starting the case study there was quite a clear view of a desired application with this type of technology. Although this focus makes sense as the company is involved in the development of automotive products, from a research perspective it was important to explore a broader range of possibilities and to focus on user involvement. The VR exploration workshop therefore explicitly attempted to broaden the company's scope with respect to available VR technologies and their applications, which eventually led to the Virtual Persona application. Secondly, despite a successful validation of the Virtual Persona application, it turned out to be difficult to refine the requirements with respect to the preparation and execution tools needed for the realisation of the application. There are two factors that may have affected this challenge:

- The application description was not defined sufficiently in the initial stages of the case study. Although the exploration workshop and the subsequent application refinement session described the use of personas and the expected added value of virtual personas, case study participants who were not present at the refinement session may have had different expectations of the application. As a result, during the prototyping and application validation stages the application extended to various other applications, such as the analysis of ergonomic aspects in the virtual cabin, and the use of the environment to conduct low-fidelity driving simulations.

- Because of the complexity of the product that is being developed the company relies heavily on CAD and PLM systems for the generation and management of product data. Although it makes sense to also use these tools for the preparation and/or execution of the Virtual Persona application, it was found that the use of these detailed tools (and models) can also cause participants to get lost in details instead of being able to oversee use situations.

Because of these challenges, the case study eventually resulted in an application description and tool selection that represent a compromise between a truly early stage design tool and a more detailed validation and evaluation tool. It was shown that the application, even when using 
low fidelity models and technologies, effectively facilitates idea generation sessions involving personas. The subsequent selection of tools, however, led to a more high-fidelity tool chain, following the specific requirements of the company involved in the case study. Although these tools do provide the functionality required to realise the application, it is expected that the application can also be realised using less sophisticated means. This should be taken into account when translating the application to the other companies, during the cross-company evaluation sessions. 


\section{$6 \quad$ Case Study 3}

The third case study involves the development of the Virtual Annotation application. This VR application enables multidisciplinary design teams to collaboratively review and annotate product concepts in a very early stage of the development process. The visualisation of the product and its use context supports in identifying, evaluating or validating initial product requirements, but can also help with brainstorming about new product functionality. The insights gathered during these sessions are documented automatically and can be shared with other stakeholders in the development process.

\subsection{Introduction}

The company involved in case study 3 develops industrial food processing machines for producing dough or pastry based products. The machines (also referred to as 'units') are delivered in a line configuration that meets the specifications of a particular production process prescribed by bakeries.

The company's PDP is primarily platform driven, relying on standardised units to provide common functionality (e.g. slicing, pressing or rolling). When customers require specific functionality, a standard unit is customised to meet these requirements. This approach requires collaboration between a technical sales department which is in charge of deciding whether or not a customised machine is required, a research \& development department which is in charge of designing the required customisations, and an engineering department which is in charge of implementing the customisations.

One of the challenges the company faces is the translation of client needs (e.g. requirements with respect to the bakery's production process) into specifications for a food processing machine. This is an area where the company expects UCD, possibly supported by VR technologies, could help.

\section{Chapter outline}

Section 6.2 further explores the company's challenges and expectations with respect to VR and UCD and describes the results of the VR exploration workshop. The workshop leads to the definition of the Virtual Annotation application, of which a functional prototype is presented in section 6.3. Section 6.4 presents an overview of means that are available to realise this application within the company. 


\subsection{Exploration}

The Exploration phase identifies potentially useful applications of VR within the PDP of the company and involves a kick-off meeting and an exploration workshop.

\subsubsection{Kick-off}

The kick-off meeting is a preliminary meeting with a small team of company participants, in which the researcher outlines the approach of the current case study and briefly summarises the results of the previous two case studies. Furthermore, based on insights gathered during the interviews and site visits (see section 2.3), the researcher discusses several challenges and bottlenecks in the company's current PDP that could serve as a focus area. The discussion results in three topics that the company considers relevant for further investigation in the case study.

1. Transfer field knowledge - It is important for engineers to understand how their machines are used in practice. However, without sending engineers into the field (which is time consuming), it is difficult to capture and transfer the required field knowledge and experience into the engineering and $R \& D$ departments.

2. Tracking customer requirements - Since the number of stakeholders (e.g. buyers, bakers and operators) involved throughout the development process is quite high, it is difficult to identify and track customer requirements. In addition to capturing and tracking customer requirements, this information needs to be transferred to appropriate departments internally.

3. Communicating USP's - To support the company's sales process, it is important that the unique selling points of the machines offered by the company are communicated effectively.

These topics serve as a starting point for the exploration workshop, in which VR applications are identified that address these (or additional) topics.

\subsubsection{VR exploration workshop}

As explained in chapter 3 , in the exploration workshop visual storyboards are used to let designers create their own representations of desired VR applications. The exploration workshop for this case study involved seven participants (one R\&D project leader, a R\&D engineer, two sales engineers, the product manager, the head of engineering, and one member of the product management and calculation department) and took place at the company's premises. Prior to organising the workshop, example storyboards were created based on the outcome of the kick-off meeting. The next subsection further elaborates on these example storyboards.

\section{Preparation}

The preparation of the exploration workshop consists of creating three animated storyboards that serve as a starting point for the individual storyboards created by workshop participants. The topics of the example storyboards, listed in table 6.1, are based on the results of the kick-off meeting. 


\begin{tabular}{ll}
\hline Activity & VR Application \\
\hline Transfer field knowledge & An application that captures a specific use context (such \\
as a client's factory) and enables designers to virtually \\
explore this use context. The application aims to pro- \\
vide the company's engineers and designers with 'field \\
knowledge' without having to go out there themselves. \\
Tracking customer requirements & $\begin{array}{l}\text { An augmented reality application is used to provide } \\
\text { clients with a virtual preview of the products they ordered } \\
\text { in their own use context. The demonstration storyboard } \\
\text { shows a mobile augmented reality solution that allows } \\
\text { clients and designers to collaboratively preview or review } \\
\text { a virtual product prior to the engineering stages. } \\
\text { This application supports communication between future } \\
\text { clients and the sales engineers of the company by allowing } \\
\text { future clients to virtually compose and review a machine } \\
\text { configuration before actually buying it. The application } \\
\text { supports the sales process but also provides designers } \\
\text { and engineers with insights into client requirements and } \\
\text { specifications. }\end{array}$ \\
\hline
\end{tabular}

Table 6.1 VR applications proposed for design activities

As in the first case study, the animations intentionally have a low visual fidelity to reflect the explorative and inspirational nature of the storyboards. The animations are recorded using the stop-motion technique, resulting in a particularly choppy and crude animation. Furthermore, the use of toy actors and paper props contributes to the experimental and unfinished nature of the storyboards, giving workshop participants the idea that there is room for improvement, exploration and modifications. In spite of the low fidelity, the storyboards still effectively communicate the nature of the VR application, its purpose in the PDP and the practical context in which it is used.

\section{Proceedings}

As in the other exploration workshops, it took a while for the participants to get used to working with the storyboard. Most of the individual storyboards were strongly related to the creator's department; there were two sales oriented storyboards using VR to support communication with customers, three engineering storyboards that generally focused on team communication and two other individual storyboards that addressed the company's PDP on an organisational level (see figure 6.1a).

Based on experiences in case studies 1 and 2, an extra discussion between the individual storyboard round and the group storyboard round was held. During these discussions, the facilitator was able to identify similarities and differences between the storyboards. With these findings the individual storyboards were grouped into three groups with specific application themes. Because of the central round of discussions held prior to forming the groups, the participants could immediately start creating a group storyboard.

In the final stage of the workshop, the participants presented their storyboards to the group. The presentations depict how, when, why and by whom VR is used to facilitate the 


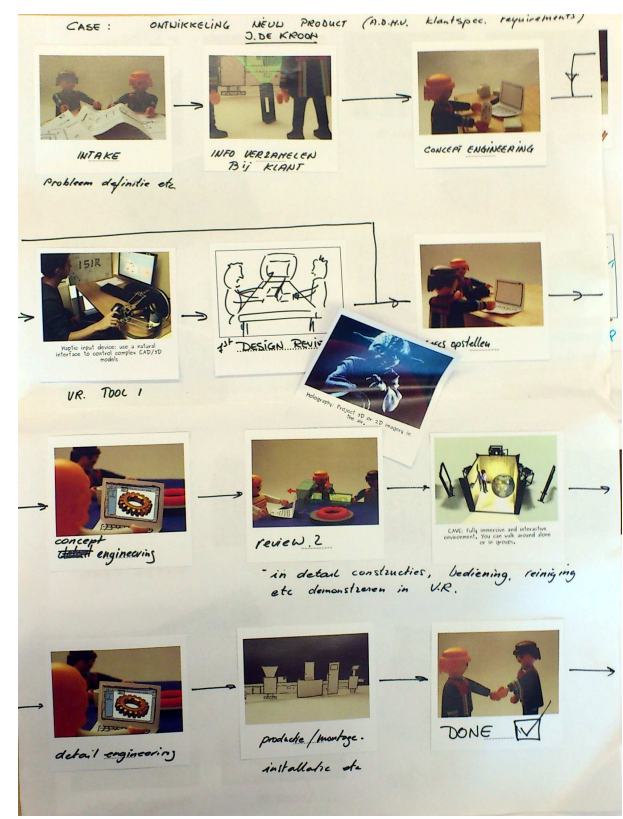

a. An individidual storyboard featuring various forms of VR technologies.

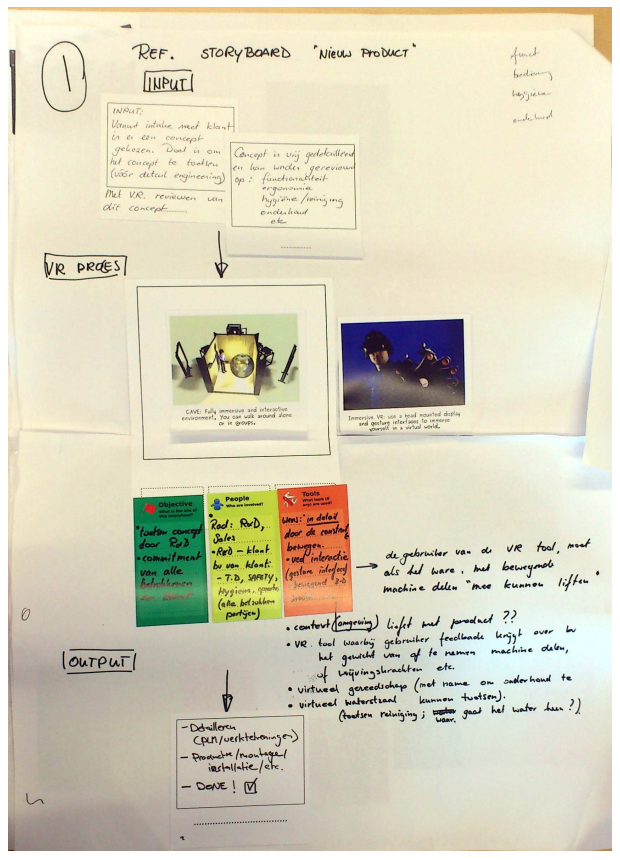

b. A group storyboard showing more detailed description of a VR application.

Figure 6.1 Storyboards resulting from the exploration workshop.

company's PDP (see figure 6.1b). Overall, the resulting storyboards show quite a diverse range of applications as well as technologies. The engineering oriented storyboards tend to rely on detailed and high fidelity visualisation techniques, such as CAVES and CAD models, while the sales oriented storyboards propose to use mobile and flexible solutions such as augmented reality.

\section{Results}

The group workshop generated three potential application directions, as illustrated in figure 6.2;

1. Theme 1: Group Review of R\&D Work - Storyboard 1 intends to use VR to facilitate communication between the R\&D department and other stakeholders. This application takes place after sales and sales engineering, and involves discussions and reviews of relatively detailed subjects. The storyboard explicitly mentions aspects such as cleaning, maintenance and use to be included in these reviews because they are currently unsupported by CAD applications.

2. Theme 2: Virtual Machine Configurator - Storyboard 2 introduces a virtual machine line configurator, which was mentioned in several individual storyboards. This sales oriented VR application allows customers to compose a machine line (possibly combined with 3rd party machines or black boxes) made up of standard units provided by a virtual model library. The technology used in the application should support collaboration, similar to 


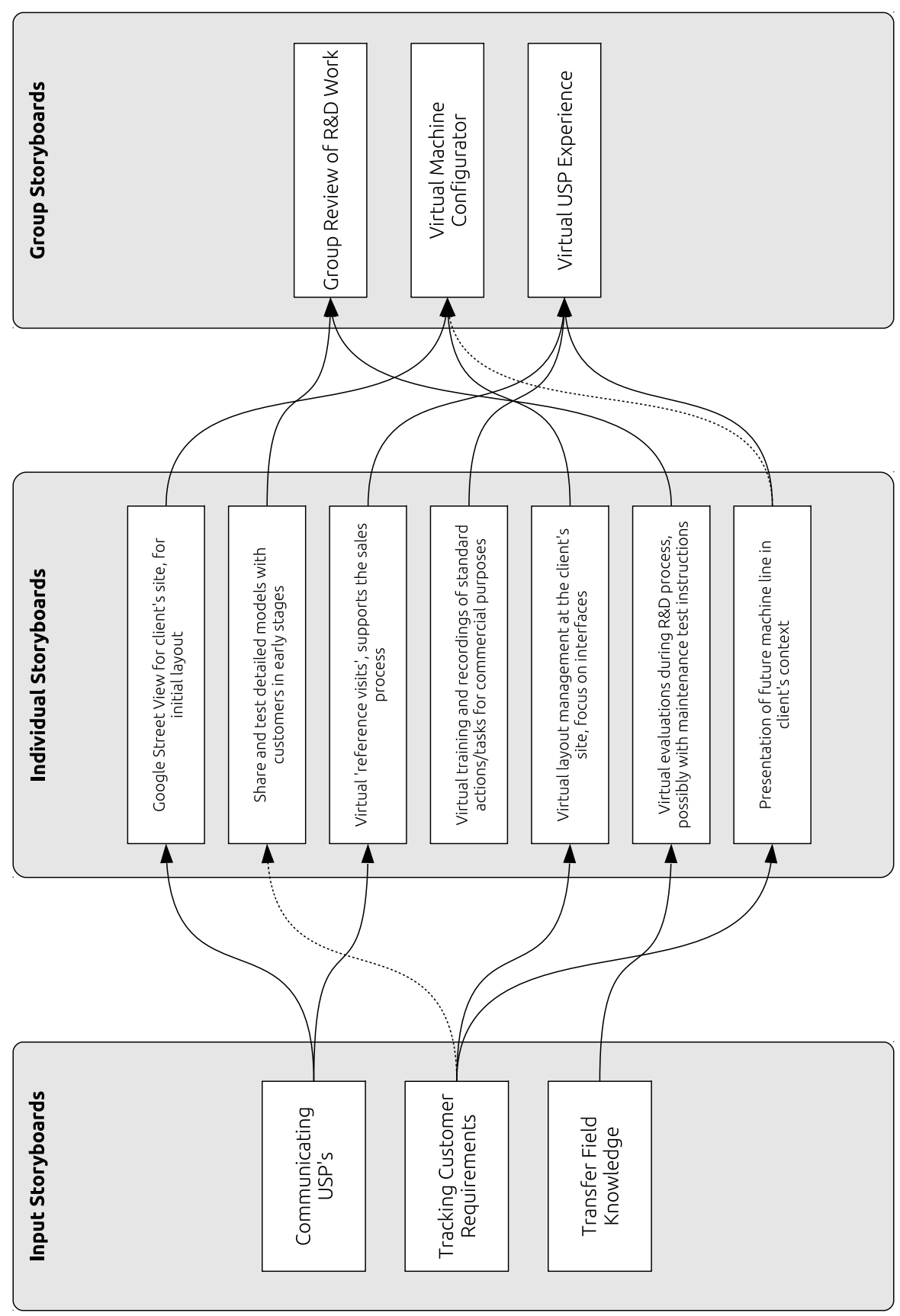

Figure 6.2 Visualisation of individual and group storyboards. 
e.g. multi-touch table displays or AR tables. The application gives the customers an idea of the final situation and allows them (and sales engineers) to pinpoint the units that need to be customised (which can be used as input for R\&D). Aspects such as layout management (making sure a machine will fit) could also be taken into account in this application.

3. Theme 3: Virtual USP experience - According to Group Storyboard 3 high-end forms of VR such as a CAVE setup, can be used to communicate the company's unique selling points (USP's) to potential customers. It is currently quite difficult to explain the functionality of a specific part, let alone its competitive advantages. Storyboard 3 proposes to let clients virtually experience the USP's in VR. Compared to Storyboard 2, this application envisions a more 'hands-on' experience with some of the company's unique selling points, for instance showing how fast or easily a particular component can be replaced.

Themes 2 and 3 contribute to convincing a potential customer to buy the company's products and to giving the customer a more complete view on the future situation by letting them configure the line themselves. Theme 3 in particular is directed to output only, meaning that it primarily uses VR to present existing machines to potential buyers. While it may elicit some feedback, the primary aim is to inform (or convince) the user. In addition, the users involved in this proposed solution will usually be decision makers rather than actual end-users of the machines. Theme 2 describes a slightly more design and end-user oriented direction as it lets customers configure a machine line, and, after a deal has been made, additional stakeholders (operators, engineers) can be involved in reviewing the prospect machine layout.

Contrary to themes 2 and 3, theme 1 directly addresses product development activities by facilitating communication between R\&D and other stakeholders. The stakeholders can be internal (i.e. the sales department, engineering department or the manufacturing department) or external (e.g. machine buyers, maintenance engineers or operators). By improving the communication between R\&D and these internal and external stakeholders, the company expects to establish a better (and shared) understanding of what needs to be and is being developed, and identify design errors in an earlier stage, consequently shortening the product development cycles.

It was concluded that theme 1 provides the most relevant theme for further investigation in the case study. Therefore, theme 1 will form the starting point for the development of a VR application.

\subsubsection{Application description}

Following the outcomes of the exploration workshop, the VR application that will be developed in this case study will suport R\&D activities by facilitating multidisciplinary design reviews in the early stages of the PDP. VR will be used to provide stakeholders with a holistic view on the product that is being developed, in the early stage of the PDP.

The envisioned application consists of two primary components, namely a preliminary 3D representation of the product that is being developed in its use context, and a structured set of requirements and specifications that is being created as participants review and annotate the 3D representation. Regardless of their professional background, all group members should be able to manipulate the 3D representation (e.g. change viewpoints, move around objects, etc.) and edit the requirements and specifications. This way the application will facilitate discussions that lead to a shared and thorough definition of product requirements and specifications. 


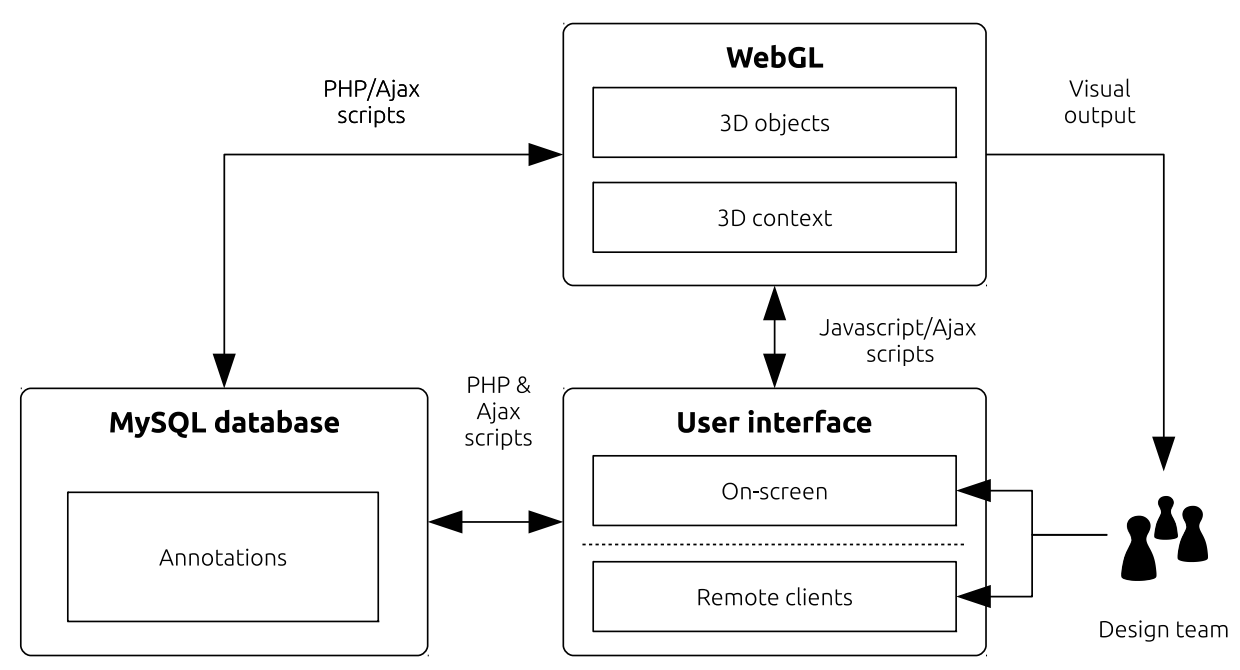

Figure 6.3 Diagram of the Virtual Annotation application prototype.

Applications similar to the one outlined in this section can be found in the domain of Computer Supported Collaborative Work. This domain is concerned with developing software for capturing and structuring knowledge in collaborative settings. Lenne et al. (2009) provide an overview of virtual annotation applications that have been developed so far. One of the applications mentioned is Redliner which later led to a follow-up application called Space Pen, further described by Jung et al. (2002). The Redliner and Space Pen applications provide the functionality desired in the current case study, namely the annotation of $3 \mathrm{D}$ models and the structuring of the resulting knowledge.

While existing applications such as Redliner and Space Pen provide the required functionality, their implementations are no longer available because of outdated supporting technologies such as Java3D. Furthermore, the evaluation of the applications lead to several recommendations that can be used for a new implementation of this application. For example, Jung et al. (2002) mention the need for simplified 3D navigation, improved organisation of comments, drawing annotations directly on the 3D environment and direct manipulation of 3D models. Lastly, as the current application aims to support design team collaboration, a new implementation could explore possibilities to facilitate multi-user input through new forms of input devices.

\subsection{Development}

This section describes the development of a functional prototype of the Virtual Annotation application. The resulting functional prototype is used in a test session to validate the application. 


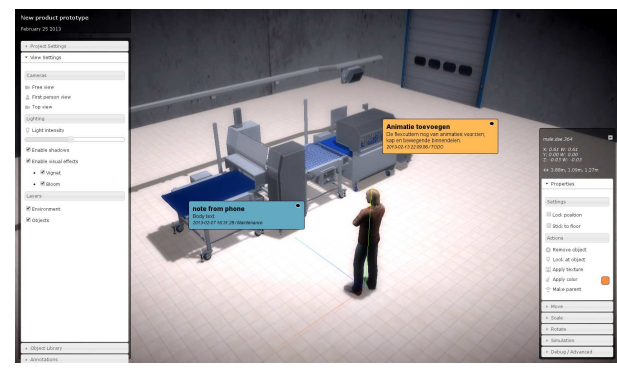

a. Annotations can be connected to specific parts of the $3 d$ scene.

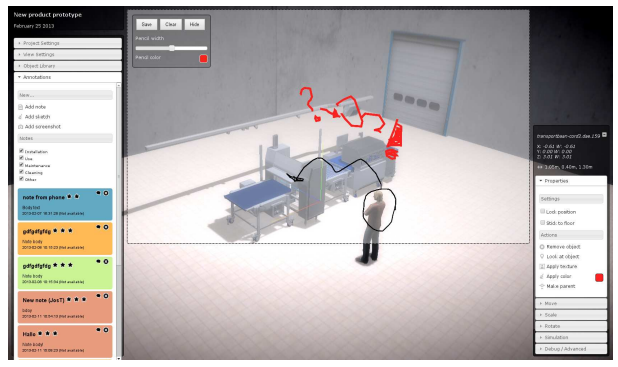

b. The list on the left shows a structured overview of annotations. The $3 d$ scene shows a free-hand sketch annotation.

Figure 6.4 Screenshots of the Virtual Annotation application prototype.

\subsubsection{Application prototype}

The Virtual Annotation application prototype developed for this case study makes use of several established technologies, as illustrated in figure 6.3. The basis of the application uses WebGL ${ }^{1}$ for hardware accelerated $3 \mathrm{D}$ rendering. This makes it easier for the researcher to share the prototype with designers from the company, for instance for previewing initial prototype versions. An interface layer on top of WebGL provides a user interface and interactions for model manipulating, importing and exporting models, changing viewpoints and adding annotations. An annotation framework based on a MySQL database is used to store and structure the annotations created in the application.

The desired functionality of the application outlined in section 6.2.3 is divided into three tasks that the application prototype supports.

1. Create a coarse product model - The starting point of this process is the definition of the concept 'on paper', for instance a preliminary product description or a product opportunity identified by marketing activities. The first step is to form a coarse 3D representation of this product concept, consisting of an external design and possibly parts that represent the core functions of the product. This step can be achieved with existing tools, but is also supported by the VR application.

2. Review and annotate the product model - Having created this model, the second step consists of a collaborative product review facilitated by the VR application. The application allows a design team (possibly including external stakeholders) to review, discuss and modify the coarse 3D representation of the product, and annotate specific parts of it through text and sketches (see figure 6.4b).

3. Document and share the gathered knowledge - The third task that is supported by the application is the documentation of all annotations, discussions and feedback generated during the collaborative session. The application automatically collects this data and presents it in a customisable report.

To investigate how to support interaction between the participating designers and the virtual

\footnotetext{
${ }^{1} \mathrm{An}$ implementation of the OpenGL graphics library for online applications, see http://www.khronos.org/webgl/
} 


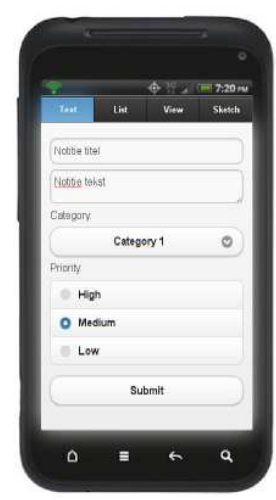

a. Smartphone client showing the text annotation function.

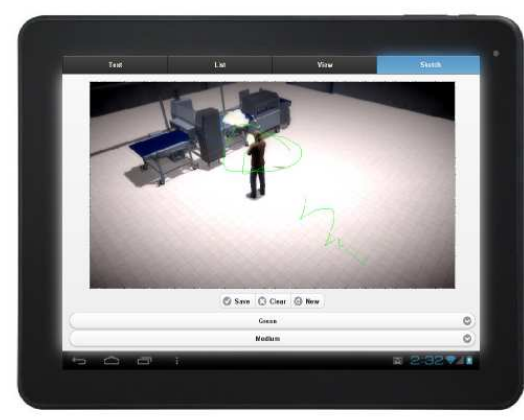

b. Tablet client showing the sketch annotation function.

Figure 6.5 Remote client user interfaces.

environment, the interaction with the Virtual Annotation application is implemented as a design parameter. The application supports two forms of interaction;

- Moderated - A dedicated moderator, who is not involved as a designer in the session itself, takes care of inserting and adding annotations to the model. While discussing or brainstorming, session participants can ask the moderator to add a specific annotation. The moderator also controls the viewpoints and modifies the models or layout as needed.

- Individual - All session participants have a personal input device to add annotations and to navigate through the virtual environment. This still allows for group discussions and brainstorming, but also enables participants to write down or sketch their thoughts while other participants are discussing.

To this end, the prototype provides an on-screen user interface for the moderated approach, and allows client devices (e.g. smartphones, tablets or laptops) to connect to the group session (see figure 6.5). In addition to the above two methods, a hybrid form in which both methods of interaction are allowed can also be explored.

\subsubsection{Application validation}

The Virtual Annotation has been validated in a use case within the company. The use case featured the design of a new multi-functional cutting machine referred to as the SuperSlicer. When the case study was taking place, the development of this machine had only reached the stage of paper specification and an initial outline of its physical design. The use case therefore provides a good setting for evaluating the use of the Virtual Annotation application in identifying, evaluating and validating requirements for this new machine.

Prior to executing the review and annotation session, two experienced engineers were asked to create a preliminary 3D model of the SuperSlicer based on initial specifications and sketches. A model of the new product was created in a two-hour session, of which one hour was spend on modelling and one hour on collecting other models for the use context. The engineers used their present CAD tools to create coarse models. To do so, the engineers successfully made a 
mental switch from exact engineering and dimensioning to conceptual drawing and modelling in the familiar CAD application.

\section{Approach}

The application validation consists of two identical sessions in which groups (referred to as group $A$ and $B$ ) of four participants and a project leader (the project leader is present in both sessions) use the Virtual Annotation application to review the SuperSlicer concept. Table C.1 in appendix C. 1 provides a list of group members. Both groups have 1 hour and 15 minutes to complete the session, which also includes a short introductory presentation and demonstration of the application.

Both sessions follow an identical approach;

- Introductory presentation and demonstration (15 min.)

- Application round 1 - With moderator (15 min.)

- Application round 2 - With individual input devices (15 min.)

- Application round 3 - With moderator and input devices (15 min.)

- Filling out an evaluation form (see appendix C.1.1) (10-15 min.)

The group participants were seated near a large screen on which the virtual environment was projected (see figure 6.6). The facilitator was positioned outside this group, but was able to communicate with the participants. The project leader stood in front of the group (near the screen) most of the time.

\section{Proceedings}

The sessions start with an introductory presentation that gives an overview of the aim of the test session, introduces the application and outlines the three rounds. Before using the virtual environment, participants are asked to write down how they would currently approach a design task similar to the one they are given in this session (i.e. to review an early stage concept from the perspective of future end-users). The current approach is compared to the new (VR supported) approach in the post-session evaluation form.

The session involving group A started with an introduction to the SuperSlicer concept by the project leader. Standing in front of the virtual environment, the project leader outlined the functional behaviour of the machine and pointed out specific features such as styling, interaction and dimensions. The project leader also explained that the 3D model is just a coarse model, making clear that the participants should 'look through' glitches in the model or environment (as some of the units in the virtual environment did not correspond to a real-life situation). The discussion within the group was initiated after one of the lids of the SuperSlicer was opened, hitting an overhead cable guide. The participants immediately noticed the problem of the lid hitting the cable guide. After adding this finding to the list of notes, participants continued to various other discussions. The group mainly used text notes to annotate the model. Only 2 sketches were made to clarify specific issues (depicting a tool trolley and the fixation of the SuperSlicer to the floor). After handing out the individual input devices (the session used iPads), the group discussion was disturbed and did not fully recover; the participants spent time getting to know the new user interface and on typing in notes and adding sketches. During 


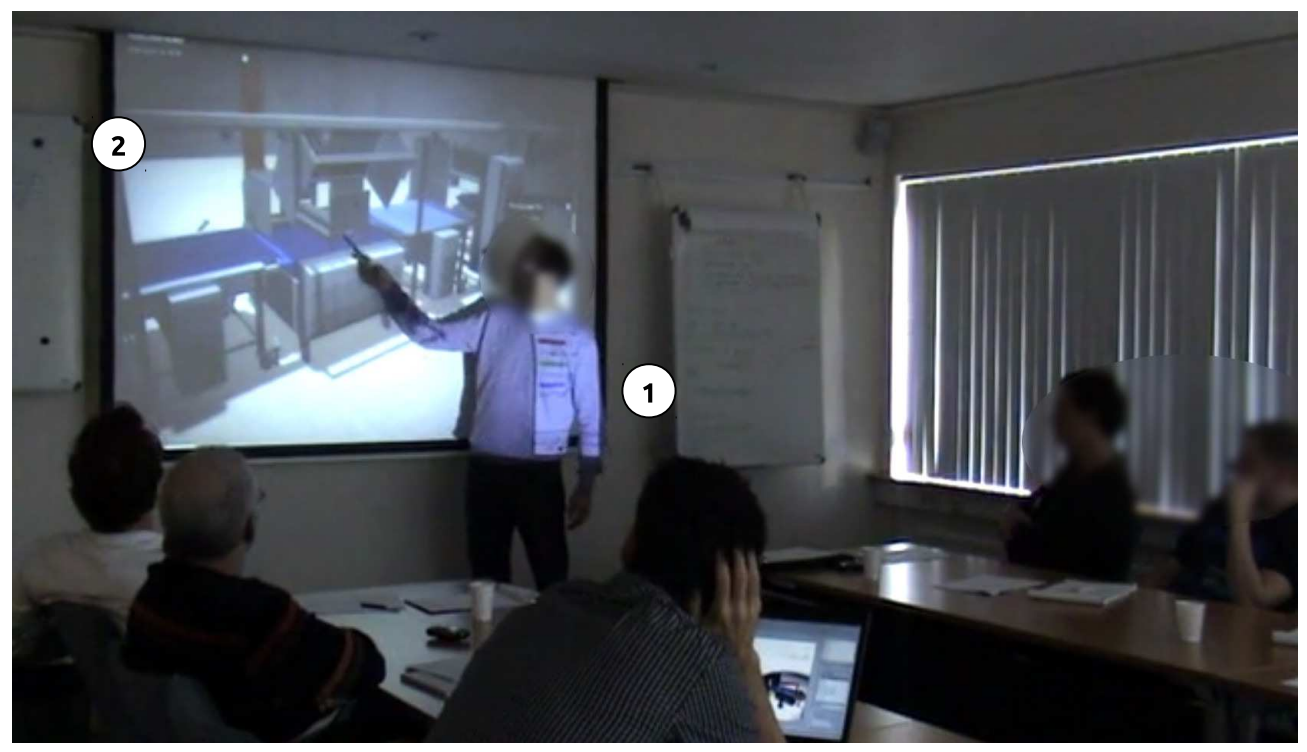

Figure 6.6 The application validation session. Most of the time the project leader (1) stood next to the screen (2) to point out specific aspects of the machine or to explain a particular situation.

the last round, where participants could chose their preferred method of interaction, the group discussion returned, but not to the level of the first round.

Group B also started with an introduction to the SuperSlicer machine given by the project leader. As with group $A$, one of the first things that was discovered was the problem of the lid hitting the overhead cable guides. However the second group also managed to identify several new issues (for instance a more detailed discussion about the fixation of the SuperSlicer to the floor). This can partly be explained by the fact that the findings of the first group were visible in the note database, thus allowing the participants of group B to think of different topics and issues.

Having experienced the first session, the facilitator stressed that it was important to continue the group discussion and not focus all attention on the devices. Consequently, group B was less distracted by the introduction of the individual input devices. While the group discussion continued (sometimes between two or three participants) the remaining participants were able to add notes or sketches in parallel to the discussion.

\section{Results}

The validation sessions covered the review and documentation functionality of the application. Together with the creation of a coarse initial product model (carried out prior to the validation sessions) all three functions of the Virtual Annotation application were tested. Based on an analysis of the video and audio recordings of the sessions and the results of the post-session evaluation (see appendix C.1.2), the following is concluded.

The 3D visualisation of the SuperSlicer in a use context provided an effective communication channel between the project leader and the session participants. Even though most 


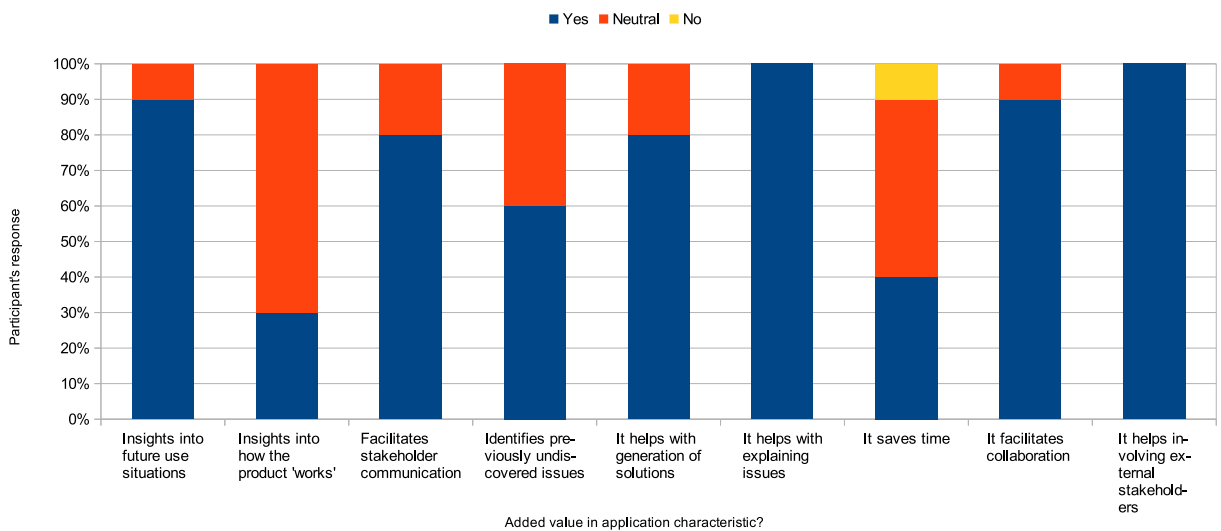

Figure 6.7 Answers to question 1.2 of the post-session evaluation form, asking participants to indicate the areas in which the Virtual Annotation application provides added value.

participants were new to the topic, the project leader was able to quickly introduce the product and trigger several relevant discussions. While the research did not propose a specific structure for the meeting, the project leader started both sessions with a quick introduction of the product. This proved to be a successful exercise during both test sessions. As shown in figure 6.7, similar qualities are considered to be the most important benefits of the Virtual Annotation application, namely the ability to explain or introduce (issues with) product concepts, and the ability to introduce uninformed stakeholders to a (new) product concept. The answers to question 2.1 indicate that visualisation plays an important role in providing this added value. Visualisation helps with quickly understanding the situation and relevant aspects of the model. When comparing the Virtual Annotation application to existing CAD tools, which in principle could also facilitate such sessions, participants indicated that the current application shows a more realistic picture, in spite of the simplified models;

"[...] it establishes and maintains a focus. Participants are triggered by the constant representation of the design." (See appendix C.1.2)

The current application includes shadows and shaded/dirty machine parts, giving it a more realistic appearance. Other functions that CAD tools generally do not provide, include simple dough and water simulation which was also received positively.

Participants also acknowledged the added value of the application's review and annotation functionality. As shown in figure 6.8 the use of notes is preferred over the use of sketches. It is expected that this is partly due to the technical implementation of the sketching function in the application prototype; some features, such as the ability to zoom in/out on a sketch, or the ability to undo a particular action, were not available.

The results of the evaluation forms (questions 2.7 and 2.8) indicate a preference for a moderated discussion form, in which participants can ask a moderator to add annotations. Although 9 out of 10 participants prefer a moderator, the answers to questions 2.7 and 2.8 do not rule out the use of individual input devices. The use of individual input devices requires initial training or instruction before being able to use it as intended. In the validation sessions, 


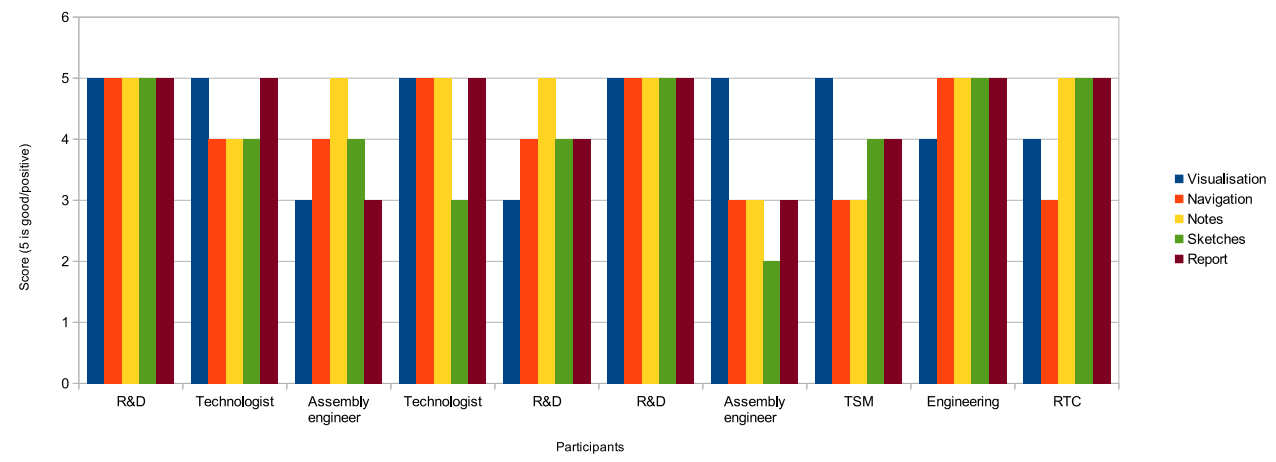

Figure 6.8 The importance of the five core functions of the Virtual Annotation application, as indicated by participants (question 2.2 of the post-session evaluation form)

which did not include extensive instructions about the input devices, the individual input devices blocked the group collaboration because participants needed time to get used to it, and to find out when to use the devices. The session with group B provided the best insights into the use of individual input devices, as this group managed to use the input devices without disturbing group discussions. There were several occurrences of 'parallel discussions'; while the majority of the group is discussing a central topic, one or two other participants can add annotations about completely different topics and come back to this later. A consequence of these parallel discussions, however, is that reviewing the list queue disturbs the current discussion; a facilitator has to ask participants what a particular note is about, and to which part of the model it is related.

The application's documentation feature is considered useful, as shown in figure 6.8. However, the participants did not consider the document to be a 'finalised' specification document but rather a collection of all the ideas, feedback and comments uttered during discussions. Consequently, the documentation feature should capture and structure as much data as possible (i.e. not only the notes and sketches, but also the discussions).

After the validation session, participants mentioned the possibility of using annotations in an asynchronous setting; instead of organising a central meeting, the application could be accessible (e.g. via the web) for a longer period of time and allow designers or engineers to add annotations whenever they think of something. This setup could also facilitate situations in which stakeholders (e.g. designers and customers) are spatially separated. While this is an interesting application that is technically feasible with the current prototype, it is outside the scope of the current research to further investigate.

\subsection{Deployment}

The deployment phase of the case study involves the selection and evaluation of the means for realising the Virtual Annotation application. As explained in chapter 3, these means include preparation tools and execution tools. In the current case study, the tool selection and evaluation will focus on execution tools. The preparation of the application elements primarily involves the 
generation of machine models and a relatively simple context model. These can be created using tools already available within the company, as seen during the application validation activities.

\subsubsection{Tool selection}

In order to select execution tools that provide the required functionality, the researcher broke down the Virtual Annotation application to its core functions.

1. Generate - Prior to the review and annotation session, a virtual model of the topic of interest needs to be generated. As the application aims to support early stages of the PDP, the model generation will generally involve low-fidelity models based on initial specifications or concept sketches, as seen in the application validation.

2. Review - The model(s) generated for the session should be visualised in an appropriate virtual context. Furthermore, session participants and/or the session moderator need to be able to navigate through this environment (e.g. walk and/or look around). The review may also require modifications to be made to the model, the layout of the scene or the context itself.

3. Annotate - The annotations made during the review session need to be captured and stored in such a way that they can easily be retrieved and structured afterwards. The structuring of the annotations can be based on annotation properties such as category, priority, timestamp, or author.

Having established the required functionality, the researcher conducted a desk research to identify three directions of software tools that provide these functions.

1. Custom Software - The functional prototype that was developed for the validation of the application relies on standard technologies to provide the required functionality, such as WebGL for 3D rendering and a MySQL database for storing and structuring annotations. The development of a custom solution for the Virtual Annotation application by an external partner is therefore considered feasible.

2. CAD Extensions - The company's existing CAD system can be extended with plugins that provide the desired functionality. CAD systems already provide some of the main functions, such as the generation of models and to a certain extent the review of models. The annotation and documentation functions can be added using specific plugins (e.g. eDrawings $^{2}$ ) or by combining the CAD system with external applications or devices that provide this functionality (e.g. CADFaster $\left.{ }^{3}\right)$.

3. Integrated Suite - Software suites such as $3 D V I A^{4}, C A T I A^{5}$ and $N X^{6}$ provide integrated solutions for modelling, interactive visualisations, group collaboration and data management. Although these suites provide the required functionality, it requires a significant investment in the suite itself, and, given its extensive range of sub applications and functionalities, it is expected to require additional training to realise applications like the Virtual Annotation application.

\footnotetext{
${ }^{2} \mathrm{~A}$ mobile application that displays 3D models, see http://www.edrawingsviewer.com

${ }^{3} A$ collaborative 3D model sharing tool, see http://www.cadfaster.com

${ }^{4} \mathrm{~A}$ commercial 3D development platform by Dassault Systemès, see http://www.3dvia.com

${ }^{5}$ Professional CAD system by Dassault Systemès, see http://www.3ds.com/products-services/catia/

${ }^{6}$ Professional PLM system by Siemens, see www.plm.automation.siemens.com/en_us/products/nx/
} 
The above tool directions were discussed with the designers involved in the case study. The development of custom software (either outsourced or by the company itself) was not considered a feasible option. Firstly, the company is not interested in nor capable of developing software. Secondly, while it would result in an application that is tuned to the company's requirements, it would only support the Virtual Annotation application.

The other two options were both considered feasible. However, designers indicated that a drawback of extending the current CAD system with plugins would again result in a very task specific solution. Therefore the option of investing in a more extensive software suite was also considered. Although the designers acknowledged the risk of the software being complex, the advantage of a providing a broader set of possible applications in the long run is considered at least as important. Taking this long term vision into account, it was decided to focus the remainder of the case study on the Integrated Suite direction.

\subsubsection{Tool evaluation}

Following the conclusion of the tool selection phase, the final step in the case study is to evaluate the selected tool, namely the Integrated Suite option that was explained in the previous section. Discussions with the participating practitioners lead to the conclusion that $3 D V I A^{7}$ was the most feasible option for this company. There were two main reasons that supported this decision.

- The manufacturer of the integrated suite also provides the company's current CAD suite. This allows for a smooth interaction between the company's CAD files and the new suite as no file format conversions are required.

- The company was already aware of the integrated suite, as they also considered using it for automatically generating technical documentation from CAD files.

The 3DVIA suite contains various specific applications, including Composer (facilitates generation of documentation based on 3D models), Player (a web-plugin for sharing 3D models online), Shape (a low-end 3D modelling tool), and Studio (a game engine). 3DVIA Studio can be used for the realisation of the Virtual Annotation application. This tool supports the creation of interactive 3D environments and has interfaces to various software and hardware resources (e.g. remote input devices as also used in the application prototype). The evaluation of 3DVIA Studio is based on a desk research conducted by the researcher. It was not feasible to organise a hands-on workshop or test session with the actual product, mainly because 3DVIA is a commercial product. The researcher therefore reviewed instruction and documentation material in order to establish the feasibility of using this tool to realise the desired application.

\section{Evaluation results}

The desk research investigated two aspects of realisation, namely the preparation of the application and the execution of the application.

The preparation primarily involves the creation of a virtual context in which the annotation sessions take place. The context should resemble an average factory in which the food processing machines operate. As mentioned in the previous subsection, importing the company's CAD models into this environment is straightforward. However, during the test sessions it was found that it is recommended to use stripped versions of the 3D models, only containing 'visible' parts. Context elements, such as generic factory machines, storage containers, doors, fork lifts

\footnotetext{
${ }^{7} \mathrm{~A}$ commercial 3D development platform by Dassault Systemès, see http://www.3dvia.com
} 


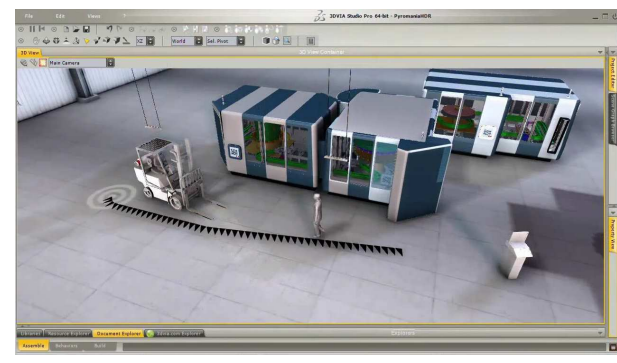

a. 3DVIA Studio provides a straightforward visual interface for preparing virtual environments.

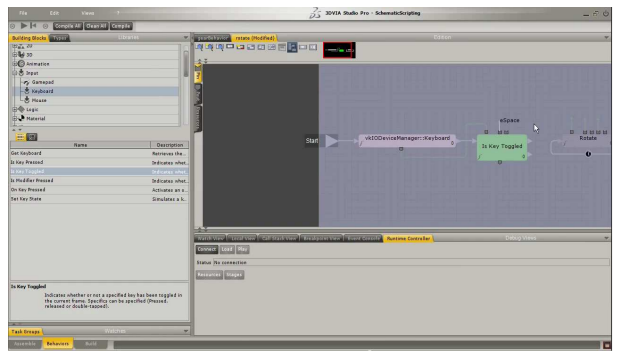

b. 3DVIA Studio supports the use of schematic (visual) programming as well as regular $\mathrm{C}++$ code.

Figure 6.9 3DVIA Studio.

and user avatars can be obtained from either online model repositories, or 3DVIA's own model repository. The application allows users to drag-and-drop objects from these repositories into the virtual environment (see figure 6.9a). Overall, given their experience with CAD software, the preparation of content is not expected to be an issue for the participating practitioners.

The execution of the application is considered more challenging. One of the core functions of the Virtual Annotation application is that users can add written or sketched annotations to specific locations in the $3 D$ environment, and structure these annotations in a database. This functionality is not directly available within 3DVIA Studio. While the tool does provide an extensive programming environment (see figure 6.9b) and supports communication with external applications such as a database, it will require custom development to realise this functionality. Although the development of custom functionality in 3DVIA Studio only has to be done once, it should still be taken into account for the realisation of the Virtual Annotation application.

Overall, the selected tool is considered to be an appropriate means for the realisation of the Virtual Annotation application. However, it is argued that the required development of specific functionalities would not have been worth the investment in such a suite if the company did not intend to pursue other VR applications as well; based on the experiences the company gained during the case study and the research in general additional opportunities were identified that can also be realised using this suite (including e.g. applications of augmented reality for marketing and sales purposes).

\subsection{Conclusion}

The Virtual Annotation application provides a virtual environment that facilitates the presentation, review and annotation of early stage product concept representations. The application facilitates discussions that lead to a shared and thorough definition (or refinement) of product requirements and specifications. While the application primarily facilitates internal communication rather than communication between designers and end-users, it was indicated by partcipants that external stakeholders, such as customers or end-users, could also be included in Virtual Annotation sessions.

With respect to the companies involved, the case study shows similarities with case study 2 , in which a truck manufacturer was involved. Some of the experiences gained in case study 2 affected how the researcher approached case study 3. For instance, a more concrete description 
of the desired application was defined to prevent the focus to shift too much during the remainder of the case study. Furthermore, the role of the application prototype was different in the sense that it only provided the role of validating the application, rather than serving as a starting point for an actual tool (as happened in case study 2). These aspects (and other reflections regarding the methods used in the case study) are further discussed in chapter 8.

Although applications similar to the Virtual Annotation application have been published before, they had not yet been applied in early stage design meetings. It was shown that, with minimum preparation by designers, a virtual environment can be realised that facilitates

- Effective and efficient introduction of a product concept to stakeholders not yet involved in the project

- Identification and validation of initial requirements regarding various aspects, including e.g. use, installation or maintenance

- Discovery of new features and functionality for an existing product concept

- Structured documentation of all the feedback, comments and annotations gathered during a review session

It was found that the functionality required to execute this application is already available in various classes of software, ranging from stand-alone applications to extensive CAD suites. The current company's intention to also explore other VR applications led to the decision to focus on these CAD suites for the realisation of the final Virtual Annotation application. 



\section{$7 \quad$ Generalisation}

In this chapter, generalised insights are derived from the three company specific case studies regarding the application and realisation of $V R$ in the early stages of a user centred product development process. The generalisation method introduced in chapter 3 was used to conduct three cross company evaluation sessions, in which the results of a particular case study are translated to the design contexts of the other two companies involved in the research. These translations indicate whether the applications defined within a specific case study can also be used in other design domains. Subsequently, it is investigated whether the realisation of these applications differ between design domains. Together, these insights will be used to propose a generic approach for practitioners to get started with VR in a UCD process.

\subsection{Cross company evaluations}

The CCE approach that was introduced in section 3.3 has been used to structure the three CCE sessions. Each session covered the following steps.

1. Case study review - The results of the case study are presented to the participants. The presentation outlines the main events of the case study, such as workshops, demonstrations and evaluations. The presentation is co-hosted by company representatives who give feedback on how they experienced the case study and how the results affected their work.

2. Demonstration of results - The application and tool selection resulting from the case study are presented to the participating companies. If possible (depending on the state of the application prototype and the availability of supporting tools), participants are invited to engage in a use case and experience the application first-hand.

3. Discussion - In groups the representatives of the participating companies are given the assignment to translate the presented results into something useful to their company. This requires them to describe how the application would be used (e.g. purpose) and who would be developing and/or using it.

4. Evaluation forms - After the session the participating practitioners fill out an evaluation form. The CCE evaluation forms are included in the appendices of the respective case studies. The results of these evaluations are included in the appendices of the individual case studies, and integrated as references to supporting questions and quotes in the following discussion of the CCE results. 


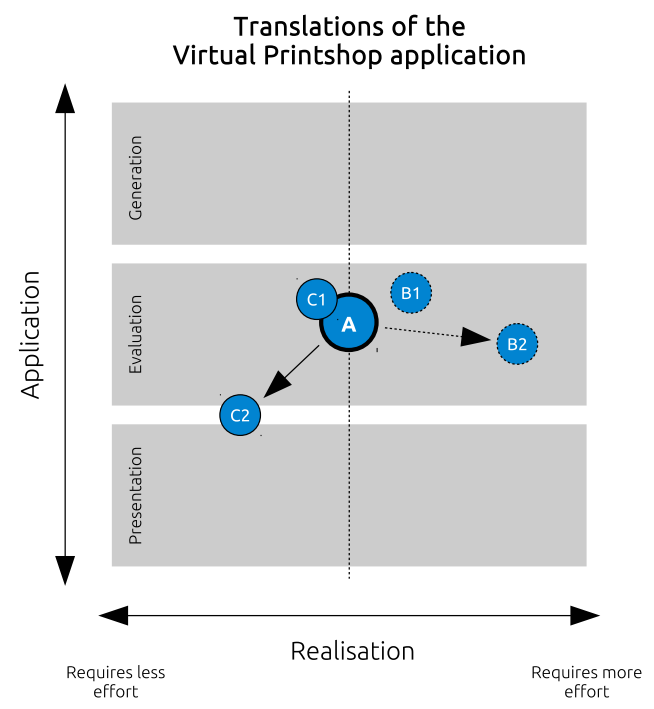

Figure 7.1 Results of CCE 1. Company B made two translations; B1 represents the 'cabin as context' application, which was not considered very useful for the company. B2 represents a driving simulator, which is a relevant application but no longer considered a translation of the Virtual Printshop application. Company $\mathrm{C}$ made a direct translation of the application $(\mathrm{C} 1)$, but also proposed a simplified version (C2) that would support presentation activities rather than evaluation activities.

Using this approach, the Virtual Printshop, the Virtual Personas and the Virtual Annotation applications have been translated to applications for the design domains of the companies involved in the case studies. The next subsections briefly outline the proceedings and results of the CCE sessions.

\subsubsection{CCE 1: The Virtual Printshop}

The first cross company evaluation session started with a presentation of the case study that was carried out for company A. Two designers who were involved in the case study shared their experience with the VR application and in particular the realisation of the application. After the case study overview, the other companies ( $B$ and $C$ ) were given a demonstration of the VR application, after which they were asked how they would deploy this application in their PDP, and which bottlenecks they expect with respect to the realisation of the application.

Company $\mathrm{C}$ made two translations of the Virtual Printshop application. The first translation ( $\mathrm{C} 1$ in figure 7.1) is made by translating the content of the Virtual Printshop into content relevant for company $C$. In this case, they envisioned a virtual factory hall in which they could do $3 \mathrm{D}$ walkthroughs with machine operators or machine buyers. In their second translation ( $C 2$ in figure 7.1), the company suggests to leave out the behaviour modelling tool, and use non-interactive models for sales and marketing purposes. Here 'non-interactive models' refers to machines in the virtual environment that demonstrate behaviour, but do not respond to user input interactively. The suggestion of leaving out the behaviour modelling tool altogether was confirmed in part 1 of the post-session evaluation form (see appendix A.3.1). Question 1.2 
('are the tools presented in the case study suitable for you?') was answered with "won't use it".

Company B had difficulties with translating the Virtual Printshop application; the company participants indicated that their 'use context' is much more complex, as it usually involves roads and traffic scenarios. The remarks added to questions $2.7,2.8$ and 2.9 of the postsession evaluation form (see appendix A.3.2) support this. For instance: "Our virtual objects are more complex, especially the behaviour of other road users". After some discussion the company came up with an alternative application (B1 in figure 7.1) that uses the truck cabin as a context; within this context the driver could experience or evaluate e.g. new storage concepts or new dashboard layouts. However, the company did not consider this application to be very relevant for them. In the final presentation their application had been translated into a driving simulator (B2 in figure 7.1). Although the terminology of the reference application was used, this application was considered a different application altogether rather than a translation.

After presenting the application translations, a discussion about the realisation of the application was held. The preparational tools presented during the first part of the session (the tools from case study 1; SweetHome3D, Blender and external model repositories) were not expected to be a bottleneck in realising the application for either company. The external model repositories were received well. Interestingly, companies $B$ and $C$ indicated that they already have a set of light weight versions of their product models, currently used for making quick renders or for sending models to clients. These types of models can also be used in the virtual environments. However, as with company A, behaviour modelling (involving the definition of behaviour through programming) is expected to be challenging. Company $A$ has dedicated resources for this in the form of the prototyping department. The solution to this problem proposed by company $C$, namely to leave out behaviour modelling of the tool chain, was also considered a feasible option. Company B indicated that they already have the tools for defining behaviour (e.g MatLab), which they would like to connect to such virtual environments.

\subsubsection{CCE 2: Virtual Personas}

In the second cross company evaluation session the Virtual Persona application was presented and translated to the other two design domains. In this session, the presentation included not only an overview of the case study conducted for company $B$, but also a fictional test case in which the other participating companies ( $A$ and $C$ ) could experience the Virtual Persona application. After the test case the companies were asked to describe how they would deploy the Virtual Persona application in their PDP, and whether or not bottlenecks with respect to realisation were expected.

The Virtual Persona application was generally well received by the other companies. Regardless of whether companies already had experience with regular personas, they were able to translate the application to their own design context.

Company $A$ was already familiar with using personas in its PDP, and was able to make a direct translation of the original application ( $A$ in figure 7.2 ). The familiarity with personas allowed them to identify the potential added value of using virtual personas instead of regular personas. The company participants indicated that the explicit and constant confrontation with the persona and the use context helped with focusing a design session. In the post-session evaluation form (see question 1.5 in appendix B.3.1) the company referred to this as "alignment; everyone is forced to look at the situation/context from the same perspective". It also helps place an existing concept in a particular use situation (e.g. for evaluation) and stimulates and triggers the generation of new ideas. Furthermore, it was found that the fidelity of the personas (e.g. the way they walk around and move their body) was sufficient for these applications. The 


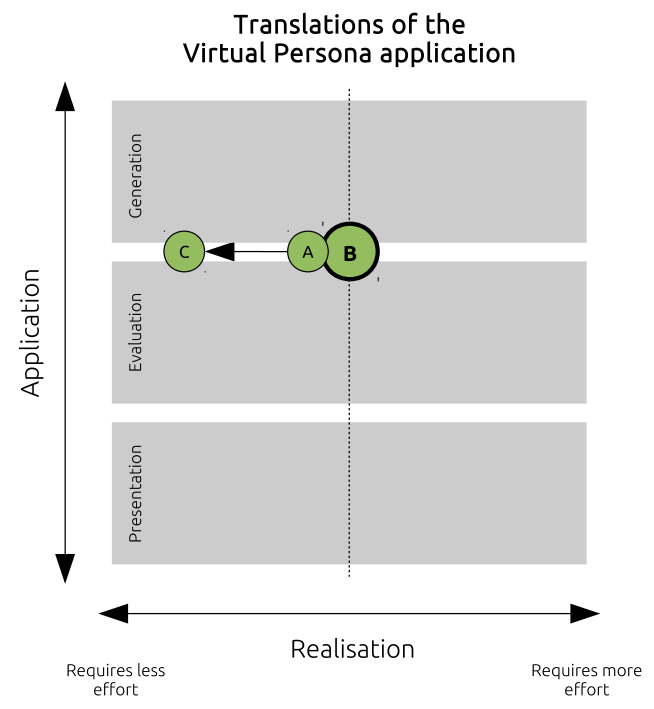

Figure 7.2 Results of CCE 2. Company A, familiar with personas, made a direct translation of the original application. Company $\mathrm{C}$ proposed the same application but indicated that a lower fidelity of the virtual avatars would be sufficient.

company's prototyping department did not see major bottlenecks with respect to the realisation of such applications. In the post-session evaluation form the prototyper who participated in the session named tools that could be used for this purpose.

Company $\mathrm{C}$, which was unfamiliar with personas, indicated that they would initially define personas based on functions (e.g. operator, maintenance engineer, installer, etc.) rather than personal characteristics. The company's designers indicated that this type of persona does not need a high level of detail; it is sufficient to recognise which function is represented, but the persona does not need to move or act as realistically as seen in the application prototype. This was confirmed by question 2.11. For company $C$ the primary purpose of the application ( $C$ in figure 7.2) would be similar to the original Virtual Persona application, namely to review product concepts from the perspective of specific types of end-users. In the post-session evaluation form the company stated the anticipated benefits as "the application enables a confrontation with different perspectives on our product, leading to more founded decisions and the discovery of 'hidden problems' in early stages of the design process" (question 1.5).

The realisation of this application would primarily rely on CAD models made by the company's engineering department. As the company does not require complex avatar models, they did not expect bottlenecks in this area; simple static avatars (with specific shapes or textures to represent a particular type of user) would be sufficient.

\subsubsection{CCE 3: Virtual Annotation}

The third cross company evaluation featured the Virtual Annotation application that was developed for company $C$. The session involved a presentation outlining the proceedings of the case study, as well as a test case in which designers from companies $A$ and $B$ experienced the 


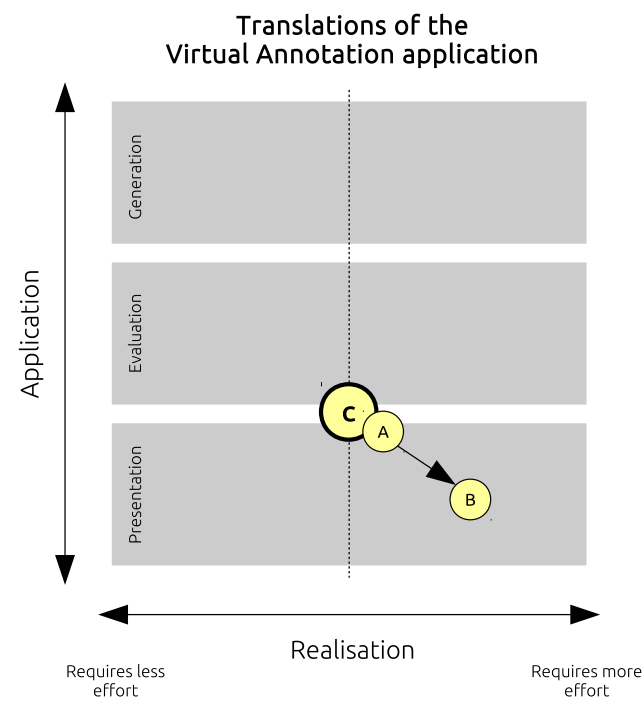

Figure 7.3 Results of CCE 3. Company A made a direct translation of the application. Company B indicated a need for more detailed models, and considered the application to primarily support presentation activities rather than evaluation or generation activities.

Virtual Annotation application first hand.

The companies involved in CCE3 indicated that the Virtual Annotation can be used in various phases of the development process and could support various early stage design activities including idea generation and concept evaluation. The companies agreed on the added value of making annotations and storing these in a structure for post-session access. This was supported by relatively high scores these functions received in answer to question 2.2 of the post-session evaluation form of CCE3 (see appendix C.2.1).

Company $\mathrm{B}$ indicated that in the automotive design domain the relation between the virtual products and the virtual context is more complex and relevant than in the presented application (see questions 1.4 and 2.1 in appendix C.2.1). In automotive applications (B in figure 7.3) designers from company $B$ envision interactions between the product and the context; a truck may drive around a virtual world. Furthermore it was also indicated that "the situation is too much simplified, which prevents detailed problems from being noticed" on the post-session evaluation form (question 1.4). The lack of details in the application prototype may cause problems to be overlooked during early stage evaluations, especially when reviewing complex products or components. Consequently, the company did not consider the presented application useful (question 3.1) in its current form. The company would therefore use their current CAD suite as a basis for the annotation application. The required additional functionality (annotation management) would have to be integrated with (if not already part of) their tool suite.

Company A considered the prototype application to represent a sufficient level of fidelity for their intended application (A in figure 7.3); it can facilitate early stage design reviews and generation sessions, especially when multiple stakeholders are involved who do not have indepth knowledge about the product concept. With respect to realisation, the virtual annotation environment was compared to that of the virtual printshop, and it was concluded that neither 


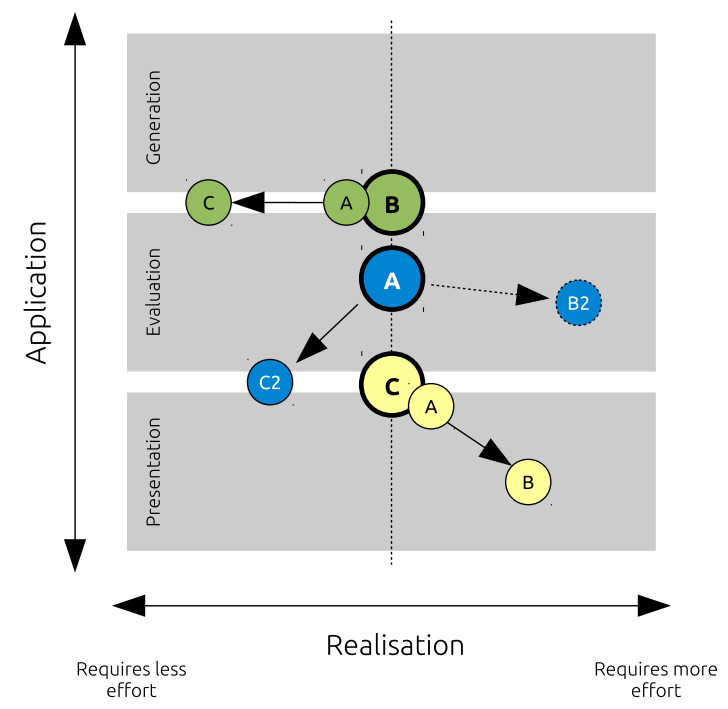

Figure 7.4 Results of CCE's.

the virtual context (which would typically be an office environment for company A) nor the products (printers, office equipment, etc.) would be problematic to create. The functionality of creating, storing and structuring annotations would preferably be added to their tool chain by using plugins or extensions for the CAD tools they are currently using, rather than creating a custom application from scratch for example, or buying in an extensive tool suite to support this functionality.

\subsection{Analysis}

The CCE sessions provide insights into how designers from the different companies realised the translations of the case study specific results into relevant VR solutions for their own design context. Figure 7.4 shows an overview of these translations, illustrating three types of translation.

1. Content - When both the VR application and the realisation of the application match the company's requirements, a direct translation can be made by modifying the content of the application (e.g. the application's virtual assets or the virtual context). This type of translation was most commonly seen in the CCE sessions; it requires designers to use preparation tools to create different content for the application, but it does not change the required execution tools.

2. Application - After experiencing the reference application during the CCE sessions, designers often fine-tuned the application of VR to their particular PDP. For example, the Virtual Printshop presented in CCE 1 was translated from a purely evaluation application to a presentation application by company C. Similarly, company B expects the Virtual Annotation application to mainly support presentations rather than evaluations. 
3. Realisation - Designers used the means of realisation to align the VR solution with their PDP. For example, when higher fidelity models were required by a company, the company would propose to use their current CAD tools to provide these models. Alternatively, in CCE 1 it was found that company $C$ did not expect to use 'behaviour modelling', and consequently decided to take these preparation tools out of the proposed tool chain.

The three types of translations affect each other. Changing the focus of a VR application can affect the required means for realisation in either direction. Changing the content of a VR application may in turn require different preparation tools, or change the focus or purpose of an application.

\subsubsection{Translation strategies}

All three companies involved in the CCE sessions had their individual approaches for making the translations. The following subsections further describe these approaches using the three types of translations introduced earlier.

\section{Company A}

Company $\mathrm{A}$, involved in the development of professional printing systems, generally followed an application driven approach; the reference applications were translated to their own domain by changing the content of the application, as shown in figure 7.4 , followed by an assessment of whether or not they had access to the tools required for the realisation of this new application. Overall, the translations of this company were quite similar to the reference applications, meaning that neither the proposed application nor the realisation required significant modifications.

There are two reasons that might explain this. Firstly, of the three companies, company A has the most experience with conducting UCD activities; they are familiar with conducting usability evaluations with end-users, with conducting contextual inquiries, with various forms of early prototyping, and with doing user interviews and surveys. This might have helped them link VR applications to specific UCD activities. With the Virtual Persona application, for example, it was found that the company is already familiar with personas, which helps with understanding the concept of personas, but also with understanding the shortcomings of traditional personas and the added value that Virtual Personas could bring. A second important aspect is that the design department involved in the research includes a dedicated prototyping team that is in charge of creating digital interactive prototypes. The tools and skills used by this department overlap with the tools and skills needed to prepare or execute certain VR applications. Consequently, during the CCE sessions the company's discussion about realisation often ended with the participants saying that their company's prototyping department would take care of it.

\section{Company B}

Company $\mathrm{B}$, involved in the development of trucks, generally followed a content driven approach. While the applications were generally found to be useful for the company, the fidelity of the application's content, and consequently the means needed to realise this content was generally considered too low for the company's needs.

Several factors affected this position. Firstly, the company itself mentioned that the complexity of their product is so high that it is impossible to fully represent it in simplified early stage models. This was also found in the results of the post-session evaluation forms; the participants generally indicated that the presented level of detail (e.g. in the applications of 
CCE1 and CCE3) were considered too low for their design domain. Secondly, the company strives to maintain a streamlined tool chain throughout their development process. This means that early stage UCD applications would preferably be integrated with this tool chain, resulting in a lot of constraints and requirements for the realisation of the VR application (e.g. model formats, model reusability, confidentiality). Thirdly, it was found that the company primarily involves end-users or end-user representatives to test and validate product proposals, rather than to generate or evaluate new product concepts. Testing and validating complex products, such as in vehicle information systems, involves relatively detailed functional models. As a result of these factors, the company made most of the VR application translations by increasing the fidelity of the content, in line with the content they already have (CAD models, Matlab models, etc.).

\section{Company C}

Company $\mathrm{C}$, involved in the development of industrial food processing solutions, used an approach similar to company A. This company mostly used an application driven approach; the company tried to translate the application's content to result in a useful application, and then assessed the feasibility of the means needed for realisation. Although this approach is similar to that of company $A$, the focus of company $C$ was clearly different, which resulted in different translations of VR applications as well. Company $\mathrm{C}$ generally showed a slightly reserved attitude with respect to e.g. the fidelity of the application's content, and consequently demanded less extensive means for realisation.

There are several possible reasons for this. Firstly, the company is relatively unfamiliar with the UCD activities that are facilitated by the applications. As a result, it can be expected that the company does not immediately invest in an extensive tool chain to realise the new application. Secondly, unlike company A, company $C$ does not have a 'fall-back' department for the realisation of the VR applications. Consequently, they have to assess carefully how and by whom the application is going to be realised. However, rather than solving this issue by trying to extend their current tool chain forwards in the PDP (as seen with company B), the company has been looking at alternative solutions that provide dedicated means for the realisation of various VR applications.

The fact that company $C$ was the last company involved in the research project may have contributed to this strategy; prior to their own case study, they have seen and experienced the other VR applications, and may have become aware of a larger set of potentially interesting applications.

\subsubsection{Interpretation of results}

While each company has its individual translation strategy, based on e.g. an existing tool chain, company culture or the attitude towards user involvement, there are interesting similarities in the ways the companies conducted the translations during the CCE sessions.

The overview of translations illustrated in figure 7.4 shows that most of the translations involve direct or horizontal translations. This indicates that the participating companies primarily used the means for realisation (i.e. preparation of content and execution tools) to translate the proposed VR solutions. The VR solutions that result from this translation process address a company specific challenge by providing specific content or behaviour. However, it is found that the core of the application remains the same, regardless of how it is realised. The results of the post-session evaluation forms support this finding. When asked about the purpose of the 
presented application, the participants generally agreed, although the primary and secondary purposes were not always consistent between companies.

In other words, given a concrete 'reference VR application', the companies were able to identify the appropriate means for realisation within their domain. This identification process is company specific, as shown by the three different translation strategies, and is affected by the following factors:

1. The company's present tools/tool chain - an existing tool chain can provide constraints within which new tools or functionalities need to be realised. In case study 2, for instance, the company eventually preferred to integrate the Virtual Persona functionality with their existing CAD and PLM software.

2. The company's present skills (e.g. in modelling or programming) - present skills in modelling or programming can be used for the preparation and/or execution of VR applications. Sharing these resources saves time and money in training or in the acquisition of new tools.

3. The company's attitude towards user involvement - end-users or external stakeholders in general can be involved directly or indirectly. Sometimes a company's internal confidentiality policies prevent direct involvement. This requires designers to realise applications that support indirect involvement, as seen in case study 2 .

4. The company's budgets (e.g. time or financial) - Budgets may prevent designers from investing in tool specific training or new software, limiting the possibilities for realising the desired application.

The priorities that companies assign to these factors determine the overall translation strategy. When an existing tool chain needs to be used as a basis for realising VR applications, the translation strategy will primarily be tool driven, as for instance seen with company B. As such, the process of determining an appropriate direction for realisation is very much company specific.

\subsubsection{Generalised model}

Having shown how three applications that were originally developed for specific design domains were translated to other design domains, it is concluded that these applications share a common core that addresses needs or opportunities in all three design domains. Therefore, a generic description of VR applications that facilitate UCD activities can be composed by identifying the similarities between the three core applications. This generic description can help with 1 ) understanding the VR application principles that contribute to UCD activities, 2) forming the basis for other VR applications that facilitate early stage UCD activities, and 3) defining generic approaches for the realisation of these applications.

Figure 7.5 graphically represents the relation between the designers, the VR applications and end-users. The model shows how the VR applications that resulted from the case studies and CCE sessions form a range of applications that provide an interface between design practitioners and end-users. The following subsections present a description of a generic VR application that forms the basis of these applications, and outline the scope in which this generic application can be used. 


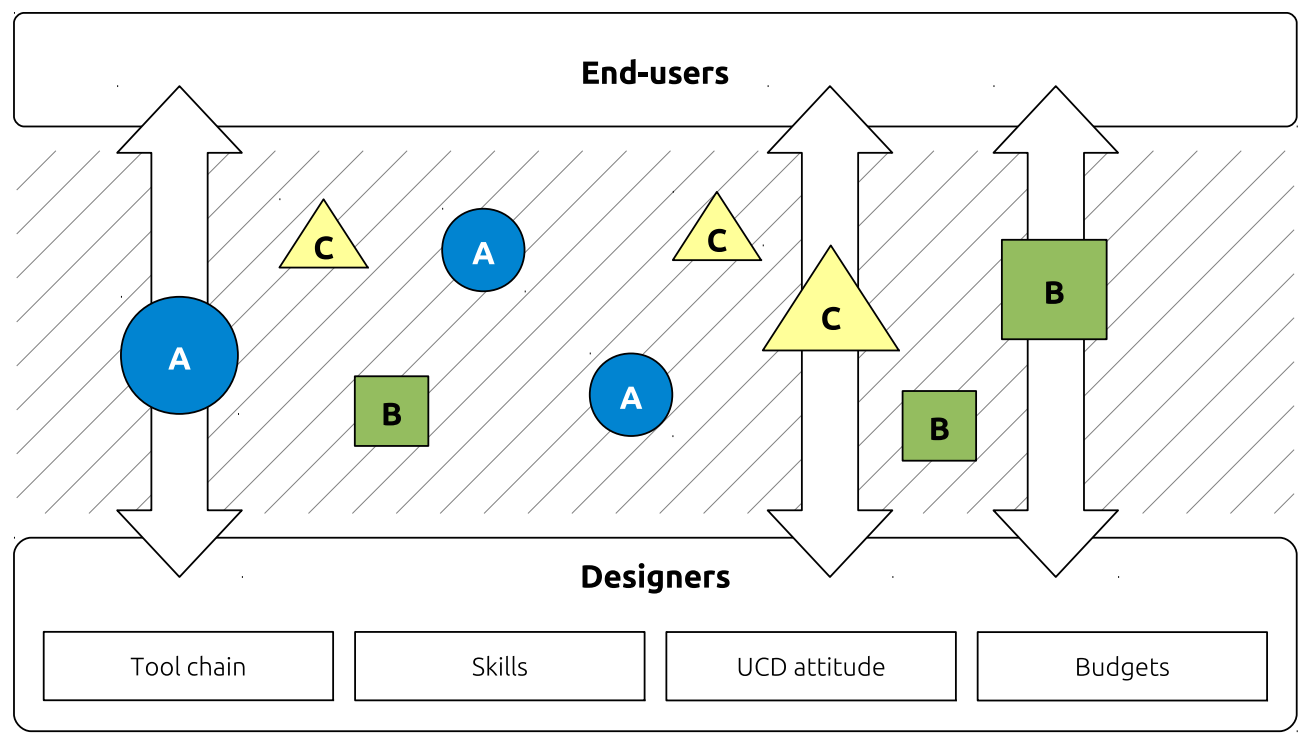

Figure 7.5 Generalised model of the relation between designers, VR applications that facilitate early stage UCD activities, and end-users. The applications form a communication channel between designers and end-users.

\section{Application core}

The common role of VR in applications presented in the case studies was to facilitate stakeholder involvement in design activities by providing an integral and interactive virtual representation of future use situations. This involves the following elements:

1. Virtual representation - The virtual representation of future use situations can be implemented through various techniques, as shown during the case studies; it ranges from augmented reality environments to fully virtual 3D walkthrough scenes.

2. Future use situation - The future use situation describes a use context, a product, a representation of an end-user and the interactions that take place between these elements.

3. Stakeholders - Although the research focused on end-user involvement, the case studies have shown that a larger group of internal or external stakeholders can be involved, depending on the purpose of the design activity.

4. Design activity - The design activities contribute to achieving a certain design task, such as the presentation of a concept, the generation of new concepts, or the evaluation of product concepts.

The virtual representation (see figure 7.6) facilitates communication between stakeholders by providing an explicit and complete representation of the future use situation. This representation enables uninformed stakeholders such as end-users to quickly understand product concepts, and to interpret the product's interactions with a use context or the end-user himself. 


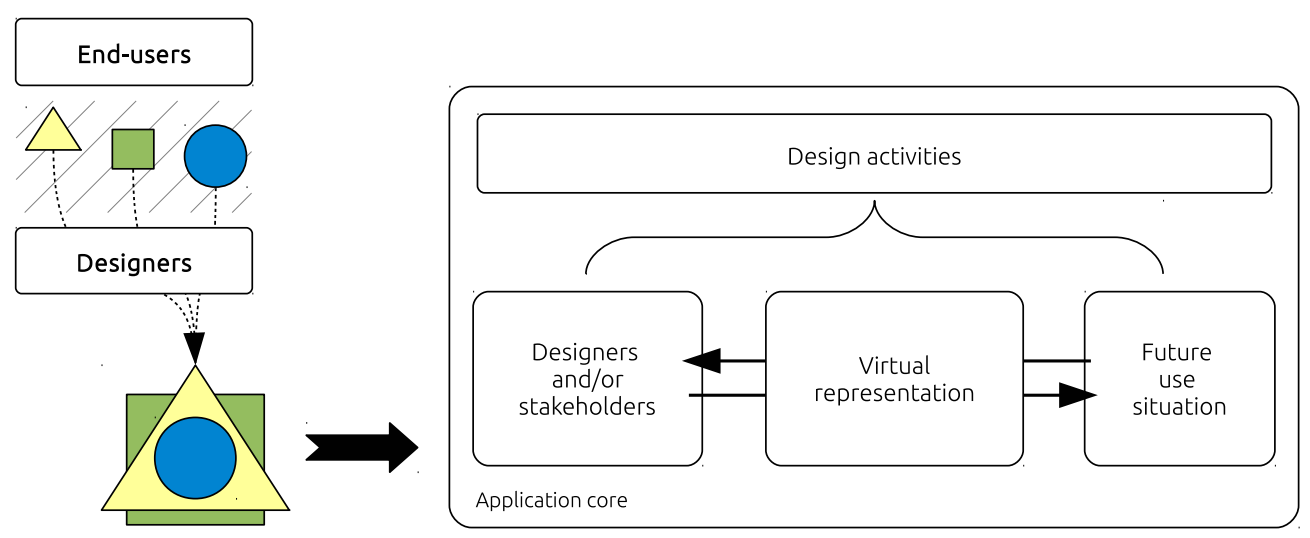

Figure 7.6 The common elements of the VR application core. The virtual representation of a future use situation facilitates various design activities involving designers and/or stakeholders.

As such the virtual representation forms a bi-directional communication channel; end-users can ask designers for clarifications regarding for example the behaviour of a virtual product concept, while designers can ask end-users for feedback on the usability or perceived benefits or drawbacks of a product concept. Even when end-users are not directly involved, the virtual presence of an end-user in the virtual representation can help designers think from the perspective of the end-user, as for instance seen in the Virtual Persona application.

Both the case study specific applications and the generalised applications use relatively low fidelity representations of the use situations. There were some exceptions to this general rule. For instance, when the users of a virtual environment (for instance end-users working in the Virtual Printshop) need to recognise a real-life situation, the virtual environment should provide sufficient references to it. Furthermore, there is a difference between the fidelity of the context and the fidelity of the products inside the virtual environment; the context generally acts as a wallpaper that should be recognisable, but not fully realistic or high fidelity. Product models on the other hand should make use of available design information, which is related to the stage in the PDP in which the application is deployed.

\section{Application scope}

The primary role for $V R$ in the application core is to provide a concrete and integral representation of future use situations without the need for (or availability of) detailed design information. The case study applications have demonstrated how low-end techniques provide sufficient means to support this role. However, this role is only valid (or relevant) in a specific part of the PDP.

The very early stages of the PDP are characterised by a lack of detailed design information and high flexibility (the space for design changes and modifications). In these stages virtual representations of future use situations can contribute to the PDP, as illustrated in figure 7.7a. They help designers with eliciting new design information from (or about) end-users or other stakeholders, using the core elements discussed in the previous section.

However, as the product concept becomes more and more concrete, it also becomes less challenging to imagine future use situations; when physical prototypes become available, actual field tests could be carried out with end-users. While VR could still play a role in these stages, 


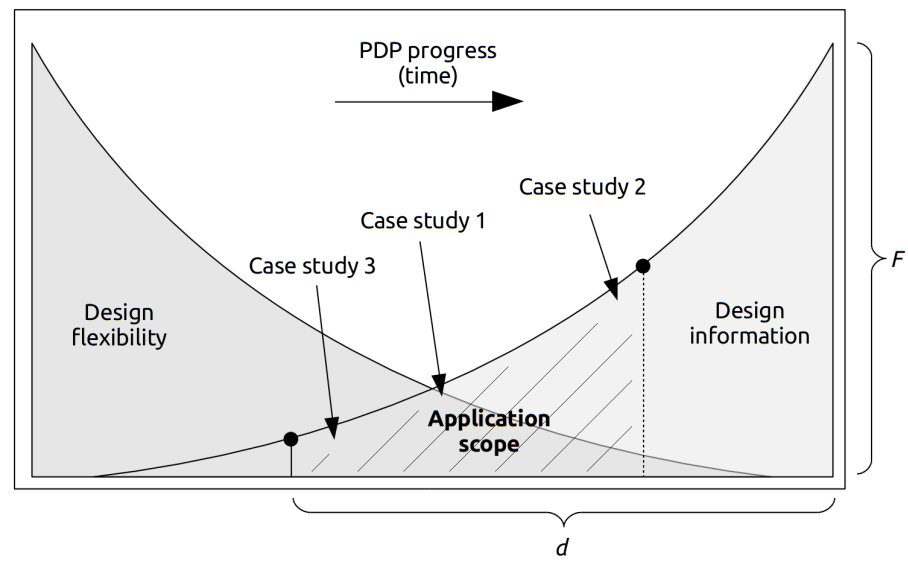

a. In the early stages of the PDP (distance $d$ to the final product $F$ is large) virtual product and use context representations allow end-users and designers to 'look into the future'.

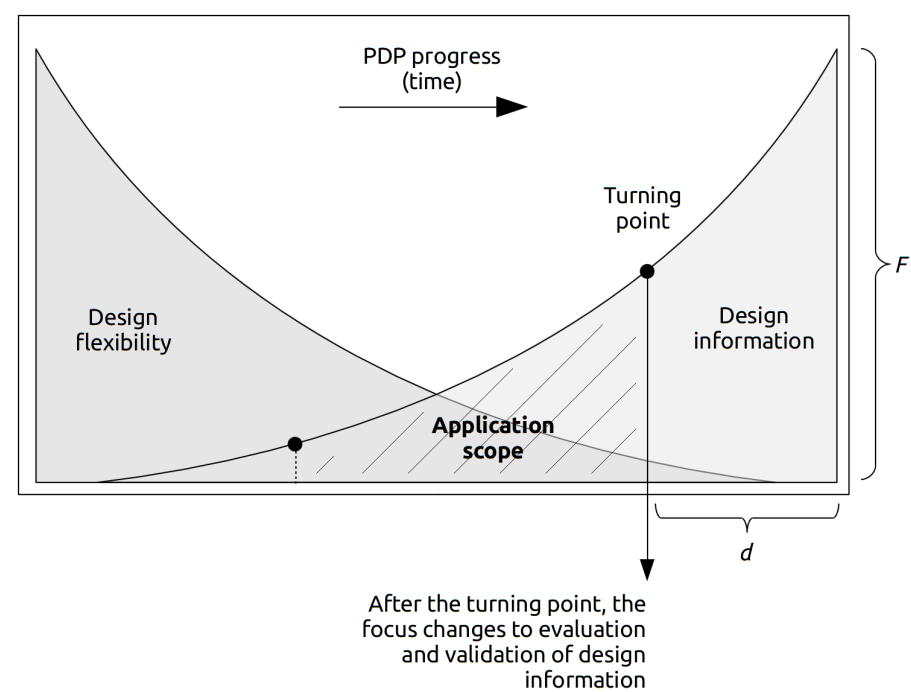

b. As the PDP progresses, after a certain turning point the added value of using virtual product and use context representations decreases as more accurate means become available (e.g. detailed CAD models or physical prototypes).

Figure 7.7 The scope in which the generalised application is considered valid. 


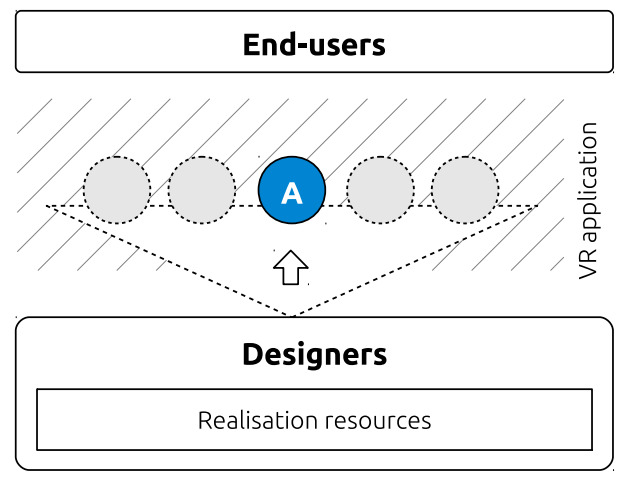

a. The application definition step involves the exploration of possible VR applications within a certain design context.

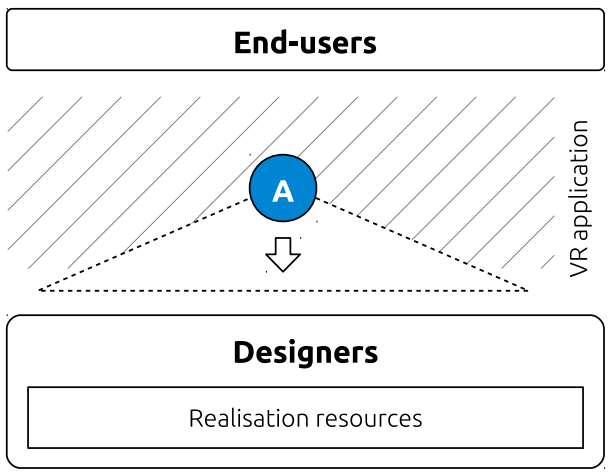

b. The tool selection phase identifies appropriate preparation and execution tools.

Figure 7.8 The realisation for VR applications involves an application definition phase and a tool selection phase.

the focus of these activities is fundamentally different from those in the early stages; rather than using VR to gather new information from stakeholders, VR (or another supporting technique) is used to present existing design information to stakeholders in order for them to evaluate or validate the information.

Although it is difficult to pinpoint where the early stages end and where the next stage starts, it is argued that case study 2 provides a good indication of it. Especially compared to case studies 1 and 3 , case study 2 was of a reflective nature. While case studies 1 and 3 used as little means and low fidelity models as possible to anticipate end-user behaviour or requirements, in case study 2 the designers focused on evaluating and validating fairly detailed product concepts (i.e. concepts based on detailed design information, such as MatLab or CAD models). This reflective perspective is illustrated in figure $7.7 \mathrm{~b}$.

\subsection{Approach for realisation}

Having established the description of a generic VR application that facilitates early stage UCD activities, this section further discusses the realisation of such an application. As explained in chapters 1 and 2 , it was originally expected that the main bottleneck for the adoption of VR in the early stages of the PDP was a lack of 'designer friendly' tools to realise VR applications. The results of the CCE sessions however show that there are effective applications of VR that can be realised using present tool chains or off the shelf hardware and software.

This is partly due to the fact that off the shelf hardware and software are mature technologies and typically include proper documentation and professional support. Furthermore, during the case studies practitioners proved to be able to identify means from within or outside their present tool chain that could support the realisation. This indicates that there is another factor that reduces the threshold for designers to realise the VR applications themselves. It is argued that having a clear and collaboratively established definition of the envisioned VR application helps with reducing the threshold for design practitioners to start using new tools. Two explanations for this finding are suggested; 
1. The application provides a concrete reference for deciding whether or not a certain feature is needed, and consequently whether or not a preparation or execution tool should support this.

2. The application has been defined in a collaborative and multidisciplinary setting (i.e. the VR exploration workshop), which may have created commitment to the realisation.

Based on these findings it is concluded that the realisation of VR applications depends not only on usable and accessible tools, but also on a collaboratively established definition of the desired VR application, and a well-considered selection of appropriate means to realise this application. The way in which these aspects are taken into account in an approach for realisation very much depends on the company involved. As explained in section 7.2.2, companies have their own strategies with respect to the realisation of VR applications. Consequently, the following two subsections only provide an overall recommendation on how the aspects could be taken into account in practice.

\subsubsection{Application definition}

The first step is to identify applications that can be realised within a given design domain (see figure 7.8a). Depending on the company's starting point this can be a very explorative step (when the company is unfamiliar with VR and/or UCD), or a specification step (when the company already has an idea of what UCD activities are to be facilitated). When the company is unfamiliar with VR and/or UCD, the application definition can be supported by the VR exploration workshop, of which the format has been outlined in chapter 3 and which will be reflected on in chapter 8 . The workshop facilitator should be well informed about available VR techniques as well as potentially interesting UCD techniques. Alternatively, when the aim of the application definition is to further specify an already established application, a more in-depth discussion with (external) VR experts is considered more effective.

In both cases however it is considered important to collaboratively establish a description or specification of the VR application that is to be realised.

\subsubsection{Tool selection}

The second step aims to identify tools required to realise a specific application (see figure 7.8b). Depending on the available realisation resources, companies can decide to focus on reusing existing resources or invest in new tools, skills or even additional applications. Similar to the application definition step, the tool selection process can be carried out internally (i.e. within the company) or by relying on external expertise. During the case studies, the researcher fulfilled the role of an external expert. This situation follows a more traditional consulting approach, in which the expert selects appropriate execution and preparation tools based on requirements expressed by a company. This approach may be more effective and efficient when the company is unfamiliar with VR technologies in general, or when the company is interested in acquiring specific new tools.

Alternatively, when the application can be realised using off the shelf techniques or tools already available within the company, the tool selection could be carried out internally. To support (internal) tool selection, the following guidelines have been defined based on the experiences gained during the case studies. 
- Consider the current tool chain

- Companies involved in product development often already possess the tools and skills required for the preparation of $3 \mathrm{D}$ assets (e.g. CAD software).

- Modern CAD applications also provide support for executing VR applications. Sometimes the functions are built-in (e.g. an interactive walk-through function), while plugins can also help with providing specific functionality (e.g. model annotations).

- If the VR application requires integration with other tools such as simulation software (e.g. Matlab), it is recommended to focus on larger tool suites. These suites generally provide more interfaces to external tools and data formats than smaller task specific tools.

- Consider current resources

- Design and engineering departments generally use stripped-down versions of CAD models for making quick renders or to share with clients. These 'light weight' models can also be used for VR applications.

- Model repositories such as Google 3D Warehouse provide a good source of 3D assets that can be used to support the preparation of the VR applications. The repositories provide generic models such as furniture, vehicles, humans and scenery objects.

- Consider the desired scope of VR applications

- If the company only intends to realise a single VR application, task specific tools (such as BuildAR or SweetHome3D) are sufficiently capable and easy to use without extensive training.

- Tool suites such as 3DVIA, Blender or NX provide an integrated solution for the preparation and execution of the application, but require more extensive training. They do however support a wider range of VR applications than task specific tools.

It should be noted that although both the application definition and the tool selection steps generally benefit from the involvement of external expertise (i.e. a VR expert), the actual realisation and use of the final VR application does not necessarily require external support. 



\section{$8 \circ$ Reflection}

In order to properly interpret the results of the research, this chapter reflects on the overall research approach and the methods used in the case studies and the cross company evaluations. Section 8.1 discusses the overall approach, consisting of a combination of company specific case studies and a series of cross-company evaluation sessions. Sections 8.2 and 8.2.4 reflect on how the research methods were implemented in the case studies and the cross-company evaluation sessions respectively, and explain how the methods evolved during the research.

\subsection{Research approach}

Before discussing the low-level methodologies used in the case studies and the cross-company evaluations, this section reflects on the overall structure of the research, the involvement of design practitioners in the research, and the gathering of data from the research.

\subsubsection{Structure}

The overall structure of the research consists of the sequential execution of company specific case studies, followed by cross-company evaluations. The main reasons for involving the companies sequentially (as opposed to conducting the case studies in parallel) were for the researcher to be able to focus on one case study at a time, and to be able to reflect on (and improve) the practical implementation of the case study method within each company.

Alternatively, the researcher considered to involve the companies in parallel; by conducting case studies at the three companies simultaneously, practitioners could directly share results, inspire each other and the case studies could span a more extended period of time. However, a drawback of this approach was that it might hinder the exploration of relevant VR applications; not only because practitioners from different companies might influence each other's decisions, but also because the researcher might be tempted to share technologies or prototypes between the companies. Both aspects might led to a less diverse set of VR applications. Furthermore, practical aspects such as the distance between the companies and the time required for creating application prototypes lead to the decision to involve the companies sequentially, followed by cross-company evaluation sessions.

An important consequence of involving the three industrial partners sequentially is that company $B$ and in particular company $C$ learned from the experiences gained in preceding case studies. From the perspective of the companies this is not really a problem. However, from a research point of view this learning effect may have influenced the results of the cross company evaluations. As described in chapter 7, company $C$, which had seen the results of case studies 1 and 2 prior to conducting their own case study, had quite a different translation strategy than 
company $B$ and (to a lesser extent) company $A$; they prefer to invest in a versatile software suite that supports the preparation and execution of various VR applications. The fact that the company had seen the applications of case studies 1 and 2 may have contributed to this decision.

Another consequence of this approach is that companies are only actively involved during one third of the research. In the initial stages of the research, the companies who were not yet involved in a case study indicated that they did not really feel 'involved' in the project, apart from seeing mid-term results. As this might affect the attitude of companies in the research, it is considered important to manage these drawbacks of sequentially involving the industrial partners. The following solutions have been applied during the research to cope with the effects of sequentially involving company participants.

- In the early stage of the research, inform companies about the sequential approach and explain why the companies are involved one by one.

- Keep companies informed about the progress of individual case studies, for instance by using newsletters, an online blog or regular group meetings.

- In group meetings make sure that all company representatives are actively involved, for instance by letting them share experiences or present the results of case studies to each other.

Although it is difficult to argue that the sequential approach provides a more efficient research structure than a parallel approach, it was found that the repetitive execution of the approach contributed to the definition of an efficient and effective method for conducting such case studies. Furthermore, it was found that the companies appreciate the fact that it is easy for them to schedule the case study activities, as they know the duration of the case study and the type of activities that take place. Several improvements have been made to the case study setup and the cross-company evaluation methods, as further discussed in sections 8.2 and 8.2.4.

\subsubsection{Designer centred research}

While the research investigates the facilitation of UCD activities in design practice, the research approach itself is user centred as well. Design practitioners involved in the case studies and the cross-company evaluation sessions are considered to be the users of the anticipated VR tools and applications; they will use the technologies to actually conduct the UCD activities in practice.

The active involvement of design practitioners provided the researcher with valuable insights into designers' requirements, expectations and opinions regarding VR applications and allowed for a reliable assessment of the feasibility of realising these applications in practice. As such, the approach achieved the goal of being 'application driven' as explained in section 2.4.2. Although the involvement of end-users in the evaluation of VR applications has already been demonstrated, the current research shows that design practitioners, even without extensive preparation, are also able to contribute to the definition of effective VR applications. Especially when the aim is to develop applications that can be deployed without continuous external support (as was the case in this research), it is recommended to actively involve the anticipated end-users of the application in its development. 


\section{Effects of diversity in participating practitioners}

The design practitioners involved in the case studies and cross-company evaluation sessions originate from various industrial and professional backgrounds. The industrial backgrounds range from mechanical engineering and automotive design to mechatronics design. The professional backgrounds of the participants range from industrial designers, visual designers and interaction designers to mechanical engineers and technical sales engineers. The diversity of both the companies and the participants involved in the research was primarily beneficial as it allowed for a multi-perspective view on the topics throughout the research. However, it also made it difficult to establish a common definition of 'user involvement'; there are as many interpretations of this concept as there are disciplines or industrial domains. Some practitioners consider the involvement of end-user representatives sufficient, while others mostly rely on visiting customers or on actively involving them in the design process. It was found that the perception and adoption of user involvement is strongly related to prior experience with user involvement, the organisational structure of the individual design departments, and the desired role of user centred design within the company.

The participating department of company $\mathrm{A}$ is a dedicated design department that receives input from marketing departments, but which also conducts its own user research (e.g. contextual inquiries or field tests). As such, there is a close relation to end-users, leading to primarily first-hand information about end-users. Furthermore, as a dedicated design department the designers have experience with quite a wide range of user involvement methods, which may have contributed to the company's exploring attitude in the research; they are not only used to actively involving end-users, but also to experimenting with new design methods.

The involved department from company $B$ is in charge of defining and validating specifications of specific product components, based on market insights from a marketing department and test results from a testing department. This means that the practitioners from this department have relatively few (or no) direct relations to end-users or end-user representatives. Consequently, when discussing user involvement with these practitioners, they often indicated that the marketing or testing departments were responsible for doing interviews, questionnaires or field tests. Compared to company A, the practitioners from company B were somewhat less exploration oriented, and more interested in applications that support concept validation activities, with or without end-users.

Most of the participating practitioners from company $C$ were working in the R\&D department, situated between the company's sales and engineering departments. Similar to company $B$, the practitioners had limited experience with actively involving end-users, as most of the contact with customers and end-users takes place during sales activities and is transferred to $R \& D$ and engineering by means of reports and presentations. While the position of the practitioners is similar to that of company $B$, an important difference between case study 3 and case study 2 is that in case study 3 the Virtual Annotation application crossed the boundaries of the department that was involved in the research, while the Virtual Persona application primarily supported the activities of the department directly involved in the case study. One of the reasons for this might be the fact that company $\mathrm{C}$ is smaller, which makes it easier to conduct research activities across departments. Another possible reason is the diversity of participants in the initial exploration workshop, a point which will be discussed in more detail in section 8.2.2. 


\section{Implications for this research}

From a design practice point of view, it can be argued that there is no right or wrong way of being involved in a case study as long as the result is useful in practice. From a design research point of view on the other hand, it is important to be able to focus on a particular goal, such as the identification of VR applications that facilitate UCD. Due to the variations in previous experiences with UCD and organisational structures it was quite difficult to maintain this focus in the current research. In case study 3, for example, the Virtual Annotation application introduced a new technology (virtual annotation) as well as a new design activity (collaborative concept reviews). While the application was successfully deployed and positively received, it is difficult to pinpoint whether the added value lies in the use of VR or in the introduction of a new design activity. In case study 1 on the other hand, the experience with existing UCD activities made it easier to assess the added value of VR to these existing activities (e.g. improving the representation of the use context in product evaluations).

Consequently, when actively involving design practitioners in research, it is recommended to assess and take into account the diversity of the participating practitioners. In the present research, the diversity of participating practitioners led to a wide range of applications of VR that facilitate UCD, but also in less informative results regarding the specific added value of VR in UCD activities.

\subsubsection{Gathering data}

Throughout the research the researcher obtained qualitative as well as as quantitative data. As the research aims to gain understanding why and how VR can be used to facilitate UCD activities, the focus is on collecting qualitative data from design practitioners.

The primary sources of data include:

1. Interviews conducted during field studies

2. Storyboards resulting from the exploration workshops

3. Data from validation and evaluation sessions:

- Raw video and audio recordings

- Session transcripts

- Evaluation form results

The insights gathered through these activities were always verified by presenting the researcher's interpretation of results in follow-up sessions. For example, the results of the interviews were summarised in group presentations involving the respective companies, and repeated during the kick-off meetings in each case study. The researcher's interpretation of the storyboards was presented in follow-up presentations as well as the initial 'application descriptions' that were made after the workshops. Observations from the workshops, validation sessions and cross-company evaluation sessions were made during the sessions but also while reviewing video recordings of the sessions and making transcripts. In addition to sharing these insights with the participating practitioners in follow-up meetings, the observations were also verified by comparing them to the results of post-session evaluation forms.

The aim of the post-session evaluation forms was to gather quantitative data, for instance by asking participating practitioners to grade a particular VR application, or by choosing an 
application's most relevant contribution to the design process. Unfortunately, it was sometimes difficult for participants to make such decisions; even when explicitly asking to select one property (e.g. "what is the primary purpose of this application"), often two or even three answers were ticked. Sometimes the participants explained that it was too difficult to answer those questions with a single answer, because often more than one answer was applicable. Furthermore, designers indicated that they did not always appreciate or see the added value of filling out such structured forms, partly because they had already discussed most of the topics during group discussions. Another factor that may have influenced the results of these post-session evaluations is that the participants were often tired from the session itself. Nevertheless it was decided to conduct the post-session evaluations directly after the sessions, rather than running the risk of participants forgetting information or feedback.

\section{Limitations}

While the quantitative results of the post-session evaluations do not contain sufficient grounds for conclusions on their own, they do provide means for verifying and supporting the researcher's interpretations and observations made during and after the collaborative sessions. As such, the combination of gathering and analysing qualitative and quantitative data provides a good tradeoff between efficiency and reliability. Nevertheless, several challenges were encountered during the gathering, analysis and presentation of data.

1. The practitioners involved in the case studies generally preferred to provide qualitative feedback rather than quantitative feedback. As described, participants often had trouble choosing one particular answer when several options are given. Extensive discussions allow the researcher as well as the participants to further elaborate on specific issues when needed.

2. Processing qualitative data, however, is time consuming. The effort required to process discussion transcripts in the application validation session of case study 1 for instance (see appendix A.1.2), was not proportional to the insights it provided. Therefore, in case studies 2 and 3 it was decided to use evaluation forms rather than to code the entire discussion.

3. Presenting the captured and/or analysed data is sometimes difficult because of confidentiality reasons. The storyboards created in the VR exploration workshops for instance all contain quite detailed descriptions of design process and bottlenecks, and have therefore not been included in this thesis. Furthermore, in case studies 2 and 3 actual design cases were used, preventing the publication of the concrete results (e.g. new ideas or concept improvements).

In the end it is up to the design researcher to decide how to cope with these limitations. In the current research, the researcher focused on efficiency (in order to complete three case studies in the given time) and practical relevance (in order to gain and maintain commitment from the industrial partners).

\subsection{Implementation of methods}

The overall research approach presented in the previous section is supported by several low-level methods that structure the activities in the case studies and the cross company evaluations. 
This section describes how the methods that were introduced in chapter 3 were implemented during the research and how they evolved after each case study.

\subsubsection{VR exploration workshop}

Most of the changes made during the research concern the VR exploration workshop. While the method was generally found to be very effective for generating VR application proposals within a multidisciplinary group of participants, the researcher optimised several aspects of the method.

The animated storyboards that were used in the first workshop as an inspiration and starting point for the participants had been replaced by trigger cards in the second workshop. This was done because no concrete starting points were identified in the kick-off meeting with company $\mathrm{B}$, and to see if the time required for the preparation of the workshop could be reduced. The use of trigger cards was found to be an effective way of initiating discussions that eventually led to individual storyboards. In addition to being used as inspiration for storyboards, the use statistics of the trigger cards (e.g. the popularity ranking of each card) gave some insight into which topics are considered relevant by participants. An important drawback of the trigger cards however was that, compared to case study 1 , participants had more difficulty with understanding the concept of a storyboard; in spite of the researcher explaining the basics of storyboards, it was less clear what was expected from the participants in the first round. Furthermore, it was found that the final storyboards resulting from the second workshop showed less variety in VR technologies. The lack of example storyboards could have caused this; prior to the session participants had a limited awareness of potential VR applications. In the first case study this awareness was improved by showing additional applications, while in the workshop of the second case study only an introductory presentation of VR in general was given.

The experiences gained from conducting the workshops in the three case studies have been used to compose the following final format for the VR exploration workshop.

1. Introductory presentation - The introduction explains the purpose of the session, which is to generate and discuss potential applications of VR in the PDP of the company. If necessary the presentation can also be used as a brief introduction of VR techniques to the participants.

2. Presentation of example storyboards - To illustrate the concept of storyboards, animated storyboards are presented and explained to the participants. The storyboards visualise different applications of VR in the PDP of the company. The ideas for these storyboards are derived from interviews, observations or preliminary meetings. Although the preparation is time consuming it is advised to use animated storyboards as a starting point especially when participants are new to working with storyboards. When participants are familiar with creating storyboards, trigger cards provide a more time efficient alternative.

3. Individual storyboard - The example storyboards are broken down into 8 to 10 key frames; paper frames that depict key events in the storyboard. Participants are asked to use these frames to generate their own storyboards by

(a) modifying the order of the frames in one of the example storyboards,

(b) merging example storyboards,

(c) adding customised (hand drawn) frames, and 
(d) adding technology frames depicting the use of specific VR technologies.

The resulting individual storyboards are presented to the group. Participants are encouraged to critically review the applications by explaining when, how and why VR is applied (visualised by the technology frames). The discussions not only inform the facilitator about the background of the storyboards, it may also inspire or trigger other participants to think of new opportunities or challenges.

4. Group storyboard - After discussing the individual storyboards, groups of three to four participants are formed based on similarities in storyboard themes. The groups are asked to discuss, compare and merge their storyboards into a group storyboard. The group storyboards are further detailed, by letting the participants think about the following questions with respect to the application that is visualised in a particular storyboard:

(a) Who are involved in this application?

(b) When in the design process does the application occur?

(c) What is the primary purpose of the application?

(d) Which resources are required for the application?

In addition to providing detailed documentation for the researcher, these questions also trigger more in depth discussions about the application.

5. Wrap-up - During the wrap-up group storyboards are presented to the whole group. The aim of these presentations is to share and discuss the group storyboards, and to reach consensus about which of the group storyboard presents the most interesting storyboard for further use in the case study.

6. Reflection - After the workshop, the facilitator should take time to interpret the resulting storyboards. It was found that the group storyboards often do not depict all the topics that were discussed during the workshop. It is therefore recommended to also include individual storyboards in the analysis of the results. Furthermore, a follow-up refinement meeting may be required to discuss, validate and possibly refine the facilitator's interpretation.

\subsubsection{Prototype development}

During the development phases of the case studies the researcher was responsible for realising a sufficiently functional prototype of the VR application desired for the particular case study. Although these prototypes mainly serve the evaluation of the application, a risk of developing these prototypes in collaboration with (i.e. based on requirements of) company participants is that the prototype is seen as a development track towards a fully functional and usable design tool. This affects both the functionality of the prototype (once the initial requirements were fulfilled, participants came up with new ones) as well as the usability of the prototype (participants expected to be able to deploy the prototype in practice themselves). As already noted in the conclusion of chapter 5 , this risk was particularly present in case study 2 . After conducting the application validation sessions in that case study it was decided to use the application prototype as a basis for a final execution tool.

This risk is nourished by both the researcher and the company participants. The researcher gains experience with software development during the case studies, which allows for the development of more functional and feature rich prototypes. Furthermore, the researcher's desire 
to deliver a sufficiently functional prototype for the application validation easily leads to overrefining the prototype. From the perspective of the company, it is natural for the prototype to elicit new and/or refined requirements regarding the desired VR application. However, it was found that a clear description of the desired application should be established with (and communicated to) the practitioners before the development of a prototype application.

In case study 2, the VR exploration workshop did not directly lead to a concrete application description. In the subsequent refinement sessions, the Virtual Persona application was defined, but within a smaller group of practitioners. Consequently, when reviewing the first prototypes of the application, some of the practitioners did not recognise any of the applications they came up with during the exploration workshop. The lack of a concrete reference (e.g. a desired application defined in the workshop) is likely to be one of the reasons why practitioners in this case study tended to stretch or move the application to more familiar grounds, such as ergonomic analyses or engineering reviews.

Having learned about this risk in case study 2, the following techniques were used effectively to reduce this risk in case study 3 .

- The evolution of the prototype should be within the scope of the application defined in the VR exploration workshop. This application should be clearly defined with, and communicated to case study participants.

- Throughout the case study, the facilitator should repeatedly explain that the role of the prototype is to support the application validation rather than to form a starting point for final tools.

- The limitations of the prototype should be clearly defined and communicated to case study participants. For example, in case study 3 it was decided not to focus on trivial tasks such as importing models or advanced object relations and animations.

Overall, it was found that the use of functional application prototypes significantly contributed the research results. They allowed the design practitioners to experience the applications they envisioned during the exploration workshop, and gain detailed and first-hand experiences in using the applications in practice. As such they also represent exactly what the research is about, namely to experience future use situations in order to elicit feedback from end-users (in this case design practitioners).

\subsubsection{Application validation sessions}

Each case study involved an application validation session in which the application prototype was deployed in a test case for the company. In these sessions, practitioners were asked to verify whether or not the VR application indeed provided the advantages or added value that were expected after defining the application in the exploration workshop. The reliability of these validations depends not only on the quality of the application prototype, but also on the participants involved in the validation, the session's location and the topic of the particular test case.

\section{End-user involvement}

In case studies 1 and 3, in which the VR applications aim to facilitate involvement of end-users, no real end-users were involved. Instead, in case study 1 the practitioners played the part of the 
end-users as they walked through the virtual printshop. In case study 3 , the session did include a multidisciplinary group of stakeholders, but again there were no end-users.

There were various reasons for not directly including end-users in the validation sessions.

- It is not always desirable or feasible to invite end-users to external locations

- Practitioners do not want to expose end-users (i.e. customers) to experimental setups

- End-users are not always expected to be able to contribute to experimental sessions

- Practitioners expect to be able to stand in for end-users in experimental setups

Despite the lack of end-user involvement in the validation sessions, the sessions provided useful feedback and insights into the feasibility of the particular VR applications, for both the researcher and the practitioners. Given the experimental stages in which the research takes place it can be argued that a designer's "educated guess" is sufficiently reliable to decide whether or not a VR application is useful. Furthermore, the designers involved in case study 1 (where enduser involvement would have been most desirable) are quite experienced with UCD, increasing the reliability of their assessment. Nevertheless, it is recommended to involve actual end-users (when applicable) in validating the application.

\section{Location \& Topic}

The application validation session of case study 1 took place at the Virtual Reality Lab of the University of Twente. The validation sessions of case studies 2 and 3 were carried out at the respective company locations. The change of setting was primarily made because of practical reasons; it is easier to invite participants to local sessions rather than requiring them to travel to an external location. Furthermore, the technical setups of the application prototypes did not require specific hardware or facilities.

In addition to these practical benefits, however, it was also found that the sessions were more closely related to the respective companies. In case study 1 the validation sessions had an 'experimental' nature, while the other two validation sessions addressed realistic and topical use cases and involved more in-depth discussions. As the sessions took place within the company, it was easier for the participating companies to include departments that are usually not involved in research and development activities (e.g. manufacturing or assembly engineers). The use of a realistic and recent (or present) test case also contributes to a more in-depth discussion; participants are able to directly relate the session events to real-life situations. It is easier for practitioners to assess the application based on a concrete reference (i.e. a real-life use case) than a fictive situation.

\subsubsection{Cross company evaluation sessions}

The CCE sessions played an important part in the generalisation phases of the research, as they focused on translating the company specific results of one case study to the design context of the other industrial partners involved in the sessions. Making this translation was initially quite difficult. In the first CCE session, the participating companies were introduced to the difference between VR applications and VR tools (the means for realisation). It was emphasised that the aim of the CCE sessions was to translate the application to the respective design domains, and subsequently to review the proposed tools for realising this application. 
In spite of explaining the aim, the participating companies still had difficulties separating applications from tools, typically resulting in discussions primarily about detailed tool aspects, such as the compatibility of file formats, hardware requirements or integration with present tools. While these concerns are obviously relevant and valid, the researcher aimed to discuss them within the context of a specific application. In the other two CCE sessions, the discussion of applications and tools was therefore separated more explicitly by first presenting, experiencing and discussing the VR application, and then presenting and discussing the proposed tools. This resulted in more in-depth discussions about why an application would or would not work for a particular design context, rather than how it would have to be realised. Furthermore, it is expected that the fact that the CCE participants gained experience in collaborating in the CCE sessions as well as the case studies, helped with understanding the difference between applications and tools.

\section{Reliability of tool assessments}

Having translated the initial application into an application for their own design context, the participating designers were asked to assess the feasibility of preparation and/or execution tools that were proposed for the initial application. This requires the participants to make an assessment based on 1) the presentation and demonstration of the initial application, 2) the experiences that the designers from the case study company had with realising the application, and 3) knowledge of their own tool chain, skills and resources. Unlike the designers who were involved in the case study, CCE participants do not have first-hand experience with realising the application.

This limitation reduces the reliability of the conclusion that the applications can be realised within the design contexts of all companies involved in the CCE sessions. It is an assumption based on the assessment made by the participating practitioners, who were informed about the VR application and the VR tools as well as possible in the time frame of the CCE sessions. Furthermore, given that all the companies were able to identify means for realising the applications in their own case studies, it is likely that they are able to make a reliable assessment for the translated applications. However, a more reliable conclusion could be obtained by involving the companies in a more elaborate deployment of the application within the various design contexts, as also done in the individual case studies.

\subsection{Conclusion}

The overall structure of the research approach allowed for an efficient execution of individual case studies and collaborative cross-company evaluation sessions. The sequential involvement of industrial partners enabled the participating practitioners to explore potential VR applications without being (positively or negatively) influenced by external factors such as other companies or the use of specific software or hardware.

Based on the reflection presented in section 8.1, the following is concluded with respect to the research approach.

- Involving industrial partners in a sequential order allows the researcher to iteratively improve the methods used in each iteration. Several drawbacks of this approach, however, should be taken into account. It is important to manage the commitment of the companies throughout the research, especially during the time they are not actively involved in a case study. Furthermore, learning effects can occur when company specific results are 
shared between case studies. In spite of these drawbacks the researcher recommends the use of a sequential approach, especially when the development and evaluation of research methods is desired.

- Active involvement of design practitioners in the research provides relevant insights, but it can also affect the focus of the research. The diversity of the participating practitioners may require the researcher to change focus between case studies. In the current research, the lack of experience with $U C D$ in companies $B$ and $C$ required the researcher to primarily focus on UCD, rather than UCD and VR. Although it is not always feasible for design researchers to 'select' participating departments or practitioners, it is recommended to conduct a preliminary study to assess the current state of (in this case) UCD and VR within the company, and take these insights into account during the research.

- The current research gathered both qualitative and quantitative data. The conclusions of the research have been based primarily on the qualitative data, but are verified and supported by the insights gained from quantitative post-session evaluation forms.

Section 8.2 discussed the implementations of the methods that supported the research approach. Based on the reflection on these methods it is concluded that the strength of the research approach lies in the exploration and identification of VR applications that facilitate UCD activities. The VR exploration workshop and the development and validation of functional prototypes are key activities in this process and demonstrate how design practitioners can effectively be involved in researching new and fairly complex design supports. Furthermore, the lessons learned from the current research provide design researchers with concrete guidelines regarding the organisation of participatory workshops, the development of functional prototypes and the use of these prototypes in validation sessions.

The cross-company evaluation sessions provided insights into how the VR applications can be translated to other design domains. However, the conclusions regarding the realisation of these applications should be considered to be well informed assumptions, as the actual realisation of the applications in the setting of the other companies was not part of the CCE sessions. In future work, these assumptions could be verified by letting the companies realise the applications themselves, as done in the individual case studies. 



\section{Conclusion}

The research presented in this thesis investigated the feasibility of VR as a means to facilitate UCD activities in the early stages of the product design process. As explained in chapter 2 , the research focuses on the early stages of the product design process because this is where UCD activities have the most significant impact. However, to make VR an effective support in these stages of the process, the technology has to be accessible to as well as usable by product designers. In chapter 3, a research approach was presented that addresses both the application of VR in early stage UCD activities and the realisation of these applications by product designers.

Chapters 4, 5 and 6 presented three case studies in which this approach was implemented to identify, deploy and evaluate various VR applications. In chapter 7 the results of the case studies were shared and discussed with the companies that were involved, leading to the following conclusions:

1. In the early stages of the product design process, VR facilitates UCD activities by providing an interactive and integral virtual representation of future use situations in which product concepts can be generated, presented or evaluated.

2. Within this scope of applications, low-end off-the-shelf VR techniques and low fidelity models provide sufficient means for realising these virtual representations.

3. The main challenge for designers to deploy such applications lies in

(a) the identification of an effective VR application and

(b) the selection of appropriate means to realise this applications.

Based on these conclusions the objective of this research, which was to provide insights in the feasibility of VR as means to facilitate early stage UCD activities, has been achieved; it was shown that VR can effectively facilitate UCD activities by providing a virtual representation of future use situations. These applications can be realised by designers themselves, once they have been provided with appropriate preparation and execution tools. Although the realisation itself does not require external support, the preliminary definition of the application and the selection of tools benefit from support (internally or by an external expert) in the form of an exploration workshop and a set of practical selection guidelines respectively.

The following two subsections further elaborate on this conclusion. Section 9.3 discusses the validity of the results and section 9.4 presents recommendations for future work.

\subsection{Facilitating UCD through VR}

VR provides a means to create an interactive and integral representation of future use situations. The virtual representation of future use situations facilitates communication between designers 
and stakeholders that enables uninformed stakeholders (e.g. end-users) to quickly understand product concepts, and to interpret the product's interactions with a use context or the end-user itself. Even without directly involving end-users, the virtual presence of an end-user in the virtual representation can help designers think from the perspective of the end-user, as for instance seen in the Virtual Persona application.

The main contribution that VR offers to early UCD activities is that it allows designers to create these representations without the need for detailed design information. The case studies carried out in this research have shown that low-end VR techniques and low fidelity models of the use context and the product concept provide sufficient means for designers and stakeholders to effectively conduct UCD activities. The maximum return of investment is expected to be found in the early stages of the PDP; relevant insights from (or about) end-users can be obtained without significant investments in e.g. high fidelity models or VR techniques.

\subsection{Realisation of VR applications}

The research also investigated the realisation of VR applications by design practitioners. In chapter 2 it was argued that particularly for early stage design activities, designers need to be in charge of the preparation and execution of design activities rather than being dependent on external resources.

In the case studies and the subsequent cross company evaluation sessions, the tools needed for preparation (e.g. creating 3D models) and for execution of the applications (e.g. providing a $3 \mathrm{D}$ walkthrough functionality) were investigated. While it was originally expected that the main bottleneck for designers to realise VR applications would be the accessibility and usability of these tools, it was found that once a clear definition of the desired VR application had been established, practitioners had few difficulties with using either their present tools or newly acquired tools for the realisation of the application.

Based on this insight, section 7.3 introduced an approach to support designers with the definition of a useful VR application and the selection of appropriate tools for the realisation of this application.

- Application Definition - The application definition aims to explore and identify VR applications that facilitate early stage UCD activities in a given design domain. The VR exploration workshop, which has been outlined in chapter 3 , implemented in three case studies and reviewed in chapter 8 , is considered to provide effective support for this step.

- Tool Selection - The tool selection step aims to identify the preparation and execution tools needed to realise the application defined in the first step. Although is not supported by a concrete workshop or method, practical guidelines which were derived from the cross company evaluation sessions have been presented in section 7.3.

As noted in section 7.3, although both the application definition and the tool selection steps can benefit from external expertise in the field of VR, the actual realisation and use of the final VR application do not require external support.

\subsection{Validity}

In chapter 8 a reflection on the methods and their implementation in the case studies and crosscompany evaluations was conducted to discuss the reliability of the results of the research. In 
this section, the scope in which these results are considered valid is outlined based on the following three aspects.

\subsubsection{Industrial contexts}

The research involved industrial partners from various design domains, with various organisational structures and different levels of experience with VR and UCD. A similarity between the companies is that they are all involved in designing complex interactive systems, ranging from professional printers to food processing machines and transportation vehicles. It was shown that each company has its own approach towards realising proposed VR applications, affected by e.g. existing tools, skills, budgets or attitude regarding UCD. The core function of the VR applications, however, which is to provide an integral and interactive representation of future use situations, addresses a relevant challenge in all the development processes involved in the research.

Given these considerations, it is expected that the research results are at least valid for industrial contexts involved in the development of complex interactive systems. The applicability of the realisation approach presented in section 7.3 has been proven in this domain. However, as the research provides concrete support for the identification, realisation and evaluation of VR applications in a specific design domain, the boundaries of this scope can be further explored.

\subsubsection{Position in product design process}

The stage in the product design process is an important factor that should be taken into account when interpreting the results of this research. The current research addresses the early stages of the product design process, including all design activities up to the point of a concept freeze. Within this phase of the PDP, the research deployed VR as a communication tool; the main role of $V R$ is to facilitate communication between designers and stakeholders about the product by providing a representation of future use situations. It was shown that this in turn facilitates design activities such as concept presentation, generation or evaluation. As explained in section 7.2.3, the added value of using VR in this manner is expected to decrease as the PDP progresses and more detailed design information is gathered. Consequently, a turning point exists where different communication or design tools will be more useful. The position of this turning point was illustrated in case study 2, where the focus of design activities changed from gathering information based on coarse models to validating information based on more detailed models.

\subsubsection{Range of technologies}

As explained in chapter 1 and 2 , the current research focused on the use of VR techniques to facilitate early stage UCD activities. As the researcher primarily used low-end and off the shelf VR techniques to achieve this, the conclusion is only valid with respect to this limited range of VR techniques. It was shown that such techniques, after being introduced and explained to designers, can be deployed without external expertise, which is considered to be an important requirement for UCD tools.

It is expected that novel or complex VR techniques are more difficult to deploy without sufficient technical expertise. Furthermore, it should be noted that this conclusion does not imply that high-end techniques or high-fidelity representations could not support early stage UCD activities. However, based on the present research results it is expected that the added value of using high-end techniques instead of low-end techniques is minimal. 


\subsection{Final thoughts \& future work}

In spite of what was achieved and demonstrated in the current research, the deployment of VR in the early stages of a design process remains challenging in many ways. Apart from the obvious technical aspects, one of the most challenging aspects encountered in the research was to face expectations and preconceptions regarding VR.

As found in the field study presented in chapter 2, the awareness of what VR is and how it could contribute to early stage design activities is generally quite limited; VR is expected to involve high-end hardware and software and high fidelity product models. Early stage design activities, on the other hand, involve rough sketches, few 3D models and a lot of design 'freedom'. From that perspective, VR is considered too complex, difficult to use and too restrictive in terms of design freedom. Conducting the case studies with three very different industrial partners eventually led to a better understanding of how VR could facilitate early stage UCD activities.

It is argued that the key to understanding the role of VR in the context of early stage UCD activities is to consider VR as a means to facilitate communication. Similar to how an animation, sketch or foam model represents a future product, context or use situation, VR can be used to create virtual representations of future use situations. This representation in turn supports design activities such as concept generation, presentation or evaluation. However, rather than claiming that VR will reduce the time it takes to carry out these activities, reduce the costs or immediately result in first-time-right product concepts, it should be stressed that the added value is in the fact that it adds a new perspective on existing information, and possibly helps with eliciting new (or more detailed) information from end-users. As such, it should be seen as an addition to existing communication techniques, including sketches, scenarios, storyboards and physical prototypes.

The researcher therefore recommends to use VR to improve the ability to provide end-users (or any external stakeholder) with a representation of future use situations. This means that it should be deployed in stages in which sketches, scenarios, storyboards and coarse product models are currently used to achieve this.

\subsubsection{Future work}

As $V R$ is a relatively new technique in the context of the early stage UCD activities, it is important to further explore the role of VR in this setting. One of the areas that requires further investigation is the realisation of the translated VR applications in various design domains. As explained in section 8.2.4, the cross-company evaluation sessions primarily discussed the translation of the applications. The assessment of issues regarding the realisation of these applications is relatively unexplored, and could be elaborated by conducting 'tool workshop sessions' with design practitioners, as was done in the case studies.

Another important question resulting from the current research is how much support design practitioners need to execute the approach for the definition of VR applications and the selection of appropriate tools. While the application definition is supported by a concrete method, namely the VR exploration workshop, the tool selection consists of the guidelines presented in chapter 7.3. The adoption of VR as a technique to facilitate early stage UCD activities would benefit from an approach that can be executed by designers themselves. It is expected that especially the tool selection step requires additional efforts to achieve this. The development of a concrete support tool for selecting appropriate VR techniques, for instance in the form of a small booklet or website (which would be easier to keep up to date), would be beneficial. 
Lastly, as concluded in section 9.3 , it is expected that the added value of using high-end techniques instead of low-end techniques to facilitate UCD activities is minimal. While low-end and off-the-shelf techniques provide attractive means, design practitioners sometimes already use high-end software and hardware in other stages of the PDP which could possibly justify their use in the earlier stages. Furthermore, the current application prototypes have not been exposed to the company's end-users; end-users possibly have a different perception or expectation of fidelity than design practitioners. It is therefore recommended to validate this assumption in future work.

\section{Recommendations for design practice}

The research presented in this thesis has shown that even low-end and off the shelf VR techniques can be used to successfully facilitate UCD activities. Therefore, the overall recommendation for design practitioners who are interested in this topic is simply to start trying. Depending on the starting point of design practitioners (in terms of experience with VR and/or UCD), the research provides several concrete leads that guide this process:

- Practitioners who are interested in exploring the potential advantages of using VR in their PDP are advised to start with the VR exploration workshop. This workshop has been carried out several times (inside and outside the scope of the current research) and proven to be an effective and efficient method for exploring the opportunities of VR for a particular design context. Regardless of the company's current state of user centred design or current use of VR techniques, it typically results in concrete VR application scenarios.

- The VR exploration workshop is expected to be most useful when design practitioners are uncertain about which design task or activity to facilitate. Practitioners who already have a clearly defined idea or clear focus on what kind of application is to be developed can use the tool selection guidelines (see section 7.3.2) to select appropriate preparation and execution tools to realise the desired application.

- Practitioners who are specifically interested in further exploring the use of the applications that were developed within the current research can use the prototypes as a starting point for experiments and further development. The application prototypes are available via the REPAR project website ${ }^{1}$.

The overview of future work and recommendations indicates that there is still much to be done for VR to become a common technique in UCD activities. The current research provides sufficient grounds for design researchers to further explore this field, as well as concrete means for design practitioners to 'simply get started' with it.

\footnotetext{
${ }^{1}$ See http://www.repar-project.com
} 



\section{Bibliography}

Balcisoy, S., Kallmann, M., Fua, P., and Thalmann, D. (2000). A framework for rapid evaluation of prototypes with augmented reality. In Proceedings of the ACM symposium on Virtual reality software and technology, pages 61-66, Seoul, Korea. ACM.

Ballagas, R., Ringel, M., Stone, M., and Borchers, J. (2003). iStuff: a physical user interface toolkit for ubiquitous computing environments. In Proceedings of the SIGCHI conference on Human factors in computing systems, pages 537-544, Ft. Lauderdale, Florida, USA. ACM.

Bannon, L. (1991). From human factors to human actors: The role of psychology and humancomputer interaction studies in system design. Design at work: Cooperative design of computer systems, page $25-44$.

Bernsen, N. O., Dybkjær, H., and Dybkjær, L. (1994). Wizard of oz prototyping: How and when. Proc. CCl Working Papers Cognit. Sci./HCl, Roskilde, Denmark.

Blach, R. (2008). Virtual reality technology - an overview. In Talaba, D. and Amditis, A., editors, Product Engineering, pages 21-64. Springer Netherlands.

Blomkvist, S. (2002). The user as a personality. In Using Personas as a tool for design. Position paper for the course workshop "Theoretical perspectives in Human-Computer Interaction".

Bødker, S. (2000). Scenarios in user-centred design-setting the stage for reflection and action. Interacting with Computers, 13(1):61-75.

Bordegoni, M. and Cugini, U. (2006). Haptic modeling in the conceptual phases of product design. Virtual Reality, 9(2):192-202.

Bowman, D. A., Gabbard, J. L., and Hix, D. (2002). A survey of usability evaluation in virtual environments: Classification and comparison of methods. Presence: Teleoperators \& Virtual Environments, 11(4):404-424.

Brandt, E. (2006). Designing exploratory design games: a framework for participation in participatory design? In Proceedings of the ninth conference on Participatory design: Expanding boundaries in design - Volume 1, pages 57-66, Trento, Italy. ACM.

Brandt, E. and Grunnet, C. (2000). Evoking the future: Drama and props in user centered design. In Proceedings of Participatory Design Conference (PDC 2000), page 11-20.

Bruno, F. and Muzzupappa, M. (2010). Product interface design: A participatory approach based on virtual reality. International Journal of Human-Computer Studies, 68(5):254-269. 
Buskermolen, D. O. and Terken, J. (2012). Co-constructing stories: a participatory design technique to elicit in-depth user feedback and suggestions about design concepts. In Proceedings of the 12th Participatory Design Conference: Exploratory Papers, Workshop Descriptions, Industry Cases - Volume 2, PDC '12, page 33-36, New York, NY, USA. ACM.

Calde, S., Goodwin, K., and Reimann, R. (2002). SHS orcas: the first integrated information system for long-term healthcare facility management. In Case studies of the CHI2002: AIGA Experience Design FORUM, CHI '02, page 2-16, New York, NY, USA. ACM.

Carmichael, A., Newell, A. F., Dickinson, A., and Morgan, M. (2005). Using theatre and film to represent user requirements. In Proceeding of Include Conference Royal College of Art, London, volume 5.

Carroll, J. M. (1995). Scenario-based design: envisioning work and technology in system development. John Wiley and Sons, New York.

Cobb, S. and Sharkey, P. (2007). A decade of research and development in disability, virtual reality and associated technologies: Review of ICDVRAT 1996-2006. IJVR, 6:51-68.

Cobb, S. V., D'Cruz, M. D., and Wilson, J. R. (1995). Integrated manufacture: A role for virtual reality? International Journal of Industrial Ergonomics, 16(4-6):411-425.

Crosier, J. K., Cobb, S., and Wilson, J. R. (2002). Key lessons for the design and integration of virtual environments in secondary science. Computers \& Education, 38(1):77-94.

DIS, I. (2010). 9241-210: 2009. ergonomics of human system interaction-part 210: Humancentred design for interactive systems (formerly known as 13407). International Organization for Standardization (ISO). Switzerland.

Dix, A. and Gongora, L. (2011). Externalisation and design. In Procedings of the Second Conference on Creativity and Innovation in Design, DESIRE '11, page 31-42, New York, NY, USA. ACM.

Eastgate, R. (2001). The structured development of virtual environments: Enhancing functionality and interactivity. University of Nottingham.

Fencott, C. (2004). A methodology of design for virtual environments. In Sanchez-Segura, M., editor, Developing future interactive systems, pages 66-89. Idea Group, London.

Garde, J. and van der Voort, M. C. (2012). Co-designing better work organization in healthcare. Advances in Human Aspects of Healthcare, 10:23-32.

Gould, J. (1988). How to design usable systems. Martin Helander (Ed.) Handbook of HumanComputer Interaction, North-Holland: Elsevier, page 757-789.

Greenbaum, J. M. and Kyng, M. (1991). Design at work: Cooperative design of computer systems. CRC Press, Hillsdale, NJ, 1 edition.

Grudin, J. and Pruitt, J. (2002). Personas, participatory design and product development: An infrastructure for engagement. In Proc. PDC, volume 2002, page 7 th. 
Gulliksen, J., Goransson, B., Boivie, I., Blomkvist, S., Persson, J., and Cajander, A. (2003). Key principles for user-centred systems design. Behaviour \& Information Technology, 22(6):397-409.

Halskov, K. and Dalsgard, P. (2006). Inspiration card workshops. In Proceedings of the 6th conference on Designing Interactive systems, pages 2-11, University Park, PA, USA. ACM.

Huang, G. Q. (2002). Web-based support for collaborative product design review. Computers in Industry, 48(1):71-88.

livari, J. and livari, N. (2011). Varieties of user-centredness: an analysis of four systems development methods. Information Systems Journal, 21(2):125-153.

Jimeno, A. and Puerta, A. (2007). State of the art of the virtual reality applied to design and manufacturing processes. The International Journal of Advanced Manufacturing Technology, 33(9):866-874.

Jung, T., Gross, M. D., and Do, E. Y.-L. (2002). Annotating and sketching on 3D web models. In Proceedings of the 7th international conference on Intelligent user interfaces, IUI '02, page 95-102, New York, NY, USA. ACM.

Kan, H. Y., Duffy, V. G., and Su, C.-J. (2001). An internet virtual reality collaborative environment for effective product design. Computers in Industry, 45(2):197-213.

Karat, J. (1996). User centered design: quality or quackery? In Interactions, volume 3, page 18-20. ACM, New York, 4 edition.

Kaulio, M. A. (1998). Customer, consumer and user involvement in product development: A framework and a review of selected methods. Total Quality Management, 9(1):141.

Kaur, K. (1998). Designing virtual environments for usability. Centre for Human-Computer Interface Design, City University, London.

Kitzinger, J. (1995). Introducing focus groups. BMJ: British Medical Journal, 311(7000):299302.

Koen, P., Ajamian, G., Burkart, R., Clamen, A., Davidson, J., D'Amore, R., Elkins, C., Herald, K., Incorvia, M., Johnson, A., et al. (2001). Providing clarity and a common language to the" fuzzy front end". Research-Technology Management, 44(2):46-55.

Krishnan, V. and Ulrich, K. T. (2001). Product development decisions: A review of the literature. Management Science, 47(1):1-21.

Kuutti, K., Battarbee, K., Säde, S., Mattelmäki, T., Keinonen, T., Teirikko, T., and Tornberg, A. (2001). Virtual prototypes in usability testing. In Hawaii International Conference on System Sciences, volume 5, page 5029, Los Alamitos, CA, USA. IEEE Computer Society.

Lee, J. C., Avrahami, D., Hudson, S. E., Forlizzi, J., Dietz, P. H., and Leigh, D. (2004). Proceedings of the 5th conference on designing interactive systems: processes, practices, methods, and techniques. pages 167-175, Cambridge, MA, USA. ACM.

Lenne, D., Thouvenin, I., and Aubry, S. (2009). Supporting design with 3D-annotations in a collaborative virtual environment. Research in Engineering Design, 20(3):149-155. 
Maguire, M. (2001). Methods to support human-centred design. International Journal of Human Computer Studies, 55(4):587-634.

May, A. and Carter, C. (2001). A case study of virtual team working in the european automotive industry. International Journal of Industrial Ergonomics, 27(3):171-186.

Miedema, J. (2010). Synthetic Environments in Design Processes. PhD thesis, University of Twente, Enschede.

Milgram, P. and Kishino, F. (1994). A taxonomy of mixed reality visual displays. IEICE TRANSACTIONS on Information and Systems, 77(12):1321-1329.

Nielsen, J. (1994). Usability engineering. Academic press Itd., London.

Nobelius, D. and Trygg, L. (2002). Stop chasing the front end process - management of the early phases in product development projects. International Journal of Project Management, 20(5):331-340.

Norman, D. A. (1986). Cognitive engineering. In User centered system design, page 31-61. Lawrence Erlbaum Associates, Hillsdale, NJ.

Norman, D. A. (1988). The psychology of everyday things. Basic books.

Norman, D. A. (2002). The Design of Everyday Things. Basic Books.

Norman, D. A. and Draper, S. W. (1986). User Centered System Design; New Perspectives on Human-Computer Interaction. L. Erlbaum Associates Inc.

Ottosson, S. (1998). Qualified product concept design needs a proper combination of pencilaided design and model-aided design before product data management. Journal of Engineering Design, 9(2):107.

Ottosson, S. (2002). Virtual reality in the product development process. Journal of Engineering Design, 13(2):159-172.

Schreiber, M., von Wilamowitz-Moellendorff, M., and Bruder, R. (2009). New interaction concepts by using the wii remote. Human-Computer Interaction. Novel Interaction Methods and Techniques, page 261-270.

Sutherland, I. E. (1968). A head-mounted three dimensional display. In Proceedings of the December 9-11, 1968, fall joint computer conference, part I, pages 757-764, San Francisco, California. ACM.

Tideman, M., van der Voort, M., and van Houten, F. (2008). A new product design method based on virtual reality, gaming and scenarios. International Journal on Interactive Design and Manufacturing, 2(4):195-205.

Travis, D. (2011). ISO 13407 is dead. long live ISO 9241-210! http://www.userfocus.co.uk/articles/iso-13407-is-dead.html, accessed 2013-08-05.

Ulrich, K. T. and Eppinger, S. D. (1995). Product design and development, volume 384. McGraw-Hill New York. 
Urnes, T., Weltzien, A., Zanussi, A., Engbakk, S., and Rafn, J. K. (2002). Pivots and structured play: stimulating creative user input in concept development. In Proceedings of the second Nordic conference on Human-computer interaction, pages 187-196, Aarhus, Denmark. ACM.

van der Bijl-Brouwer, M. (2012). Exploring usability: design for dynamic and diverse use situations. PhD thesis, University of Twente, Enschede.

Vavoula, G. N., Sharples, M., and Rudman, P. D. (2002). Developing the'Future technology workshop'method. In Proceedings of the International Workshop on Interaction Design and Children, Aug, page 28-29.

Veryzer, R. W. and Borja de Mozota, B. (2005). The impact of user-oriented design on new product development: An examination of fundamental relationships. Journal of Product Innovation Management, 22(2):128-143.

Visser, F. S., Stappers, P. J., Van der Lugt, R., and Sanders, E. B. (2005). Contextmapping: experiences from practice. CoDesign, 1(2):119-149.

Vredenburg, K., Mao, J.-Y., Smith, P. W., and Carey, T. (2002). A survey of user-centered design practice. pages 471-478, Minneapolis, Minnesota, USA. ACM.

Wang, L., Shen, W., Xie, H., Neelamkavil, J., and Pardasani, A. (2002). Collaborative conceptual design—state of the art and future trends. Computer-Aided Design, 34(13):981-996.

Weidlich, D., Cser, L., Polzin, T., Cristiano, D., and Zickner, H. (2009). Virtual reality approaches for immersive design. International Journal on Interactive Design and Manufacturing (IJIDeM), 3(2):103-108.

Wright, T. E. and Madey, G. (2009). A survey of technologies for building collaborative virtual environments. The International Journal of Virtual Reality, 8(1):53-66.

Ye, J., Campbell, R., Page, T., and Badni, K. (2006). An investigation into the implementation of virtual reality technologies in support of conceptual design. Design Studies, 27(1):77-97. 



\section{Publications}

1. Thalen, J.P. \& Garde, J.A. (2013). Capturing use: user involvement and participatory design. In: Bont, C. de, F. Smulders, M.C. van der Voort, R. Schifferstein \& E. den Ouden (eds) (2013). Advanced design methods for successful innovation - Recent methods from design research and design consultancy in the Netherlands, pp 33-55. Delft: Design United.

2. Thalen, J.P. \& van der Voort, M.C. (2013) The VR Exploration Workshop, workshop for the Design through exploration symposium, Utrecht, the Netherlands

3. Thalen, J.P. \& van der Voort, M.C. (2013). Facilitating user-centred design through virtual reality. In: Terken, J., J.B. Martens \& M.C. van der Voort (eds). Design through Exploration - the REPAR project, pp 15-24. Eindhoven: Eindhoven University of Technology.

4. Thalen, J.P. \& van der Voort, M.C. (2012). The Virtual Printshop: A case study on using Virtual Reality in early stages of user-centered product design. SBC Journal on 3D Interactive Systems, Vol 3, No 3, pp 2-11.

5. Thalen, J.P. (2012) Using Virtual Reality in User Centered Design, workshop for the fourth Wold Usability Day, Utrecht, the Netherlands

6. Thalen, J.P. \& van der Voort, M.C. (2012). Facilitating User Involvement in Product Design Through Virtual Reality, Virtual Reality - Human Computer Interaction, Xin-Xing Tang (Ed.), ISBN: 978-953-51-0721-7, InTech

7. Thalen, J.P. (2012). Ontwerpen met Virtual Reality, in Tijdschrift voor Ergonomie, Vol 3, No 3, pp 17-22. ISSN 0921-4348

8. Damgrave, R.G.J., Lutters, D. \& Thalen, J.P. (2012). Selecting Virtual Reality Tools in relation with their use context, in proceedings of the 4th International Conference on Changeable, Agile, Reconfigurable and Virtual production, Montreal, Canada, 2-5 October 2011. (pp 269-274). Springer.

9. Thalen, J.P., van der Voort, M.C. (2011). Creating Useful, Usable and Accessible VR Design Tools: An EUD-Based Approach. In: Costabile, M.F., Dittrich, Y., Fischer, G. \& Piccinno, A (eds). End-User Development. LCNS 6654. pp 399-402. Springer Berlin Heidelberg 
10. Thalen, J.P. \& van der Voort, M.C. (2011). User Centred Methods for Gathering VR Design Tool Requirements. in proceedings of Joint Virtual Reality Conference, Nottingham, UK: EUROVR

11. Ozcelik, D., Quevedo-Fernandez, J., Thalen, J.P. \& Terken, J.M.B. (2011). Engaging users in the early phases of the design process: Attitudes, concerns and challenges from industrial practice, in proceedings of the 2011 Conference on Designing Pleasurable Products and Interfaces, (pp 1-8). New York: ACM.

12. Ozcelik, D., Quevedo Fernandez, J., Thalen, J.P. \& Terken, J.M.B. (2011). On the development of electronic design tools and associated guidelines for supporting the early stages of the design Process. DESIRE '11 Proceedings of the Second Conference on Creativity and Innovation in Design, October 19-21, 2011, Eindhoven, The Netherlands, (pp 115-126). ACM.

13. Thalen, J.P. (2011). Using Blender for Rapid Virtual Reality Prototyping, presentation at the 10th Annual Blender Conference, Amsterdam, the Netherlands

14. Thalen, J.P. \& van der Voort, M.C. (2011). Turning VR into something useful. In: Roozenburg, N.F.M., L.L. Chen \& P.J. Stappers (Eds.). Diversity \& Unity: Proceedings of World Conference on Design Research 2011, No. 738. Delft, the Netherlands: IASDR 
A Case Study 1 Data 


\section{A.1 Application validation}

The application validation session of Case study 1 consists of an interactive part during which company representatives (table A.1 lists the participants involved in the session) use different manifestations of the Virtual Printshop in a mini use case, and a review part during which the researcher discusses the different forms of the Virtual Printshop with the participants. Although the review was intended to be guided by the evaluation forms included in appendix A.1.1, during the session an unstructured discussion form was favoured by the participants. The transcript of this session, including the interactive part as well as the subsequent discussion has been analysed using an open coding scheme. The results of this analysis are presented in appendix A.1.2.

\begin{tabular}{ll}
\hline Label & Function \\
\hline P1 & Usability Engineer \\
P2 & Visual Designer \\
P3 & Product Designer \\
P4 & Digital Prototyper \\
R1 & REPAR researcher \\
R2 & REPAR researcher \\
FAC & Facilitator \\
\hline
\end{tabular}

Table A.1 List of participants and their labels.

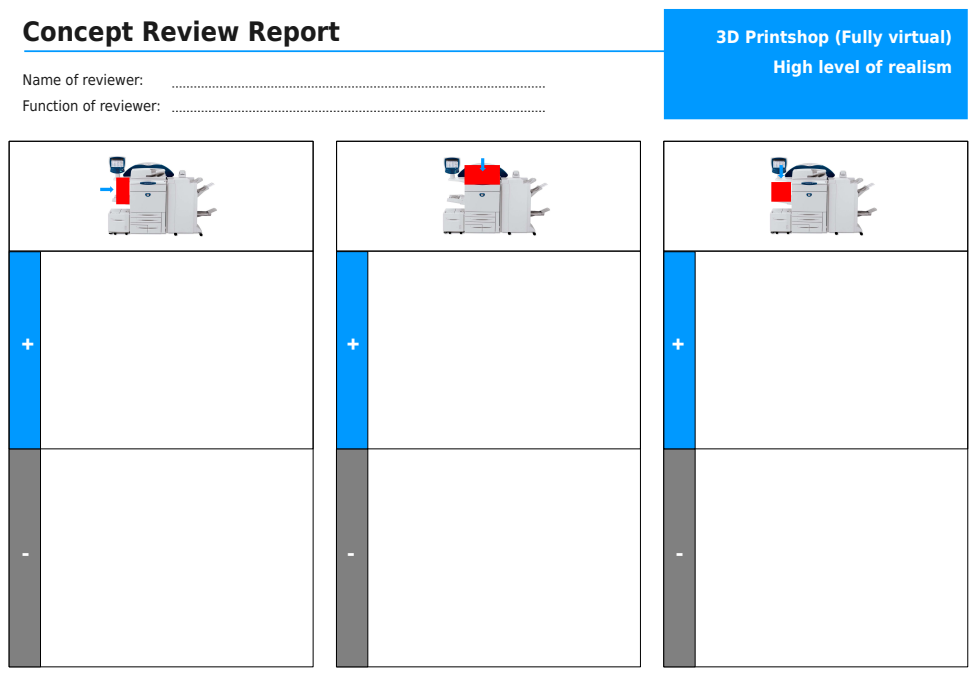

Figure A.1 While using the Virtual Printshop, participants filled out these evaluation forms for the three paper tray design concepts. 


\section{A.1.1 Application validation form}

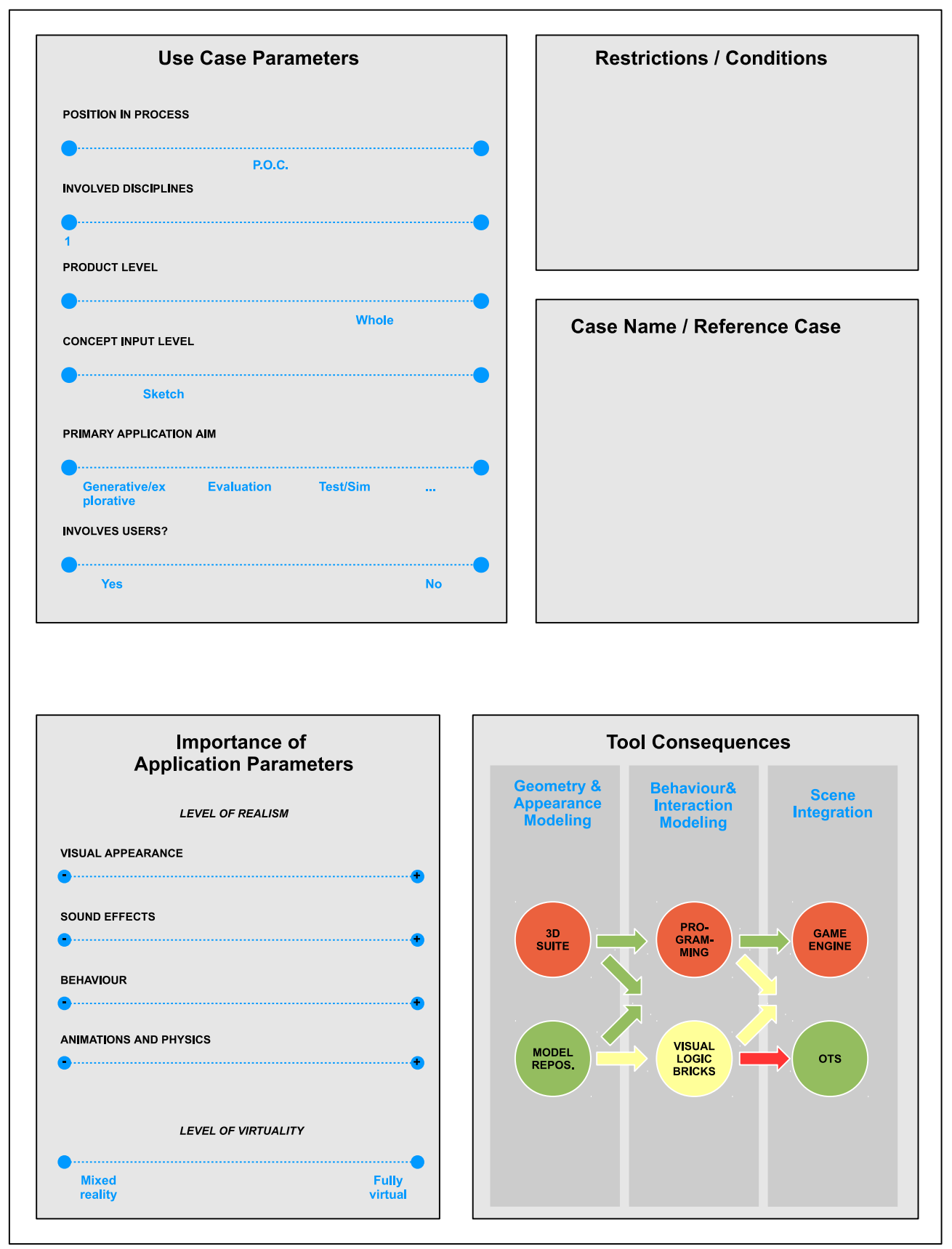




\section{A.1.2 Application validation results}

\section{Virtual Printshop}

\begin{tabular}{|c|c|c|}
\hline \multicolumn{3}{|c|}{ A. 3D Printshop } \\
\hline \multirow[t]{2}{*}{ 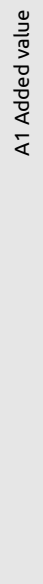 } & $\begin{array}{l}\text { A1.1 Design } \\
\text { support }\end{array}$ & $\begin{array}{l}\text { 1. It can improve the feeling of imagining owning the machine, and predicting responses or issues. } \\
\text { Agreement that the real added value is here in showing the product in a realistic and familiar } \\
\text { context, and simulate daily life. } \\
\text { 2. P4 says that printer activity is difficult to see from a distance. They notice this in the } \\
\text { environment, but he is triggered during playing (and other ideas like this may pop up) to notice } \\
\text { this and think of solutions. } \\
\text { 3. P1 says it is very effective for visualising specific problems with specific printers. } \\
\text { 4. It also helps as an inspirational tool for comming up with new ideas. } \\
\text { 5. P1 concludes that you can evaluate alternatives, but also come up with new ideas (generative vs } \\
\text { evaluation). } \\
\text { 6. The questions to be answered with this setup should be about context, not the printer specifics } \\
\text { (P4). The setup is really good in showing the workflow, and the effects of specific tasks on the } \\
\text { context, such as the queue. } \\
\text { 7. Benefits of this setup are primarily expected for project members, internal communication. }\end{array}$ \\
\hline & A1.2 Insights & $\begin{array}{l}\text { 1. It does show something about the product in context, like the lack of space. But this was also } \\
\text { clear before doing the evaluation in VR. } \\
\text { 2. There is the idea to change the room layout, because they see it's not optimised as it is now. The } \\
\text { current implementation does give a trigger about this, but it could also have done without } \\
\text { (before the session). } \\
\text { 3. It should be more about spatial things such as distance to paper stock, workflow performance, } \\
\text { etc. Contextual items such as the queue are important for that as well. } \\
\text { 4. Concluding: workflow focus instead of physical aspects of concepts, the level of realism of } \\
\text { context is less important than the level of realism of products that are part of the workflow. }\end{array}$ \\
\hline \multirow[t]{2}{*}{ 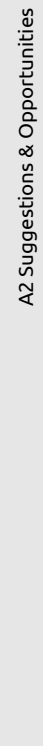 } & $\begin{array}{l}\text { A2.1 Technical } \\
\text { improvements }\end{array}$ & $\begin{array}{l}\text { 1. It is proposed to use a 3rd person view to see people reach for a certain tray concept (height, } \\
\text { force, etc.). } \\
\text { 2. R1 asks if a reference character would help. Response is that a realtime 3rd person view would } \\
\text { be better. } \\
\text { 3. Alternative technologies would make the setup more convincing regarding physical interactions } \\
\text { with concepts. } \\
\text { 4. Other input devices may also make it easier for non-computer users to use a setup like this. } \\
\text { 5. P4 asks if it should then also be multi-user. There is agreement that it would be nice, but not } \\
\text { nessesary (this is about multi-user controlers/input, not other persons walking around in the } \\
\text { virtual environment). } \\
\text { 6. P4 notes that the sound really affects the interaction; in the HF the sound is realistic, but it's } \\
\text { difficult to use as a printing status indicator. In the LF the sound is on or off, which makes it } \\
\text { easier to see printer activity. } \\
\text { 7. P1 responds that it would help if the designer is able to see what the user does (eg 3rd person } \\
\text { view). } \\
\text { 84 asks if the XSense tracking suit could help? FAC explained that the physical space limits the } \\
\text { use in this case, but it could help with tracking body movements when standing still. P4 also } \\
\text { notes some practical limitations when using this with clients. }\end{array}$ \\
\hline & $\begin{array}{l}\text { A2.2 Application } \\
\text { extensions }\end{array}$ & $\begin{array}{l}\text { 1. What would be interesting is to combine a virtual version of an existing environment with new } \\
\text { products. Then put the new product in this context and see how it affects task sequences. This } \\
\text { would be an explorative and generative session, focussing on the workflow and complete } \\
\text { context/environment, not the printer itself. } \\
\text { 2. Pre-prepared concepts (like software) could also be tested in this environment, so it would also } \\
\text { support evaluation tasks. P1 adds again that layout issues can also be easiliy evaluation, like } \\
\text { finding a better position for a printer. } \\
\text { 3. P4 starts about environment friendly workflow design. With a setup like this, you could show } \\
\text { companies their current situation, then replace some of the printers with virtual models of new } \\
\text { printers and explain the differences in environmental footprint, or even let the tool calculate } \\
\text { the footprint. }\end{array}$ \\
\hline \multirow[t]{3}{*}{ 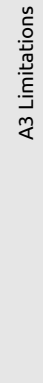 } & $\begin{array}{l}\text { A3.1 Design } \\
\text { support }\end{array}$ & $\begin{array}{l}\text { 1. He notes that in this game it's too simple to open/close the different trays, which is not what } \\
\text { you want. The physical difficulties are not represented in the environment. You don't get the } \\
\text { feeling of having to get into the corner to open/close a tray. } \\
\text { Other thing is that P4 has no idea of the height of the document feeder is too high or ok, from } \\
\text { an ergonomics perspective. } \\
\text { 3. Current implementation does not answer questions related to ergonomics/physical properties. } \\
\text { 4. Agreement about the statement that this implementation is better for workflow analysis rather } \\
\text { than ergonomics or physical aspects. }\end{array}$ \\
\hline & A3.2 Technical & $\begin{array}{l}\text { 1. Should perform the operating yourself, like opening the tray. In this implemetnation it's just a } \\
\text { key/mouse click. } \\
\text { 2. P1 notes that in the LF demo it is more difficult to assess object dimensions, because of a lack of } \\
\text { reference. This happens with objects, but not really with the context. }\end{array}$ \\
\hline & A3.3 Other & $\begin{array}{l}\text { 1. A good question is whether or not users would take it serious? They would definately recognise } \\
\text { it, but may not take it serious. }\end{array}$ \\
\hline
\end{tabular}




\section{Virtual Printshop (continued)}

\begin{tabular}{|c|c|c|c|}
\hline \multirow[t]{2}{*}{ 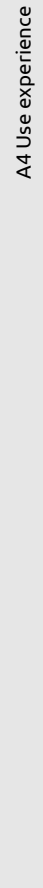 } & A4.1 Controls & $\begin{array}{l}1 . \\
2 . \\
3 .\end{array}$ & $\begin{array}{l}\text { Makes good use of the crouching and zooming features } \\
\text { Has trouble combining the mouse control with keyboard input, but after a minute finds out how } \\
\text { it works. } \\
\text { Has no trouble at all with the mouse and keys, because of past experiences with games (pointed } \\
\text { out by collegues). } \\
\text { Making the controls more difficult is not expected useful because it doesn't help with really } \\
\text { evaluating usability issues (the difficulty is there, but still not related to ergonomics). }\end{array}$ \\
\hline & $\begin{array}{l}\text { A4.3 Task } \\
\text { performance }\end{array}$ & & $\begin{array}{l}\text { Manages to pick up a pack of paper and evaluates the next concept. Succesfully adds paper to } \\
\text { the printer, and inspects the queue afterwards. He gets stuck between the tray and the plotter. } \\
\text { After closing the tray he can walk towards the queue. } \\
\text { P2 notes that she got stuck unexpectedly behind a box, because of the lack of visual cues. } \\
\text { He quickly walks through the evaluation task and then starts exploring the environment. } \\
\text { After the evaluation task she further explores the environment and checks the queue status. }\end{array}$ \\
\hline
\end{tabular}




\section{AR Printshop}

\begin{tabular}{|c|c|c|c|}
\hline \multicolumn{4}{|c|}{ B. AR Printshop } \\
\hline \multirow{2}{*}{$\begin{array}{l}\frac{0}{2} \\
\sum_{0}^{\frac{0}{0}} \\
\frac{d}{0} \\
\frac{0}{0} \\
\frac{0}{\infty}\end{array}$} & B1.1 Design support & & $\begin{array}{l}\text { Participants like the way of switching concept, by just flipping the marker to the next one in the } \\
\text { pile of concepts. } \\
\text { She does like the walking around aspect of it though. }\end{array}$ \\
\hline & B1.2 Insights & & $\begin{array}{l}\text { For some projects, where large designs are involved, this solution would add awereness of } \\
\text { dimensions and scale. This is less user oriented but more on internal communication; make } \\
\text { members aware of real-life implications on the product (e.g. replace 1:1 posters they're using } \\
\text { now). } \\
\text { Mixed reality here is a mix of the virtual objects and a strange environment (the VR lab here). You } \\
\text { actually want a real room and virtual objects. P2 says this way of working is very goal/object } \\
\text { oriented, you go from one object to another. You totally ignore context/environment. }\end{array}$ \\
\hline \multirow{2}{*}{ 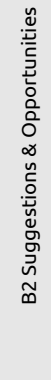 } & $\begin{array}{l}\text { B2.1 Technical } \\
\text { improvements }\end{array}$ & & $\begin{array}{l}\text { Also, especially for this case, the environment should include walls. } \\
\text { Would it help to do some technical changes, towards HMD for instance? Maybe, but in this setup } \\
\text { it's still a wrong mix of reality and virtuality. } \\
\text { P1 adds that the combination with the GUI may also be interesting; use the virtual model to show } \\
\text { effects of what happens in the GUI, such as trays opening, paper comming out, etc. }\end{array}$ \\
\hline & $\begin{array}{l}\text { B2.2 Application } \\
\text { extensions }\end{array}$ & & $\begin{array}{l}\text { What if you use a similar but not totally } 1: 1 \text { layout in the Company A usability lab? Response: this } \\
\text { would create an "Company A printshop", preventing people from feeling at home. This mimicks } \\
\text { the current usability test setups. } \\
\text { It would be more interesting to do it the other way around; do the AR thing at the client's site. } \\
\text { There is a reference to the beam-me up Scotty scenario of the workshop. This could be benefitial } \\
\text { for evaluation and marketing purpose. It also solves the problem of the lack of physical space in } \\
\text { the Company A usability lab (it's smaller than an average printshop). } \\
\text { It should be brought to the client, in their real environment with new products. } \\
\text { One thing would be visual validation of the product in a future context, mainly the appearance } \\
\text { and fitting of the machine in the client's environment. }\end{array}$ \\
\hline \multirow[t]{3}{*}{ 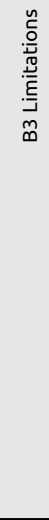 } & B3.1 Design support & & $\begin{array}{l}\text { In the current setup you lack vital visual information about the context. } \\
\text { P3 notes that she's not as involved in the rest of the room (compared to first concepts) because } \\
\text { there are no walls and other context cues. } \\
\text { lt's noted that you only see the objects, not the environment. This has benefits and drawbacks. } \\
\text { While you do physically walk around and experience the environment, the lack of context and the } \\
\text { small view make it difficult to really feel as if 'inside' the print shop. Objects pop up when they're } \\
\text { in range, and only in the small screen. Also this setup forces you to look down to the ground } \\
\text { instead of looking around. } \\
\text { Would AR add fidelity to such a room? Response: then the added value would be limited, because } \\
\text { AR would only add secondary objects, that are not part of the primary test. }\end{array}$ \\
\hline & B3.2 Technical & & $\begin{array}{l}\text { P3 notices that distant markers are not properly recognised which causes a lack of context } \\
\text { awareness. } \\
\text { P1 confirms that the models look smaller than real-life. } \\
\text { The 3D lab was more 'virtual' (complete) than the mixed reality environment. Also caused by the } \\
\text { fact that distant objects do not appear on screen. } \\
\text { The complete environment helps designers to notice problems with visability, distances, etc. In } \\
\text { AR these aspects disappear because you only see one object at a time, not the environment. In } \\
\text { AR you loose the reference for distance and dimensions. }\end{array}$ \\
\hline & B3.3 Other & & $\begin{array}{l}\text { She explains that she expected a pre-recorded (3d) movie of a printshop, and then walk around } \\
\text { in that space. Now she's missing the actual environment, she only sees the object. }\end{array}$ \\
\hline \multirow[t]{3}{*}{ 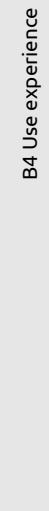 } & B4.1 Controls & & $\begin{array}{l}\text { After task completion the environment is explored by walking around and watching the markers. } \\
\text { There is quick agreement on how to layout the room. The printer concepts are recognised and } \\
\text { put on a pile. } \\
\text { One is holding the tablet while the others are arranging the markers according to instructions of } \\
\text { the participant holding the tablet. } \\
\text { After getting the paper he is able to approach the printer, open the tray and have the paper } \\
\text { added. } \\
\text { Has no problem with paper collection and walking from marker to marker. } \\
\text { A problem arrises with this concept when the tray doens't open because it's out of reach. }\end{array}$ \\
\hline & $\begin{array}{l}\text { B4.2 Level of } \\
\text { realism }\end{array}$ & & $\begin{array}{l}\text { This depends on the aim of the application; if you want feedback about looks, it should look ok. } \\
\text { It's agreed that the entire scale of realism should be supported, aligned with the development } \\
\text { process. }\end{array}$ \\
\hline & $\begin{array}{l}\text { B4.3 Task } \\
\text { performance }\end{array}$ & & $\begin{array}{l}\text { There are some troubles with finding the paper, but then finds the paper pickup region. } \\
\text { Participant has no trouble finding the right markers, and walks around the environment before } \\
\text { carrying out the task. } \\
\text { A problem with the visibility of the paper indicator had to be fixed by FAC to make it work again. } \\
\text { Carries out the task with the same concept as run } 3 \text {, has no problem with finding paper and } \\
\text { adding it to the printer. } \\
\text { Carries out the same evaluation task with the LF version of the AR setup. } \\
\text { Starts the evaluation task but has trouble finding the paper pick-up location. }\end{array}$ \\
\hline
\end{tabular}




\section{Review and discussion}

\begin{tabular}{|c|c|c|}
\hline \multicolumn{3}{|c|}{ C. Review and discussion } \\
\hline \multirow[t]{3}{*}{$\begin{array}{l}\text { C1 Level of } \\
\text { realism }\end{array}$} & C1.1 Visual & $\begin{array}{l}\text { 1. Visual realism is difficult to fill out in the slider because of the distinction between } \\
\text { context quality and product quality. } \\
\text { 2. P4 gives an exampe of required realism for a case where multiple jobs need to be } \\
\text { combined. He states that in such an example jobs need to be recognizable, but not } \\
\text { nessecarily fully realistic. It could be represented in icons as well, as also done in the } \\
\text { demo. } \\
\text { 3. Fully realistic replica's of environments or objects will not be possible anyway. } \\
\text { 4. In behaviour this is the same; it should be recognisable, functional, but not always } \\
\text { real. } \\
\text { 5. However P1 states that this is not always true. Icons are not available in real-life, so } \\
\text { then you still can not test the usability of a complex task. The icons would make the } \\
\text { task easier, but only in the virtual world. P4 replies that this is true, but it still makes } \\
\text { it useful as a learning/training application. FAC responds that this is indeed true, but } \\
\text { outside the scope of the targeted application. P4 agrees and adds that other cases } \\
\text { such as impressing/convincing clients is another example of useful applications. }\end{array}$ \\
\hline & C1.2 Audio & $\begin{array}{l}\text { 1. During the demo's the audio in the low-fi setup was considered more effective, but } \\
\text { less realistic (no sound vs sound). } \\
\text { 2. It is agreed that sound should be better than the low-fi setup today, and more } \\
\text { accurately represent realistic sound circumstances. P2 adds that realistic sound is } \\
\text { quite important. } \\
\text { 3. However, no sound at all would give the same information as realistic sound, as it just } \\
\text { creates 'noise'. } \\
\text { 4. Sound is a nice addition, but for workflow analysis it wouldn't have a lot of impact. P1 } \\
\text { adds that sound is difficult; no sound means no wrong information. If you add sound, } \\
\text { it should be just right to prevent giving the wrong information. Again it depends on } \\
\text { the aim of the setup. } \\
\text { Conclusion is that sound should be either off, or just right, not in between. P4 adds } \\
\text { that it should be spatial sound, matching the visual information. }\end{array}$ \\
\hline & C1.3 Behaviour & $\begin{array}{l}\text { 1. In a workflow analysis you need to be able to see what the user does with an object. } \\
\text { For instance see a user add a print job to the computer. In such a case you would need } \\
\text { to see the GUI, instead of just a blue square for instance. } \\
\text { 2. Users need to be able to see changing status in objects, so see an indication of jobs } \\
\text { arriving, printer status, etc. }\end{array}$ \\
\hline & C1.4 General & $\begin{array}{l}\text { 3. When users are involved the level of realism/fidelity should be higher then when } \\
\text { used in internal cases. However, participants also agree that realism has to reach a } \\
\text { certain level (higher than today's lowest) to achieve triggering problems in the } \\
\text { context. } \\
\text { 4. It is repeated that the realism/quality of the context is less important than realism of } \\
\text { products. It is thought that layout of the environment should be sufficient to } \\
\text { recognise it. } \\
\text { 5. P3 responds that the subject of the test should receive focus and have high fidelity. If } \\
\text { it's about the GUI, moving trays don't matter. If it's about the tray, an interactive GUI } \\
\text { is not needed. } \\
\text { 6. With respect to the level of realism, it's considered important that the context is } \\
\text { recognisable, but doesn't have to be a 1:1 copy of the real environment. } \\
\text { 7. It is expected to take several tries to discover what things should or should not be } \\
\text { included in the environment to achieve recognition with the user. }\end{array}$ \\
\hline $\begin{array}{l}\text { C2 Level of } \\
\text { virtuality }\end{array}$ & C2.1 & $\begin{array}{l}\text { 1. P1 promptly responds that he sees more advantages in the fully virtual setup. Partly } \\
\text { because of some of expected problems with the markers, like how to make them } \\
\text { visible to the users (e.g. HMD, tablet, etc.). } \\
\text { 2. Furthermore, the virtual model basically covers the same advantages as the AR setup, } \\
\text { but also adds more context awareness, and provides a more versatile testing } \\
\text { platform. } \\
\text { 3. Having experienced all four variables, participants indicate a preference for the fully } \\
\text { vritual environment. }\end{array}$ \\
\hline \multirow[t]{2}{*}{$\begin{array}{l}\text { C3 Tool chain } \\
\text { requirements }\end{array}$} & C3.1 Integration & $\begin{array}{l}\text { 1. P3 responds that the modeling part is covered by the product designers, a model is } \\
\text { available for all Company A products. } \\
\text { 2. It is agreed that this part of the tool chain is quite well covered. } \\
\text { 3. If behaviour is required, then they go to the prototypers, and they will use flexible } \\
\text { tools (scripts/programming) to achieve this step as well. } \\
\text { 4. Environment building should be easy, making use of existing Company A models and } \\
\text { external repositories. This envisioned tool chain would form a useful starting } \\
\text { platform for VR at Company A. } \\
\text { 5. Still it would not be a program for daily use. } \\
\text { 6. It will mainly be used in early stages, in later stages it will be replaced by real } \\
\text { prototypes as soon as possible. } \\
\text { 7. Considering the tool chain, the idea is to combine modeling with scene integration to } \\
\text { form a first platform that supports mainly internal communication applications. }\end{array}$ \\
\hline & C3.2 Functionality & 1. P4 states that behaviour should be as configurable as possible, everything should be \\
\hline
\end{tabular}




\section{Review and discussion (continued)}

\begin{tabular}{|c|c|c|}
\hline & $\begin{array}{l}4 . \\
5 . \\
6 .\end{array}$ & $\begin{array}{l}\text { adaptable and configurable. Not everything is used all the time, but depending on } \\
\text { the use case you want to be able to achieve certain things. } \\
\text { P4 says that this (behaviour modelling) should indeed be an Company A design task, } \\
\text { so within the department. } \\
\text { There is agreement on this idea, and a feeling that the modeling and scene } \\
\text { integration steps are already quite well covered by various applications and } \\
\text { databases, such as the Ikea software, the Sims and Google Sketchup. Such tools are } \\
\text { meant for children and should also be applicable here. } \\
\text { It is noted that monitoring options should also be added (overview camera's, etc.). } \\
\text { P4 says that if you want to create something that really helps Company A, it should } \\
\text { be a solution with a lot of possibilities, so behaviour, interaction, visualisation, etc. } \\
\text { P4 imagines a tool that allows regular designers to combine models into a virtual } \\
\text { environment. As soon as you want to bring the environment to life, with audio etc, } \\
\text { then they'd have to go to the model shop and the prototyper will create the fully } \\
\text { interactive environment. } \\
\text { Without that it would be a visualisation/presentation, but with prototyping it would } \\
\text { result in a more interactive application. } \\
\text { Adding behaviour (through flexible scripting/programming) will also add } \\
\text { evaluation/testing applications to the tool chain. This means that scene integration } \\
\text { should be as simple as possible, while behaviour modeling can make use of more } \\
\text { flexible complex tools. } \\
\text { It's important to note that behaviour can not be left out, as behaviour is the logical } \\
\text { next step after showing the virtual environment to users or within the design team. }\end{array}$ \\
\hline C3.3 Issues & 1. & $\begin{array}{l}\text { P4 indicates that one of the things he noticed is that you need to be able to } \\
\text { recognize tags from a larger distance, to include more context. FAC agrees that } \\
\text { technically this could indeed be done, and improve the experience of the current } \\
\text { demo. }\end{array}$ \\
\hline C3.4 Usability & 2. & $\begin{array}{l}\text { It is argued that when this tool is used frequently, the scripting will become familiar } \\
\text { for the prototypers. } \\
\text { Furthermore it is expected that scripting will be the way to go, because designers } \\
\text { won't do the behaviour programming even with visual programming languages. P4 } \\
\text { agrees that it would indeed be a good first start to provide designers with a tool to } \\
\text { combine the models and an environment, so steps } 1 \text { and } 3 \text {. } \\
\text { If you would use visual logic bricks you would quickly run into limitations because of } \\
\text { the advanced skills of the prototypers. This relates to the aim of the application; if } \\
\text { you need visualisation only, the first and third step are sufficient. If you need more, } \\
\text { for an evaluation etc, you would include the more complex behaviour step, } \\
\text { implemented by a prototyper. } \\
\text { It is ielated to the chances of the tool being used eventually; it modeling behaviour is } \\
\text { simple enough, the complete tool would be more useful than a light-weight version } \\
\text { only supporting modeling and scene integration. } \\
\text { Supporting several applications makes the tool(chain) more likely to be used more } \\
\text { often. }\end{array}$ \\
\hline C3.5 Suggestions & 1. & $\begin{array}{l}\text { P1 says that if the behaviour is left out, so only models and environment, it would } \\
\text { already be a useful application, for instance for Contextual Inquiry. If this is } \\
\text { considered a starting point platform it could be extended to behaviour programming } \\
\text { to also support other (evaluation/test) tasks. In the first form it would mainly be an } \\
\text { internal communication tool. If you want feedback about specific aspects, then } \\
\text { behaviour is needed. } \\
\text { P1 says that if the behaviour is left out, so only models and environment, it would } \\
\text { already be a useful application, for instance for Contextual Inquiry. If this is } \\
\text { considered a starting point platform it could be extended to behaviour programming } \\
\text { to also support other (evaluation/test) tasks. In the first form it would mainly be an } \\
\text { internal communication tool. If you want feedback about specific aspects, then } \\
\text { behaviour is needed. } \\
\text { Making the first step by only looking at modeling and scene integration is considered } \\
\text { feasible and very useful. }\end{array}$ \\
\hline
\end{tabular}




\section{A.2 Tool evaluation}

In the tool evaluation session four participants (see table A.2) were asked to create a virtual environment using SweetHome3D. After the session the participants filled out the evaluation form included in appendix A.2.1. The results of this evaluation are included in appendix A.2.2.

- All forms and answers have been translated from Dutch.

\begin{tabular}{lll}
\hline Participant & Label & Function \\
\hline P1 & UE & Usability Engineer \\
P2 & PD & Product Designer \\
P3 & UE & Usability Engineer \\
P4 & ID & Interaction Designer \\
\hline
\end{tabular}

Table A.2 List of participants and their labels.

\section{A.2.1 Tool evaluation form}

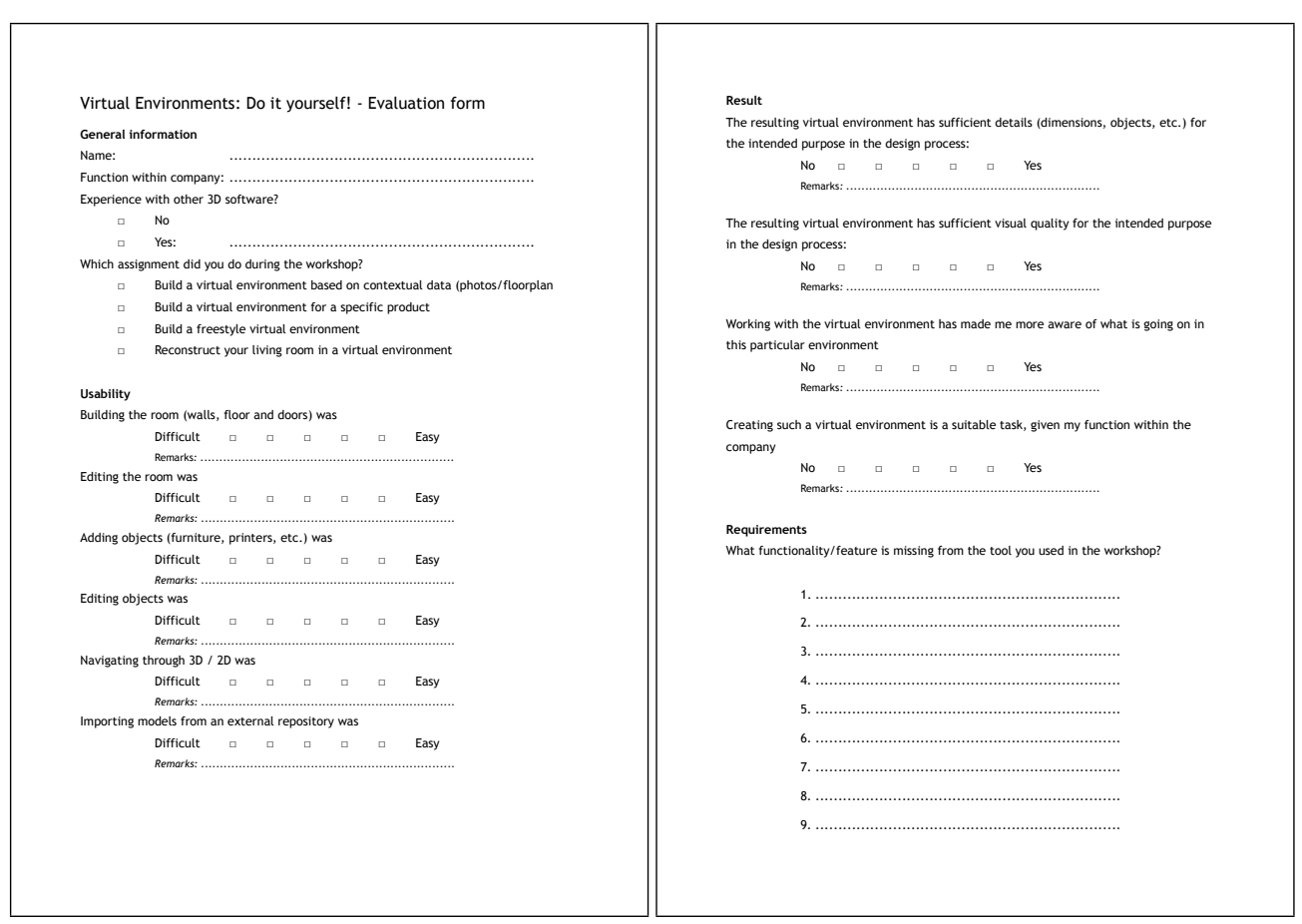




\section{A.2.2 Tool evaluation results}

\begin{tabular}{|c|c|c|c|c|}
\hline Experience with 3D & No & $\begin{array}{l}\text { Yes, currently Creo } \\
\text { Elements / proEngineer }\end{array}$ & No & No \\
\hline Assignment & $\begin{array}{l}\text { Build a virtual } \\
\text { environment based on } \\
\text { contextual data } \\
\text { (photos/floorplan) }\end{array}$ & $\begin{array}{l}\text { Build a virtual } \\
\text { environment for a } \\
\text { specific product }\end{array}$ & $\begin{array}{l}\text { Build a freestyle virtual } \\
\text { environment }\end{array}$ & $\begin{array}{l}\text { Reconstruct your living } \\
\text { room in a virtual } \\
\text { environment }\end{array}$ \\
\hline \multicolumn{5}{|c|}{ How difficult $(1-5,1=$ difficult $)$ are these sub tasks? } \\
\hline Building a room & 5 & 4 & 5 & 5 \\
\hline Editing a room & 5 & 3 & 4 & 5 \\
\hline Importing models & 3 & 4 & 3 & 5 \\
\hline \multicolumn{5}{|c|}{ How would you rate the resulting virtual environment? (1 = negative) } \\
\hline Sufficient details & 5 & 4 & 5 & 4 \\
\hline Visual quality & 5 & 4 & 4 & 4 \\
\hline Improves awareness & 4 & 4 & 4 & 4 \\
\hline Suitable task & 4 & 5 & 4 & 2 \\
\hline
\end{tabular}

The user interface of SweetHome3D (using a 2D and a 3D view) was appreciated by the participants. Especially the 2D floorplan combined with the list of 3D objects is very easy to use; it is a matter of dragging and dropping objects.

- For certain objects it would be useful to be able to edit specific parts (e.g. change the jacket colour of a user object, or one

face of a cabinet object). The current implementation only allows for changing the entire object.

- Object Library

For some of the assignments, more specific models were required in order to match the DSI information or specific product environments. One of the forms mentions the inclusion of a company specific object library, containing specific printers and other objects.

While the list of 3D objects is useful, it takes some time to find the object they were looking for. The categories are not specific enough to quickly find objects. For instance, objects like plants and waste bins were difficult to find, even when recalling it from memory.

After importing new models and objects, it is unclear where in the object list they are placed; there is no indication of new or old objects.

- Navigation \& Object Manipulation

- There are some inconsistencies between the 2D and 3D navigation windows. For instance, panning works as expected in $2 \mathrm{D}$ view, but it is not supported in $3 \mathrm{D}$ view. The virtual walk-through option does allow for view panning, but the directions are inverted.

For one participant, working on a particularly large environment $(70 \mathrm{~m}+)$ the 3D navigation failed, probably because it was not made for such environments.

- Using the mouse in the 2D view to edit objects was difficult, because the four control handle icons were often misinterpreted as buttons. While the icons represent the function, the user is actually required to click the object corner itself, not the icon. This often lead to users clicking the underlying floor instead of an object.

- When editing the 2D floorplan it would be useful to be able to group certain objects, so that when an object changes, its entire group is automatically updated. For instance, a table, chairs and desktop computers could be grouped into one office island.

- Participants indicate a need to quickly create simple 3D objects themselves, instead of just dragging predefined objects into the environment

- The 'people objects' currently included are quite limited; only static, standing people are available while 'sitting down' people are also required. People should also be editable, e.g. to change the color of their clothes.

- The virtual environments look a bit empty, because there are no 'random small office materials' available in the current object collection. Participants indicated that in such environments it would be nice to also make it look like it is actually being used. 


\section{A.3 Cross-company evaluation 1}

In the first cross-company evaluation the Virtual Printshop application, originally developed for company $\mathrm{A}$, was demonstrated to and evaluated by companies $\mathrm{B}$ and $\mathrm{C}$. After presenting and experiencing the application prototypes, the participants filled out the evaluation forms included in appendix A.3.1. The results of this evaluation are included in appendix A.3.2.

- All forms and answers have been translated from Dutch. 


\section{A.3.1 CCE1 forms}

REPAR-B // Cross-Company Evaluation 1

Company / Companies:

Group members:

This form contains questions that help you 'translate' the Virtual Printshop into something

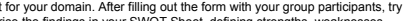
opportunities and threats for this VR application in your company.

The form consists of three parts:

Part $1 / /$ Creating the Virtual Environmen

Part $2 / /$ The Virtual Environment itself The order in which you fill out the form is not important, but please make sure you answer
all the questions.
Part $1 / /$ Creating The Virtual EnVIRonment As shown in the case study presentation, creating the virtual environment consists of three

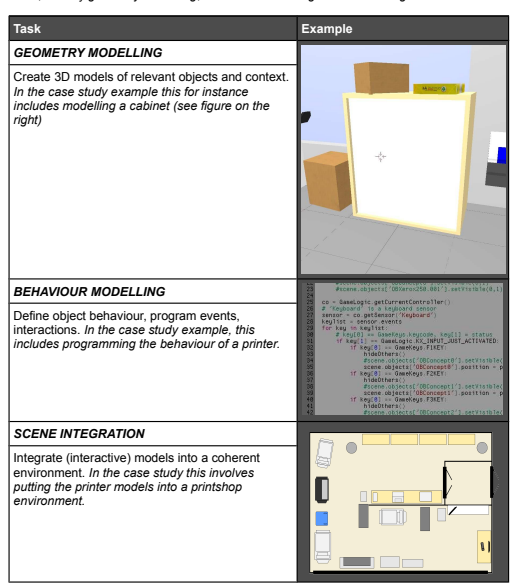

Allocation of Tools \& Skills

For each of the three tasks, please indicate if your company has the appropriate skills and tools avaliable for these tasks. If so, please indicale what lools you would consider appropriate (e.g. existing CAD tools or progr
what kind of skills and tools you would need.

\begin{tabular}{|c|c|c|}
\hline \multirow{2}{*}{$\begin{array}{ll}\text { Task } \\
1.1 \quad \text { GEOMETRY MODELLING } \\
\end{array}$} & \multirow[t]{2}{*}{ Tools available? } & \multirow[t]{2}{*}{ Skills available? } \\
\hline & & \\
\hline $\begin{array}{l}\text { Geometry modelling; creating 3D } \\
\text { models of your products }\end{array}$ & 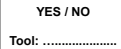 & $\begin{array}{l}\text { YES / NO } \\
\text { Who: ................... }\end{array}$ \\
\hline $\begin{array}{l}\text { If tools are not available, would } \\
\text { the tools shown in the case } \\
\text { study be suitable for you? }\end{array}$ & & \\
\hline $\begin{array}{l}\text { - If skills are not available, how } \\
\text { would you solve this? }\end{array}$ & & \\
\hline 1.2 BEHAVIOUR MODELLING & & \\
\hline $\begin{array}{l}\text { Behaviour modelling; adding behaviour } \\
\text { to objects, creating virtual scenarios, } \\
\text { programming events, etc. }\end{array}$ & $\begin{array}{c}\text { YES / NO } \\
\text { Tool: }: . . . . . . \cdots \cdots \cdots . . . . . \\
\end{array}$ & $\begin{array}{c}\text { YES / NO } \\
\text { Who: } \ldots \ldots \ldots \ldots \ldots \ldots \ldots . . .\end{array}$ \\
\hline $\begin{array}{l}\text { - If tools are not available, would } \\
\text { the tools shown in the case } \\
\text { study be suitable for you? }\end{array}$ & & \\
\hline $\begin{array}{l}\text { If skills are not available, how } \\
\text { would you solve this? }\end{array}$ & & \\
\hline 1.3 SCENE INTEGRATION & & \\
\hline $\begin{array}{l}\text { Scene integration; integrating } 3 D \\
\text { models into a virtual room/scene }\end{array}$ & $\begin{array}{c}\text { YES / NO } \\
\text { Tool: } \ldots \ldots \ldots \ldots \ldots \ldots\end{array}$ & 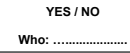 \\
\hline $\begin{array}{l}\text { - If tools are not available, would } \\
\text { the tools shown in the case } \\
\text { study be suitable for you? }\end{array}$ & & \\
\hline $\begin{array}{l}\text { - If skills are not available, how } \\
\text { would you solve this? }\end{array}$ & & \\
\hline
\end{tabular}

PART $2 / /$ THE VIRTUAL ENVIRONMENT

The virtual environment consists of a virtual context (e.g. a room, an outside space, a factory hall, etc.) and virtual objects (e.g. your products, furniture, people, etc.). Everything in the virtual environment must somehow be created.

The questions in part 2 are about the virtual context and the virtual objects. The image
below briefly explains the difference between these two to help you answer the questions.

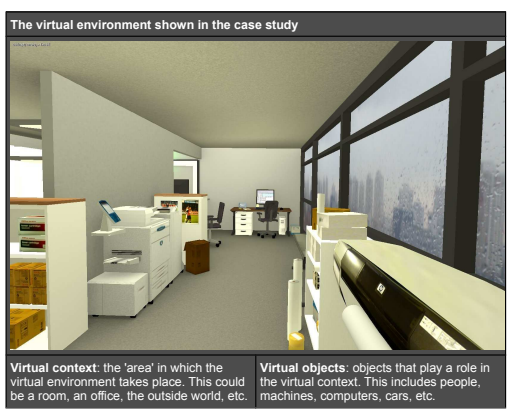




\section{CCE1 forms (continued)}

Describe the Virtual Context

2.1 Our virtual context would consist of

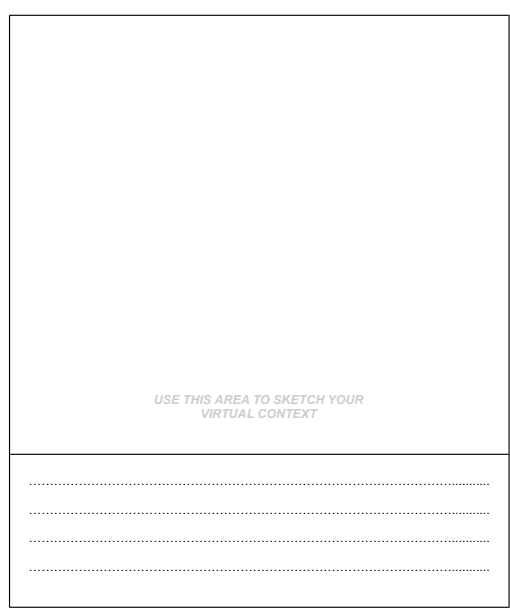

Describe the Virtual Objects

2.5 Which virtual objects would you include in the virtual environments (in order of importance)? Virtual objects are things like people, utilities, computers, machines,
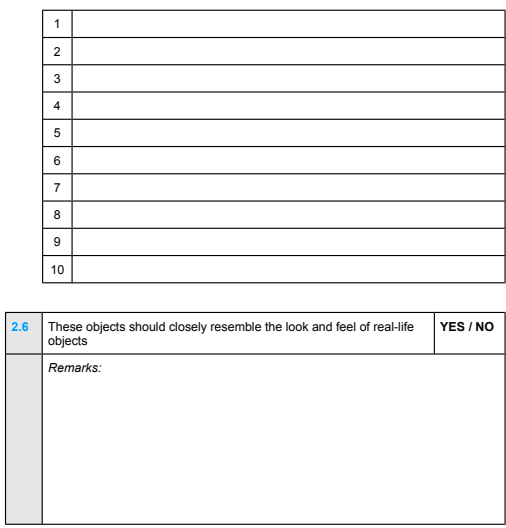

\begin{tabular}{|l|l|l}
\hline 2.2 & This context is roughly the same for each test/study we do & YES / NO \\
\hline & Regs
\end{tabular}

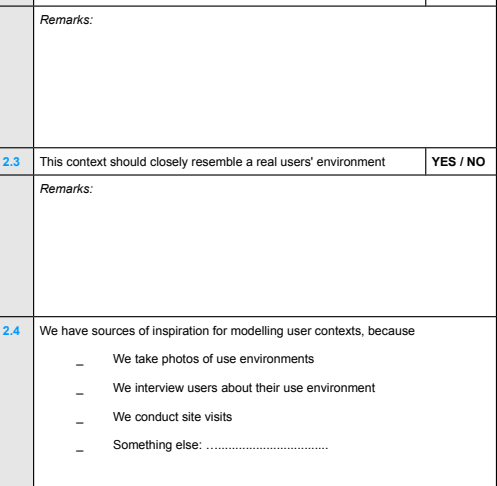

Compare the VE to the Case Study

Based on what you have seen from the presented case study, how would your virtual would it be sufficient for you to reduce the interactivity, realism or complexity?

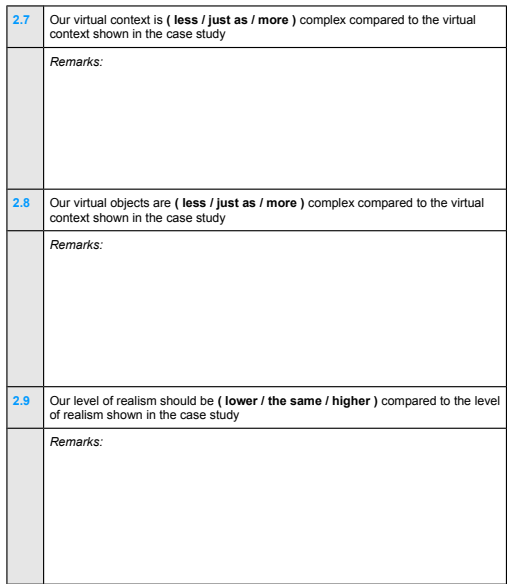




\section{CCE1 forms (continued)}

PART 3 // USING THE ViRTUAL ENVIRONMENT

Imagine you created the virtual environment according to your requirements, how would you use it in practice, and how would it help you? These questions are about actually
using the virtual environment in a user study. What would be the main purpose of this

\begin{tabular}{|c|c|}
\hline \multirow[t]{7}{*}{3.1} & Our test subjects/participants would be (multiple answers allowed) \\
\hline & _. End-users \\
\hline & _ End-user representatives \\
\hline & _ $\quad$ Decision makers \\
\hline & _ Us (designers, engineers) \\
\hline & Other departments within the company \\
\hline & _ $\quad$ Something else: ............................... \\
\hline \multirow[t]{6}{*}{3.2} & The teststudy would primarily be for (one answer) \\
\hline & - Idea generation \\
\hline & - Concept evaluation \\
\hline & $\circ \quad$ Detailed analysis \\
\hline & - Exact simulation and measurements \\
\hline & Something else: .......................... \\
\hline \multirow[t]{6}{*}{3.3} & The test/study would also be useful for (multiple answers allowed) \\
\hline & _ Idea generation \\
\hline & _ Concept evaluation \\
\hline & _ Detailed analysis \\
\hline & _. Exact simulation and measurements \\
\hline & 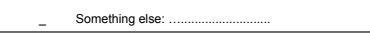 \\
\hline
\end{tabular}

NeXt // Present your findings

There are two Powerpoint sheets for you to present your findings.

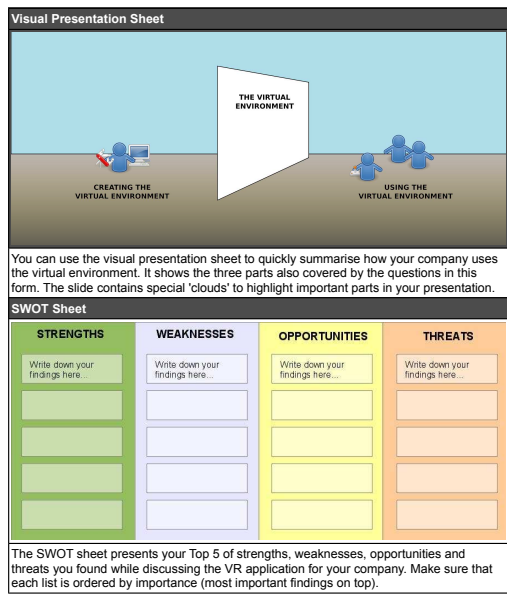

\begin{tabular}{|c|c|}
\hline 3.4 & \multirow{2}{*}{\begin{tabular}{|l} 
The study would be designed by ..................... (a name or job description). \\
The most important benefits of using this virtual environment in user studies are \\
(number in order of importance)
\end{tabular}} \\
\hline \multirow[t]{7}{*}{3.5} & \\
\hline & Detecting design errors in an early stage \\
\hline & ... Improving the involvement of end-users \\
\hline & ... Time reduction of the conceptual design stage \\
\hline & $\ldots \quad$ Something else: ………............................ \\
\hline & Something else: ............ \\
\hline & $\ldots \quad$... Something else: ……… \\
\hline 3.6 & $\begin{array}{l}\text { A user study involving the virtual environment would } \\
\text { take place about }<\ldots \text {.... times a year. }\end{array}$ \\
\hline
\end{tabular}




\section{A.3.2 CCE1 results}

\begin{tabular}{|c|c|c|}
\hline Part 1 & Company B & Company C \\
\hline \multicolumn{3}{|l|}{ 1.1 Geometry modelling } \\
\hline $\begin{array}{l}\text { Are Geometry modelling } \\
\text { tools and skills } \\
\text { available? If not, how } \\
\text { would you solve this? }\end{array}$ & Yes, tools and skills are available & Yes, tools and skills are available \\
\hline \multicolumn{3}{|l|}{ 1.2 Behaviour modelling } \\
\hline $\begin{array}{l}\text { Are Behaviour modelling } \\
\text { tools and skills } \\
\text { available? If not, how } \\
\text { would you solve this? }\end{array}$ & Yes, tools and skills are available & No, won't need/use it \\
\hline \multicolumn{3}{|l|}{ 1.3 Scene integration } \\
\hline $\begin{array}{l}\text { Are Scene integration } \\
\text { tools and skills } \\
\text { available? If not, how } \\
\text { would you solve this? }\end{array}$ & $\begin{array}{l}\text { "No, but Sweethome3d could be used for cabin } \\
\text { layout or storage layout of personal } \\
\text { belongings. We would learn this by self- } \\
\text { training or education" }\end{array}$ & $\begin{array}{l}\text { "Sweethome3d is applicable here. We would } \\
\text { learn this by training" }\end{array}$ \\
\hline
\end{tabular}

2.1 The virtual context would consist of...

Roads and traffic situations for driving studies, Factory layout visualisation, with walls, cabin for non-driving studies collumns, ceiling, routings of raw materia and products, our equipment, storage of tools, cleaning room, Third party equipment; mixing area, cable tracking

2.2 This context is roughly the same for each test/study we do

No; different road types (city, urban, highway), different traffic conditions (behaviour of other cars), different environments (hills, dark, rain)

Yes; usually the same environment; a factory context with our machines in it

2.3 This context should closely resemble a real user's environment

\begin{tabular}{|l|l|l|l|}
\hline & \multicolumn{1}{|l|}{ Yes } & \multicolumn{1}{l|}{$\begin{array}{l}\text { Yes, a realistic representation should give a } \\
\text { better experience, and with that more reliable } \\
\text { feedback }\end{array}$} \\
\hline 2.4 We have sources of inspiration for modelling user contexts, because \\
\hline & $\begin{array}{l}\text { We take photos of use environments } \\
\text { We interview users about their use } \\
\text { environment } \\
\text { We conduct site visits } \\
\text { We drive trucks ourselves }\end{array}$ & $\begin{array}{l}\text { - We take photos of use environments } \\
\text { We conduct site visits } \\
\text { We use technical drawings }\end{array}$ \\
\hline & $\begin{array}{l}\text { Truck behaviour, other road users, wheather } \\
\text { conditions, road conditions, traffic scenarios }\end{array}$ & $\begin{array}{l}\text { Company equipment, 3rd party equipment, } \\
\text { walls, collumns, ceiling, routing of materials } \\
\text { and goods, tool storage }\end{array}$ \\
\hline
\end{tabular}




\section{CCE1 results (continued)}

2.6 These objects should closely resemble the look and feel of real-life objects Yes; To validate safety aspects, to have context validity

Yes; The look and feel of the machines should be realistic, the context is less important (floors, walls)

2.7 Our virtual context is ( less / just as / more ) complex compared to the virtual context shown in the case study

\begin{tabular}{|l|l|l|}
\hline \multicolumn{2}{|l|}{ More } & \multicolumn{1}{l|}{ Just as } \\
\hline 2.8 Our virtual objects are (less / just as / more ) complex compared to the virtual context shown in the case study \\
\hline & More; Especially behaviour of other road users & Just as \\
\hline 2.9 Our level of realism should be (lower / the same / higher) compared to the level of realism shown in the case study \\
\hline & $\begin{array}{l}\text { The same; Behaviour is same or more? } \\
\text { Looks/aestetics is less }\end{array}$ & $\begin{array}{l}\text { The same; It would be interesting to include } \\
\text { sound in the experience. }\end{array}$ \\
\hline
\end{tabular}

\begin{tabular}{|c|c|c|}
\hline Part 3 & Company B & Company C \\
\hline \multicolumn{3}{|c|}{ 3.1 Our test subjects/participants would be (multiple answers allowed) } \\
\hline & $\begin{array}{ll}\text { - } & \text { End-user representatives, } \\
\text { - } & \text { Decision makers, and } \\
& \text { Us (designers, engineers) }\end{array}$ & $\begin{array}{ll}\text { - } & \text { End-users, } \\
\text { - } & \text { End-user representatives, } \\
\text { - } & \text { Decision makers, } \\
\text { - Us (designers, engineers), } \\
\text { : } & \text { Other departments in the company, } \\
\text { - Sales department }\end{array}$ \\
\hline \multicolumn{3}{|c|}{ 3.2 The test/study would primarily be for (one answer) } \\
\hline & Concept evaluation & Concept evaluation \\
\hline \multicolumn{3}{|c|}{ 3.3 The test/study would also be useful for (multiple answers allowed) } \\
\hline & $\begin{array}{l}\text { Detailed analysis } \\
\text { Exact simulation and measurements }\end{array}$ & Visualisation \\
\hline \multicolumn{3}{|c|}{ 3.4 The study would be designed by ........................... (a name or job description). } \\
\hline & Vehicle testing and vehicle definition & Product development \\
\hline \multicolumn{3}{|c|}{ 3.5 The most important benefits of using this virtual environment in user studies are (number in order of importance) } \\
\hline & $\begin{array}{l}\text { 1. Time reduction of the conceptual design } \\
\text { stage } \\
\text { 2. Detecting errors in an early stage } \\
\text { 3. Reduce number/costs of physical } \\
\text { prototypes } \\
\text { 4. Many virtual iterations and then 1st time } \\
\text { 5. Decision making (management review) } \\
\text { 6. Improving involvement of end-users }\end{array}$ & $\begin{array}{l}\text { 1. Avoiding mis-communication } \\
\text { 2. Detecting design errors in an early stage } \\
\text { 3. Improving the involvement of end-users } \\
\text { 4. Time reduction of the conceptual design } \\
\text { stage }\end{array}$ \\
\hline \multicolumn{3}{|c|}{ 3.6 A user study involving the virtual environment would take place about $<\ldots \ldots . .>$ times a year. } \\
\hline & 4 & 5 \\
\hline
\end{tabular}


B $\quad$ Case Study 2 Data 


\section{B.1 Application validation}

The application validation session of case study 2 involved two groups of four participants, as listed in table B.1. During the session, the participants carried out a design session with the Virtual Persona application. After the session they were given the evaluation form included in appendix B.1.1. The results of this evaluation are included in appendix B.1.2.

- All forms and answers have been translated from Dutch.

\begin{tabular}{lll}
\hline Group & Participant & Function \\
\hline 1 & P1 & CAD/Prototyping \\
& P2 & HMI group \\
& P3 & HMI group \\
& P4 & Vehicle definition \\
\hline 2 & P1 & Styling \\
& P2 & HMI group \\
& P3 & Cabin group \\
& P4 & HMI group \\
\hline
\end{tabular}

Table B.1 List of participants and their labels. 


\section{B.1.1 Application validation form}

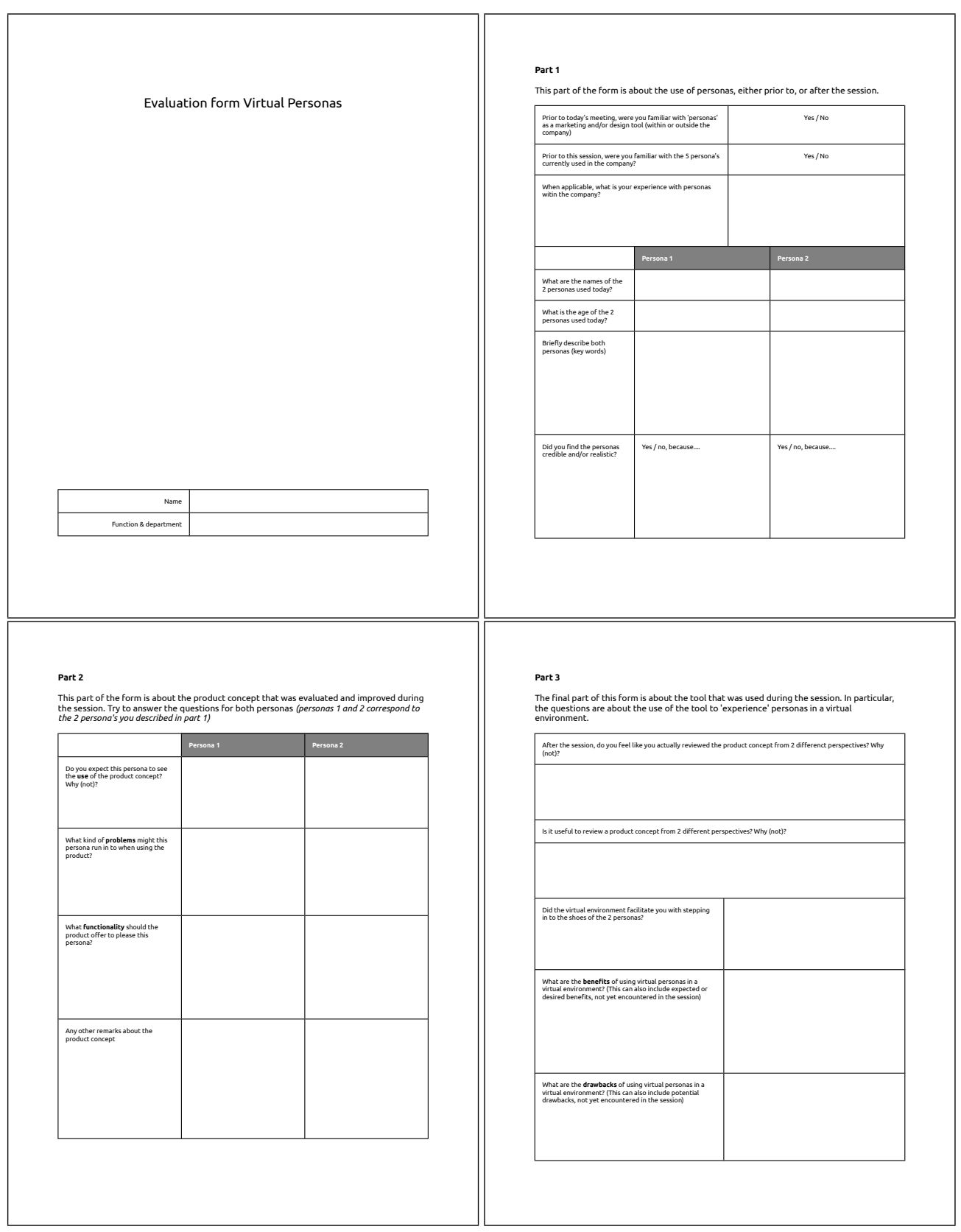




\section{B.1.2 Application validation results}

\begin{tabular}{|c|c|c|c|c|c|c|c|c|}
\hline Part 1 & \multicolumn{2}{|l|}{ G1.P1 } & \multicolumn{2}{|l|}{ G1.P2 } & \multicolumn{2}{|l|}{ G1.P3 } & \multicolumn{2}{|l|}{ G1.P4 } \\
\hline $\begin{array}{l}1.1 \text { Are you familiar with } \\
\text { 'personas' as a } \\
\text { marketing or design tool } \\
\text { (in or outside the } \\
\text { company) }\end{array}$ & \multicolumn{2}{|l|}{ Yes } & \multicolumn{2}{|l|}{ Yes } & \multicolumn{2}{|l|}{ Yes } & \multicolumn{2}{|l|}{ Yes } \\
\hline $\begin{array}{l}1.2 \text { Are you familiar with } \\
\text { the company's } 5 \\
\text { personas? }\end{array}$ & \multicolumn{2}{|l|}{ No } & \multicolumn{2}{|l|}{ Yes } & \multicolumn{2}{|l|}{ Yes } & \multicolumn{2}{|l|}{ Yes } \\
\hline $\begin{array}{l}1.3 \text { What is your } \\
\text { experiences (if any) with } \\
\text { personas? }\end{array}$ & & & \multicolumn{2}{|l|}{ None } & \multicolumn{2}{|l|}{ None } & \multicolumn{2}{|l|}{ Very limited } \\
\hline $\begin{array}{l}1.4 \text { What are the names } \\
\text { of the } 2 \text { personas used } \\
\text { in the session? }\end{array}$ & Jim & Stanley & Jim & Stanley & Jim & Stanley & Jim & Stanley \\
\hline $\begin{array}{l}1.7 \text { Do you consider the } \\
\text { personas to be realistic? }\end{array}$ & Yes & Yes & Yes & Yes & $\begin{array}{l}\text { Yes, thanks to } \\
\text { the introduction } \\
\text { movies and } \\
\text { visual support }\end{array}$ & $\begin{array}{l}\text { Yes, thanks to } \\
\text { the introduction } \\
\text { movies and } \\
\text { visual support }\end{array}$ & $\begin{array}{l}\text { Yes, typical } \\
\text { younger } \\
\text { generation }\end{array}$ & $\begin{array}{l}\text { Yes, old } \\
\text { generation, } \\
\text { east-european } \\
\text { truckers }\end{array}$ \\
\hline
\end{tabular}

\begin{tabular}{|c|c|c|c|c|c|c|c|c|}
\hline Part 1 & \multicolumn{2}{|l|}{ G2.P1 } & \multicolumn{2}{|l|}{ G2.P2 } & \multicolumn{2}{|l|}{ G2.P3 } & \multicolumn{2}{|l|}{ G2.P4 } \\
\hline \multicolumn{9}{|l|}{ The use of personas } \\
\hline $\begin{array}{l}1.1 \text { Are you familiar with } \\
\text { 'personas' as a } \\
\text { marketing or design tool } \\
\text { (in or outside the } \\
\text { company) }\end{array}$ & \multicolumn{2}{|l|}{ Yes } & \multicolumn{2}{|l|}{ Yes } & \multicolumn{2}{|l|}{ Yes } & \multicolumn{2}{|l|}{ Yes } \\
\hline $\begin{array}{l}1.2 \text { Are you familiar with } \\
\text { the company's } 5 \\
\text { personas? }\end{array}$ & \multicolumn{2}{|l|}{ Yes } & \multicolumn{2}{|l|}{ No } & \multicolumn{2}{|l|}{ Yes } & \multicolumn{2}{|l|}{ Yes } \\
\hline $\begin{array}{l}1.3 \text { What is your } \\
\text { experiences (if any) with } \\
\text { personas? }\end{array}$ & \multicolumn{2}{|c|}{$\begin{array}{l}\text { Very useful method; everyone is } \\
\text { different/special, personas are } \\
\text { good to find a middle way in design }\end{array}$} & \multicolumn{2}{|l|}{ None } & \multicolumn{2}{|c|}{$\begin{array}{l}\text { Only used during graduation } \\
\text { assignment }\end{array}$} & \multicolumn{2}{|c|}{$\begin{array}{l}\text { Very rare, only once during } \\
\text { graduation }\end{array}$} \\
\hline $\begin{array}{l}1.4 \text { What are the names } \\
\text { of the } 2 \text { personas used } \\
\text { in the session? }\end{array}$ & Jim & Stanley & Jim & Stanley & Jim & Stanley & Jim & Stanley \\
\hline $\begin{array}{l}1.7 \text { Do you consider the } \\
\text { personas to be realistic? }\end{array}$ & Yes & Yes & Yes & Yes & $\begin{array}{l}\text { Yes, represents } \\
\text { young/new } \\
\text { generation of } \\
\text { truckers. Looks } \\
\text { realistic to me }\end{array}$ & $\begin{array}{l}\text { Yes, same as } \\
\text { Jim, but for the } \\
\text { older } \\
\text { generation }\end{array}$ & $\begin{array}{l}\text { Yes, I know this } \\
\text { type of trucker } \\
\text { exists }\end{array}$ & $\begin{array}{l}\text { Yes, I know this } \\
\text { type of trucker } \\
\text { exists }\end{array}$ \\
\hline
\end{tabular}




\section{Application validation results (continued)}

\begin{tabular}{|c|c|c|c|c|c|c|c|c|}
\hline Part 2 & \multicolumn{2}{|l|}{ G1.P1 } & \multicolumn{2}{|l|}{ G1.P2 } & \multicolumn{2}{|l|}{ G1.P3 } & \multicolumn{2}{|l|}{ G1.P4 } \\
\hline \multicolumn{9}{|l|}{ About the product concept } \\
\hline $\begin{array}{l}2.1 \text { Do you expect this } \\
\text { user to see the use of } \\
\text { the product concept? } \\
\text { Why (not)? }\end{array}$ & Yes, he likes it & No & $\begin{array}{l}\text { Yes, he'll look } \\
\text { into it and likes } \\
\text { to have as much } \\
\text { functions/featur } \\
\text { es as possible }\end{array}$ & $\begin{array}{l}\text { No, not } \\
\text { interested at all }\end{array}$ & $\begin{array}{l}\text { Yes, a lot of } \\
\text { options and it's } \\
\text { a great toy }\end{array}$ & $\begin{array}{l}\text { No, doesn't like } \\
\text { using a } \\
\text { smartphone }\end{array}$ & $\begin{array}{l}\text { Yes, cool } \\
\text { features, added } \\
\text { functionality }\end{array}$ & $\begin{array}{l}\text { No, doesn't } \\
\text { need these } \\
\text { functions }\end{array}$ \\
\hline $\begin{array}{l}2.2 \text { Which problems do } \\
\text { you expect the user to } \\
\text { encounter with the } \\
\text { product concept? }\end{array}$ & $\begin{array}{l}\text { Smartphone } \\
\text { mallfunction }\end{array}$ & & $\begin{array}{l}\text { Used a lot so } \\
\text { drains battery, } \\
\text { missing just that } \\
\text { specific extra } \\
\text { function }\end{array}$ & $\begin{array}{l}\text { Accidentally } \\
\text { activating/doing } \\
\text { something with } \\
\text { the remote and } \\
\text { not knowing } \\
\text { how to solve it }\end{array}$ & $\begin{array}{l}\text { He may forget } \\
\text { the traditional } \\
\text { controls (e.g. } \\
\text { taking key) }\end{array}$ & $\begin{array}{l}\text { He can't use all } \\
\text { options/feature } \\
\text { s, but doesn't } \\
\text { mind it }\end{array}$ & $\begin{array}{l}\text { No backup after } \\
\text { malfunctions or } \\
\text { power outage }\end{array}$ & $\begin{array}{l}\text { He'll miss out on } \\
\text { functions and } \\
\text { comfort/control }\end{array}$ \\
\hline $\begin{array}{l}2.3 \text { Which product } \\
\text { functionality is } \\
\text { important for this user? }\end{array}$ & $\begin{array}{l}\text { Extra } \\
\text { functionality }\end{array}$ & & $\begin{array}{l}\text { Makes job } \\
\text { interesting, } \\
\text { mood } \\
\text { corrections } \\
\text { (light) }\end{array}$ & $\begin{array}{l}\text { None, apart } \\
\text { from business } \\
\text { functions }\end{array}$ & $\begin{array}{l}\text { As much } \\
\text { options/feature } \\
\text { s as possible, a } \\
\text { lot of } \\
\text { configurability }\end{array}$ & $\begin{array}{l}\text { Doesn't matter, } \\
\text { he won't use it } \\
\text { anyway }\end{array}$ & $\begin{array}{l}\text { Backup } \\
\text { function, added } \\
\text { features }\end{array}$ & Simplicity \\
\hline
\end{tabular}

\begin{tabular}{|c|c|c|c|c|c|c|c|c|}
\hline Part 2 & \multicolumn{2}{|l|}{ G2.P1 } & \multicolumn{2}{|l|}{ G2.P2 } & \multicolumn{2}{|l|}{ G2.P3 } & \multicolumn{2}{|l|}{ G2.P4 } \\
\hline \multicolumn{9}{|l|}{ About the product concept } \\
\hline $\begin{array}{l}2.1 \text { Do you expect this } \\
\text { user to see the use of } \\
\text { the product concept? } \\
\text { Why (not)? }\end{array}$ & $\begin{array}{l}\text { Yes, this adds } \\
\text { something to } \\
\text { daily use }\end{array}$ & $\begin{array}{l}\text { Nee, only if it's } \\
\text { very accessible. } \\
\text { Doesn't see the } \\
\text { use of the } \\
\text { smartphone }\end{array}$ & $\begin{array}{l}\text { Yes, he will } \\
\text { investigate the } \\
\text { possibilities, and } \\
\text { use them when } \\
\text { possible }\end{array}$ & $\begin{array}{l}\text { No, he will } \\
\text { always rely on } \\
\text { the traditional } \\
\text { way of carrying } \\
\text { out tasks }\end{array}$ & $\begin{array}{l}\text { Yes, he's used to } \\
\text { using these } \\
\text { things }\end{array}$ & $\begin{array}{l}\text { Nee, oposite of } \\
\text { Jim }\end{array}$ & $\begin{array}{l}\text { Yes, he grew up } \\
\text { with technology } \\
\text { like this en is } \\
\text { very capable of } \\
\text { using them. }\end{array}$ & $\begin{array}{l}\text { Eventually yes, } \\
\text { because it will } \\
\text { make his job } \\
\text { easier. The } \\
\text { threshold is } \\
\text { high, and he will } \\
\text { only use a small } \\
\text { part of all } \\
\text { functions/featur } \\
\text { es }\end{array}$ \\
\hline $\begin{array}{l}2.2 \text { Which problems do } \\
\text { you expect the user to } \\
\text { encounter with the } \\
\text { product concept? }\end{array}$ & $\begin{array}{l}\text { Empty battery, } \\
\text { unfamiliar with } \\
\text { app }\end{array}$ & $\begin{array}{l}\text { It will take to } \\
\text { long to use the } \\
\text { smartphone app }\end{array}$ & $\begin{array}{l}\text { Not a lot of } \\
\text { issues as long as } \\
\text { the software is } \\
\text { intuitive }\end{array}$ & $\begin{array}{l}\text { Even with a } \\
\text { simple interface } \\
\text { he'll run into } \\
\text { problems }\end{array}$ & $\begin{array}{l}\text { Slow response } \\
\text { of system, } \\
\text { annoying for } \\
\text { him. Incomplete } \\
\text { or wrong data, } \\
\text { Malfunctions in } \\
\text { communication } \\
\text { between app } \\
\text { and truck }\end{array}$ & $\begin{array}{l}\text { Doesn't know } \\
\text { how to use the } \\
\text { app in case he's } \\
\text { forced to use it. } \\
\text { Takes a lot of } \\
\text { time, makes } \\
\text { errors }\end{array}$ & $\begin{array}{l}\text { No network } \\
\text { connection, } \\
\text { battery empty } \\
\text { because of } \\
\text { intense use }\end{array}$ & Learning curve \\
\hline $\begin{array}{l}2.4 \text { Other remarks about } \\
\text { the product concept? }\end{array}$ & & & & & $\begin{array}{l}\text { It can damage if } \\
\text { you drop it, it } \\
\text { can be stolen, it } \\
\text { can get dirty (oil } \\
\text { etc), can't use } \\
\text { the truck } \\
\text { functions if the } \\
\text { smartphone } \\
\text { fails }\end{array}$ & & $\begin{array}{l}\text { Distinguish } \\
\text { work from } \\
\text { private } \\
\text { functions, and } \\
\text { social media is } \\
\text { important for } \\
\text { Jim's }\end{array}$ & $\begin{array}{l}\text { Keep it simple, } \\
\text { business } \\
\text { oriented }\end{array}$ \\
\hline
\end{tabular}




\section{Application validation results (continued)}

\begin{tabular}{|c|c|c|c|c|}
\hline Part 3 & G1.P1 & G1.P2 & G1.P3 & G1.P4 \\
\hline $\begin{array}{l}3.1 \text { Do you have the idea } \\
\text { that you have been } \\
\text { evaluating the product } \\
\text { concept from two } \\
\text { different user } \\
\text { perspectives? }\end{array}$ & $\begin{array}{l}\text { Yes, you experience the concept } \\
\text { from different angles }\end{array}$ & $\begin{array}{l}\text { Yes, seeing the characters helps } \\
\text { keeping the group focused, less } \\
\text { chaotic brainstorms }\end{array}$ & $\begin{array}{l}\text { Yes, primarily difference between } \\
\text { tech/non-tech savy }\end{array}$ & $\begin{array}{l}\text { Yes, maybe a female persona } \\
\text { would also be interesting }\end{array}$ \\
\hline $\begin{array}{l}3.3 \text { Did the virtual } \\
\text { environment help you to } \\
\text { step into the shoes of } \\
\text { the two personas? }\end{array}$ & Yes & Yes & Yes, it sets the mood in the session & $\begin{array}{l}\text { Yes. It could also help to improve } \\
\text { the introduction of the 'lifestyle' of } \\
\text { each persona }\end{array}$ \\
\hline $\begin{array}{l}3.5 \text { What are the } \\
\text { drawbacks or limitations } \\
\text { of using the virtual } \\
\text { environment in this } \\
\text { session? }\end{array}$ & Takes time to extend & Takes time, and it may distract & $\begin{array}{l}\text { Could be negative; you might } \\
\text { forget things if the environment } \\
\text { doesn't show them }\end{array}$ & $\begin{array}{l}\text { Maybe we focussed to much on the } \\
\text { 'perfect user' }\end{array}$ \\
\hline
\end{tabular}

\begin{tabular}{|c|c|c|c|c|}
\hline Part 3 & G2.P1 & G2.P2 & G2.P3 & G2.P4 \\
\hline $\begin{array}{l}3.1 \text { Do you have the idea } \\
\text { that you have been } \\
\text { evaluating the product } \\
\text { concept from two } \\
\text { different user } \\
\text { perspectives? }\end{array}$ & $\begin{array}{l}\text { Yes, it provides very different use } \\
\text { perspectives }\end{array}$ & $\begin{array}{l}\text { Yes, but the interface is limited. } \\
\text { Things go automatically that } \\
\text { should be manual. It does help with } \\
\text { stepping into a particular scenario }\end{array}$ & $\begin{array}{l}\text { We did not focus on the } \\
\text { smartphone that much }\end{array}$ & $\begin{array}{l}\text { No, the smartphone concept is not } \\
\text { the focus of the session. The person } \\
\text { is holding something, but that's it. } \\
\text { We should be able to zoom in on } \\
\text { the smartphone, and edit the UI on } \\
\text { the fly. Alternatively the UI could } \\
\text { be shown in a seperate window all } \\
\text { the time. }\end{array}$ \\
\hline $\begin{array}{l}3.2 \text { Do you think it is } \\
\text { useful to evaluate } \\
\text { product concepts from } \\
\text { different user } \\
\text { perspectives? If so, why } \\
\text { (not)? }\end{array}$ & $\begin{array}{l}\text { Yes, it's a good way to determine } \\
\text { target groups and find out what } \\
\text { this target group means to the } \\
\text { product }\end{array}$ & $\begin{array}{l}\text { Yes, it definitely has added value in } \\
\text { the early (brainstorm) phases of a } \\
\text { project. In advanced stages a more } \\
\text { detailed tool is needed. }\end{array}$ & $\begin{array}{l}\text { I think so, otherwise you might not } \\
\text { notice certain specific things }\end{array}$ & $\begin{array}{l}\text { Yes, everyone is different from } \\
\text { each other, and everyone has } \\
\text { particular needs }\end{array}$ \\
\hline $\begin{array}{l}3.3 \text { Did the virtual } \\
\text { environment help you to } \\
\text { step into the shoes of } \\
\text { the two personas? }\end{array}$ & Yes, very much & Yes & $\begin{array}{l}\text { Yes, you can design something for a } \\
\text { specific target group }\end{array}$ & $\begin{array}{l}\text { Yes, the introduction movies and all } \\
\text { the elements in it are valuable }\end{array}$ \\
\hline $\begin{array}{l}3.5 \text { What are the } \\
\text { drawbacks or limitations } \\
\text { of using the virtual } \\
\text { environment in this } \\
\text { session? }\end{array}$ & $\begin{array}{l}\text { Interactions, and to remind you of } \\
\text { simple things (such as opening } \\
\text { doors) }\end{array}$ & $\begin{array}{l}\text { Real circumstances are missing, } \\
\text { such as time pressure. These make } \\
\text { the experience slightly less realistic }\end{array}$ & $\begin{array}{l}\text { It's timetaking/cumbersome to } \\
\text { work with the scenario, difficult to } \\
\text { get the right level of detail }\end{array}$ & $\begin{array}{l}\text { You can't do everything in the } \\
\text { environment, it still takes some } \\
\text { imagination }\end{array}$ \\
\hline
\end{tabular}




\section{B.2 Tool selection}

The tool selection session involved five participants (see table B.2) who were asked to discuss how to implement the Virtual Persona application in the company's existing product development process. The forms included in appendix B.2.1 were used to initiate the discussion. Participants discussed each part of the virtual environment (i.e. the truck, the persona's, the world and the topic of interest) in terms of use frequency, required resources, task allocation and criteria. The results of this discussion are included in appendix B.2.2.

- All forms and answers have been translated from Dutch.

\begin{tabular}{ll}
\hline Participant & Function \\
\hline P1 & Cabin/Ergonomics group \\
P2 & Product planning \\
P3 & Testing department \\
P4 & HMl expert \\
P5 & Vehicle definition \\
\hline
\end{tabular}

Table B.2 List of participants and their labels. 


\section{B.2.1 Tool selection forms}
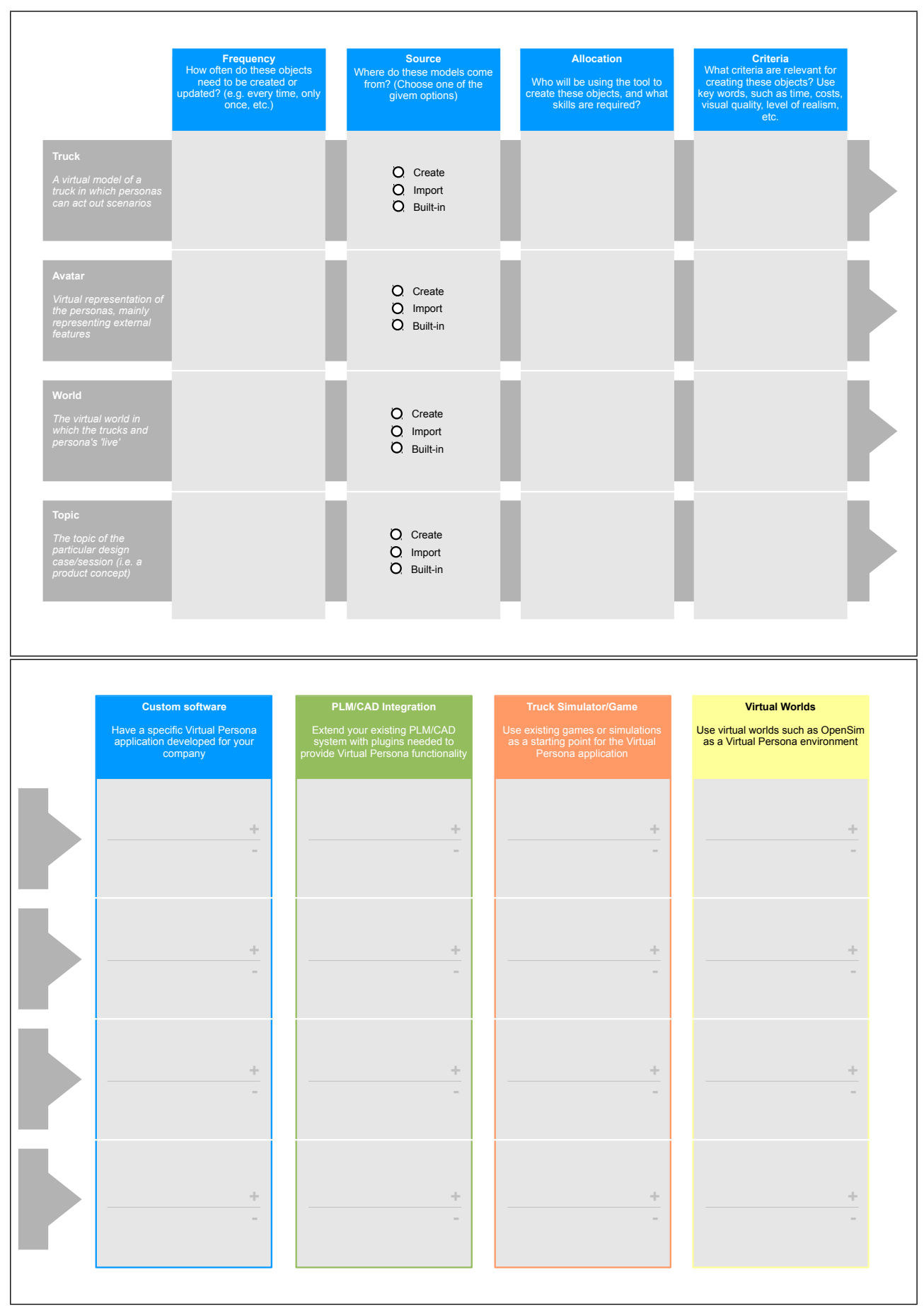


\section{B.2.2 Tool selection results}

\begin{tabular}{|l|l|l|l|l|l|}
\hline P & Object & Frequency & Source & Allocation & Criteria \\
\hline 1 & Truck & $\begin{array}{l}\text { Each session. } \\
\text { Environment doesn't } \\
\text { change that much, the } \\
\text { topic itself changes } \\
\text { each session }\end{array}$ & $\begin{array}{l}\text { Create } \\
\text { Import }\end{array}$ & $\begin{array}{l}\text { Cabin department, } \\
\text { CAD engineers }\end{array}$ & $\begin{array}{l}\text { You need to have } \\
\text { some knowledge of } \\
\text { the design/topic }\end{array}$ \\
\cline { 2 - 6 } & Avatar & Limited / low & Built-in & $\begin{array}{l}\text { Vehicle definition } \\
\text { creates the avatars, } \\
\text { specialists integrate it } \\
\text { in the environment }\end{array}$ & $\begin{array}{l}\text { Knowledge about } \\
\text { drivers and situations }\end{array}$ \\
\cline { 2 - 7 } & World & $\begin{array}{l}\text { Limited / low } \\
\text { Vehicle definition } \\
\text { creates the avatars, } \\
\text { specialists integrate it } \\
\text { in the environment }\end{array}$ & $\begin{array}{l}\text { You need to know the } \\
\text { environment }\end{array}$ \\
\cline { 2 - 6 } & Topic of interest & $\begin{array}{l}\text { Doesn't change, but } \\
\text { it's altered/modified } \\
\text { during a session }\end{array}$ & Create & $\begin{array}{l}\text { Product development } \\
\text { as a whole (project } \\
\text { assignment) }\end{array}$ & Project leader \\
\hline
\end{tabular}

\begin{tabular}{|c|c|c|c|c|c|}
\hline$P$ & Object & Frequency & Source & Allocation & Criteria \\
\hline \multirow[t]{4}{*}{2} & Truck & $\begin{array}{l}\text { Almost every session, } \\
\text { depends on the topic } \\
\text { at hand }\end{array}$ & $\begin{array}{l}\text { Create } \\
\text { Import }\end{array}$ & Project team & Time / costs \\
\hline & Avatar & $\begin{array}{l}\text { Almost never, to make } \\
\text { sure you get familiar } \\
\text { with the personas }\end{array}$ & Built-in & - & $\begin{array}{l}\text { Should be able to } \\
\text { recognise them }\end{array}$ \\
\hline & World & $\begin{array}{l}\text { Depends on the topic, } \\
\text { every now and then }\end{array}$ & $\begin{array}{l}\text { Create } \\
\text { Built-in }\end{array}$ & Project team & Time / costs \\
\hline & Topic of interest & Each session & Create & Project team & $\begin{array}{l}\text { Should be interactive } \\
\rightarrow \text { Time / costs }\end{array}$ \\
\hline
\end{tabular}

\begin{tabular}{|c|c|c|c|c|c|}
\hline$P$ & Object & Frequency & Source & Allocation & Criteria \\
\hline \multirow[t]{3}{*}{3} & Truck & $\begin{array}{l}\text { Depends on the } \\
\text { project phase (level of } \\
\text { detail needed) and } \\
\text { purpose }\end{array}$ & Import & Vehicle definition & $\begin{array}{l}\text { Required level of } \\
\text { detail }\end{array}$ \\
\hline & Avatar & $\begin{array}{l}\text { Only once, then re-use } \\
\text { the personas for a } \\
\text { long period of time }\end{array}$ & Built-in & Vehicle definition & \\
\hline & World & $\begin{array}{l}\text { Make a fixed set of } \\
\text { traffic scenarios, such } \\
\text { as winter/summer, } \\
\text { test tracks, known } \\
\text { routes }\end{array}$ & $\begin{array}{l}\text { Import } \\
\text { Built-in }\end{array}$ & Vehicle definition & $\begin{array}{l}\text { Required level of } \\
\text { detail, and required } \\
\text { scenarios }\end{array}$ \\
\hline
\end{tabular}




\section{Tool selection (continued)}

\begin{tabular}{|l|l|l|l|l|l|}
\hline $\mathrm{P}$ & Object & Frequency & Source & Allocation & Criteria \\
\hline 4 & Truck & $\begin{array}{l}\text { Once for every cabin } \\
\text { generation. We would } \\
\text { need several trucks to } \\
\text { represent different } \\
\text { cabin types }\end{array}$ & Import & Cabin /chassis group & $\begin{array}{l}\text { Detailed shapes and } \\
\text { styling, should be } \\
\text { recognisable as our } \\
\text { trucks }\end{array}$ \\
\cline { 2 - 6 } & Avatar & $\begin{array}{l}\text { Once, three variations } \\
\text { (p5/p50/p95), and } \\
\text { maybe two sub types }\end{array}$ & $\begin{array}{l}\text { Need to be able to } \\
\text { edit/modify existing } \\
\text { avatar models }\end{array}$ & $\begin{array}{l}\text { Vehicle definition or } \\
\text { external partner }\end{array}$ & $\begin{array}{l}\text { Realistic movement } \\
\text { envelope, simple } \\
\text { controls, natural } \\
\text { movements, } \\
\text { composite } \\
\text { movements }\end{array}$ \\
\cline { 2 - 6 } & World & $\begin{array}{l}\text { Create one basic } \\
\text { world, we'll add } \\
\text { specific situations } \\
\text { when needed }\end{array}$ & Create & $\begin{array}{l}\text { Vehicle definition or } \\
\text { external partner }\end{array}$ & $\begin{array}{l}\text { Interaction with } \\
\text { traffic, context should } \\
\text { be interactive where } \\
\text { needed }\end{array}$ \\
\cline { 2 - 6 } & Topic of interest & $\begin{array}{l}\text { Topics are added each } \\
\text { session, every topic is } \\
\text { saved in a library }\end{array}$ & Create & $\begin{array}{l}\text { Import } \\
\text { Cabin department } \\
\text { whenever possible, } \\
\text { otherwise vehicle } \\
\text { definition or external } \\
\text { partner }\end{array}$ & $\begin{array}{l}\text { Interaction with } \\
\text { context when needed } \\
\text { for the topic of } \\
\text { interest }\end{array}$ \\
\hline
\end{tabular}

\begin{tabular}{|l|l|l|l|l|l|}
\hline & Object & Frequency & Source & Allocation & Criteria \\
\hline 5 & Truck & $\begin{array}{l}\text { An extensive set } \\
\text { should be created } \\
\text { once, updated when } \\
\text { we have new trucks }\end{array}$ & Import & $\begin{array}{l}\text { 3D modelling, possibly } \\
\text { an external partner } \\
\text { for the first set. After } \\
\text { that it should be } \\
\text { modified through } \\
\text { internal tool }\end{array}$ & $\begin{array}{l}\text { Visual quality, level of } \\
\text { realism }\end{array}$ \\
\hline Avatar & $\begin{array}{l}\text { Only once, because } \\
\text { personas need to } \\
\text { become familiar } \\
\text { throughout the } \\
\text { company }\end{array}$ & Built-in & $\begin{array}{l}\text { Marketing defines } \\
\text { core target group, } \\
\text { modelling in } \\
\text { collaboration with } \\
\text { vehicle definition }\end{array}$ & $\begin{array}{l}\text { We should be able to } \\
\text { distinguish them from } \\
\text { eachother } \\
\text { They should be } \\
\text { interactive }\end{array}$ \\
\cline { 2 - 6 } & Once & Built-in & $\begin{array}{l}\text { Maybe an existing } \\
\text { environment from a } \\
\text { game or simulator }\end{array}$ & $\begin{array}{l}\text { Should provide } \\
\text { sufficient context, } \\
\text { visual quality is } \\
\text { important, not } \\
\text { functional so } \\
\text { shouldn't take too } \\
\text { much effort/time }\end{array}$ \\
\hline
\end{tabular}




\section{B.3 Cross-company evaluation 2}

In the second cross-company evaluation the Virtual Persona application, originally developed for company $\mathrm{B}$, was demonstrated to and evaluated by companies $\mathrm{A}$ and $\mathrm{C}$. After presenting and experiencing the application prototypes, the participants filled out the evaluation forms included in appendix B.3.1. The results of this evaluation are included in appendix B.3.2.

- All forms and answers have been translated from Dutch.

\section{B.3.1 CCE2 forms}

REPAR-B // Cross-Company Evaluation II

Company / companies:

Group members:

This form contains questions that help you 'rranslate' the Virtual Personas application into

The form consists of three parts:

Part $1 / /$ Using the Virtual Environment
Part $2 / /$ The Virtual Environment itself
Part $3 / /$ Creating the Virtual Environment
PART 1 // USING THE VIRTUAL ENVIRONMENT

Imagine you created the virtual environment according to your requirements, how woul you use it in practice, and how would it help you? These questions are about actually
using the virtual environment in a user study. What would be the main purpose of this using the virtual environment in a
study, who would be involved, etc.?

\begin{tabular}{|l|l}
1.1 & Our test subjects/participants would be (multiple answers allowed)
\end{tabular}

End-users

End-user representatives

Decision makers

Us (designers, engineers)

Other departments within the company

Something else:

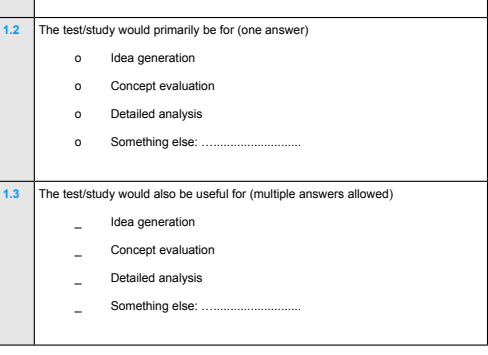




\section{CCE2 forms (continued)}

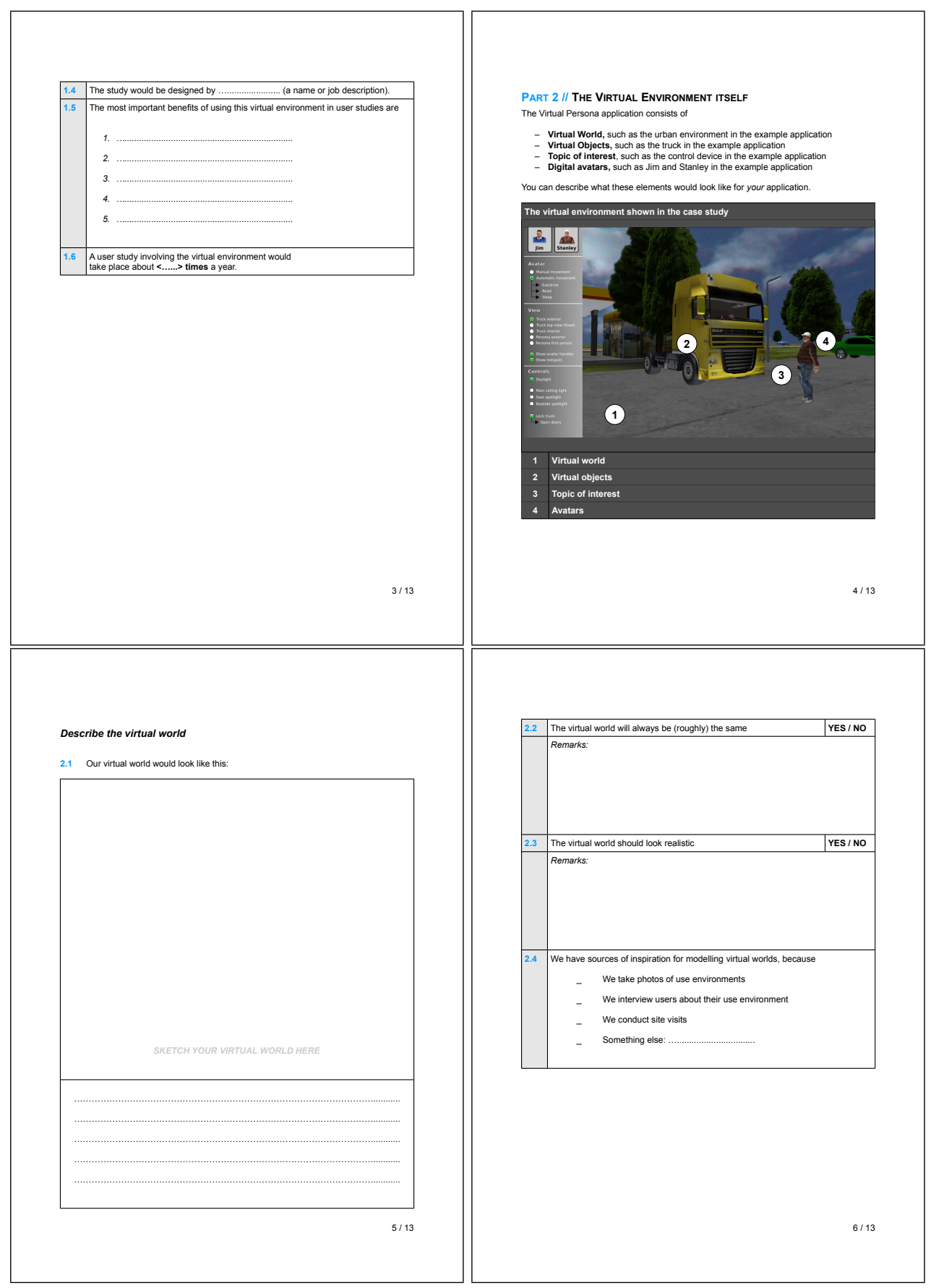




\section{CCE2 forms (continued)}

Describe virtual objects

2.5 What virtual objects can be found in your virtual world (in order of priority)?

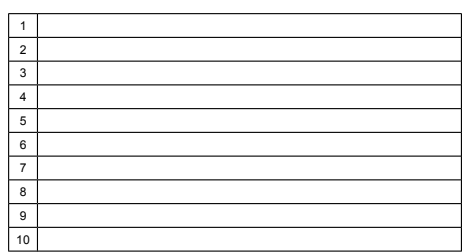

\begin{tabular}{|l|l}
\hline 2.6 & Virtual objects should look realistic \\
\hline & Renets
\end{tabular}

YES / NO

\begin{tabular}{l|l|}
\hline 2.6 & Virtual ob \\
\hline & Remarks. \\
\hline &
\end{tabular}

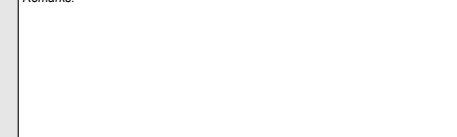

Describe the avatars

Describe the desired virtual avatars that represent your personas

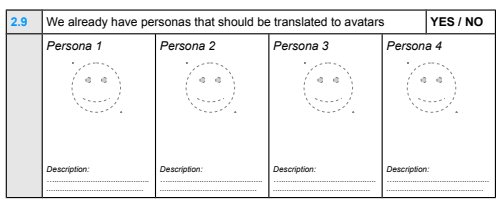

\begin{tabular}{|l|l|l|}
\hline 2.10 & We should be able to quickly change avatars, because we use a lot & YES / NO \\
\hline
\end{tabular} Remarks:

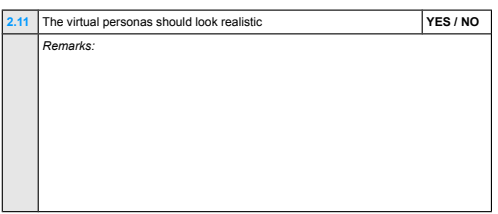

Describe the topic(s) of interest

2.7 Which topic(s) of interest could be found in your virtual world?
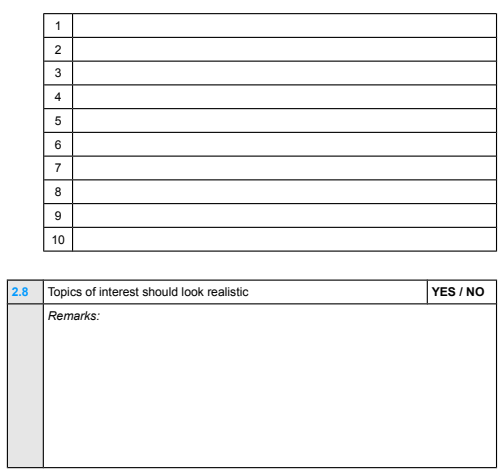

Compare your virtual world to the one presented in the case study

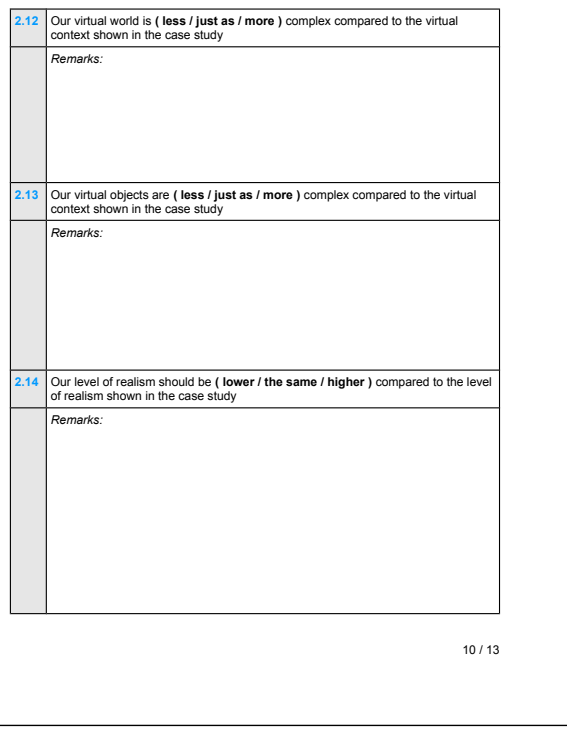




\section{CCE2 forms (continued)}

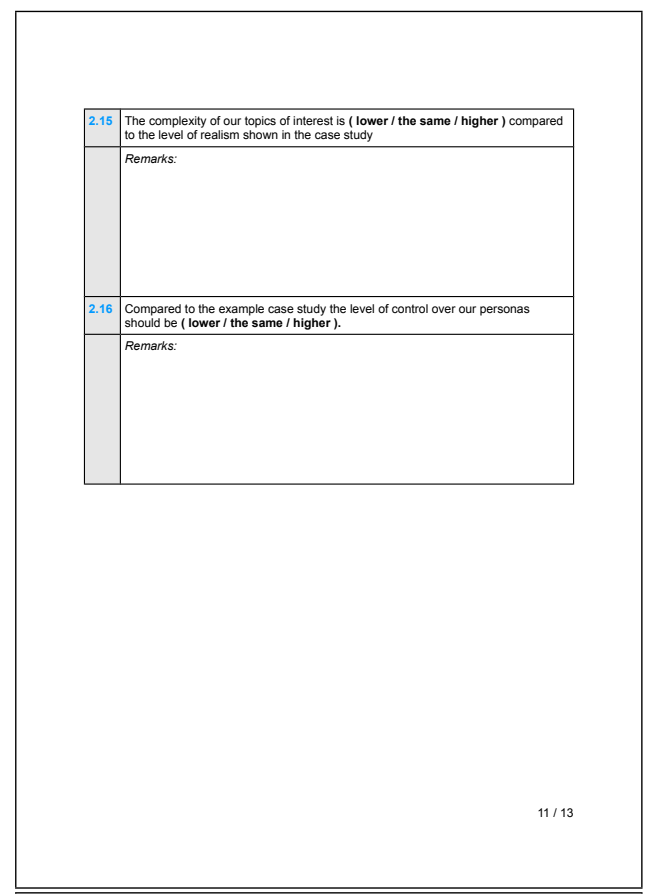

PART 3 // CREating the Virtual EnVironment

This part of the form investigates the realisation of the desired application; what kind of

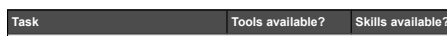

\begin{tabular}{|l|}
\hline 3.1 VIRTUAL WORLD \\
\hline Creating the virtual world \\
\hline
\end{tabular}

Yesino

\begin{tabular}{|c|c|c|}
\hline Creating the virtual world & $\begin{array}{r}\text { YES / NO } \\
\text { Tool: }\end{array}$ & YES / NO \\
\hline
\end{tabular}

\begin{tabular}{|l|l|l|}
\hline $\begin{array}{l}\text { - If tools are not available, would } \\
\text { the tools shown in the case } \\
\text { studd be suitable for you? }\end{array}$ & \\
\hline$\cdot \begin{array}{l}\text { If skills are not avalable, how } \\
\text { would you solve this? }\end{array}$ & \\
\hline 3.2 & VIRTUELE OBJECTEN \\
\hline C
\end{tabular}

3.2 VIRTUELE OBJECTEN

\begin{tabular}{|c|c|c|}
\hline Creating virtual objects & YES / NO & YES/NO \\
\hline $\begin{array}{l}\text { If tools are not available, would } \\
\text { the tools shown in the case } \\
\text { study be suitable for you? }\end{array}$ & & \\
\hline $\begin{array}{l}\text { If skills are not available, how } \\
\text { would you solve this? }\end{array}$ & & \\
\hline \multicolumn{3}{|l|}{$\begin{array}{ll}3.3 & \text { PRODUCTCONCEPTEN }\end{array}$} \\
\hline Creating topics of interest & 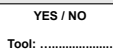 & 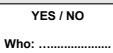 \\
\hline $\begin{array}{l}\text { If tools are not available, would } \\
\text { the tools shown in the case } \\
\text { study be suitable for you? }\end{array}$ & & \\
\hline $\begin{array}{l}\text { If skills are not available, how } \\
\text { would you solve this? }\end{array}$ & & \\
\hline $3.4 \quad$ AVATARS & & \\
\hline
\end{tabular}

\begin{tabular}{|l|c|c|}
\hline Creating virtual avatars & $\begin{array}{c}\text { YES / NO } \\
\text { Tool: ................... }\end{array}$ & $\begin{array}{c}\text { YES / NO } \\
\text { Who: ................... }\end{array}$ \\
\hline $\begin{array}{l}\text {. If tools are not available, would } \\
\text { the tools shown in the case } \\
\text { study be suitable for you? }\end{array}$ & \\
\hline $\begin{array}{l}\text {. If skills are not available, how } \\
\text { would you solve this? }\end{array}$ & \\
\hline
\end{tabular}




\section{B.3.2 CCE2 results}

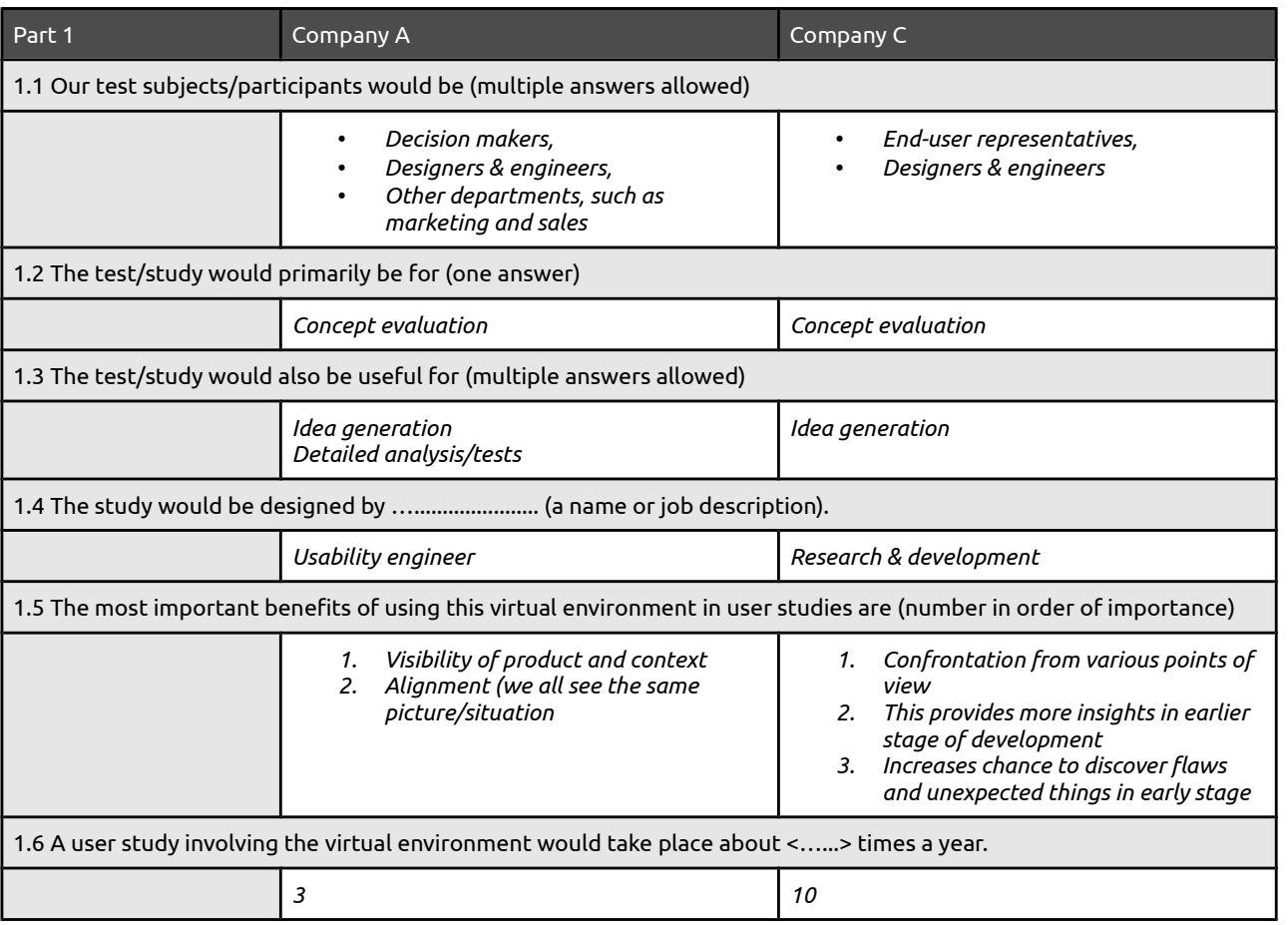




\section{CCE2 results (continued)}

\begin{tabular}{|c|c|c|}
\hline Part 2 & Company A & Company C \\
\hline \multicolumn{3}{|c|}{ 2.1 The virtual world would consist of... } \\
\hline & $\begin{array}{l}\text { Print/repro environment with personas, } \\
\text { includes a workstation, printers, cantine, } \\
\text { coffee corner }\end{array}$ & $\begin{array}{l}\text { An average food processing plant, in which our } \\
\text { (and other) machines are positioned. Personas } \\
\text { walk around the environment. }\end{array}$ \\
\hline \multicolumn{3}{|c|}{ 2.2 This virtual world is roughly the same for each test/study we do } \\
\hline & No & Yes, just about \\
\hline \multicolumn{3}{|c|}{ 2.3 This virtual world should look realistic } \\
\hline & $\begin{array}{l}\text { Yes; convincing and recognisable for designers, } \\
\text { not too many details }\end{array}$ & $\begin{array}{l}\text { Yes/no, it's not about textures (details) but } \\
\text { more about physical properties/characteristics }\end{array}$ \\
\hline \multicolumn{3}{|c|}{ 2.4 We have sources of inspiration for modelling virtual worlds, because } \\
\hline & $\begin{array}{ll}\text { - } & \text { We take photos of use environments } \\
\text { - } & \text { We interview users } \\
\text { - } & \text { We have personas }\end{array}$ & $\begin{array}{ll}\text { - } & \text { We take photos of use environments } \\
\text { - } & \text { We interview users } \\
\text { - } & \text { We have CAD models }\end{array}$ \\
\hline \multicolumn{3}{|c|}{ 2.5 List of virtual objects } \\
\hline & $\begin{array}{l}\text { Copier / printer, stacks of paper, building, PC, } \\
\text { desk, coffee corner }\end{array}$ & $\begin{array}{l}\text { Basic equipment, tool sets, secondary } \\
\text { equipment, replacement tools, other (not our) } \\
\text { equipment }\end{array}$ \\
\hline \multicolumn{3}{|c|}{ 2.6 These objects should closely resemble the look and feel of real-life objects } \\
\hline & Yes; Recognisable & Yes; More realistic than the environment \\
\hline \multicolumn{3}{|c|}{ 2.7 Which virtual topics of interest should be available? } \\
\hline & Smartphone, printer, paper trolley & $\begin{array}{l}\text { Smartphone or tablet, tool sets, replacement } \\
\text { tools, touch panel }\end{array}$ \\
\hline \multicolumn{3}{|c|}{ 2.8 Our virtual topics of interest should look realistic } \\
\hline & Yes/no; Recognisable & Yes \\
\hline \multicolumn{3}{|c|}{ 2.9 We already have personas that we can use in this application } \\
\hline & Yes, we have personas for each project & $\begin{array}{l}\text { No, we'll define personas based on functions } \\
\text { related to the machines (e.g. cleaner, operator, } \\
\text { supervisor, engineers) }\end{array}$ \\
\hline \multicolumn{3}{|c|}{ 2.10 Virtal personas need to be adaptable } \\
\hline & Yes & No \\
\hline \multicolumn{3}{|c|}{ 2.11 Virtal personas should look realistic } \\
\hline & $\begin{array}{l}\text { Recognisable identity, e.g. age should be easy } \\
\text { to see }\end{array}$ & No, just recognisable (their function/job) \\
\hline \multicolumn{3}{|c|}{2.12 Our virtual world is ( less / just as / more ) complex compared to the virtual context shown in the case study } \\
\hline & - & More \\
\hline
\end{tabular}




\section{CCE2 results (continued)}

2.13 Our virtual objects are ( less / just as / more ) complex compared to the virtual context shown in the case study \begin{tabular}{l|l} 
More; we need to include machines from & More; we want to include accessibility of
\end{tabular} competitors $\quad$ machine components, tools, etc.

2.14 Our level of realism should be (lower / the same / higher ) compared to the level of realism shown in the case study

\begin{tabular}{|l|l} 
The same & $\begin{array}{l}\text { The same; personas may be less complex or } \\
\text { detailed }\end{array}$
\end{tabular}

2.15 The complexity of our topics of interest is ( lower / the same / higher ) compared to the level of realism shown in the case study

\begin{tabular}{|l|l|l|}
\hline & Higher & - \\
\hline 2.16 Compared to the example case study the level of control over our personas should be (lower / the same / higher ). \\
\hline & The same; this depends on the environment & - \\
\hline
\end{tabular}

\begin{tabular}{|c|c|c|}
\hline Part 3 & Company A & Company C \\
\hline \multicolumn{3}{|l|}{ 3.1 Virtual world } \\
\hline $\begin{array}{l}\text { Are Virtual world } \\
\text { modelling tools and } \\
\text { skills available? If not, } \\
\text { how would you solve } \\
\text { this? }\end{array}$ & Yes & - \\
\hline \multicolumn{3}{|l|}{3.2 Virtual objects } \\
\hline $\begin{array}{l}\text { Are Virtual Object } \\
\text { modelling tools and } \\
\text { skills available? If not, } \\
\text { how would you solve } \\
\text { this? }\end{array}$ & Yes, Product designers & - \\
\hline \multicolumn{3}{|l|}{ 3.3 Topics of interest } \\
\hline $\begin{array}{l}\text { Are Topics of interest } \\
\text { modelling tools and } \\
\text { skills available? If not, } \\
\text { how would you solve } \\
\text { this? }\end{array}$ & $\begin{array}{l}\text { Yes, game engines can be used by our digital } \\
\text { prototypers }\end{array}$ & - \\
\hline \multicolumn{3}{|l|}{ 3.4 Avatars } \\
\hline $\begin{array}{l}\text { Are Avatar modelling } \\
\text { tools and skills } \\
\text { available? If not, how } \\
\text { would you solve this? }\end{array}$ & $\begin{array}{l}\text { Don't know, but digital prototypers should be } \\
\text { able to do this }\end{array}$ & - \\
\hline
\end{tabular}



C Case Study 3 Data 


\section{C.1 Application validation}

The application validation session of case study 3 involved two groups of five participants (see table C.1) who were asked to use the Virtual Annotation application in a design review session. After the session participants filled out the evaluation form included in appendix C.1.1. The results of this evaluation are included in appendix C.1.2.

- All forms and answers have been translated from Dutch.

\begin{tabular}{lll}
\hline Group & Participant & Function \\
\hline G1 & P1 & R\&D \\
& P2 & Technologist \\
& P3 & Assembly engineer \\
& P4 & Technologist \\
& P5 & R\&D \\
\hline G2 & P1 & R\&D \\
& P2 & Assembly engineer \\
& P3 & TSM \\
& P4 & Engineering \\
& P5 & RTC \\
\hline
\end{tabular}

Table C.1 List of participants and their labels. 
C.1.1 Application validation form

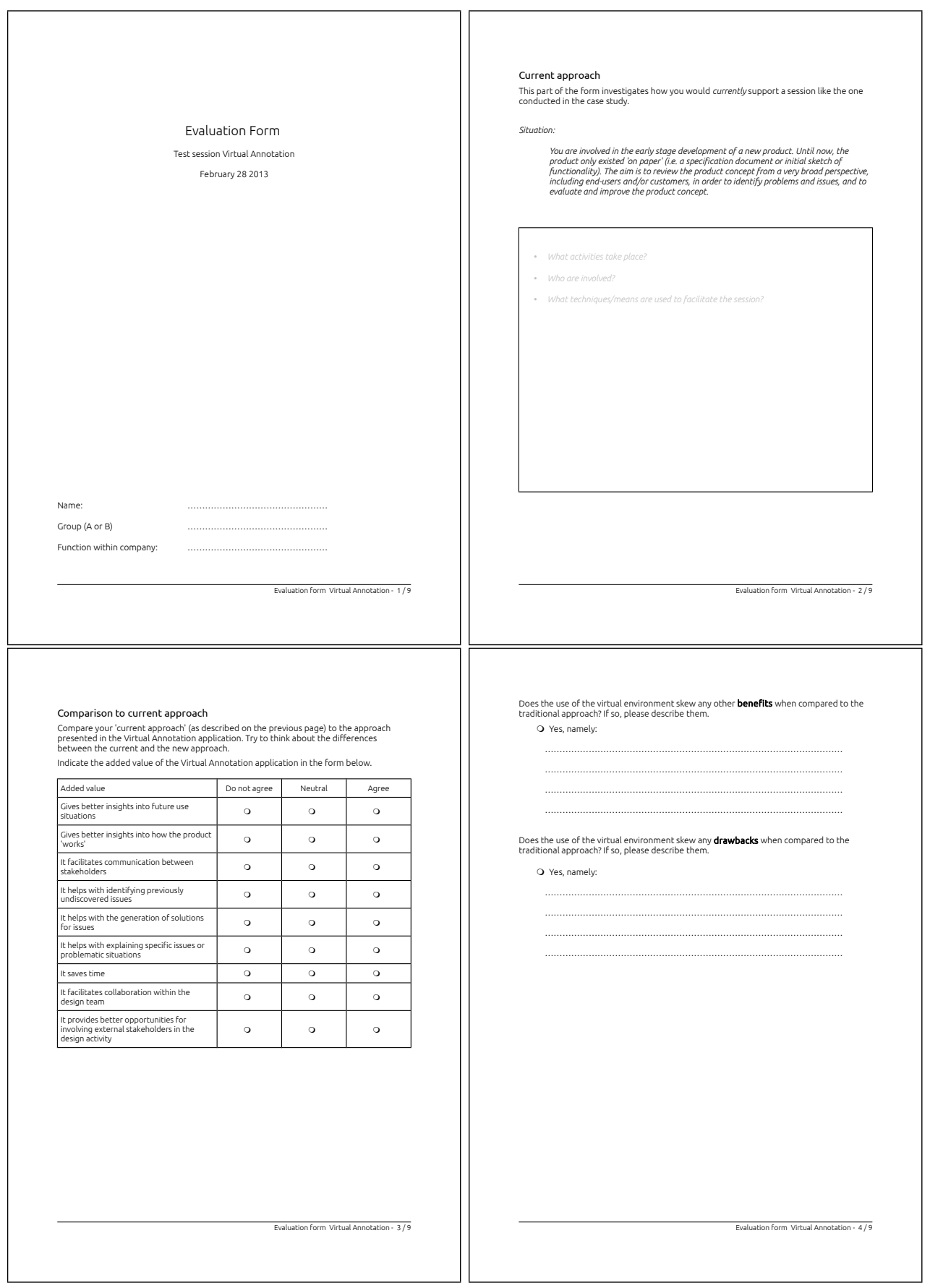




\section{Application validation form (continued)}

The Virtual Environment The following questions are about the virtual environment as presented in the test
session. 1 is is no longer about a comparison between the traditional approach and the new

Functionality

The test/study would primarily be for (one answer)

O Generating new solutions

O Visualisation and presentation of solutions and problem situations

O Evaluation of new solutions or product concepts

o something else: ..........

The following table lists the core functions of the application. Please indicate whether you

\begin{tabular}{|c|c|c|}
\hline Functionality & \begin{tabular}{|l|} 
Prionity \\
\end{tabular} & $\begin{array}{l}\text { Implementation OK? If not, please suggest } \\
\text { improvements }\end{array}$ \\
\hline 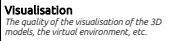 & $1 / 2 / 3 / 4 / 4 / 5$ & \\
\hline 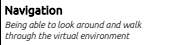 & $1 / 2 / 3 / 4 / 5$ & \\
\hline $\begin{array}{l}\text { Notes } \\
\text { Being able to oddd notes, including } \\
\text { prionity, cotegong, etc }\end{array}$ & $1 / 2 / 3 / 4 / 45$ & \\
\hline
\end{tabular}

Evaluation form Virtual Annotation - $5 / 9$

Interaction with the virtual environment

You experienced 2 forms of interaction with the virtual environment:

1. via a moderator

2. via individual input devices

What are the most relevant benefits and drawbacks of each of these forms of interaction?

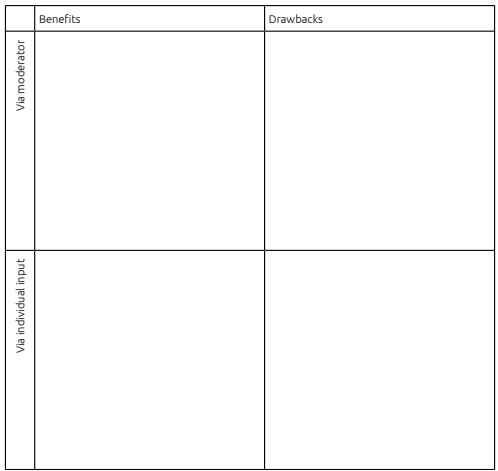

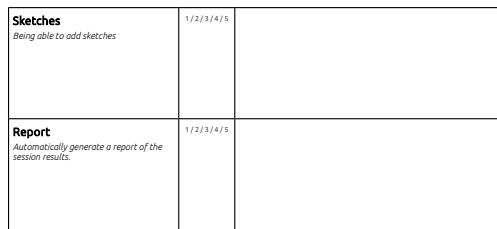

Any other suggestions that would improve the presented application?

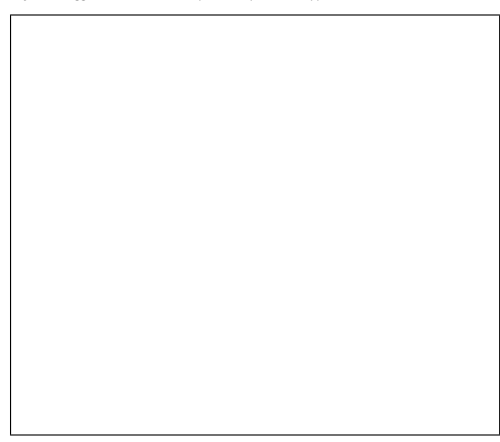

Evaluation form Virtual Annotation - 6/9

Did the form of interaction stimulate participation in the session?

\begin{tabular}{|l|c|c|}
\hline & Via moderator & Via individual input \\
\hline Notat all & 0 & 0 \\
\hline Only in the beginning & 0 & 0 \\
\hline Sometimes & 0 & 0 \\
\hline Yes & 0 & 0 \\
\hline
\end{tabular}

Did the form of interaction stimulate group collaboration?

\begin{tabular}{|l|c|c|}
\hline & Via moderator & Via individual input \\
\hline Not at all & $\circ$ & $\circ$ \\
\hline Only in the beginning & 0 & $\circ$ \\
\hline Sometimes & $\circ$ & 0 \\
\hline Yes & 0 & 0 \\
\hline
\end{tabular}

Which form of input do you prefer, and why?

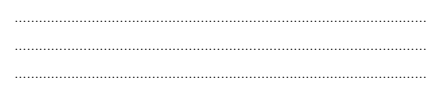

What influence does the form of input have on the session? 
Application validation form (continued)

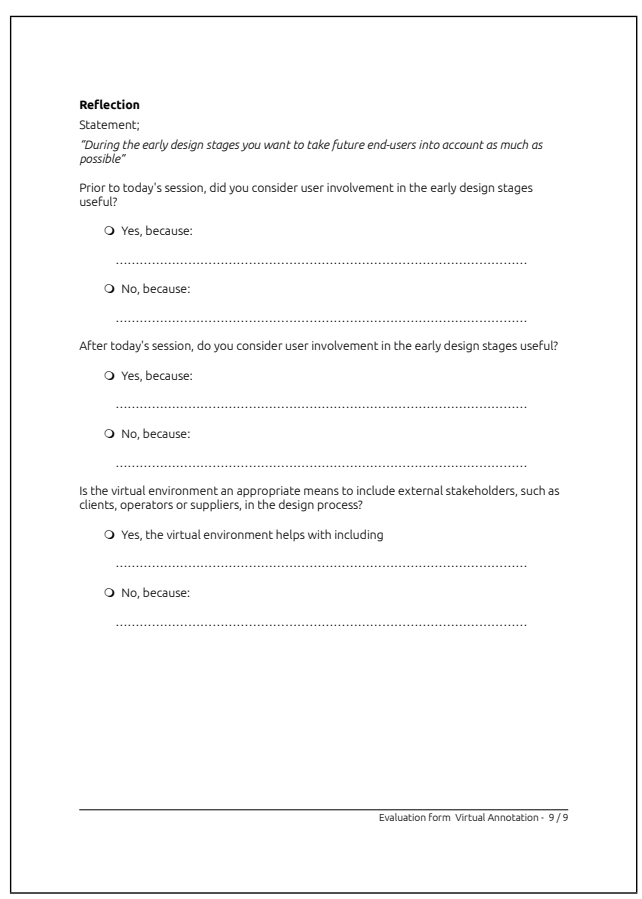




\section{C.1.2 Application validation results}

\begin{tabular}{|c|c|c|c|c|c|}
\hline Part 1 & G1.P1 & G1.P2 & G1.P3 & G1.P4 & G1.P5 \\
\hline \multicolumn{6}{|l|}{ Current Approach } \\
\hline $\begin{array}{l}1.1 \text { How would you } \\
\text { currently support a } \\
\text { session like the one } \\
\text { conducted in the case } \\
\text { study. }\end{array}$ & $\begin{array}{l}\text { Market research, concept } \\
\text { definition, establish } \\
\text { requirements. Define } \\
\text { modularity, design each module. } \\
\text { Then the first detailing phase. } \\
\text { Involves salese, project } \\
\text { management, engineering, } \\
\text { montage, work preparation } \\
\text { crew and suppliers }\end{array}$ & $\begin{array}{l}\text { Gathering insights from end- } \\
\text { users as well as internal sources } \\
\text { and competitors. Make a list of } \\
\text { design criteria for new product. } \\
\text { Make first sketches, and a } \\
\text { prototype. Test and adapt } \\
\text { where needed, then do a } \\
\text { production test. Hand over after } \\
\text { succesful test. Hist. } \\
\text { This involves R\&D, sales, } \\
\text { technologists and montage. }\end{array}$ & $\begin{array}{l}\text { Collect as many drawings as } \\
\text { possible to get info about the } \\
\text { new product. Then we make } \\
\text { new technical drawings and } \\
\text { prototypes. This involves R\&D } \\
\text { and product/process engineers }\end{array}$ & $\begin{array}{l}\text { Determine unit functionality, } \\
\text { see what we can do now } \\
\text { already. Find out how } \\
\text { competitors work, do research } \\
\text { on how similar problems are } \\
\text { solved. Set up a multi- } \\
\text { disciplinary team, and } \\
\text { determine market needs. Try to } \\
\text { save costs by combining } \\
\text { multiple functions in one unit. } \\
\text { Results in better ROI for client. }\end{array}$ & $\begin{array}{l}\text { We interview users/clients, and } \\
\text { split up the unit in functions. } \\
\text { Define scenarios of use, and } \\
\text { determine specifications based } \\
\text { on existing knowledge. We test } \\
\text { boundary conditions, and } \\
\text { identify additional } \\
\text { requirements. Then we use } \\
\text { simple sketches to trigger } \\
\text { response from clients about the } \\
\text { new design. This response is } \\
\text { (somehow) documented. }\end{array}$ \\
\hline \multicolumn{6}{|c|}{ 1.2 Indicate the added value of the Virtual Annotation application in the form below. } \\
\hline $\begin{array}{l}\text { Gives better insights into } \\
\text { future use situations }\end{array}$ & Yes & Yes & Yes & Yes & Yes \\
\hline $\begin{array}{l}\text { Gives better insights into how } \\
\text { the product' works' }\end{array}$ & Neutral & Neutral & Neutral & Neutral & Yes \\
\hline $\begin{array}{l}\text { It facilitates communication } \\
\text { between stakeholders }\end{array}$ & Yes & Yes & Yes & Yes & Neutral \\
\hline $\begin{array}{l}\text { It helps with identifying } \\
\text { previously undiscovered } \\
\text { issues }\end{array}$ & Yes & Neutral & Neutral & Yes & Yes \\
\hline $\begin{array}{l}\text { It thelps with the generation } \\
\text { of solutions for issues }\end{array}$ & Neutral & Yes & Yes & Yes & Neutral \\
\hline $\begin{array}{l}\text { It helps with explaining } \\
\text { specific issues or problematic } \\
\text { situations }\end{array}$ & Yes & Yes & Yes & Yes & Yes \\
\hline It saves time & Neutral & Neutral & Neutral & Yes & No \\
\hline $\begin{array}{l}\text { It facilitates collaboration } \\
\text { within the design team }\end{array}$ & Yes & Yes & Yes & Yes & Neutral \\
\hline $\begin{array}{l}\text { Itprovides better } \\
\text { opportunnities for rinvolving } \\
\text { external stakeholders in the } \\
\text { design activity }\end{array}$ & Yes & Yes & Yes & Yes & Yes \\
\hline
\end{tabular}

\begin{tabular}{|l|l|l|l|l|l|}
\hline 1.3 Does the use of the virtual environment skew any other benefits when compared to the traditional approach? \\
\hline & $\begin{array}{l}\text { Yes, it allows to identify } \\
\text { problems in an early design } \\
\text { stage. It also acts as a } \\
\text { structured collector of ideas. }\end{array}$ & $\begin{array}{l}\text { Yes, as a sales tool for layout } \\
\text { and placement in a building }\end{array}$ & $\begin{array}{l}\text { Yes, better insight/visualisation } \\
\text { of the unit and its functions } \\
\text { Problems and notes are directly } \\
\text { shown to all users in the group }\end{array}$ & $\begin{array}{l}\text { Yes, it allows to quickly give a } \\
\text { client a visualisation of our } \\
\text { machines. Could be connected } \\
\text { to p prices, allowing for making } \\
\text { new offers quickly }\end{array}$ & $\begin{array}{l}\text { Yes, identifies technical } \\
\text { problems with installation on } \\
\text { site in the early design stages }\end{array}$ \\
\hline 1.4 Does the use of the virtual environment skew any drawbacks when compared to the traditional approach? & $\begin{array}{l}\text { Yes, You'll need good hardware } \\
\text { and reliable software }\end{array}$ & $\begin{array}{l}\text { Yes, when the tools do not work } \\
\text { properly or are not user friendly, } \\
\text { it may block the discussions. } \\
\text { This is a temporary problem. }\end{array}$ \\
\hline & $\begin{array}{l}\text { Not really, can only see } \\
\text { advantages right now }\end{array}$ & $\begin{array}{l}\text { It's possible that customers are } \\
\text { involved too early, when we } \\
\text { don't want it yet. For instance in } \\
\text { prototyping stages. }\end{array}$ & - &
\end{tabular}


Application validation results (continued)

\begin{tabular}{|c|c|c|c|c|c|}
\hline Part 1 & G2.P1 & G2.P2 & G2.P3 & G2.P4 & G.P5 \\
\hline \multicolumn{6}{|l|}{ Current Approach } \\
\hline $\begin{array}{l}1.1 \text { How would you } \\
\text { currently support a } \\
\text { session like the one } \\
\text { conducted in the case } \\
\text { study. }\end{array}$ & $\begin{array}{l}\text { Activities: setting/configuring } \\
\text { the machine, change tool parts, } \\
\text { cleaning, maintanance. } \\
\text { Involves operators, service } \\
\text { engineers and cleaners. } \\
\text { Means: talk to internal client } \\
\text { representative about a } \\
\text { particular machine. Watch users } \\
\text { work. }\end{array}$ & $\begin{array}{l}\text { I would invite the user(s) to } \\
\text { come and see the machine. We } \\
\text { often see that every customer } \\
\text { has his/her opinion, we need to } \\
\text { find a middle way. Need to think } \\
\text { about how to (wet) clean the } \\
\text { machine, or design it in such a } \\
\text { way that this is possible. }\end{array}$ & $\begin{array}{l}\text { Involve users in the design } \\
\text { process; periodic interviews, } \\
\text { using sketches or visuals to } \\
\text { show units to the user, } \\
\text { identifying unique selling points } \\
\text { of the new machine wrt the } \\
\text { older machine, and identify cost } \\
\text { aspects. }\end{array}$ & $\begin{array}{l}\text { Interview future users, make a } \\
\text { coarse design to identify } \\
\text { discussion points. Use own } \\
\text { experience to create a first (3d) } \\
\text { model. Capture responses and } \\
\text { remarks in a report, make } \\
\text { inventory of the issues that } \\
\text { affect (future) practical use of } \\
\text { the machine. }\end{array}$ & $\begin{array}{l}\text { Make an inventory of end-user } \\
\text { requirements regarding the } \\
\text { machine. Interview session to } \\
\text { discuss and compare to old } \\
\text { machine, identify existing } \\
\text { problems. Example; use VR to } \\
\text { discuss current machines. Visit } \\
\text { companies of the end-user to } \\
\text { discuss techniques, prices, etc. }\end{array}$ \\
\hline $\begin{array}{l}\text { Gives better insights into } \\
\text { future use situations }\end{array}$ & Yes & Yes & Yes & Neutral & Yes \\
\hline $\begin{array}{l}\text { Gives better insights into how } \\
\text { the product 'works' }\end{array}$ & $\begin{array}{l}\text { Neutral, depends on the } \\
\text { complexity }\end{array}$ & Neutral & Yes & Neutral & Yes \\
\hline $\begin{array}{l}\text { It facilitates communication } \\
\text { between stakeholders }\end{array}$ & Yes & Yes & Neutral & Yes & Yes \\
\hline $\begin{array}{l}\text { It helps with explaining } \\
\text { specific issues or problematic } \\
\text { situations }\end{array}$ & Yes & Yes & Yes & Yes & Yes \\
\hline It saves time & $\begin{array}{l}\text { Yes, it triggers more discussion, } \\
\text { so takes time, but information is } \\
\text { valuable }\end{array}$ & Yes & Neutral & Yes & Neutral \\
\hline $\begin{array}{l}\text { It facilitates collaboration } \\
\text { within the design team }\end{array}$ & Yes & Yes & Yes & Yes & Yes \\
\hline $\begin{array}{l}\text { It provides better } \\
\text { opportunities for involving } \\
\text { external stakeholders in the } \\
\text { design activity }\end{array}$ & Yes & Yes & Yes & Yes & Yes \\
\hline
\end{tabular}

\begin{tabular}{|c|c|c|c|c|c|}
\hline & $\begin{array}{l}\text { Yes, it establishes and maintains } \\
\text { a focus. Participants are } \\
\text { triggered by the constant } \\
\text { representation of the design }\end{array}$ & $\begin{array}{l}\text { Yes, everything is much more } \\
\text { visible, making it easier to } \\
\text { capture remarks. }\end{array}$ & $\begin{array}{l}\text { Yes, spatial view gives better } \\
\text { insight in details }\end{array}$ & $\begin{array}{l}\text { Yes, all notes/responses of users } \\
\text { are captured in a single } \\
\text { document }\end{array}$ & $\begin{array}{l}\text { Yes, as a configuration/design } \\
\text { tool during technical sales } \\
\text { meetings, using standard units } \\
\text { before advanced calculations } \\
\text { start. Visualisation increases the } \\
\text { understanding of the units. }\end{array}$ \\
\hline \multicolumn{6}{|c|}{ 1.4 Does the use of the virtual environment skew any drawbacks when compared to the traditional approach? } \\
\hline & $\begin{array}{l}\text { Yes, the user interface of the } \\
\text { remote devices may keep } \\
\text { participants from participating } \\
\text { in the dicussions (a prototype } \\
\text { issue) }\end{array}$ & $\begin{array}{l}\text { Yes, everything is visualised } \\
\text { 'perfectly', seems that } \\
\text { construction engineers have less } \\
\text { work to do }\end{array}$ & $\begin{array}{l}\text { There may be too many details } \\
\text { that prevent the 'execution' of } \\
\text { the unit }\end{array}$ & $\begin{array}{l}\text { You need to learn how to use } \\
\text { the tool }\end{array}$ & Not at the moment \\
\hline
\end{tabular}




\section{Application validation results (continued)}

\begin{tabular}{|c|c|c|c|c|c|}
\hline Part 2 & G1.P1 & G1.P2 & G1.P3 & G1.P4 & G1.P5 \\
\hline \multicolumn{6}{|l|}{ The Virtual Environment } \\
\hline $\begin{array}{l}2.1 \text { The test/study would } \\
\text { primarily be for (one } \\
\text { answer) }\end{array}$ & $\begin{array}{l}\text { Idea generation, visualisation, } \\
\text { evaluation and allocation of } \\
\text { ideas to people }\end{array}$ & Visualisation & Visualisation & Visualisation & Visualisation \\
\hline \multicolumn{6}{|c|}{ 2.2 Please indicate whether you consider the functionality important } \\
\hline Visualisation & 5 & 5 & 3 & 5 & 3 \\
\hline Navigation & 5 & 4 & $\begin{array}{l}\text { 4; rotating the model shows } \\
\text { more points of interest }\end{array}$ & 5 & 4 \\
\hline Notes & 5 & 4 & $\begin{array}{l}\text { 5; this is very important } \\
\text { prevents repetitive discussions }\end{array}$ & 5 & 5 \\
\hline Sketches & 5 & 4 & 4 & $\begin{array}{l}\text { 3; would work better with a } \\
\text { styles or fixed symbols as in } \\
\text { Word/Excel }\end{array}$ & 4 \\
\hline Report & 5 & 5 & 3 & 5 & 4 \\
\hline \multicolumn{6}{|c|}{ 2.3 Any other suggestions that would improve the presented application? } \\
\hline & - & - & - & $\begin{array}{l}\text { Could be used as a sales tool by } \\
\text { linking the units to prices }\end{array}$ & - \\
\hline \multicolumn{6}{|c|}{ 2.4 What are the most relevant benefits and drawbacks of each of these forms of interaction? } \\
\hline Via moderator & $\begin{array}{l}\text { Advantage: direct contact } \\
\text { Disadvantage: too much } \\
\text { discussion }\end{array}$ & $\begin{array}{l}\text { Advantage: immediately clear in } \\
\text { group sessions }\end{array}$ & $\begin{array}{l}\text { Advantage: group discussion } \\
\text { Disadvantage: takes more time }\end{array}$ & $\begin{array}{l}\text { Advantage: you need a } \\
\text { discussion leader that is able to } \\
\text { save the important data. It } \\
\text { results in a good group } \\
\text { discussion } \\
\text { Disadvantage: takes more time }\end{array}$ & $\begin{array}{l}\text { Advantage: the discussion is } \\
\text { centrallised and controlled; } \\
\text { speeds up the process. } \\
\text { Disadvantage: structure may } \\
\text { reduce creativity }\end{array}$ \\
\hline Via individual input & $\begin{array}{l}\text { Advantage: allows remote } \\
\text { collaboration } \\
\text { Disadvantage: no direct } \\
\text { discussion }\end{array}$ & $\begin{array}{l}\text { Advantage: you can add notes } \\
\text { even after the session, if you } \\
\text { forgot something }\end{array}$ & $\begin{array}{l}\text { Advantage: when you think of } \\
\text { something, just add it } \\
\text { afterwards inatead of } \\
\text { organising a new meeting } \\
\text { Disadvantage: it's more difficult } \\
\text { to indicate you disagree with a } \\
\text { note made by someone else }\end{array}$ & $\begin{array}{l}\text { Advantages; can be done } \\
\text { quickly, and also after the } \\
\text { session; everything is saved. } \\
\text { Could also be used remotely. } \\
\text { Disadvantage: Group doesn't } \\
\text { know what everyone is writing } \\
\text { down. }\end{array}$ & $\begin{array}{l}\text { Advantage: no geographical } \\
\text { constraints } \\
\text { Disadvantage: knowledge from } \\
\text { experts may be blocked when } \\
\text { the user interface doesn't work } \\
\text { as expected }\end{array}$ \\
\hline
\end{tabular}

\begin{tabular}{|c|c|c|c|c|c|}
\hline \multicolumn{6}{|c|}{2.5 Did the form of interaction stimulate participation in the session? } \\
\hline Via moderator & Yes & Yes & Yes & Yes & Yes \\
\hline Via individual input & Sometimes & Sometimes & Sometimes & Yes & Yes \\
\hline Via moderator & Yes & Yes & Yes & Yes & Yes \\
\hline Via individual input & Yes & Sometimes & Only in the beginning & Sometimes & Sometimes \\
\hline \multicolumn{6}{|c|}{2.7 Which form of input do you prefer, and why? } \\
\hline & $\begin{array}{l}\text { Via a moderator, it speeds up } \\
\text { the exchange of opinions }\end{array}$ & $\begin{array}{l}\text { Via moderator, because one } \\
\text { person makes the notes and } \\
\text { sketches, resulting in a clear } \\
\text { report }\end{array}$ & $\begin{array}{l}\text { Via a moderator, this is easier to } \\
\text { exchange ideas and to guide the } \\
\text { discussion. }\end{array}$ & Moderator & $\begin{array}{l}\text { Moderator; should be a } \\
\text { specialist in using the tool, so } \\
\text { participants can focus on the } \\
\text { discussion }\end{array}$ \\
\hline
\end{tabular}


Application validation results (continued)

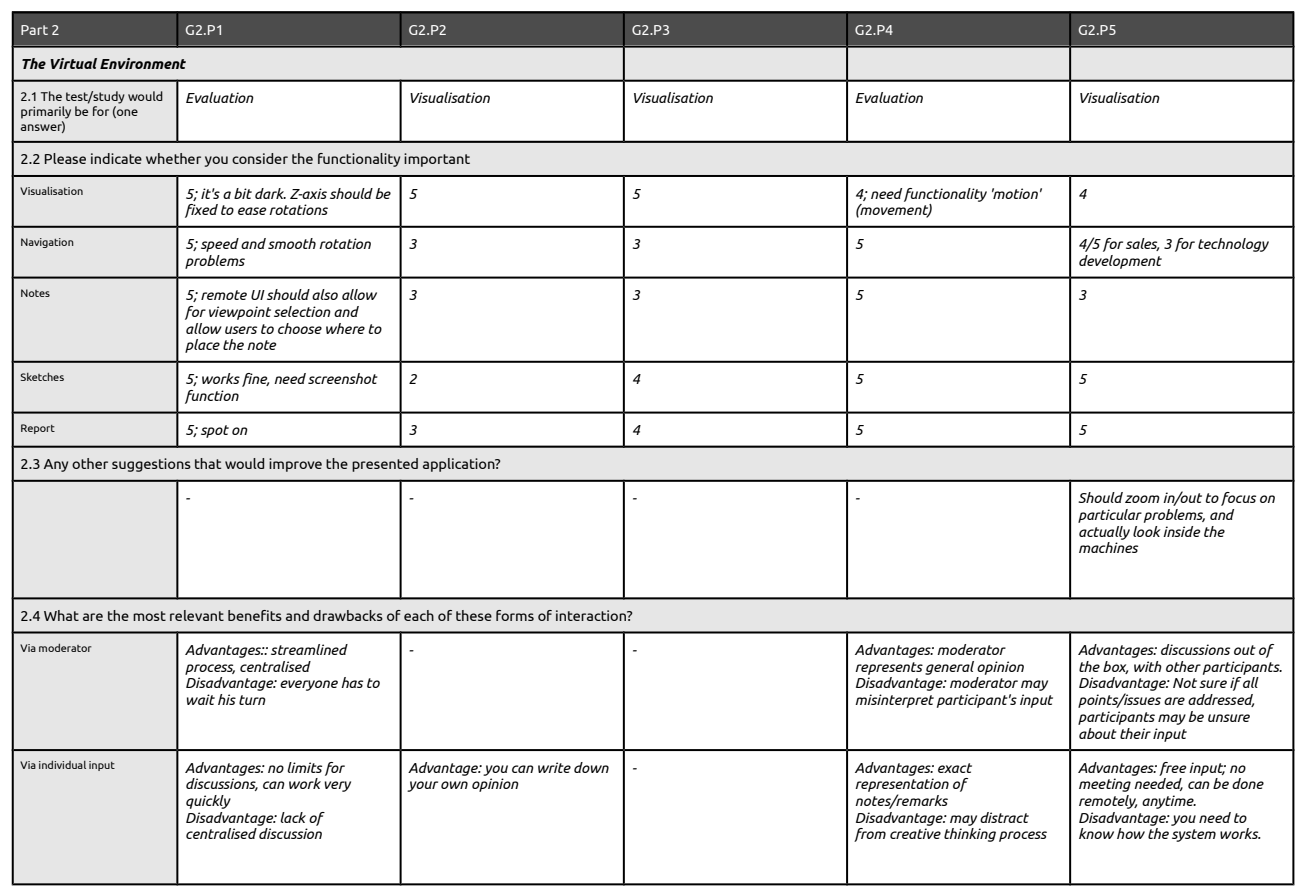

\begin{tabular}{|c|c|c|c|c|c|}
\hline \multicolumn{6}{|c|}{ 2.5 Did the form of interaction stimulate participation in the session? } \\
\hline Via moderator & Yes & Sometimes & Yes & Yes & Yes \\
\hline Via individual input & Yes & Sometimes & - & Yes & Sometimes \\
\hline \multicolumn{6}{|c|}{ 2.6 Did the form of interaction stimulate group collaboration? } \\
\hline Via moderator & Yes & Sometimes & - & Yes & Yes \\
\hline Via individual input & Sometimes & Only in the beginning & - & Sometimes & Sometimes \\
\hline \multicolumn{6}{|c|}{2.7 Which form of input do you prefer, and why? } \\
\hline & $\begin{array}{l}\text { Combination of the two seems } \\
\text { the best option }\end{array}$ & $\begin{array}{l}\text { Moderator, so you can } \\
\text { collaboratively decide on what } \\
\text { to write down }\end{array}$ & - & Moderator & $\begin{array}{l}\text { A moderator allows for } \\
\text { interaction between the } \\
\text { participants, this can boost idea } \\
\text { generation }\end{array}$ \\
\hline
\end{tabular}




\section{Application validation results (continued)}

\begin{tabular}{|c|c|c|c|c|c|}
\hline Part 3 & G1.P1 & G1.P2 & G1.P3 & G1.P4 & G1.P5 \\
\hline \multicolumn{6}{|l|}{ Reflection } \\
\hline $\begin{array}{l}\text { 3.1 Prior to today's } \\
\text { session, did you consider } \\
\text { user involvement in the } \\
\text { early design stages } \\
\text { useful? }\end{array}$ & $\begin{array}{l}\text { Yes, it helps with establishing a } \\
\text { general concensus for the } \\
\text { project direction }\end{array}$ & Yes & Yes & $\begin{array}{l}\text { Yes, things have been } \\
\text { mentioned that were already } \\
\text { decided (technically) but that } \\
\text { may cause problems in practice }\end{array}$ & Yes \\
\hline $\begin{array}{l}\text { 3.2 After today's session, } \\
\text { do you consider user } \\
\text { involvement in the early } \\
\text { design stages useful? }\end{array}$ & $\begin{array}{l}\text { Yes, to ensure commitment in a } \\
\text { team }\end{array}$ & Yes & - & Yes, see other comment & Yes \\
\hline
\end{tabular}

\begin{tabular}{|c|c|c|c|c|c|}
\hline Part 3 & G2.P1 & $\mathrm{G} 2 . \mathrm{P} 2$ & G2.P3 & G2.P4 & G2.P5 \\
\hline \multicolumn{6}{|l|}{ Reflection } \\
\hline $\begin{array}{l}3.1 \text { Prior to today's } \\
\text { session, did you consider } \\
\text { user involvement in the } \\
\text { early desigm stages } \\
\text { useful? }\end{array}$ & Yes & $\begin{array}{l}\text { Yes, each user/client has his/her } \\
\text { own opinion }\end{array}$ & $\begin{array}{l}\text { Yes, it gives insights into the } \\
\text { needs and requirements of users }\end{array}$ & $\begin{array}{l}\text { Yes, the future user is the future } \\
\text { client. He/she should be } \\
\text { satisfied otherwise he/she won't } \\
\text { return }\end{array}$ & $\begin{array}{l}\text { Yes, the end-user will evaluate } \\
\text { the machine/ unit on various } \\
\text { points, such as hygiene, costs, } \\
\text { etc. }\end{array}$ \\
\hline $\begin{array}{l}3.2 \text { After today's session, } \\
\text { do you consider usere } \\
\text { involvement in the early } \\
\text { design stages useful? }\end{array}$ & Yes & $\begin{array}{l}\text { Yes, each user/client has his/her } \\
\text { own opinion }\end{array}$ & Yes & Yes, see above & $\begin{array}{l}\text { Yes, consumer insights are very } \\
\text { important }\end{array}$ \\
\hline
\end{tabular}




\section{C.2 Cross-company evaluation 3}

In the third cross-company evaluation session participants from companies $A$ and $B$ were invited to experience and evaluate the Virtual Annotation application, originally developed for company C. After experiencing the prototype (each company carried out a small test case using the application), the participants filled out the evaluation form included in appendix C.2.1. The results of this evaluation are included in appendix C.2.2

- All forms and answers have been translated from Dutch.

\section{C.2.1 CCE3 forms}

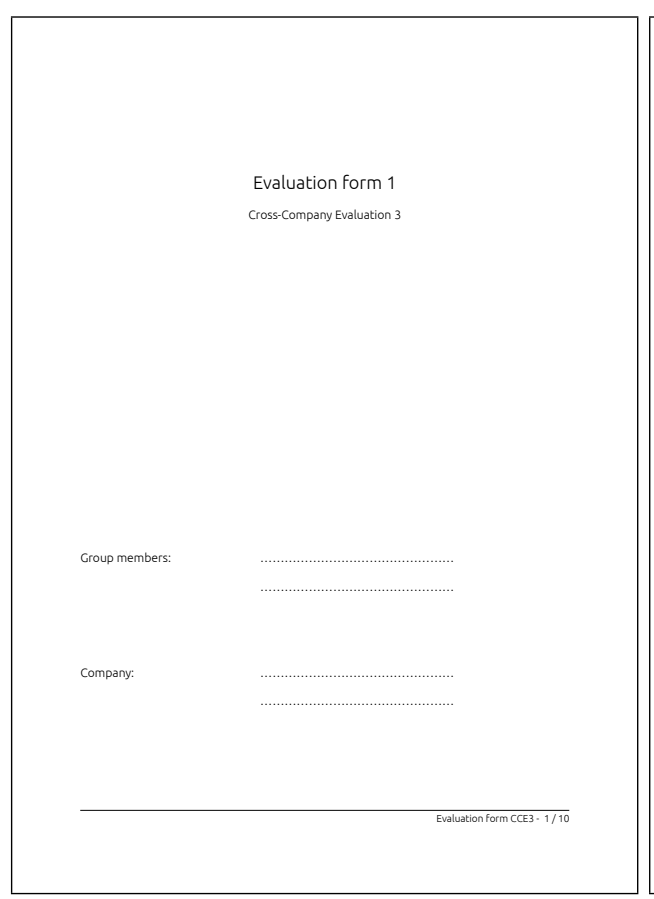

Current approach

This part of the form investigates how you would currently support a session like the one

conducted in the case study.

Situation:

You are involved in the early stage development of a new product. Until now, the You are involved in the early stage development of anew product.
product only existed 'on paper' $(i$. a. a specification document or initital sketch of functionality). The eim is to review the product toncept from a very broad d erspective,
including end-users and /or customers, in order to identify problems and issues, and to
evaluate and improve the product concept

How would you currently facilitate such a session

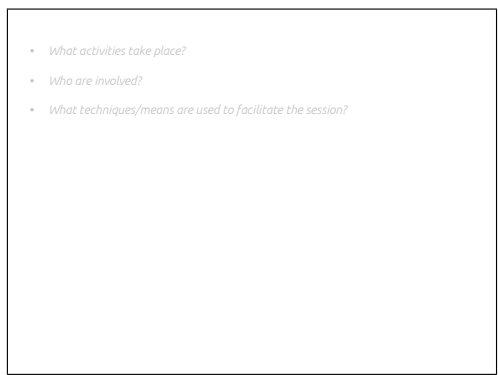




\section{CCE3 forms (continued)}

Comparison to current approach

Compare your 'current approach' (as described on the previous page) to the approad presented in the Virtual Annotation application. Try to think about the differences

Indicate the added value of the Virtual Annotation application in the form below.

\begin{tabular}{|c|c|c|c|}
\hline Added value & Do not agree & Neutral & Agree \\
\hline $\begin{array}{l}\text { Gives better insights into future use } \\
\text { situations }\end{array}$ & 0 & 0 & 0 \\
\hline 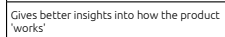 & 0 & 0 & 0 \\
\hline $\begin{array}{l}\text { It facilitates communication between } \\
\text { stakeholders }\end{array}$ & 0 & 0 & 0 \\
\hline $\begin{array}{l}\text { It helps with identifying previously } \\
\text { undiscovered issues }\end{array}$ & 0 & 0 & 0 \\
\hline $\begin{array}{l}\text { Ithelps with the generation of solutions } \\
\text { for issues }\end{array}$ & 0 & 0 & 0 \\
\hline $\begin{array}{l}\text { It helps with explaining specific issues or } \\
\text { problematic situations }\end{array}$ & 0 & 0 & $\circ$ \\
\hline It saves time & 0 & 0 & 0 \\
\hline $\begin{array}{l}\text { It facilitates collaboration within the } \\
\text { design team }\end{array}$ & 0 & 0 & 0 \\
\hline $\begin{array}{l}\text { It provides better opportunities for } \\
\text { involving external stakeholders in the } \\
\text { design activity }\end{array}$ & 0 & 0 & 0 \\
\hline
\end{tabular}

The Virtual Environment

The following questions are about the virtual environment as presented in the case study.

approach.

Functionality

The test/study would primarily be for (one answer)

o Generating new solutions

O Visualisation and presentation of solutions and problem situations

Evaluation of new solutions or product concepts

o something else:

The following table lists the core functions of the application. Please indicate whether you fider the functionality important (1=unimportant, $5=$ important), and whether this

\begin{tabular}{|c|c|c|}
\hline Functionality & Priority & $\begin{array}{l}\text { Implementation OK? If not, please suggest } \\
\text { improvements }\end{array}$ \\
\hline 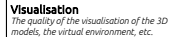 & $1 / 2 / 3 / 4 / 4 / 5 \mid$ & \\
\hline 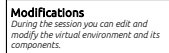 & $1 / 2 / 3 / 4 / 4 / 5$ & \\
\hline 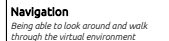 & $1 / 2 / 3 / 4 / 4 / 5$ & \\
\hline
\end{tabular}

Valuation form CCE3 $5 / 10$
Does the use of the virtual environment skew any other benefits when compared to the traditional approach? If so, please describe them.

O Yes, namely:

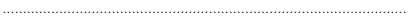

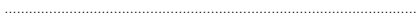

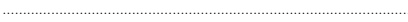

Does the use of the virtual environment skew any drawbacks when compared to the traditional approach? If so, please describe them

O Yes, namely:

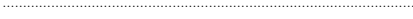

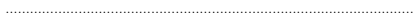

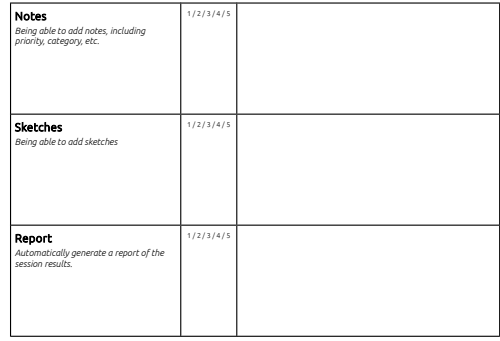

Any other suggestions that would improve the presented application?

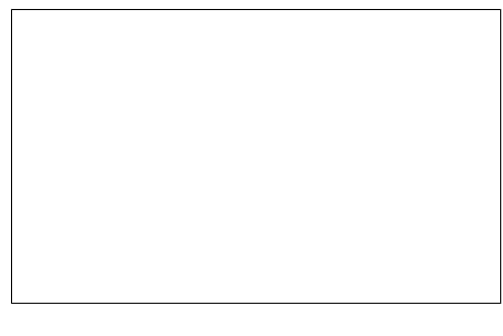




\section{CCE3 forms (continued)}

Interaction with the virtual environment

You experienced 2 forms of interaction with the virtual environment:

1. via a moderator

2. via individual input devices

What are the most relevant benefits and drawbacks of each of these forms of interaction

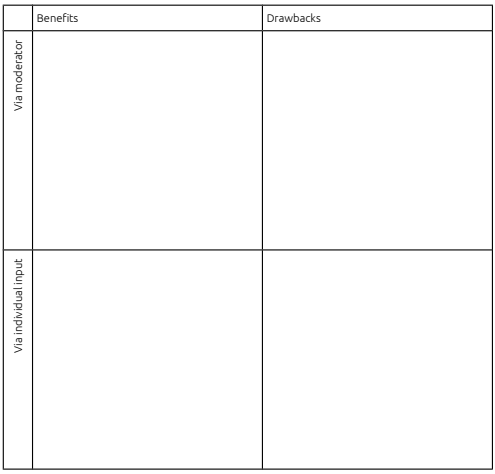

on in your company

Is this type of VR application useful for your design domain? Why (not)?

O Yes/no, because:

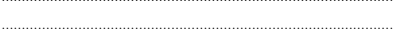

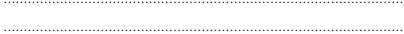

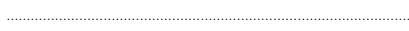

$\ldots \ldots \ldots \ldots \ldots$

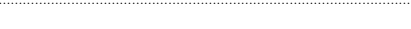

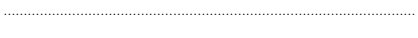

Did the form of interaction stimulate participation in the session?

\begin{tabular}{|l|c|c|}
\hline \multicolumn{1}{|c|}{} & Via moderator & Via individual input \\
\hline Not at all & 0 & 0 \\
\hline Only in the beginning & 0 & 0 \\
\hline Sometimes & 0 & 0 \\
\hline Yes & 0 & 0 \\
\hline
\end{tabular}

Did the form of interaction stimulate group collaboration?

\begin{tabular}{|l|c|c|}
\hline & Via moderator & Via individual input \\
\hline Not at all & $\circ$ & $\circ$ \\
\hline Only in the beginning & $\circ$ & 0 \\
\hline Sometimes & 0 & 0 \\
\hline Yes & 0 & 0 \\
\hline
\end{tabular}

Which form of input do you prefer, and why?

(a)

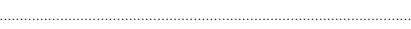

What influence does the form of input have on the session?

年

(1)

Evaluation form CCE3 $-8 / 10$

In which design phase(s) would you deploy this application?

\begin{tabular}{|l|l|}
\hline Design phase & Anticipated added value \\
\hline & \\
\hline & \\
\hline & \\
\hline & \\
\hline & \\
\hline & \\
& \\
\hline
\end{tabular}




\section{C.2.2 CCE3 results}

\begin{tabular}{|c|c|c|}
\hline Part 1 & Company A & Company B \\
\hline \multicolumn{3}{|c|}{ 1.1 How would you currently facilitate such a session? } \\
\hline & $\begin{array}{l}\text { Activities: discuss architecture, functions. } \\
\text { Conducting tests with experts, doing } \\
\text { brainstorm sessions, read test reports, conduct } \\
\text { usability evaluations } \\
\text { Who are involved: could be anyone from the } \\
\text { project, always multi-disciplinary, anyone can } \\
\text { be invited } \\
\text { Tools: modelling is done in CAD, and then } \\
\text { printed for a meeting. Depending on the stage } \\
\text { of the project: definition and problem analysis } \\
\text { takes sketches, later stages take } 1: 1 \text { models or } \\
\text { wooden mock-ups. Visualisation is key; you } \\
\text { need to be able to 'walk around' the product }\end{array}$ & $\begin{array}{l}\text { Activities: make interaction models, add } \\
\text { interactions. This involves vehicle definition, } \\
\text { vehicle control and testing. Tools depend on } \\
\text { the topic of interest. }\end{array}$ \\
\hline \multicolumn{3}{|c|}{1.2 Indicate the added value of the Virtual Annotation application in the form below. } \\
\hline $\begin{array}{l}\text { Gives better insights into } \\
\text { future use situations }\end{array}$ & Neutral & Yes \\
\hline $\begin{array}{l}\text { Gives better insights into how } \\
\text { the product 'works' }\end{array}$ & No & No \\
\hline $\begin{array}{l}\text { It facilitates communication } \\
\text { between stakeholders }\end{array}$ & Yes, because of the annotation feature & Yes \\
\hline $\begin{array}{l}\text { It helps with identifying } \\
\text { previously undiscovered issues }\end{array}$ & Yes, by including the context & Yes \\
\hline $\begin{array}{l}\text { It helps with the generation of } \\
\text { solutions for issues }\end{array}$ & Neutral & Neutral \\
\hline $\begin{array}{l}\text { It helps with explaining specific } \\
\text { issues or problematic } \\
\text { situations }\end{array}$ & $\begin{array}{l}\text { Neutral, not sure if it adds anything to current } \\
\text { way of working }\end{array}$ & Yes \\
\hline It saves time & Yes, capturing all notes quickly & Neutral \\
\hline $\begin{array}{l}\text { It facilitates collaboration } \\
\text { within the design team }\end{array}$ & $\begin{array}{l}\text { Yes, big groups take large screens and a } \\
\text { moderator }\end{array}$ & Neutral \\
\hline $\begin{array}{l}\text { It provides better } \\
\text { opportunities for involving } \\
\text { external stakeholders in the } \\
\text { design activity }\end{array}$ & $\begin{array}{l}\text { Yes, engineers understand technical drawings, } \\
\text { others don't. People 'from the outside' should } \\
\text { be involved here. }\end{array}$ & Neutral \\
\hline \multicolumn{3}{|c|}{ 1.3 Does the use of the virtual environment skew any other benefits when compared to the traditional approach? } \\
\hline & $\begin{array}{l}\text { The primary benefit is the ability to add } \\
\text { annotations, and capture all the remarks and } \\
\text { notes }\end{array}$ & - \\
\hline \multicolumn{3}{|c|}{ 1.4 Does the use of the virtual environment skew any drawbacks when compared to the traditional approach? } \\
\hline & $\begin{array}{l}\text { You have to be co-located, big/complex } \\
\text { products may become difficult to render real- } \\
\text { time, mistakes in the environment trigger } \\
\text { extra discussions }\end{array}$ & $\begin{array}{l}\text { The situation is too much simplified, causing } \\
\text { problems to be overlooked }\end{array}$ \\
\hline
\end{tabular}




\section{CCE3 results (continued)}

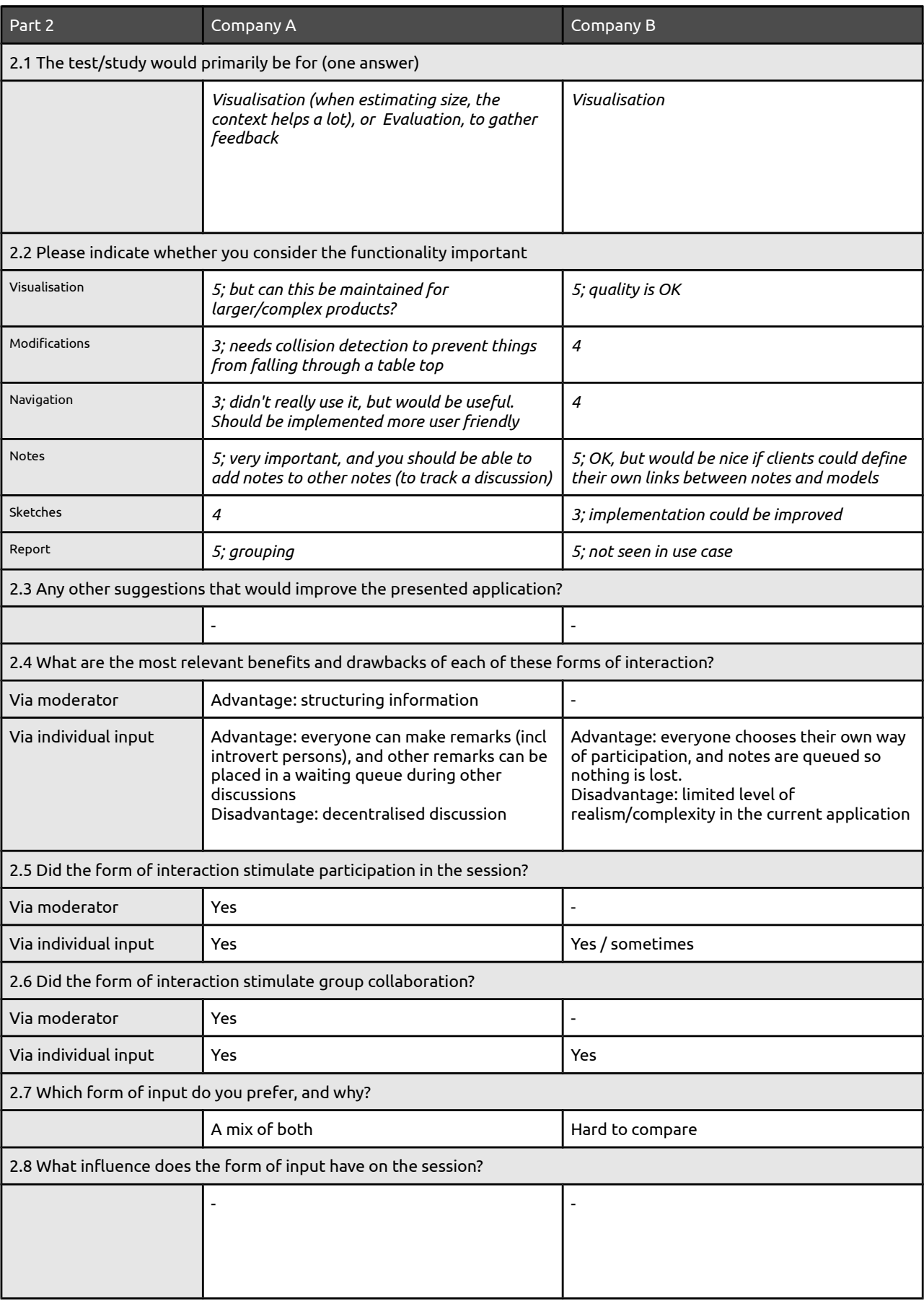




\section{CCE3 results (continued)}

\begin{tabular}{|c|c|c|}
\hline Part 3 & Company A & Company B \\
\hline \multicolumn{3}{|c|}{ 3.1 Is this type of VR application useful for your design domain? Why (not)? } \\
\hline & $\begin{array}{l}\text { Yes, for gathering feedback and for facilitating } \\
\text { multidisciplinary meetings }\end{array}$ & $\begin{array}{l}\text { Not really, our product needs to be able to } \\
\text { move around in the environment. Interaction } \\
\text { with its context is completely different }\end{array}$ \\
\hline \multicolumn{3}{|c|}{ 3.2 Who would you involve in Virtual Annotation sessions? (i.e. its use, not its development) } \\
\hline & $\begin{array}{l}\text { Depends on the session and phase in the } \\
\text { development process }\end{array}$ & $\begin{array}{l}\text { Testing department, vehicle definition, layout, } \\
\text { product planning }\end{array}$ \\
\hline \multicolumn{3}{|c|}{3.3 In which design phase(s) would you deploy this application? } \\
\hline & In any phase where prototypes are used & $\begin{array}{l}\text { - Concept phase: for the identification } \\
\text { of unexpected interactions with the } \\
\text { environment. } \\
\text { - Could also be used to visualise } 3 D \\
\text { envelopes for the engineering } \\
\text { department }\end{array}$ \\
\hline
\end{tabular}




\section{CCE3 results (continued)}

\begin{tabular}{|c|c|c|}
\hline SWOT Analysis & Company A & Company B \\
\hline \multicolumn{3}{|c|}{ Custom software } \\
\hline Strengths & Annotation \& reporting capabilities & $\begin{array}{l}\text { Able to streamline this tool/application with } \\
\text { the existing product development process }\end{array}$ \\
\hline Weaknesses & $\begin{array}{l}\text { Importing models is so complex/difficult (or } \\
\text { doesn't work with the right } \\
\text { colors/textures/etc.) that you probably won't } \\
\text { use it in the end }\end{array}$ & $\begin{array}{l}\text { Takes too much time } \\
\text { When/where do you stop with developing the } \\
\text { tool / application? }\end{array}$ \\
\hline Opportunities & $\begin{array}{l}\text { It could be developed into a 'proper' product, } \\
\text { when the modelling/import functions are OK } \\
\text { and the annotation and reporting function is } \\
\text { implemented }\end{array}$ & Theoretically everything is possible \\
\hline Threats & & $\begin{array}{l}\text { Takes a lot of time/effort to learn a new tool. } \\
\text { Implementation is difficult because } \\
\text { ideas/requirements change all the time, and } \\
\text { it's not really our job to create software (or } \\
\text { have software created for us) }\end{array}$ \\
\hline \multicolumn{3}{|l|}{ CAD extension } \\
\hline Strengths & $\begin{array}{l}\text { This sounds like the easiest way ('no problems } \\
\text { expected') }\end{array}$ & $\begin{array}{l}\text { You stay close to the orignial/known product } \\
\text { (i.e. CAD software) }\end{array}$ \\
\hline Weaknesses & $\begin{array}{l}\text { You'd probably lack a good documentation } \\
\text { function, and therefore end up using a custom } \\
\text { solution/plugin for this. We're not sure if these } \\
\text { functions (annotation/documentation) are } \\
\text { available for our CAD platform }\end{array}$ & $\begin{array}{l}\text { Not sure if this can do everything we } \\
\text { want/need }\end{array}$ \\
\hline Opportunities & $\begin{array}{l}\text { Plenty of opportunities; if this } \\
\text { extension/plugin exists it would offer a lot of } \\
\text { new solutions for us }\end{array}$ & $\begin{array}{l}\text { Relatively low costs to achieve this particular } \\
\text { application }\end{array}$ \\
\hline Threats & It doesn't exist? & $\begin{array}{l}\text { You need to make a (small) investment, and } \\
\text { still run the risk that this application does not } \\
\text { skew the results we were hoping for }\end{array}$ \\
\hline \multicolumn{3}{|c|}{ Integrated CAD suite } \\
\hline Strengths & Much easier to import $3 D$ models & $\begin{array}{l}\text { One integated set of applications, should work } \\
\text { better than individual applications. Has been } \\
\text { developed, tested and validated by external } \\
\text { parties }\end{array}$ \\
\hline Weaknesses & $\begin{array}{l}\text { It is not feasible for our company to switch to } \\
\text { a new CAD system just for this application }\end{array}$ & Might be more complex \\
\hline Opportunities & $\begin{array}{l}\text { A light version would be suffificent, we don't } \\
\text { need model generation functions, we need to } \\
\text { integrate existing models with an annotation } \\
\text { application }\end{array}$ & $\begin{array}{l}\text { You can do even more than you could do with } \\
\text { the CAD extension option }\end{array}$ \\
\hline Threats & $\begin{array}{l}\text { Too expensive, the other options could also } \\
\text { result in an 'integrated suite' }\end{array}$ & Too expensive \\
\hline
\end{tabular}

